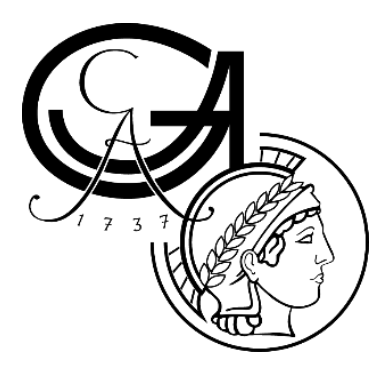

\title{
Dissecting synaptic mechanisms of sound encoding in the mouse cochlea
}

\author{
Dissertation \\ for the award of the degree \\ "Doctor of Philosophy" Ph.D. Division of Mathematics \\ and Natural Sciences \\ of the Georg August Universität Göttingen, \\ within de doctoral program: \\ Sensory and Motor Neuroscience \\ of the Georg-August University School of Science \\ (GAUSS)
}

submitted by

Philippe Jean

From Nancy, France

Göttingen, 2019 



\section{Members of the Thesis Advisory Committee:}

\section{Prof. Dr. Tobias Moser}

Molecular anatomy, physiology, and pathology of sound coding and prosthetics, Institute for Auditory Neuroscience \& InnerEarLab, University Medical Center Göttingen

\section{Prof. Dr. Thomas Dresbach}

Synaptogenesis group, Department of Anatomy and Embryology, Georg-August University, Göttingen

\section{Dr. Andreas Neef}

Biophysics of neural computation, Department of Nonlinear Dynamics and Network Dynamics group, Georg-August University, Göttingen

\section{Further members of the Examination Board:}

\section{Prof. Dr. Martin Göpfert}

Department of cellular neurobiology, Georg-August University, Göttingen

\section{Dr. Manuela Schmidt}

Somatosensory Signaling and Systems Biology Group, Max Planck Institute for Experimental Medicine, Göttingen

\section{Dr. Katrin Willig}

Optical Nanoscopy in Neuroscience, Max Planck Institute for Experimental Medicine, Göttingen

\section{Date of oral examination}




\section{Declaration}

Herewith I declare that this thesis has been written independently and with no other sources and aids than quoted.

Philippe Jean

Göttingen, January 7, 2019 


\section{List of Contents}

List of abbreviations

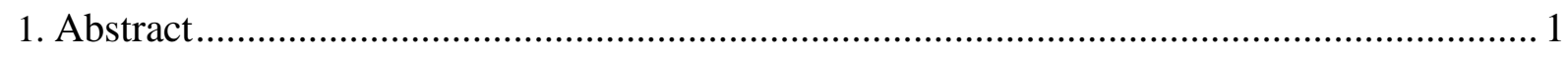

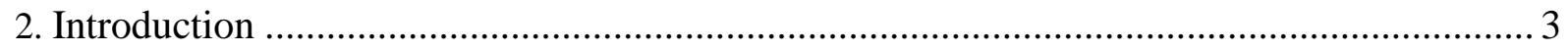

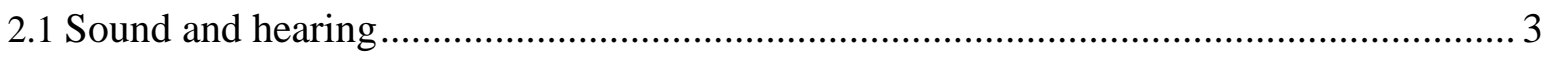

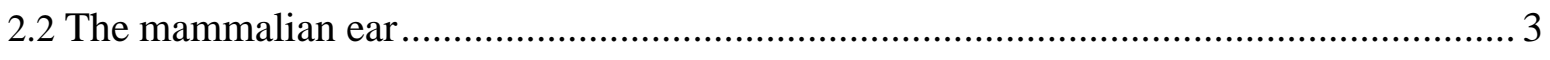

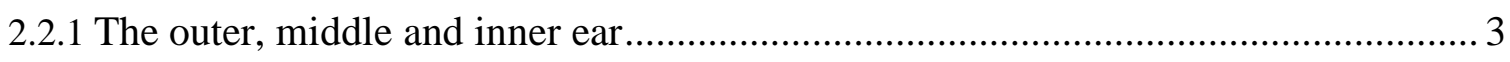

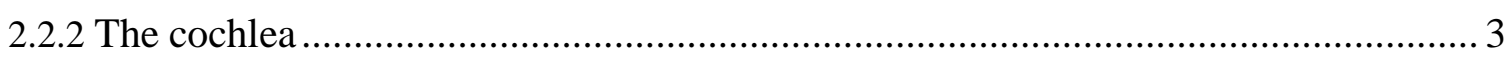

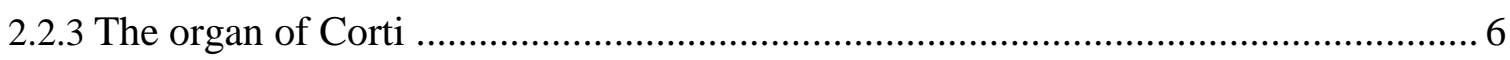

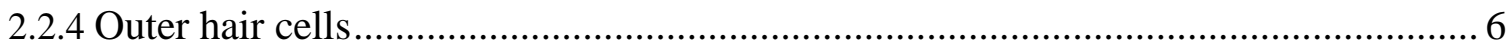

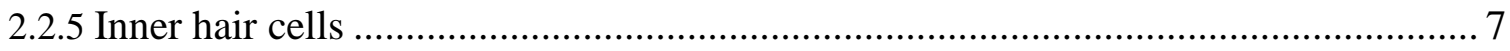

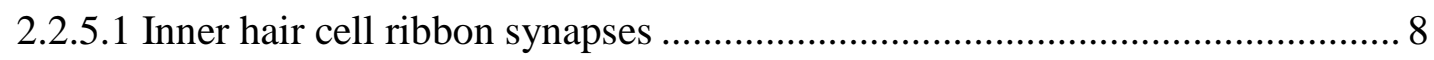

2.2.5.2 Voltage gated $\mathrm{Ca}^{2+}$ channels and their subunits...................................... 10

2.2.5.3 $\mathrm{Ca}^{2+}$ signaling at inner hair cell active zones ............................................ 15

2.2.6 Encoding of sound intensity in spiral ganglion neurons........................................ 19

2.2.7 Spatial heterogeneity of synaptic $\mathrm{Ca}^{2+}$ influx properties at inner hair cells............ 21

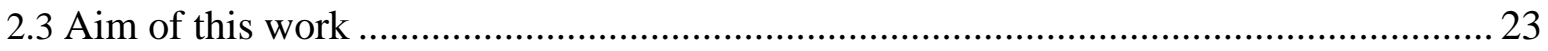

3. Chapter 1 "The synaptic ribbon is critical for sound encoding at high rates and with temporal precision"

4. Chapter 2 "Pou4f1 defines a subgroup of type I spiral ganglion neurons and is necessary for

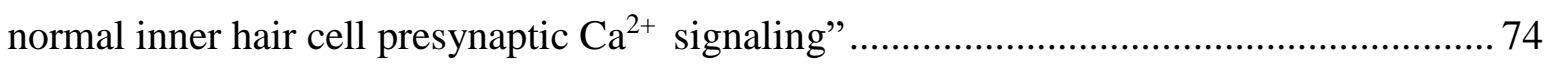

5. Chapter 3 "Intrinsic planar polarity mechanisms influence the position-dependent regulation

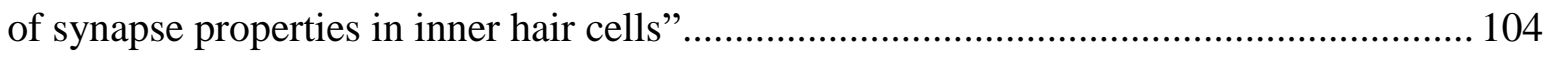

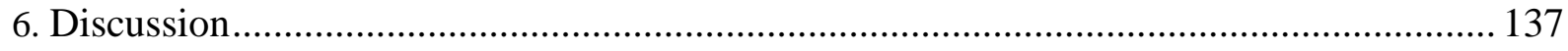

6.1 Cellular mechanisms of wide dynamic range of sound encoding ............................... 138

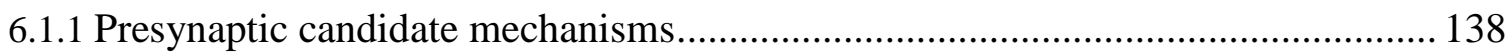

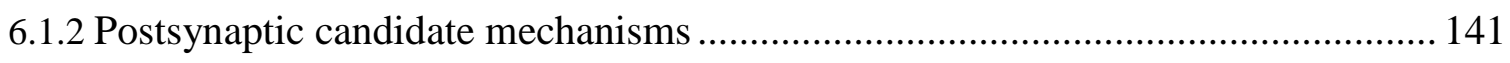

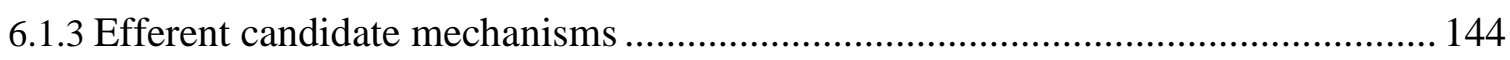

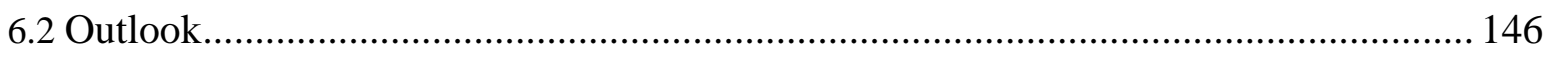

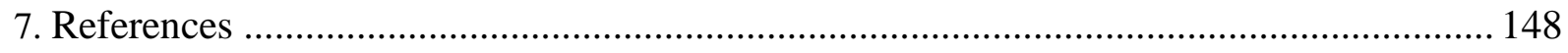

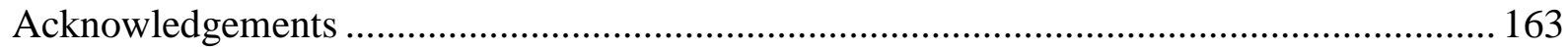




\section{List of abbreviations}

ABR

$\mathrm{ACh}$

AID

AMPA

AVCN

AZ

BAPTA

$\mathrm{CaBP}$

$\mathrm{CaM}$

CDI

$\mathrm{C}_{\mathrm{f}}$

C.M.

$\Delta \mathrm{C}_{\mathrm{m}}$

CtBP2

DPOAE

EGTA

EM

EPSC

EPSP

$\mathrm{F}_{0}$

$\Delta \mathrm{F}$

$\Delta \mathrm{F}_{\max }$

FS

FV

FWHM

GPCR

$\mathrm{HC}$

HPF

IHC

IV

$\mathrm{k}$

$\mathrm{KO}$

L.A.

LJP auditory brainstem responses

acetylcholine

$\alpha$ interacting domain

$\alpha$-amino-3-hydroxy-5-methyl-4-isoxazolepropionic acid

anteroventral cochlear nucleus

active zone

1,2- bis (2-aminophenoxy) ethane- $N, N, N 9, N 9$-tetraacetate

calcium binding protein

calmodulin

calcium-dependent activation

characteristic frequency

center of mass

membrane capacitance changes

C-terminal binding protein 2

distortion product otoacoustic emissions

ethylene gly- col-bis-(2-aminoethyl)-N,N,N',N'-tetraacetic acid

electron microscopy

excitatory post-synaptic current

excitatory post-synaptic potential

fluorescence at resting state

fluorescence change

maximal fluorescence change

freeze substitution

fluorescence-voltage relationship

full width half maximum

$\mathrm{G}$ protein coupled receptor

hair cell

high pressure freezing

inner hair cell

current-voltage relationship

slope

knockout

long axis

liquid junction potential 
MET

MVR

MP

NPMC

$\mathrm{OHC}$

PCP

PD

PSD

PTXa

$\mathrm{Q}_{\mathrm{Ca}}$

RA

RIM

RIM-BP

RRP

$\mathrm{R}_{\text {series }}$

S.A.

S.D.

S.E.M.

SGN

SNARE

SR

STED

SV

TAMRA

UVR

VDI

VGCC

Vglut

$\mathrm{V}_{\mathrm{h}}$ mechanoelectrical transduction

multivesicular release

membrane proximal

non parametric multiple comparison

outer hair cell

planar cell polarity

presynaptic density

postsynaptic density

pertussis toxin catalytic subunit

calcium charge

ribbon associated

rab3-interacting molecule

RIM binding protein

readily releasable pool

series resistance

short axis

standard deviation

standard error of the mean

spiral ganglion neuron

soluble N-ethylmaleimide-sensitive factor attachment protein receptors

spontaneous rate

stimulated emission depletion

synaptic vesicle

carboxytetramethylrhodamine

univesicular release

voltage-dependent inactivation

voltage-gated $\mathrm{Ca}^{2+}$ channel

vesicular glutamate transporter

voltage for half activation 



\section{Abstract}

Cochlear inner hair cells (IHCs) are the genuine sensory receptors that translate sound-borne cochlear vibrations into neuronal signals via ribbon synapses with the spiral ganglion neurons (SGNs). The precise mechanisms of these ribbon synapses, the first relay of the auditory pathway, are still not fully resolved. Sound intensity coding over a wide dynamic range is thought to be fractionated through the SGNs presenting distinct firing characteristics. One hypothesis is that much of this diversity reflects the presynaptic heterogeneity observed among the active zones (AZs) of IHCs. There, a single AZ is the sole input to its associated SGN and it has been demonstrated that the AZs present very distinct properties according to their position in the IHCs. The AZs facing the spiral limbus (modiolar side) show bigger ribbons correlated with a stronger $\mathrm{Ca}^{2+}$ influx activated at more depolarized membrane potentials than those at the side facing the pillar cells (pillar side). Differences in the voltage-dependent activation of the $\mathrm{Ca}^{2+}$ channels are an attractive explanation of the diverse postsynaptic spontaneous rates, where the SGNs associated with modiolar AZs with more depolarized activation range present a low spontaneous rate, while the pillar side targeting SGNs present a higher spontaneous rate. In this thesis, I first focused on deciphering the role of the synaptic ribbon at the first auditory synapse. Together with collaborators, we characterized the morphology and physiology of the ribbonless synapses by immunofluorescence and electron microscopy as well as patch-clamp/ $\mathrm{Ca}^{2+}$ imaging of IHCs and systems physiology. We demonstrated a compensatory reorganization of the presynapses into several small ribbonless AZs, indicated a regulation of presynaptic $\mathrm{Ca}^{2+}$ influx by the ribbon and revealed a corresponding threshold increase as well as an impaired vesicle replenishment in the absence of the synaptic ribbon. The second part of my thesis aimed to decrypt the mechanisms setting the position-dependent heterogeneous properties of IHC AZs, putatively contributing to the wide dynamic range of sound encoding. Performing immunostainings and patch-clamp recordings combined with fast live $\mathrm{Ca}^{2+}$ imaging, we tested two different candidate mechanisms. We firstly investigated if the transcription factor Pou4f1, expressed nearly entirely in a type I SGN subpopulation targeting the IHC modiolar face. We suggested that Pou4f1 defines a subset of low spontaneous rate, high threshold SGNs by decreasing the presynaptic voltage sensitivity leading to a depolarized shift of $\mathrm{Ca}^{2+}$ influx activation of their associated modiolar AZs. Then, we focused on the planar polarity mechanisms dictating hair bundle orientation and apical surface asymmetry, and proposed a role for the Gai/LGN complex in regulating the position-dependent $\mathrm{AZ}$ properties in IHCs. 


\section{Introduction}

\subsection{Sound and hearing}

Sound is the principal mean of communication among animals but is also essential for their survival by indicating the location of prey or predators and by supporting navigation. Sound refers to a periodic, elastic compression and rarefaction of the transmitting medium (gas, liquid or solid). Sound hence propagates in the form of longitudinal waves (but spherically from the source) that carry two main properties, their amplitudes and frequencies. A third property, the localization can be detected thanks to a pair of ears, evaluating differences in the arrival time (low frequency sounds) and intensity (high frequency sounds) of the sound stimulus between these both ears, called interaural time and level differences. Depending on the distance between these two ears, the wave propagation angle and the constant interaural axis, time differences of a few $\mu$ s need to be detected to decipher sound origin. This requires an incredible fidelity in the transmission of sound-evoked neural signaling in the nervous systems. Moreover, the ear can process acoustic stimuli that widely range in frequency and amplitude, and mammals can encode sound pressures ranging over six orders of magnitude. The fundamental mechanisms responsible for the precise, dynamic and indefatigable sound encoding are far from being completely understood.

\subsection{The mammalian ear}

\subsubsection{The outer, middle and inner ear}

The mammalian ear is divided into the outer, middle and inner ear. The outer ear is composed of a first part, the auricle focusing and filtering the incoming sound to the second part, the ear canal. At the end of this canal, the sound waves vibrate the tympanic membrane transferring the sound into the middle ear, a cavity filled with air. The sound wave is then carried by three ossicles (malleus, incus, and stapes) to the oval window of the cochlea in the inner ear, that also houses the vestibular labyrinth that senses head position and movement (Figure 1A).

\subsubsection{The cochlea}

The cochlea is a snail shaped structure consisting of a bony core around which several turns (which number depends on the species) of three fluid-filled compartments are coiled up (Figure 
1A). These compartments are the scalae vestibuli and tympani filled with perilymph (containing a comparably low amount of $\mathrm{K}^{+}(\sim 5 \mathrm{mM})$, and the scala media, filled with endolymph that has a much higher concentration of $\mathrm{K}^{+}(\sim 160 \mathrm{mM})$ due to secretion of $\mathrm{K}^{+}$from the stria vascularis, lining the lateral wall and forming the outside boundary of the scala media (Figure 1B). When the stapes strikes the oval window, an amplification of the mechanical stimulus is made by both the larger surface of tympanic membrane as compared to the oval window membrane, and the leverage effects of the ossicles, which is essential for not reflecting the sound at the scala vestibuli fluid which has much greater impedance than air. Since this fluid is incompressible, the increase in pressure in the upper compartment, the scala vestibuli, is transmitted towards the lower compartment, the scala tympani resulting in a vibration of the basilar membrane and finally an outward bulging of the round window membrane. Both scalae are connected at the helicotrema at the apex of the cochlea. This system of pressure transfer causes a vertical travelling wave on the basilar membrane along the length of the cochlear turns (Figure 1C).

The basilar membrane is narrow and rigid at the base and wide and soft at the apex of the cochlea. These anatomical characteristics determine the inertia and stiffness of different parts of the cochlea, whereby defining their impedance. In consequence, high frequencies result in maximal movement of the basilar membrane in the basal cochlea, whereas low frequencies best vibrate the apical basilar membrane. This leads to a tonotopic frequency mapping along the length of the cochlea, covering the entire hearing range of the organism (Figure 1C). Cochlear amplification for soft sounds and nonlinear compression for strong sounds are thought to enable the auditory system to grade the output of the cochlea for changes in input despite the limited dynamic range of sensory and neural mechanisms. Knocking out $\alpha$-tectorin, detaching the tectorial membrane, generate a linear and monotonic compression of the basilar membrane. Therefore, the tectorial membrane was proposed to ensure proper outer hair cell (OHC) response (see below) to basilar membrane motion via their anchorage, required for the compression (Legan et al., 2000). The receptor organ for hearing, called organ of Corti, is sandwiched between the tectorial and basilar membranes (Figure 1D). 

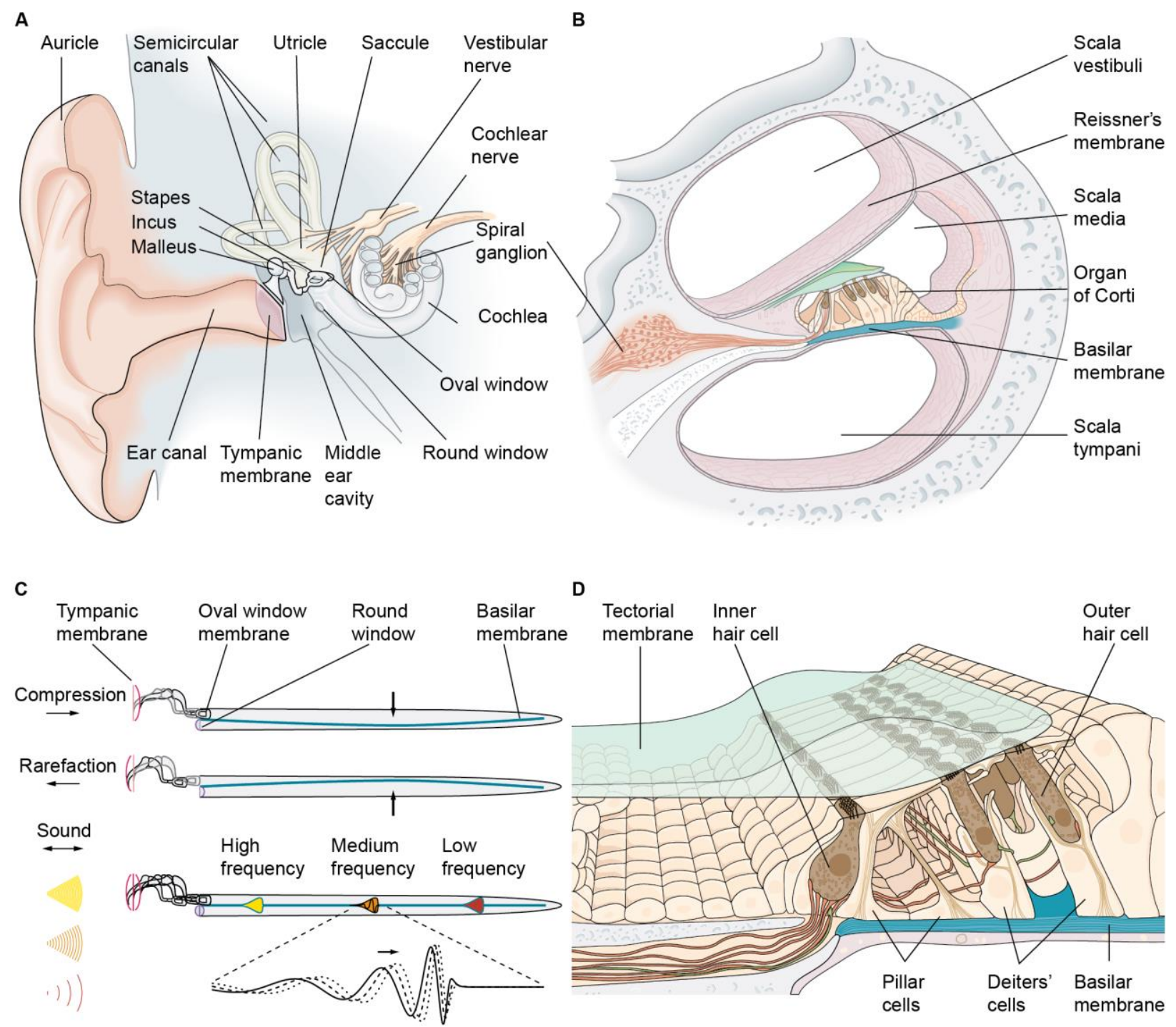

\section{Figure 1: Outer, middle and inner ear}

(A) Schematic representing the external ear that focuses and filters the incoming sound to the ear canal. At the end of this canal, the waves conduct vibrations onto the tympanic membrane transferring the sound into the middle ear cavity. The sound wave is then carried by three ossicles to the oval window of the inner ear, consisting of two parts: the cochlea and the vestibular system. (B) Schematized cross section of a cochlea showing the arrangement of 3 coiled up fluid filled compartment that are the scala vestibuli, tympani and media. In this last compartment resides the organ of Corti surmounting the basilar membrane. (C) Simplified representation of an uncoiled cochlea and its basilar membrane. The sound causes a travelling wave of the basilar membrane. Each frequency of stimulation maximally excites a particular position of the basilar membrane. (D) Schematic of the organ of Corti, consisting of one row of IHCs, three rows of OHCs and several types of supporting cells, altogether sandwiched between the tectorial and basilar membranes. Modified from (Kandel, 2012).

\subsubsection{The organ of Corti}

The organ of Corti is a highly organized structure in the cochlea responsible for the processing of the sound. Situated on top of the basilar membrane, it consists of one row of IHCs, three 
rows of OHCs and several types of supporting cells such as Hensen's cells, Deiter's cells, phalangeal cells, inner and outer pillar cells. While the hair cells (HCs) are responsible for the sensory processing, the supporting cells play important roles in homeostasis and structural support of the organ of Corti. The HCs are sandwiched between the basilar and tectorial membranes (Figure 1D). The cuticular plates of the HCs possess groups of stereocilia (so-called hair bundles) made of a dense-filament core, arranged in two to three rows and are anchored in the tectorial membrane. The stereocilia are inter-connected at their apical tips by tip links (Pickles et al., 1984), filamentous structures mainly composed mainly of cadherin 23 (Siemens et al., 2004; Söllner et al., 2004) and protocadherin 15 (for review, see Müller, 2008). They are thought to be directly linked to the mechanoelectrical transduction (MET) channels (Assad et al., 1991; Howard and Hudspeth, 1988) situated at the top of the stereocilia (Beurg et al., 2009; Jaramillo and Hudspeth, 1991; Lumpkin and Hudspeth, 1995). During sound-borne vibrations of the basilar membrane, the vertical displacement of the organ of Corti leads to a shearing movement, causing the deflection of the stereocilia, which in turn gates the MET channels. The tip links between stereocilia were suggested to work as springs ruled by Hook's law. A displacement towards the longest stereocilia causes increased tension in the tip links, which in turn opens the MET channels. The increase or decrease of cation influx from the endolymph into the cells, mainly $\mathrm{K}^{+}$, through these channels depolarizes or hyperpolarizes the cell, respectively (Corey and Hudspeth, 1979).

\subsubsection{Outer hair cells}

The active membrane electromotility of the cylindrical OHCs (and potentially active bundle movements) mechanically amplify the vibration of the cochlear partition for soft sounds. The lateral membrane of the OHCs is densely packed with prestin, an integral membrane protein which belongs to the SLC26 family of ion transporters (Lohi et al., 2000; Oliver et al., 2001; Zheng et al., 2000). This protein exhibits conformational changes upon voltage variations, resulting in stretching or shrinking of the cell, which are convened by interaction with chloride and bicarbonate anions in the cytoplasm (Oliver et al., 2001). Prestin-driven electromotility and, potentially, active bundle movements greatly amplify the vibration of the basilar membrane at a given tonotopic position for soft sounds with high frequency selectivity (Chan and Hudspeth, 2005b; Kennedy et al., 2005; Liberman et al., 2002; Zheng et al., 2000; and for review see Hudspeth, 2008). 


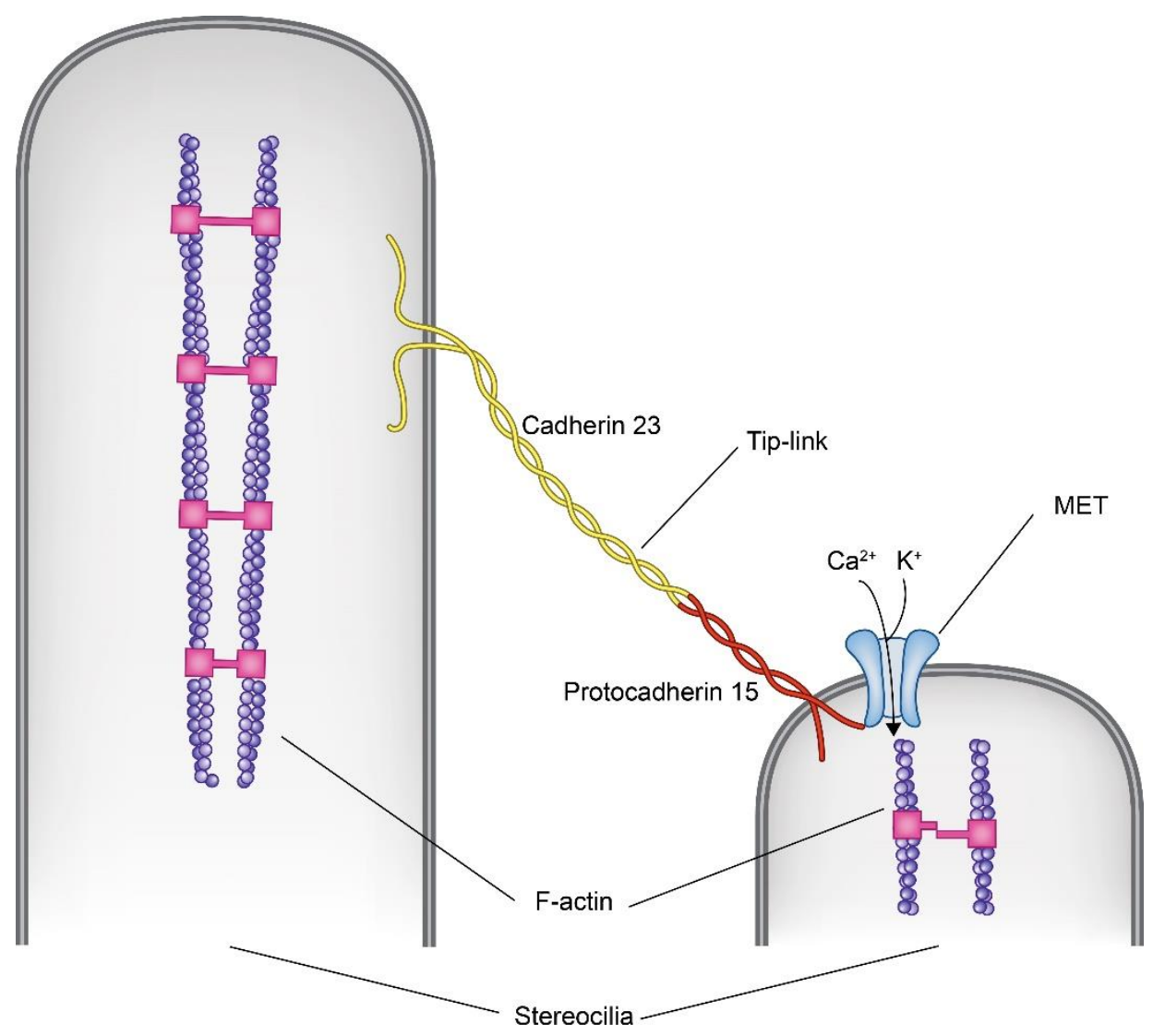

Figure 2: Stereocilia and tip links

The stereocilia are made of dense actin filaments and are inter-connected by tip links, mostly composed of cadherin 23 and protocadherin 15 . They are thought to directly connect to the MET channels. During sound detection, the vertical displacement of the basilar membrane leads to the deflection of the stereocilia, which in turn gate the MET channels. A displacement towards the longest stereocilia causes increased tension in the tip links, which in turn opens the MET channels. The increase or decrease of cation influx from the endolymph into the cells through these channels depolarizes or hyperpolarizes the cell, respectively. Not to scale. Modified from (Lukacs, 2016).

\subsubsection{Inner hair cells}

Similarly to the OHCs, there are hair bundles arranged in two to three rows of stereocilia on the cuticular plate of the IHCs as well (for review, see Raphael and Altschuler, 2003). Each IHC possesses 20-50 stereocilia (depending on species and location along the basilar membrane), each presenting a diameter of $\sim 250 \mathrm{~nm}$ and being inter-connected via their tip links. During sound-borne vibrations of the basilar membrane, while the hair bundles of the OHCs are deflected, the resulting movement of fluid radially displaces the hair bundles of the IHCs, permitting influx of cations. As for OHCs, this leads to a quick depolarization of the IHC membrane potential, and a shift towards the smallest stereocilia row causes a slackening of the 
tip links, resulting in closing of MET channels and a more negative membrane potential. The depolarization activates voltage-gated calcium $\left(\mathrm{Ca}^{2+}\right)$ channels (VGCCs) at the ribbon synapses, ultimately leading to an entry of $\mathrm{Ca}^{2+}$ ions inside the cell, thereby triggering exocytosis of glutamate into the synaptic cleft. However, the precise synaptic transmission machinery is still enigmatic due to the peculiar features making the IHC ribbon synapse unique (Figure 3).

\subsubsection{Inner hair cell ribbon synapses}

The ribbon synapses formed by IHCs and postsynaptic SGNs are the first relay of the auditory pathway. IHCs must be able to detect sudden sound pressure changes in the environment and to convey the signal faithfully to the downstream neurons. To support the incessant stimulation of these receptors by the environment, the synapses have to maintain high rates of sustained release. Therefore, vesicle recycling has to be rapid, efficient and indefatigable. The hallmark feature to which ribbon synapses owe their name is a proteinaceous structure called the synaptic ribbon, which can be found at the synapses of auditory and vestibular hair cells, as well as photoreceptors, retinal bipolar cells, and pinealocytes (for review, see Fuchs et al., 2003; Lenzi and von Gersdorff, 2001; Sterling and Matthews, 2005).

At mouse IHC synapses, the ribbons are identified as spherical, ellipsoidal, or barshaped electron-dense body at the electron microscopy (EM) level. The size of these bodies varies among and within cells (spanning from 100 to $500 \mathrm{~nm}$ in the mouse IHCs). By molecules of unknown identity, a large number of synaptic vesicles (SVs, increasing with the size of the ribbon) is tethered to the ribbon structure forming a SV-halo around the ribbon body (Figure 3). The main and core-component of the ribbon is RIBEYE (Schmitz et al., 2000), a protein unique to ribbon synapses. Large agglomerates of RIBEYE compose around two-third of the ribbon (for review, see Zanazzi and Matthews, 2009). RIBEYE has an N-terminal A domain and a Cterminal B domain. The A domain is thought to have a structural role, whereas the B domain is probably exposed in the cytosol, and is therefore suggested to have a metabolic function. Interestingly, the $\mathrm{B}$ domain is identical to the $\mathrm{C}$-terminal binding protein 2 (CtBP2), a transcription factor ubiquitously found in most tissues, making the specific deletion of the ribbon difficult. Despite decades of work, the role of the synaptic ribbon in sensory cells is still not completely resolved. The role of the ribbon has been widely investigated including approaches that employed natural modification of ribbon size or abundance (Hull et al., 2006; 
Mehta et al., 2013), photoablation (Mehta et al., 2013) and genetic manipulation removing or altering the ribbons (Frank et al., 2010; Khimich et al., 2005; Lv et al., 2016; Sheets et al., 2017). Lately, specific knock out of the Ribeye A domain achieved complete lack of ribbons from the mouse retina (Maxeiner et al., 2016) and organ of Corti (Becker et al., 2018; Jean et al., 2018). A long-standing hypothesis poses that the ribbon operates as a conveyor belt replenishing release sites by facilitated diffusion of SVs on the ribbon surface towards the site of consumption and promotes SV priming (Becker et al., 2018; Frank et al., 2010; Graydon et al., 2014; Jean et al., 2018; Mehta et al., 2013; Zenisek et al., 2000, for a deviating view see Jackman et al., 2009, where the ribbon is a safety belt slowing down the exocytosis in cones). The ribbon has also been proposed to establish a large complement of vesicular release sites and $\mathrm{Ca}^{2+}$ channels at the $\mathrm{AZ}$ (Frank et al., 2010), to ensure close spatial coupling of $\mathrm{Ca}^{2+}$ channels and vesicular release sites (Maxeiner et al., 2016), or enhancing presynaptic $\mathrm{Ca}^{2+}$ signals by limiting diffusional $\mathrm{Ca}^{2+}$ spread (Graydon et al., 2014). Finally, the ribbon has also been proposed to contribute to multivesicular release (MVR) by coordinated or homotypic fusion potentially explaining the strong heterogeneity in size and shape of the spontaneous excitatory postsynaptic currents (EPSCs). However, this mechanism has been questioned recently, and univesicular release (UVR) has been proposed instead, where a single vesicle could either engage a full collapse or a pore flickering fusion (Chapochnikov et al., 2014; Grabner and Moser, 2018; Huang and Moser, 2018).

Several other scaffold proteins have also been identified in ribbon synapses. CAST and ELKS were shown to be indispensable for synapse development, function and maintenance in the mouse retina (tom Dieck et al., 2012; Hagiwara et al., 2018). The selective deletion of long Piccolo isoforms left the retinal ribbon synapses unchanged (Regus-Leidig et al., 2013), while the knock-down of Piccolino, a short ribbon-specific Piccolo variant, disrupted the presynaptic ribbon plate-shaped morphology (Regus-Leidig et al., 2014). The ribbon anchoring role of Bassoon has been put in evidence by the reduced number of anchored ribbons and the presence of cytosolic floating ribbons observed in the mutant mouse (Dick et al., 2003; Frank et al., 2010; Khimich et al., 2005). Moreover, super resolution stimulated emission depletion microscopy (STED) described the misalignment of $\mathrm{Ca}^{2+}$ channel clusters, even in the ribbon-anchored synapses of Bassoon mutant mice. Finally, membrane capacitance $\left(\mathrm{C}_{\mathrm{m}}\right)$ measurements exposed a reduction of the fast component of exocytosis, reflecting the readily releasable pool (RRP). All together, these synaptic alterations resulted in an impaired auditory function (Buran et al., 2010; Jing et al., 2013). Whether the reduction of the exocytosis is due to the loss of Bassoon or to the loss of ribbon still needs to be clarified. No major alteration of the exocytosis at IHCs 
has been described by $\mathrm{C}_{\mathrm{m}}$ measurements in IHCs of Ribeye knockout (KO) mice where the synaptic ribbons were completely removed, likely due to compensation involving the formation of several small ribbonless AZs in IHCs (Becker et al., 2018; Jean et al., 2018). Nonetheless, analysis of sound encoding in vivo revealed altered SV release and refilling phenotype at high rates of stimulation. Interestingly, IHC synapses appear to operate independently of the classical neuronal soluble N-ethylmaleimide-sensitive factor attachment protein receptors (SNAREs) SNAP-25, syntaxin-1, and synaptobrevin-1 or synaptobrevin-2 (Nouvian et al., 2011). Moreover, unlike glutamatergic neurons mainly using vesicular glutamate transporter isoforms Vglut1 or Vglut2, IHCs express Vglut3 (Ruel et al., 2008; Seal et al., 2008, also found in glial cells and non-glutamatergic neurons). Furthermore, synaptophysin, a synaptic vesicle marker, as well as the $\mathrm{Ca}^{2+}$ sensors synaptotagmins I and II were not detected in the mature IHCs (Reisinger et al., 2011; Safieddine and Wenthold, 1999), for controversy see (Beurg et al., 2010; Johnson et al., 2010). The VGCCs have also been shown to be localized at the AZ below the ribbon (Brandt et al., 2005), however the detailed mechanisms by which all these protagonists interact with each other is still poorly resolved. Most importantly, the subunit composition of the VGCC clusters is of great importance to shape the $\mathrm{Ca}^{2+}$ signal at this atypical synapse.

\subsubsection{Voltage gated $\mathrm{Ca}^{2+}$ channels and their subunits}

$\mathrm{Ca}^{2+}$ signal plays many central roles in cells. At conventional synapses the neurotransmitter release is principally caused by $\mathrm{Ca}^{2+}$ influx through P/Q-, $\mathrm{N}-$, and/or R-type VGCCs. Unlike those conventional synapses, the ribbon synapses in IHCs are not governed by action potentials, but driven by a graded potential and are therefore equipped with specific mechanisms regulating the VGCCs to faithfully transduce the signal into neurotransmission. Remarkably, the predominant $\mathrm{Ca}^{2+}$ channel isoform found in IHCs is the L-type Cav1.3 (90\% of the $\mathrm{Ca}^{2+}$ channels, while the rest possibly comprise different kind of L-type (Cav1.4), as well as R-type (Cav2.3) channels (Brandt et al., 2003). Hence, IHCs exhibit dihydropyridinesensitive $\mathrm{Ca}^{2+}$ currents (a class of drugs which can have either inhibitory (Isradipine, Nifedipine) or augmenting effects (BayK8644) on the channel opening probability) that have fast activation kinetics, open at comparably negative potentials (between -60 and $-50 \mathrm{mV}$ ), and exhibit very little inactivation (Cui et al., 2007), rendering them suitable to support sustained exocytosis (Brandt et al., 2003; Dou et al., 2004; Platzer et al., 2000). Cav1.3 Ca ${ }^{2+}$ channels are clustered at the ribbon synapses (Brandt et al., 2005; Roberts et al., 1990; Zenisek 
et al., 2003), leaving a relatively low number of extrasynaptic $\mathrm{Ca}^{2+}$ channels (Brandt et al., 2005; Neef et al., 2018). Consequently, mice lacking the Cav1.3 $\mathrm{Ca}^{2+}$ channels are congenitally deaf (Platzer et al., 2000), due to failure to trigger exocytosis (Brandt et al., 2003).

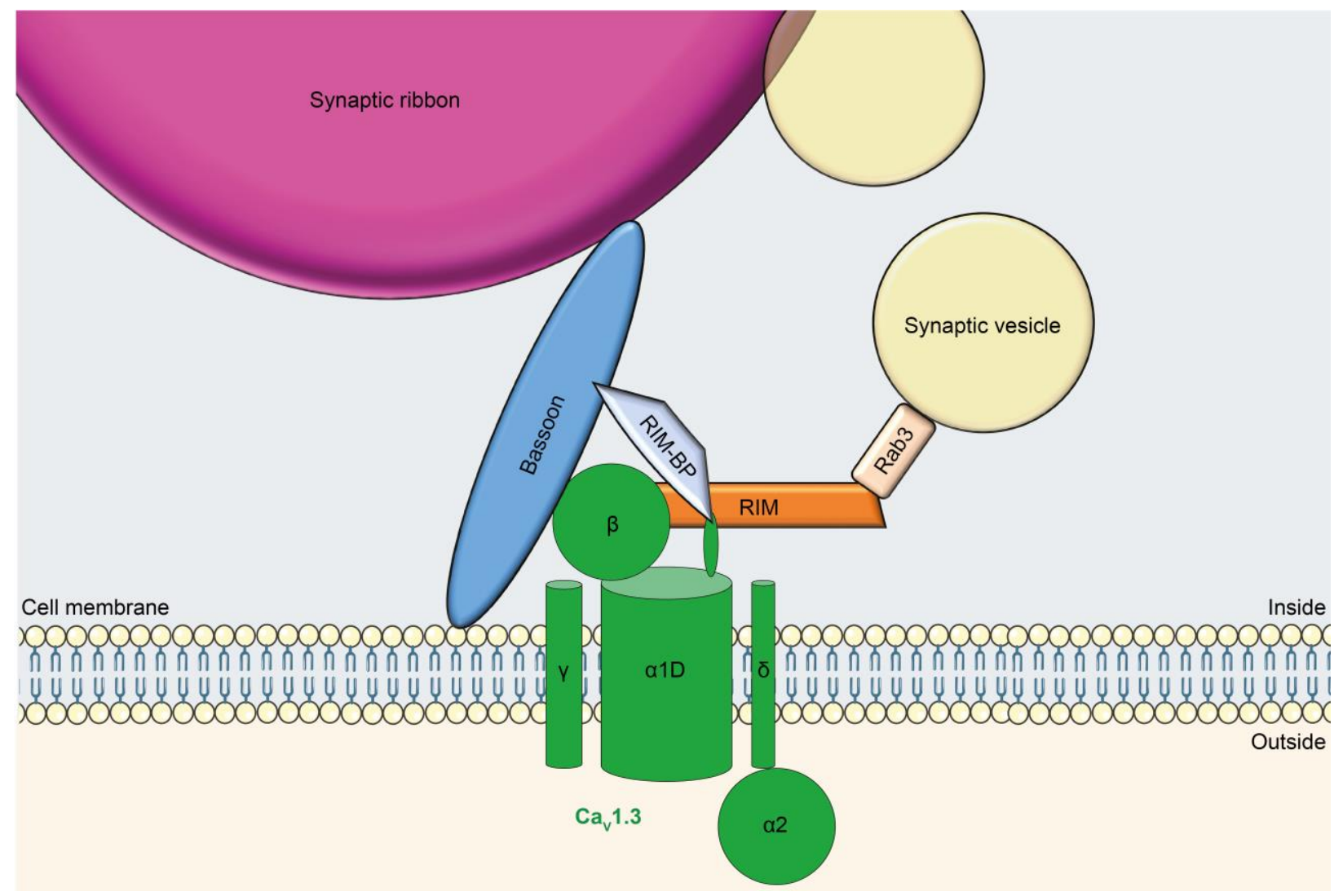

\section{Figure 3: Inner hair cell active zone}

Illustration of the synaptic ribbon (magenta), selected cytomatrix AZ proteins, and $\mathrm{Ca}_{v} 1.3 \mathrm{Ca}^{2+}$ channels that group at the base of the ribbon. The scaffolding proteins Bassoon (the anchor of the ribbon) and RIM are involved in clustering the synaptic $\mathrm{Ca}^{2+}$ channels through direct or indirect interactions. RIM binding proteins (RIM-BPs) interact with both RIM and Bassoon and are molecular linker candidate between $\mathrm{Ca}^{2+}$ channels and vesicular release sites. RIM further interacts with SVs through Rab3 interaction. Not to scale.

Based on fluctuation analysis of $\mathrm{Ca}^{2+}$ currents, it has been proposed that mature mouse IHCs at the apex possess on average about 1,700 VGCCs (Brandt et al., 2005). Immature IHCs are thought to contain more $\mathrm{Ca}^{2+}$ channels (Beutner and Moser, 2001; Brandt et al., 2003; Johnson et al., 2005; Wong et al., 2014; Zampini et al., 2010). Assuming an extra-synaptic density of 1 channel $/ \mu \mathrm{m}^{2}$, the average number of Cav1.3 channels per AZ was thus estimated to be $\approx 80$ in mature apical IHCs (Brandt et al. 2005). In a later study, using two different $\mathrm{Ca}^{2+}$ imaging approaches via microiontophoresis and non-stationary fluctuation analysis, the number of $\mathrm{Ca}^{2+}$ channels per AZ was spanning from 30 to 330 (mean of 125) and 20 to 300 (mean of 78), respectively (Neef et al., 2018). The VGCCs consist of one main pore-forming 
$\alpha 1$ subunit, assembling with $\beta, \gamma, \alpha_{2}$ and $\delta$ auxiliary subunits that shape the trafficking and functional properties of the channel (Figure 4) (Ertel et al., 2000), and can be arranged into five different types depending on their current properties and drug sensitivity (for review, see Tsien et al., 1995). L-type $\mathrm{Ca}^{2+}$ channels exhibit long-lasting and large currents. They are highvoltage activated and can be blocked by dihydropyridines, phenylalkylamines and benzothiazepines. P/Q- and N- type channels also activate at high voltages but have a lower conductance than L-type. P/Q-type are sensitive to w-Aga IVA toxin (Llinás et al., 1989), while N-type channels are sensitive to w-conotoxin GVIA (Nowycky et al., 1985). R-type channels activate at high voltages as well (Niidome et al., 1992) but are resistant to toxins (Williams et al., 1994), and T-type channels are a class of low-voltage activated channels presenting only small and rapidly inactivating currents (Carbone and Lux, 1984; Nilius et al., 1985).

The Cav $\alpha_{1}$ subunits are large multi transmembrane domains transcribed from ten different genes. Each of their four homologous domains are linked to each other by cytoplasmic loops. The S4 segment of each domain contains multiple positively charged residues and is thought to act as voltage sensor (Glauner et al., 1999; Logothetis et al., 1992), whereas the S5 and S6 segments form the pore region. Furthermore, interaction sites with other $\mathrm{Ca}^{2+}$ channel subunits and molecules are present. Multiple C-terminal splice variants of Cav1.3 $\mathrm{Ca}^{2+}$ channels were identified, translated to long and short Cav1.3 isoforms, exhibiting different activation, inactivation and opening probability (Bock et al., 2011; Shen et al., 2006; Singh et al., 2008; Tan et al., 2011). 


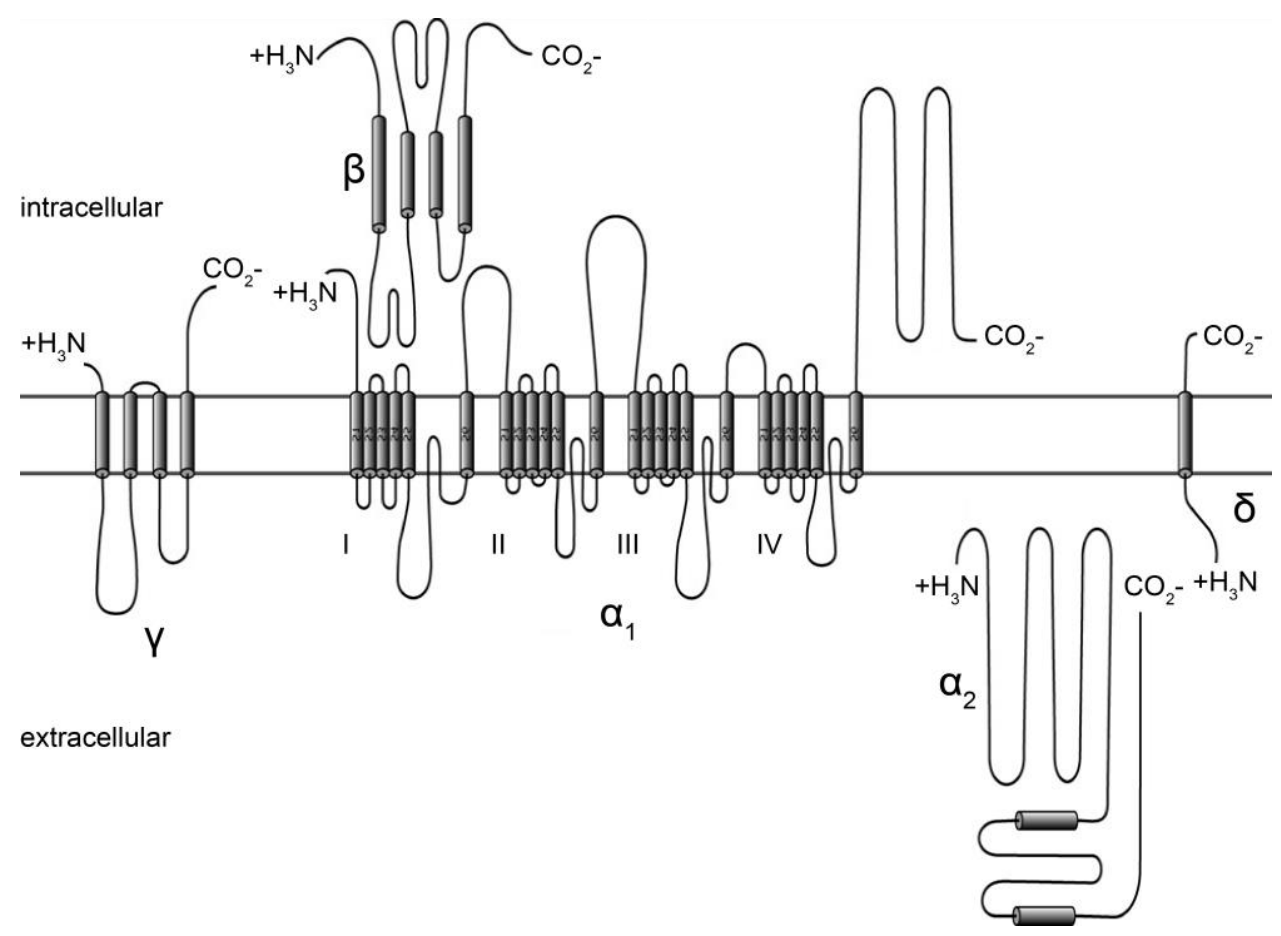

\section{Figure 4: Subunit composition and structure of voltage-gated $\mathrm{Ca}^{2+}$ channels}

Schematic illustrating the subunit composition and structure of VGCCs. The $\mathrm{Ca}_{\mathrm{v}} \alpha_{1}$ subunit is composed of four domains, each of which contains six transmembrane segments. The voltage sensor is thought to be located in segment $S 4$. The $\operatorname{Cav} \beta$ subunit is intracellular and binds to the $\operatorname{Cav} \alpha_{1}$ subunit via the $\alpha$ interaction domain. $\mathrm{Ca}_{v} \alpha_{2} \delta$ is composed of two parts, the membrane-bound $\mathrm{Ca}_{\mathrm{v}} \delta$ and the extracellular $\mathrm{Cav}_{2} \alpha_{2}$. Cav $\gamma$ is a transmembrane subunit. Modified from (Catterall et al., 2005).

$\operatorname{Cav} \beta$ subunits are entirely intracellular domains encoded by four different genes, coexpressed with the $\mathrm{Cav}_{1} \alpha_{1}$ subunits and crucial for their trafficking and function (reviewed in Buraei and Yang, 2010). They bind to the intracellular loop between domains I and II of the Cavo $\alpha_{1}$ subunit (Pragnell et al., 1994). These subunits are part of the membrane-associated guanylate kinase proteins family (Hanlon et al., 1999), which organize intracellular signaling pathways with multiple protein-protein interaction domains. Therefore, $\operatorname{Cav} \beta$ subunits have been associated to various modulatory effects. They were proposed to increase the transport of Cav $\alpha_{1}$ to the plasma membrane via the $\alpha$ interacting domain (AID) (Bichet et al., 2000; Gebhart et al., 2010); to increase the open probability of the $\mathrm{Ca}^{2+}$ channel (Neely et al., 1993); and to change their voltage-dependence of activation (Jones et al., 1998) and inactivation (Meir and Dolphin, 2002). In IHCs, the $\beta 2$ subunit is the main $\operatorname{Cav} \beta$ subunit and its genetic deletion involved deficits in cochlear amplification and sound encoding. IHCs of mice lacking Cav $\beta 2$ in all tissues, but the heart, exhibited a reduced membrane expression of Cav1.3 $\mathrm{Ca}^{2+}$ channels, resulting in a decreased synaptic $\mathrm{Ca}^{2+}$ influx and exocytosis. Moreover, $\mathrm{Cav} \beta 2$ are essential for development since the efferent innervation persisted onto the IHCs (Neef et al., 2009). 
Apparently, $\mathrm{Cav} \beta 3$ and $\mathrm{Cav} \beta 4$ are also expressed in IHCs (Kuhn et al., 2009; Neef et al., 2009) but only a mutation of Cav $\beta 4$ affected Cav1.3 expression at the plasma membrane (Kuhn et al., 2009).

The $\mathrm{Cav}_{2} \delta$ subunits are members of the glycosylphosphatidylinositol-anchored family that are expressed throughout the nervous system. They are encoded from four genes and consist of two parts, $\mathrm{Ca}_{v} \alpha 2$ and $\mathrm{Cav} \delta$, translated from the same mRNA and post-translationally cleaved. The $\mathrm{Cav} \delta$ subunit is a single transmembrane segment and linked to the extracellular $\mathrm{Cav}_{2} \alpha_{2}$ subunit by a disulfide bond (Davies et al., 2010). They were shown to associate with other $\mathrm{Cav} \alpha_{1}$ subunits such as $\mathrm{Cav} \alpha_{1 \mathrm{~A}}, \mathrm{Cav} \alpha_{1 \mathrm{~B}}$, and $\mathrm{Cav} \alpha_{1 \mathrm{C}}$ (Liu et al., 1996; Witcher et al., 1993). The main function of $\mathrm{Cav} \alpha_{2} \delta$ is to augment the abundance of $\mathrm{Cav} \alpha_{1}$ at the plasma membrane (reviewed in Dolphin, 2013). This increase in was shown to result from a reduced turnover of the channels resulting in a higher stability at the membrane and a chaperone-like function of $\mathrm{Cava}_{2} \delta$ (Bernstein and Jones, 2007; Gao et al., 2000; Hoppa et al., 2012). A mutant mouse line lacking the expression of full length $\mathrm{Cav}_{2} \alpha_{2} \delta_{2}$, exhibited mild hearing impairment and altered cochlear amplification along with decreased $\mathrm{Ca}^{2+}$ currents and voltage sensitivity in IHCs. These results proposed that $\mathrm{Cav}_{2} \alpha_{2} \delta_{2}$ is the predominant subunit of Cav1.3 in IHC (Fell et al., 2016).

Cav $\gamma$ subunits are encoded from height different genes and are believed to possess four transmembrane domains (Green et al., 2001; Jay et al., 1990), however their role has not been as much elucidated as those of the other subunits. The most consistent effect of VGCC modulation by $\mathrm{Cav} \gamma$ is the induction of a positive shift of voltage-dependence of activation (reviewed in Black, 2003). However, their role is still controversial due to their rare detection as part of VGCCs. These subunits also regulate $\alpha$-amino-3-hydroxy-5-methyl-4isoxazolepropionic acid (AMPA) receptor trafficking, localization and modulation (Nicoll et al., 2006; Osten and Sternbach, 2006).

VGCCs show two types of inactivation: voltage-dependent inactivation (VDI) and $\mathrm{Ca}^{2+}$ dependent inactivation (CDI; Brehm and Eckert, 1978). VDI comes from the capability of VGCCs to inactivate in response to voltage-dependent conformational changes. The structural components of VGCCs responsible for VDI depend on interactions between the pore of the VGCC and the AID region of the cytosolic I-II loop of Cava1 (Dafi et al., 2004; Stotz and Zamponi, 2001). The VDI response can be read as a response of the AID-Cav $\beta$ complex to block the channel pore (Tadross et al., 2010). The amount of VDI differs markedly depending 
on the $\mathrm{Cav} \alpha 1$ and $\beta$ subunits. Interestingly, $\mathrm{Ca}_{\mathrm{v}} 1.3$ channels exhibit an additional shield region within the S6 segment and are therefore more resistant to VDI (Tadross et al., 2010). Additionally, VGCC inactivate upon $\mathrm{Ca}^{2+}$ entry. This CDI restricts excessive $\mathrm{Ca}^{2+}$ influx in response to electrical activity. Calmodulin $(\mathrm{CaM})$ preassociates with L-, P/Q-, and R-type $\mathrm{Ca}^{2+}$ channels even in the absence of $\mathrm{Ca}^{2+}$ (Erickson et al., 2001; Pitt et al., 2001) and, once $\mathrm{Ca}^{2+}$ bound, is responsible for CDI by binding to the IQ-motif of the Caval subunit (Peterson et al., 1999; Qin et al., 1999; Zühlke et al., 1999). CaM possesses two distinct $\mathrm{Ca}^{2+}$ binding regions at the N-terminal that senses global increases in $\mathrm{Ca}^{2+}$ concentration (Cens et al., 2006), and Cterminal lobe senses local, sharp $\mathrm{Ca}^{2+}$ concentrations rising in the $\mathrm{Ca}^{2+}$ domain at the mouth of the channel (DeMaria et al., 2001; Yang et al., 2006). CDI can be regulated in a variety of ways. It can be influenced by the diverse types and alternative splicing of $\mathrm{Cav} \alpha_{1}$ subunits (Shen et al., 2006), by the type of Cav subunit co-expressed with the channel as well as by other EF-hand $\mathrm{Ca}^{2+}$ binding proteins (Cui et al., 2007).

\subsubsection{3 $\mathrm{Ca}^{2+}$ signaling at inner hair cell active zones}

The molecular mechanisms responsible for anchoring the $\mathrm{Ca}^{2+}$ channels at the IHC AZ have not yet been fully elucidated. IHCs lacking Bassoon exhibited an impaired $\mathrm{Ca}^{2+}$ channel clustering at the AZs, reducing the whole-cell and synaptic $\mathrm{Ca}^{2+}$ influx. However, a direct interaction was not found and the involvement of the loosened ribbon anchorage in this phenotype was not addressed (Frank et al., 2010). A promising candidate is rab3-interacting molecule (RIM). RIMs are multi-domain proteins that positively regulate the number of $\mathrm{Ca}^{2+}$ channels at the presynaptic AZ (Gebhart et al., 2010; Han et al., 2015; Kaeser et al., 2011; Kintscher et al., 2013; Kiyonaka et al., 2007). It has been shown that RIM2 $\alpha$ and RIM2 $\beta$ promote a large complement of synaptic $\mathrm{Ca}^{2+}$ channels at IHC AZs and are required for normal hearing. Indeed, the AZs of RIM2 $\alpha$-deficient IHCs were shown to cluster fewer synaptic Cav1.3 $\mathrm{Ca}^{2+}$ channels, resulting in reduced synaptic $\mathrm{Ca}^{2+}$ influx associated with a reduction of exocytosis, while the apparent $\mathrm{Ca}^{2+}$ dependence of exocytosis was unchanged. Moreover, auditory brainstem responses (ABRs) indicated a mild hearing impairment (Jung et al., 2015). Afterwards, an interaction of the $\mathrm{C}_{2} \mathrm{~B}$ domain of RIM2 $\alpha$ and RIM $3 \gamma$ with the C-terminus of the pore-forming $\alpha$-subunit of Cav1.3 channels was reported, positively regulating their plasma membrane expression in HEK293 cells (Picher et al., 2017a). The interacting partner of RIM, RIM binding protein 2 (RIM-BP2), was also shown to positively regulate the number of synaptic Cav1.3 
channels and thereby facilitate SV release. Its disruption resulted in a mild deficit of synaptic sound encoding (Krinner et al., 2017).

Interestingly, VGCCs can likely be opened at resting membrane potentials (Robertson and Paki, 2002), and additionally $\mathrm{Ca}^{2+}$ ions can continuously enter the cell through MET channels (Lumpkin and Hudspeth, 1995). To be able to retransmit with high fidelity the soundevoked stimulation, the IHCs need to spatiotemporally modulate the $\mathrm{Ca}^{2+}$ signals with great precision. $\mathrm{Ca}^{2+}$ ATPase at the cell membrane, as well as thapsigargin sensitive intracellular stores and mitochondria supposedly contribute to the regulation of cytosolic $\left[\mathrm{Ca}^{2+}\right]$ at least in immature IHCs (Kennedy, 2002). However, these mechanisms may not be sufficiently fast and localized close enough to the AZs to rapidly terminate sharp $\left[\mathrm{Ca}^{2+}\right]_{\mathrm{i}}$ increase mediated by synaptic $\mathrm{Ca}^{2+}$ channel clusters (Roberts, 1994). Therefore, IHCs are equipped with mobile proteinaceous $\mathrm{Ca}^{2+}$ buffers, parvalbumin- $\alpha$, calretinin and calbindin (Hackney et al., 2005). Their concentrations as well as their $\mathrm{Ca}^{2+}$ association and dissociation kinetics play a major role in determining both the amplitude and the spatiotemporal properties of the presynaptic $\mathrm{Ca}^{2+}$ signals that govern neurotransmitter release (Edmonds et al., 2000; Neher and Augustine, 1992; Roberts, 1993). They have been proposed to regulate presynaptic IHC function for metabolically efficient sound coding (Pangrsic et al., 2015). IHCs lacking all three proteins showed excessive exocytosis during prolonged depolarizations, despite enhanced $\mathrm{Ca}^{2+}$ dependent inactivation of their $\mathrm{Ca}^{2+}$ currents. Synaptic sound encoding was largely unaltered, suggesting that the excess exocytosis primarily occurred extrasynaptically (Pangrsic et al., 2015).

$\mathrm{Ca}^{2+}$ binding proteins (CaBPs), too, are members of the EF-hand $\mathrm{Ca}^{2+}$ binding proteins family. They are encoded by eight genes and subdivided into two subfamilies with distinct intracellular localization profiles (Haeseleer et al., 2000). All of them are comprised of two lobes, each bearing a pair of EF-hands connected by an inter-lobe flexible a-helix linker region. Unlike CaM, EF-hand in the CaBP N-lobe is not binding $\mathrm{Ca}^{2+}$. In contrast to ubiquitous $\mathrm{CaM}$ expression in all eukaryotic cells, CaBPs are restricted to sensory cells and neurons (Haeseleer et al., 2000). CaBPs might perturb the normal binding of CaM to VGCCs, probably by competing with binding to the IQ domain and preventing CDI (Cui et al., 2007; Schrauwen et al., 2012; Yang et al., 2006). Given the advantage of CaBPs over CaM that results in preventing $\mathrm{CDI}$ despite similar $\mathrm{K}_{\mathrm{d}}$-values, $\mathrm{CaBPs}$ can be assumed to reside in closer proximity to the IQdomain. Whether the competition between CaBPs and CaM for the IQ domain depends only on $\mathrm{Ca}^{2+}$ requires further investigation (Tadross et al., 2010). Among CaBPs, CaBP1 is the most 
heavily investigated isoform in respect to VGCC function: $\mathrm{CaBP} 1$ upregulates $\mathrm{Ca}^{2+}$ dependent facilitation and voltage-dependent inactivation of Cav1.2 channels (Oz et al., 2011; Zhou et al., 2004). To date only CaBP4 was reported to be required for normal vision, was localized to photoreceptor synaptic terminals and, apparently, is involved in rod and cone synapse formation and function (Haeseleer et al., 2004; Zeitz et al., 2006). In addition, CaBP5 is expressed in rod bipolar cells and some cone bipolar cell types, but is not strictly required for vision (Rieke et al., 2008). IHCs display considerably weak CDI in comparison with neurons or cell lines. In mouse IHCs, CaBP1-5 were detected by immunohistochemistry, but their respective function is under current investigation. CaBP2 was proven to be a deafness gene (Schrauwen et al., 2012; Picher et al., 2017b). Cabp2 loss showed impaired ABRs, enhanced $\mathrm{Ca}^{2+}$ channel inactivation and reduced spontaneous and sound-evoked firing rates. CaBP2 was proposed to inhibit Cav1.3 $\mathrm{Ca}^{2+}$ channel inactivation, and thus sustains the availability of $\mathrm{Cav} 1.3 \mathrm{Ca}^{2+}$ channels for synaptic sound encoding (Picher et al., 2017b).

Together, the clustering of VGCCs at AZs and the $\mathrm{Ca}^{2+}$ binding to mobile $\mathrm{Ca}^{2+}$ buffers establish domains of locally elevated $\left[\mathrm{Ca}^{2+}\right]_{\mathrm{i}}$. For any effector of $\left[\mathrm{Ca}^{2+}\right]_{\mathrm{i}}$, like the vesicular $\mathrm{Ca}^{2+}$ sensor for fusion, their spatial relation to these domains is of considerable functional significance. In mature IHCs, $\mathrm{Ca}_{\mathrm{v}} 1.3 \mathrm{Ca}^{2+}$ channels localize at mature presynaptic AZs underneath synaptic ribbons. This tight organization importantly decreases the distance that incoming $\mathrm{Ca}^{2+}$ has to travel to reach the $\mathrm{Ca}^{2+}$ sensors on the synaptic vesicles and thus allows for high sensitivity to changes in membrane potential. In the scenario of nanodomain control of exocytosis (Neher, 1998), in which the opening of one or few $\mathrm{Ca}^{2+}$ channels would trigger the release of a vesicle, the $\mathrm{Ca}^{2+}$ sensor is located very close to the channel(s) $(\approx 20 \mathrm{~nm})$, and will be exposed to sharp $\left[\mathrm{Ca}^{2+}\right]_{\mathrm{i}}$ increment of several tens to hundreds of $\mu \mathrm{M}$ upon channel opening (Roberts, 1994). Experimentally, due to the small coupling distance, it can be characterized by the fact that only very 'fast' buffers (high association rate, $\mathrm{k}_{\text {on }}$; e.g. 1,2- bis (2-aminophenoxy) ethane- $N, N, N 9, N 9$-tetraacetate (BAPTA); (Naraghi, 1997) are effective in capturing $\mathrm{Ca}^{2+}$ ions before they reach the $\mathrm{Ca}^{2+}$ sensor and hence in inhibiting stimulussecretion coupling. One advantage is that the nanodomain control would allow to act rapidly upon channel opening, reflected in exocytic delays in the range of hundreds of microseconds (Yamada and Zucker, 1992). Exposing the $\mathrm{Ca}^{2+}$ sensor of fusion to the saturating $\left[\mathrm{Ca}^{2+}\right]$ of the nanodomain, would make the timing of synaptic sound encoding less sensitive to stimulus intensity, but would be less reliable since the opening of $\mathrm{Ca}^{2+}$ channels is stochastic (Moser et al., J Physiol 2006). In the microdomain control of exocytosis (Augustine et al., 2003; Neher, 1998), the opening of several $\mathrm{Ca}^{2+}$ channels trigger the release of one vesicle, the $\mathrm{Ca}^{2+}$ sensor 
is placed farther away from the channels $(\approx 100-200 \mathrm{~nm})$, and will experience smaller $\left[\mathrm{Ca}^{2+}\right]_{\mathrm{i}}$ elevations, a longer time to peak $\left[\mathrm{Ca}^{2+}\right]_{\mathrm{i}}$, and an increased buffering susceptibility due to the longer coupling distance. It can be characterized by the effectiveness of 'slow' buffers (lower association rate, kon; e.g. ethylene gly- col-bis-(2-aminoethyl)-N,N,N',N'-tetraacetic acid (EGTA) (Naraghi, 1997). One advantage is that this microdomain control is more reliable than the nanodomain control since it builds on more channels, but is slower since it needs more $\mathrm{Ca}^{2+}$ influx to reach the required local $\left[\mathrm{Ca}^{2+}\right]$ at the sensor. However, if several channels in a cluster open more or less simultaneously, the $\mathrm{Ca}^{2+}$ increments would sum up, and the required concentration at the sensor would be reached more quickly. Thus, rapid exocytosis is also achievable with a microdomain control of transmitter release, as it is the case at immature the calyx of Held synapse (Borst and Sakmann, 1996).

Those two extreme scenarios (Figure 5) differ in term of apparent $\mathrm{Ca}^{2+}$ cooperativity of exocytosis when the the number of open $\mathrm{Ca}^{2+}$ channels is varied. A linear increase in the exocytic response is expected for a nanodomain control of exocytosis due to the dominance of one $\mathrm{Ca}^{2+}$ channel in governing the fusogenic $\mathrm{Ca}^{2+}$, whereas a supra-linear increase is predicted for a microdomain control reflecting intrinsic high cooperativity of exocytosis (Matveev et al., 2009). The preferred scenario of coupling between $\mathrm{Ca}^{2+}$ channels and sensors at IHC synapses is the nanodomain-like control, due to evidences found by correlating presynaptic $\mathrm{Ca}^{2+}$ influx with exocytosis (Brandt et al., 2005; Pangrsic et al., 2015; Wong et al., 2014) or EPSC charges (Goutman and Glowatzki, 2007; Keen and Hudspeth, 2006). These post synaptic responses are carried out by the SGNs that convey the sound information via the auditory nerve to the brain stem. 


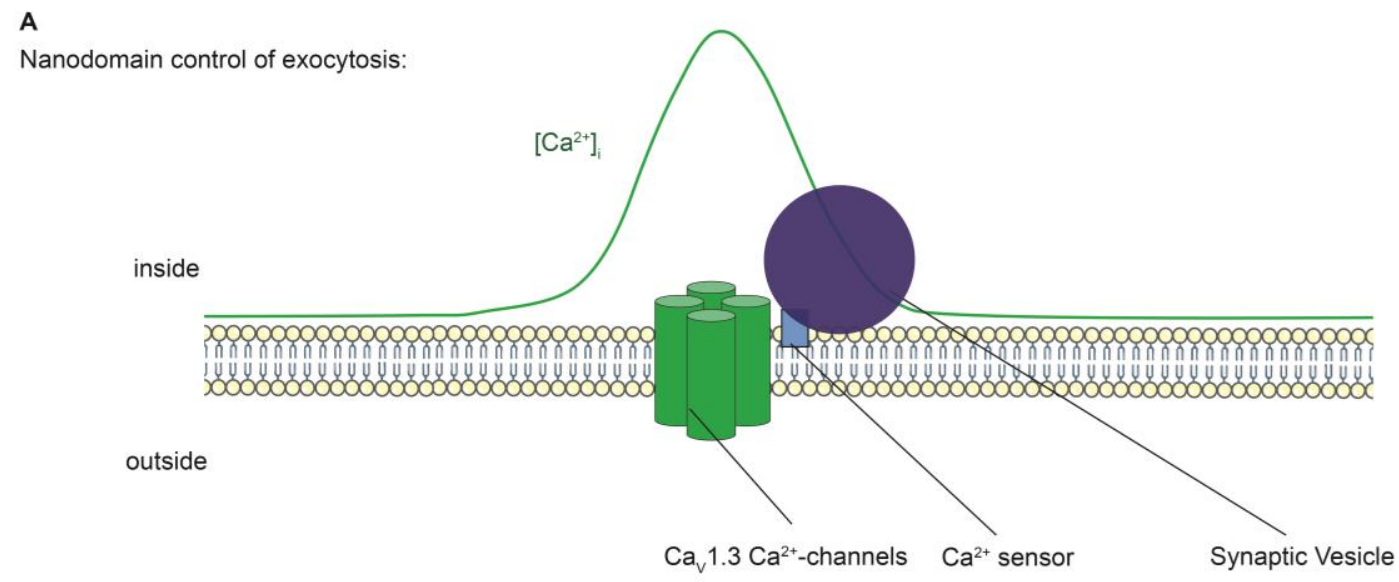

\section{B} Microdomain control of exocytosis:

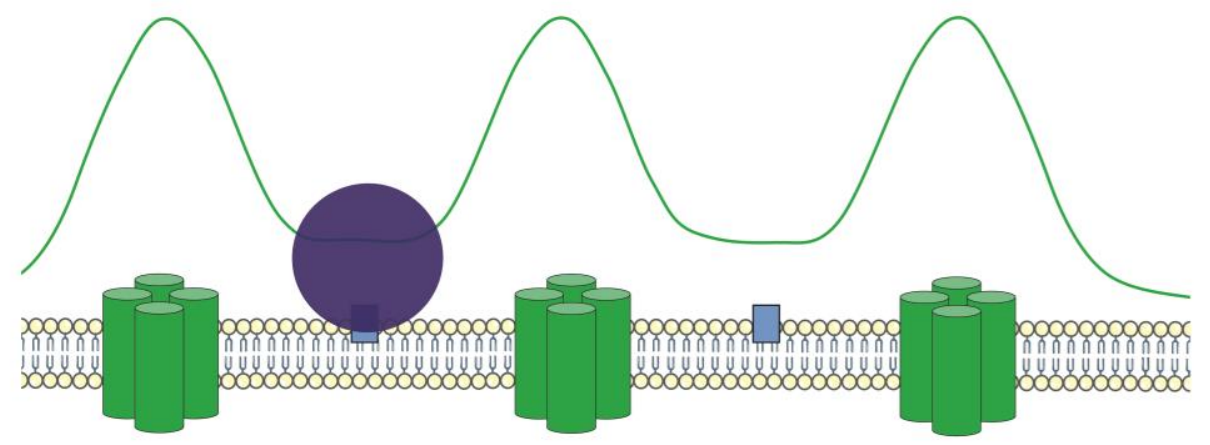

Figure 5: Coupling between voltage-gated $\mathrm{Ca}^{2+}$ channels and vesicular release sites

(A) Nanodomain control of exocytosis: The $\mathrm{Ca}^{2+}$ sensors are located very close from the $\mathrm{Ca}^{2+}$ channels $(\sim 20 \mathrm{~nm})$, and therefore would be exposed to sharp $\left[\mathrm{Ca}^{2+}\right]_{\mathrm{i}}$ increment upon channel opening. The opening of one or few channels would trigger the release of a vesicle. In such a scenario, the vesicle release is linearly dependent on the number of open $\mathrm{Ca}^{2+}$ channels. (B) Microdomain control of exocytosis: The $\mathrm{Ca}^{2+}$ sensors are located further away from the $\mathrm{Ca}^{2+}$ channels $(\sim 100-200 \mathrm{~nm})$, and will be exposed to smaller $\left[\mathrm{Ca}^{2+}\right]_{i}$ elevations. The opening of more $\mathrm{Ca}^{2+}$ channels would trigger the release of a vesicle. Hence, the release of vesicles is non-linearly dependent on the number of open $\mathrm{Ca}^{2+}$ channels. Not to scale.

\subsubsection{Encoding of sound intensity in spiral ganglion neurons}

In brief, mature IHCs are connected with about 10 to 20 type I SGNs (hereafter "SGN") in a 1:1 connection. These SGNs are the first afferent neurons of the auditory system, and make up around $95 \%$ of the ganglion, while the remaining ones are type II and contact individually a dozen of OHCs (Kiang et al., 1982; Spoendlin, 1969, 1972). The IHC graded receptor potential governs the $\mathrm{Ca}^{2+}$ dependent release of neurotransmitter that activates glutamate receptors on the afferent bouton resulting in excitatory postsynaptic potentials (EPSPs) in the SGN (Corey and Hudspeth, 1979; Robertson and Paki, 2002; Sewell, 1984). When a large enough number of receptors have been activated, the resulting currents depolarize the cell beyond threshold, 
thus triggering an action potential. The function of these synapses is similar to an analogdigital converter, in which an analog sensory signal is encoded as a digital code. The first heminode was reported to reside just beneath habenulae perforatae not far from the IHCs suggesting that the action potential is triggered nearby the small postsynaptic bouton, reducing the duration of membrane charging to the threshold of spiking. These features guarantee that SGNs can respond to the presynaptic stimulus with great temporal accuracy. The other important feature of SGNs is their phasic excitability. SGNs only spike once or few times due to their expression of voltage-gated $\mathrm{K}^{+}-(\mathrm{Kv})$ channels. This together with refractoriness makes them able to filter out some other EPSPs which are not elicited by the well-timed presynaptic releasing of neurotransmitters, thereby enhancing the precision of signal transmission in SGNs (for review see Davis and Crozier, 2016; Rutherford and Moser, 2016).

During spontaneous firing (spontaneous rate: SR, measured as the firing rate in the absence of sound), more than $90 \%$ of the spontaneous EPSPs can generate an action potential (Rutherford et al., 2012). The spiking rate of SGNs increases up to several hundreds of $\mathrm{Hz}$ in response to increasing sound intensity and is essentially limited by the neuron refractoriness. However, mammalian auditory system can encode sound pressures varying by 6 orders of magnitude. Despite the nonlinear compression of basilar membrane, SGNs still need to be able to respond to sound pressures that vary over thousand-fold. In mammalian cochlea, SGNs vary in their response to sound stimulation. Their firing rate, response threshold and dynamic range are highly heterogeneous, even among neurons showing similar $\mathrm{C}_{\mathrm{f}}$ (indicating that they are innervating the same IHC or ones in close proximity) (Liberman, 1978). Therefore, SGNs work as different channels to the brain enabling the response of wide ranges of sound pressure. Three response patterns for sound levels of the SGNs were identified: "saturating," "slopingsaturation," and "straight" modes. The SGNs for the saturating mode have the lowest acoustic threshold $(\sim 10-30 \mathrm{~dB})$ to fire action potentials but their firing rate does not increase after a certain sound intensity. The fibers presenting sloping-saturation (getting slower) and straight mode (linear) have higher thresholds; however, their firing rate never saturate. The SR showed negative relationship with response threshold in cat (Liberman, 1978), guinea pig (Winter et al., 1990), gerbil (Ohlemiller and Echteler, 1990), rat (Barbary, 1991) and mouse (Taberner and Liberman, 2005). It was found that, in cat, SGNs can be roughly divided into three groups based on their SR (high, medium, low), each corresponding to a different sensitivity (Liberman, 1978). Later, it was observed that the medium- and low-SR rate (higher threshold) neurons preferentially innervate the neural/modiolar (facing afferent fibers) side of IHCs, while high SR neurons innervated the abneural/pillar (facing OHCs) side (Liberman, 1982). 
These findings suggest the existence of a segregation of neural responses properties at the synaptic level.

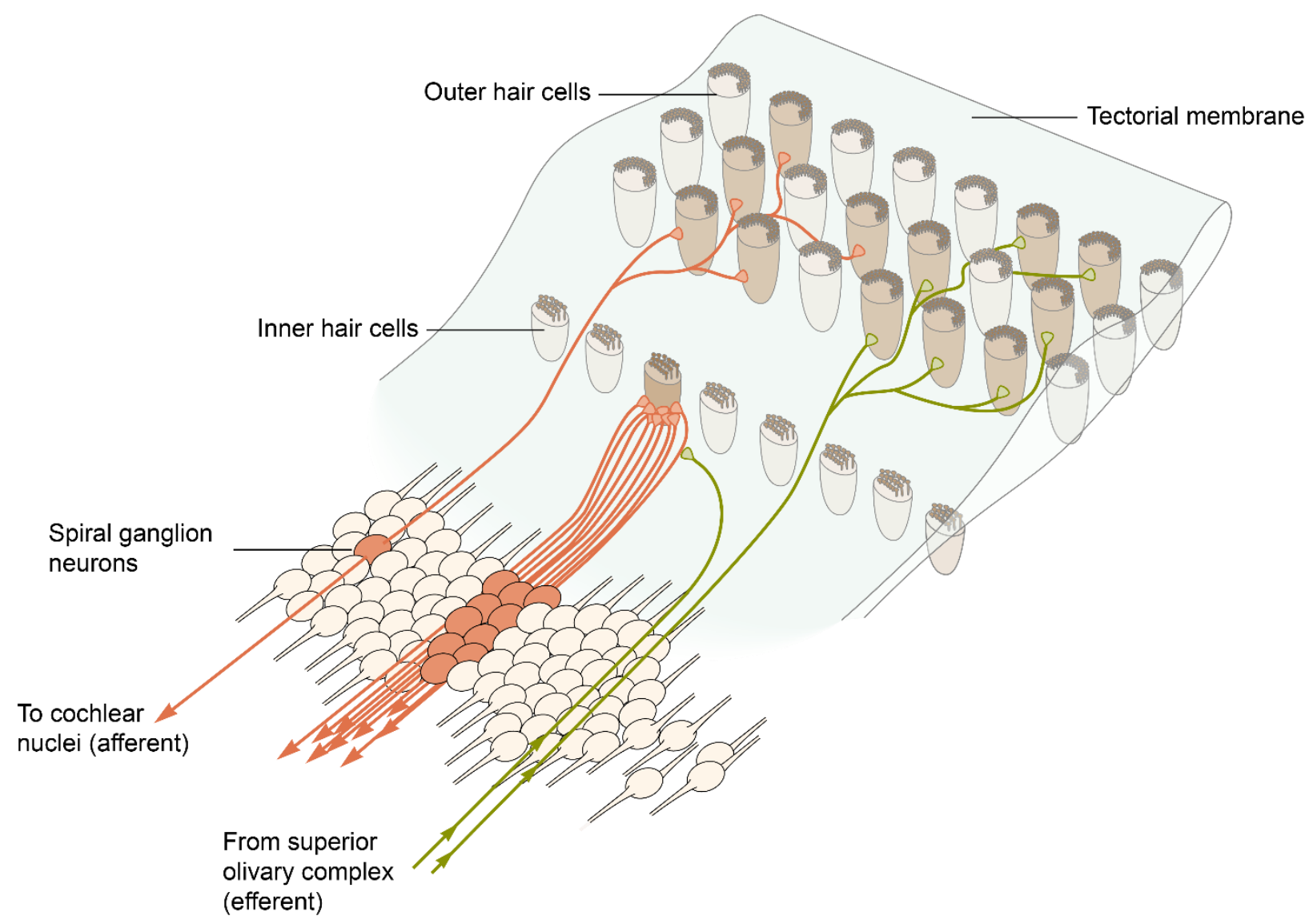

Figure 6: Innervation pattern of mature cochlear hair cells

Illustration displaying the innervation pattern of cochlear hair cells in mature mice. Each IHC is the sole input of a dozen of type I SGNs. Fewer type II SGNs individually transmit signal from several OHCs. Efferent axons largely and directly innervate the OHCs, whereas this innervation is sparser at the level of the IHCs, and make input with the afferent. Modified from (Kandel, 2012).

\subsubsection{Spatial heterogeneity of synaptic $\mathrm{Ca}^{2+}$ influx properties at inner hair cells}

One attractive mechanism driving the synapse-based segregation of SGN firing behaviors is the diversity of IHC AZ properties. First studies (Frank et al., 2009; Meyer et al., 2009) established confocal $\mathrm{Ca}^{2+}$ imaging at IHCs of mice after the onset of hearing using low-affinity $\mathrm{Ca}^{2+}$ indicator and strong buffering condition allowing to characterize spatially confined $\mathrm{Ca}^{2+}$ domains. These localized domains of high $\left[\mathrm{Ca}^{2+}\right]$ also occur at IHC synapses studied at 
physiological-like $\mathrm{Ca}^{2+}$ concentrations and buffering (Neef et al., 2018). Several pieces of evidence strongly suggested that these observed $\mathrm{Ca}^{2+}$ microdomains arose from $\mathrm{Ca}^{2+}$ influx through synaptic $\mathrm{Cav} 1.3-\mathrm{Ca}^{2+}$ channel clusters at the ribbon synapses. First, they occurred at sites marked by a ribbon-labeling CtBP2/RIBEYE-binding fluorescent peptide (Zenisek et al., 2004). Moreover, These $\mathrm{Ca}^{2+}$ microdomains were not observable when removing extracellular $\mathrm{Ca}^{2+}$. Additionally, their voltage-dependence mimicked that of the whole-cell $\mathrm{Ca}^{2+}$ current. Finally, inhibition of $\mathrm{Ca}^{2+}$ induced $\mathrm{Ca}^{2+}$ release by ryanodine or intracellular $\mathrm{Cs}^{+}$did not reduce the amplitude of the $\mathrm{Ca}^{2+}$ microdomains. A great variation in the voltage dependence (voltage of half-maximal activation) of the $\mathrm{Ca}^{2+}$ fluorescence increment among AZs of an individual IHC was shown, which was four times larger than that of the whole-cell current compared among the same experiments. In addition to these shifts in the activation, a large fluorescence variability of $\mathrm{Ca}^{2+}$ microdomains has also been found for strong depolarizations when $\mathrm{Ca}^{2+}$ channel activation saturates. Finally, the study did not support a major contribution of $\mathrm{Ca}^{2+}$ signal augmentation by local differences in $\mathrm{Ca}^{2+}$ buffering/sequestration, or the $\mathrm{Ca}^{2+}$ channel open probability among IHC synapses (Frank et al., 2009). Each presynaptic AZ is controlled by a common IHC receptor potential and provides the sole excitatory input to "its" postsynaptic SGN. ${ }^{2+}$ This study proposed that the IHC differently adjusts the gating and number of $\mathrm{Ca}^{2+}$ channels at each AZ, establishing distinct voltage dependences of transmitter release, being a putative presynaptic mechanism for driving different SGN firing characteristics.

To spatially characterize these observations, a later study (Ohn et al., 2016) used a spinning disk confocal microscope, a multi-beam scanning system permitting fast 3D-liveimaging allowing an analysis of most if not all AZ properties as a function of position within an individual IHC. In parallel, a semi quantitative confocal microscopy of immunolabelled IHC synapses was performed. The study suggested that IHCs spatially decompose sound intensity information into different outputs by varying the maximal $\mathrm{Ca}^{2+}$ influx and the voltagedependent activation among their AZs along the pillar/modiolar IHC axis. These two influx characteristics exhibited opposite spatial gradients. Modiolar AZs showed bigger ribbons, more $\mathrm{Ca}^{2+}$ channels, and on average, a tendency towards a stronger maximal $\mathrm{Ca}^{2+}$ influx than the $\mathrm{AZs}$ from the opposite side. A greater number of $\mathrm{Ca}^{2+}$ channels is expected to trigger more spontaneous and evoked release, and a stronger spontaneous and evoked firing rate at the postsynapse (Robertson and Paki, 2002). However, they found that modiolar AZs, on average, presented a voltage operating range shifted towards more positive potentials as compared to the pillar side AZs, consistent with the idea that modiolar synapses drive low SR- high-threshold SGNs (Figure 7). These AZs holding more $\mathrm{Ca}^{2+}$ channels may support higher maximal rates of 
transmitter release, which could explain the higher susceptibility of their associated afferent fibers to excitotoxic insult during acoustic overexposure.

However, whereas innervation of functionally distinct classes of type I SGNs at the level of IHC was established in cats, data revealing the presynaptic heterogeneity were obtained from mice where a different afferent connectivity is conceivable. Moreover, an immaturity phenotype cannot be ruled out for several of the mouse studies since the experiments were done shortly after the hearing onset. Indeed a recent study based on immunostaining analysis showed an inversion of the ribbon and AMPAR patch modiolar/pillar gradients from P21 of age, gradient being maximal and unchanged from P28 (Liberman and Liberman, 2016). Additionally, the variability in the operating voltage range of $\mathrm{Ca}^{2+}$ influx among synapses of one-half of the cell (modiolar or pillar) could exceed the difference between the average pillar and modiolar synapses. The SGNs with identical frequency tuning but different sound-response properties apparently receive input from the same IHC and collectively vehicle acoustic information across the entire audible range of sound pressures to the brain. Most probably a combination of presynaptic, postsynaptic and efferent mechanisms underlie this diversity of SGN soundresponse properties.

\subsection{Aim of this work}

The first part of my thesis focused on assessing the role of the synaptic ribbon in sound encoding. Taking advantage of mice $\mathrm{KO}$ for Ribeye A domain and consequently lacking ribbons, I together with collaborators characterized the morphology of the ribbonless synapses by immunofluorescence and electron microscopy. The physiology of sound encoding was then studied by performing patch-clamp/ $\mathrm{Ca}^{2+}$ imaging of IHCs and in vivo extracellular recordings of SGNs. The second part of my thesis aimed to decipher the cellular mechanisms contributing to the diversity of SGN firing behaviors thought to underlie the wide dynamic range of sound encoding. Performing immunostainings and patch-clamp recordings combined with fast live $\mathrm{Ca}^{2+}$ imaging, and with help from collaborators, I tested two different candidate mechanisms. I investigated if: 1) Pou4f1, a type I SGN transcription factor, postnatally expressed almost exclusively ( $95 \%)$ in a subpopulation of SGNs targeting the modiolar face of the IHCs, and 2) the mechanisms involved in IHC planar polarity governing hair bundle orientation and apical surface asymmetry, are instructing the basolateral gradient of synapse properties, putatively responsible for the diverse firing properties of type I SGNs. 


\section{Modiolar side}

\section{Postsynaptic:}

High threshold / Low spontaneous rate fibers with smaller caliber

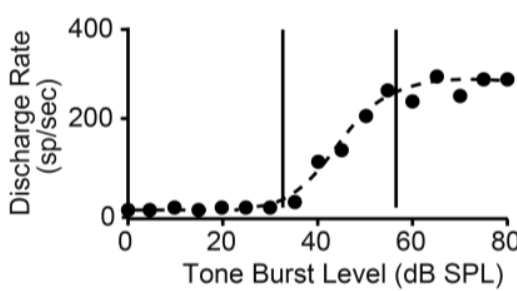

Presynaptic:

Larger AZs

with a more depolarized activation of $\mathrm{Ca}^{2+}$ channels

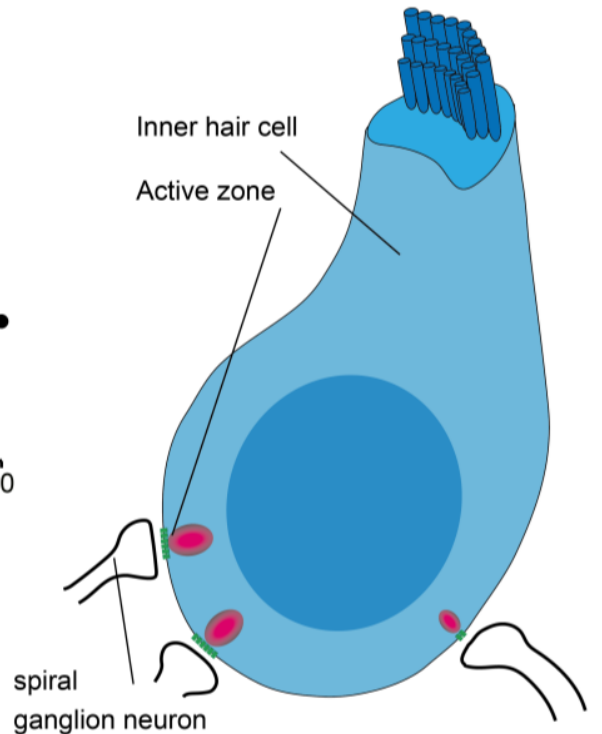

Pillar side

Postsynaptic:

Low threshold / high spontaneous rate fibers with larger caliber

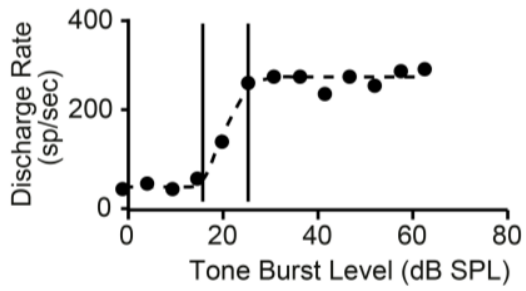

Presynaptic:

Smaller AZs

with a more hyperpolarized activation

of $\mathrm{Ca}^{2+}$ channels

Figure 7: Basolateral gradient of inner hair cell synapse properties and associated fiber

\section{firing}

Illustration showing the IHC spatial synaptic connectivity. The low SR SGNs preferentially make a synapse with the modiolar side of the IHC, exhibiting large ribbons and $\mathrm{Ca}^{2+}$ channel clusters activated at more depolarized potentials than the ones on the pillar side, exhibiting smaller AZs and making contact with the high SR SGNs. Data taken from (Taberner and Liberman, 2005). Not to scale. 


\title{
3 Chapter 1: Published article
}

\section{"The synaptic ribbon is critical for sound encoding at high rates and with temporal precision"}

\author{
P. Jean ${ }^{*}$, D. Lopez de la Morena*, S. Michanski*, L.M. Jaime Tobón ${ }^{*}$, R. Chakrabarti, M.M.
}

Picher, J. Neef, S.Y. Jung, M. Gültas, S. Maxeiner, A. Neef,, C. Wichmann, N. Strenzke, C.

Grabner, and T. Moser

* Equal contributions

\section{Author Contributions:}

T.M., C.G., C.W., A.N., N.S. designed the study. P.J. performed immunohistochemistry and confocal immunofluorescence microscopy, patch-clamp, $\mathrm{Ca}^{2+}$ imaging, and computational modeling together with A.N.. D.L. performed in vivo extracellular recordings from single SGNs and AVCN neurons. S.M. performed conventional embedding and EM of random and serial sections and contributed to highpressure freezing. L.M.J.T. performed patch-clamp capacitance measurements. R.C. performed highpressure freezing, freeze-substitution and electron tomography. M.M.P. performed patch-clamp capacitance measurements. J.N. performed immunohistochemistry, confocal and STED immunofluorescence microscopy, and co-supervised P.J. and L.M.J.T.. S.Y.J. performed immunohistochemistry and confocal immunofluorescence microscopy. M.G. contributed a statistical analysis of EM data. S.M. contributed mutant mice and genetic expertise, A.N. performed and supervised computational modeling. C.W. supervised EM and tomography. N.S. supervised in vivo extracellular recordings from single SGNs. T.M. co-supervised P.J. D.L. L.M.J.T. All authors performed analysis. T.M. and P.J. prepared the manuscript with contributions of all authors. 
*For correspondence: aneef@gwdg.de (AN); carolin.wichmann@med.unigoettingen.de (CW); nicola.strenzke@med.unigoettingen.de (NS); chad.grabner@mpibpc.mpg.de (CG);

tmoser@gwdg.de (TM)

${ }^{\dagger}$ These authors contributed equally to this work

Competing interests: The authors declare that no competing interests exist.

Funding: See page 33

Received: 07 July 2017 Accepted: 19 December 2017 Published: 12 January 2018

Reviewing editor: Christine Petit, Institut Pasteur, France

(c) Copyright Jean et al. This article is distributed under the terms of the Creative Commons Attribution License, which permits unrestricted use and redistribution provided that the original author and source are credited.

\section{The synaptic ribbon is critical for sound encoding at high rates and with temporal precision}

\author{
Philippe Jean ${ }^{1,2,3,4 \dagger}$, David Lopez de la Morena ${ }^{1,3,4 \dagger}$, Susann Michanski ${ }^{2,4,5,6 \dagger}$, \\ Lina María Jaime Tobón ${ }^{1,2,3,4,7,8+}$, Rituparna Chakrabarti ${ }^{2,3,4,5,6}$, \\ Maria Magdalena Picher ${ }^{1,2,4}$, Jakob Neef ${ }^{1,2,4,7,8}$, SangYong Jung 1,4,9, \\ Mehmet Gültas ${ }^{10}$, Stephan Maxeiner ${ }^{11}$, Andreas Neef ${ }^{12 *}$, Carolin Wichmann ${ }^{2,4,5,6 *}$ \\ Nicola Strenzke ${ }^{2,4,13 *}$, Chad Grabner ${ }^{1,4,7 *}$, Tobias Moser ${ }^{1,2,4,7,8,14 *}$
}

${ }^{1}$ Institute for Auditory Neuroscience and InnerEarLab, University Medical Center Göttingen, Göttingen, Germany; ${ }^{2}$ Collaborative Research Center, University of Göttingen, Göttingen, Germany; ${ }^{3}$ Göttingen Graduate School for Neurosciences and Molecular Biosciences, University of Göttingen, Göttingen, Germany; ${ }^{4}$ InnerEarLab, Department of Otolaryngology, University Medical Center Göttingen, Göttingen, Germany; ${ }^{5}$ Molecular Architecture of Synapses Group, Institute for Auditory Neuroscience, University Medical Center Göttingen, Göttingen, Germany; ${ }^{6}$ Institute for Biostructural Imaging of Neurodegeneration, University Medical Center Göttingen, Göttingen, Germany; ${ }^{7}$ Synaptic Nanophysiology Group, Max Planck Institute for Biophysical Chemistry, Göttingen, Germany; ${ }^{8}$ Auditory Neuroscience Group, Max Planck Institute for Experimental Medicine, Göttingen, Germany; ${ }^{9}$ Neuro Modulation and Neuro Circuitry Group, Singapore Bioimaging Consortium (SBIC), Biomedical Sciences Institutes, Singapore, Singapore; ${ }^{10}$ Department of Breeding Informatics, Georg-August-University Göttingen, Göttingen, Germany; ${ }^{11}$ Institute for Anatomy and Cell Biology, University of the Saarland, Homburg, Germany; ${ }^{12}$ Bernstein Group Biophysics of Neural Computation, Max Planck Institute for Dynamics and Self-Organization, Göttingen, Germany; ${ }^{13}$ Auditory Systems Physiology Group, Department of Otolaryngology, University Medical Center Göttingen, Göttingen, Germany; ${ }^{14}$ Center for Nanoscale Microscopy and Molecular Physiology of the Brain, University Medical Center Göttingen, Göttingen, Germany 


\section{Introduction}

Encoding and processing of sensory information in the ear and the eye rely on ribbon synapses. Described in the 1960s as an electron dense structure tethering a halo of vesicles (Sjostrand, 1958; Smith and Sjostrand, 1961), the function of the synaptic ribbon has remained enigmatic despite decades of work (recent reviews in Lagnado and Schmitz, 2015; Moser and Vogl, 2016; Safieddine et al., 2012; Wichmann and Moser, 2015). Approaches to ribbon function included studies that employed natural variation of ribbon size or abundance during diurnal cycle or hibernation (Hull et al., 2006; Mehta et al., 2013), photoablation (Mehta et al., 2013; Snellman et al., 2011) and genetic manipulation (Dick et al., 2003; Frank et al., 2010; Jing et al., 2013; Khimich et al., 2005; Lv et al., 2016; Maxeiner et al., 2016; Sheets et al., 2011; Van Epps et al., 2004). Mutations initially focused on the presynaptic scaffold protein bassoon that is required for ribbon anchorage to the AZ (Dick et al., 2003; Khimich et al., 2005) via interaction with RIBEYE (tom Dieck et al., 2005). However, bassoon also exerts direct effects on AZ function (Davydova et al., 2014; Hallermann et al., 2010; Mendoza Schulz et al., 2014) and, hence, distinguishing direct effects of bassoon deletion and those caused by ribbon loss remained challenging (Jing et al., 2013).

RIBEYE-disruption turned out to be difficult: it is transcribed from the same gene as CtBP2, an essential transcription factor, disruption of which causes embryonic lethality (Hildebrand and Soriano, 2002). Complete abolition of RIBEYE was hard to achieve in zebrafish (Lv et al., 2016; Van Epps et al., 2004) given their duplicated genome. In fact, despite targeting both ribeye genes, RIBEYE immunofluorescence remained present in the retina and hair cells displayed 'ghost ribbons': structures recognized by a synaptic vesicle-halo but lacking electron density (Lv et al., 2016). Complete disruption of RIBEYE expression and lack of retinal ribbons were recently reported in a mouse knock-out of the RIBEYE-specific exon (Maxeiner et al., 2016). This study proved that RIBEYE is required for ribbon formation in the mammalian retina and the observed ribbon loss grossly impaired glutamate release from bipolar cell terminals. The key conclusion was that ribbons help to couple voltage-gated $\mathrm{Ca}^{2+}$-channels to vesicular release sites to enable tight, so-called $\mathrm{Ca}^{2+}$-nanodomain control of exocytosis (Maxeiner et al., 2016), that was previously reported for ribbon synapses of ear and eye (Bartoletti et al., 2011; Brandt et al., 2005; Graydon et al., 2011; Jarsky et al., 2010; Johnson et al., 2017; Pangršič et al., 2015; Wong et al., 2014). By employing the most specific, yet chronic, manipulation of the ribbon, this functional study on ribbonless retinal rod bipolar cells also confirmed that RIBEYE/the ribbon promotes a large complement of vesicular release sites. However, the electrophysiology was performed on rod bipolar cells while the molecular anatomy (immunofluorescence) focused on rod photoreceptors. Since the structure and function of ribbons formed at these two different cell types are distinct, a simple structure-function model was not easy to derive from this study. Moreover, the consequences of ribbon loss remained to be investigated at the systems level. Here, we studied the effects of RIBEYE-disruption on synaptic sound encoding in the cochlea. Combining assessments of the molecular anatomy from electron and fluorescence microscopy with cell and systems physiology, we revealed a role for the synaptic ribbon in organizing the topography of the IHC AZ, in $\mathrm{Ca}^{2+}$-channel regulation and in vesicle replenishment. In summary, we demonstrate that the synaptic ribbon is important for sound encoding at high rates and with temporal precision at IHC synapses.

\section{Results}

\section{Genetic disruption of RIBEYE transforms ribbon-type AZs of IHC synapses into synaptic contacts with multiple small ribbonless AZs}

We first employed immunohistochemistry to study IHCs of 3-week-old RIBEYE knock-out mice $\left(\mathrm{RBE}^{\mathrm{KO} / \mathrm{KO}}\right.$ ), in which the unique A-domain exon of RIBEYE was deleted by Cre-mediated excision (described in Maxeiner et al., 2016). Next to the A-domain, RIBEYE contains a B-domain that is largely identical to the transcription factor CtBP2, which is spared by the genetic manipulation and used as a target in immunohistochemistry of ribbons and nuclei (Figure 1A, B; Khimich et al., 2005). 
eLife digest Our sense of hearing relies on our ears quickly and tirelessly processing information in a precise manner. Sounds cause vibrations in a part of the inner ear called the cochlea. Inside the cochlea, the vibrations move hair-like structures on sensory cells that translate these movements into electrical signals. These hair cells are connected to specialized nerve cells that relay the signals to the brain, which then interprets them as sounds.

Hair cells communicate with the specialized nerve cells via connections known as chemical synapses. This means that the electrical signals in the hair cell activate channel proteins that allow calcium ions to flow in. This in turn triggers membrane-bound packages called vesicles inside the hair cell to fuse with its surface membrane and release their contents to the outside. The contents, namely chemicals called neurotransmitters, then travels across the space between the cells, relaying the signal to the nerve cell.

The junctions between the hair cells and the nerve cells are more specifically known as ribbon synapses. This is because they have a ribbon-like structure that appears to tether a halo of vesicles close to the active zone where neurotransmitters are released. However, the exact role of this synaptic ribbon has remained mysterious despite decades of study.

The ribbon is mainly composed of a protein called Ribeye, and now Jean, Lopez de la Morena, Michanski, Jaime Tobón et al. show that mutant mice that lack this protein do not have any ribbons at their "ribbon synapses". Hair cells without synaptic ribbons are less able to timely and reliably send signals to the nerve cells, most likely because they cannot replenish the vesicles at the synapse quickly enough. Further analysis showed that the synaptic ribbon also helps to regulate the calcium channels at the synapse, which is important for linking the electrical signals in the hair cell to the release of the neurotransmitters.

Jean et al. also saw that hair cells without ribbons reorganize their synapses to form multiple active zones that could transfer neurotransmitter to the nerve cells. This could partially compensate for the loss of the ribbons, meaning the impact of their loss may have been underestimated. Future studies could explore this by eliminating the Ribeye protein only after the ribbon synapses are fully formed.

These findings may help scientists to better understand deafness and other hearing disorders in humans. They will also be of interest to neuroscientists who research synapses, hearing and other sensory processes.

DOl: https://doi.org/10.7554/eLife.29275.002

Synaptic ribbons of IHC afferent synapses were identified as presynaptic RIBEYE/CtBP2-immunofluorescent spots in wild-type (Figure 1B, RBE ${ }^{\mathrm{WT} / \mathrm{WT}}$ ) and heterozygous (Figure 1-figure supplement $1, \mathrm{RBE}^{\mathrm{WT} / \mathrm{KO}}$ ) mice. Their number per IHC did not change in the heterozygous condition (15.5 \pm 0.7 , S.D. $=1.58 ; n=50$ cells, $\mathrm{N}=4$ for RBE ${ }^{\mathrm{WT} / \mathrm{KO}}$ vs. $15.7 \pm 1.1$, S.D. $=2.19 ; n=39$ cells, $\mathrm{N}=3$ for $\mathrm{RBE}^{\mathrm{WT} / W T}$ at P21), while their intensity was significantly reduced (in arbitrary units: $3.4 \pm 0.7, \mathrm{~S}$. D. $=1.78 ; n=600$ spots for 40 cells, $N=3$ in $\mathrm{RBE}^{\mathrm{WT} / \mathrm{KO}}$ vs. $5.1 \pm 1.1$, S.D. $=2.23 ; n=411$ spots for 29 IHCs, N = 3 in RBE ${ }^{\mathrm{WT} / \mathrm{WT}}$; $\mathrm{p}<0.0001$, Mann-Whitney-Wilcoxon test; Figure 1-figure supplement $1 A, B) . R^{K B}{ }^{K O} / K O I H C$ s lacked synaptic RIBEYE/CtBP2 immunofluorescence spots (Figure 1B), while immunolabeling of nuclear CtBP2 remained present, corroborating previous findings in the retina (Maxeiner et al., 2016). The number of afferent synapses per IHC was determined by the count of postsynaptic densities (PSDs) identified as PSD-95 immunofluorescent spots (Figure 1C,D,E) and was unchanged when RIBEYE was removed $\left(13.7 \pm 0.8\right.$, S.D. $=2.04 ; n=56$ cells, $\mathrm{N}=4$ in $\mathrm{RBE}^{\mathrm{KO} / \mathrm{KO}}$ vs. $12.9 \pm 0.6$, S.D. $=2.13 ; n=55$ cells, $N=5$ in $\mathrm{RBE}^{\mathrm{WT} / \mathrm{WT}}$ ). Bassoon (Figure $1 \mathrm{C}$ ) and RIM2 (Figure 1D), both presynaptic scaffold proteins (Khimich et al., 2005; Jung et al., 2015a), remained present at the ribbonless afferent synapses of RBE ${ }^{\mathrm{KO} / \mathrm{KO}} \mathrm{IHCs}$ (marked by PSD-95). The scaffold protein piccolino, the short isoform of piccolo (Regus-Leidig et al., 2013) that is present in cochlear and retinal ribbons (Khimich et al., 2005; tom Dieck et al., 2005; Regus-Leidig et al., 2013), was absent from afferent synapses of RBE ${ }^{\mathrm{KO} / \mathrm{KO}} \mathrm{IHC}$. However, piccolo immunofluorescence was present in the vicinity of afferent synapses likely marking the long form piccolo at the efferent presynaptic AZs (Figure 1E, see schematic in Figure 1A). The PSD areas were calculated by fitting a 2 - 


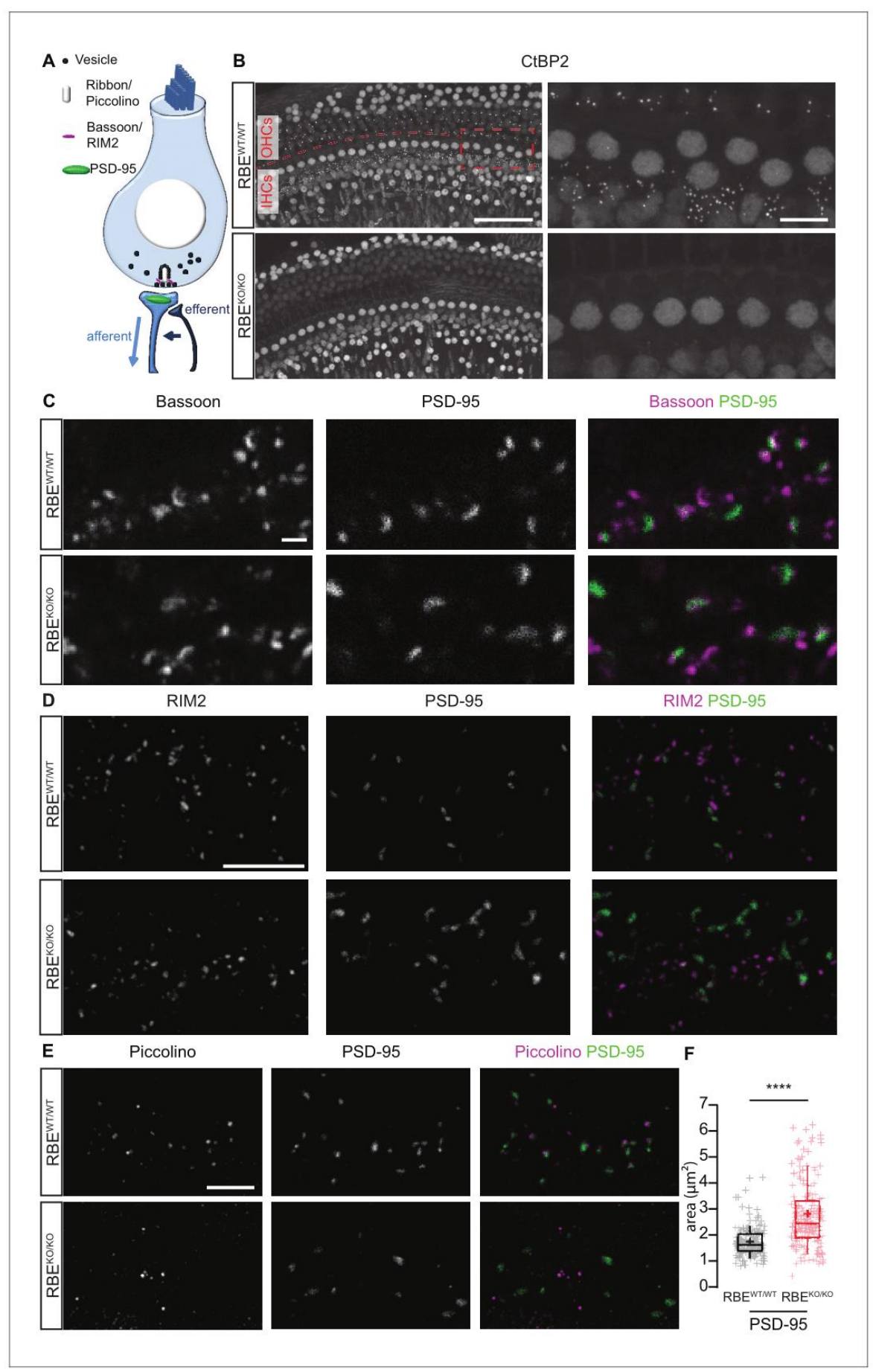

Figure 1. Loss of synaptic ribbons and piccolino from the AZs of RIBEYE-deficient IHCs. (A) Simplified schematic representation of an IHC with the afferent and efferent connectivities. (B) Maximal projection of confocal sections from organs of Corti immunolabeled for CtBP2 and RIBEYE, present in the nuclei and the ribbons, respectively. The RBE ${ }^{W T} / W^{T}$ staining (top row) shows small puncta in the outer hair cell (OHC) and IHC rows representing the synaptic ribbons, which are completely absent in the $\mathrm{RBE}^{\mathrm{KO} / \mathrm{KO}}$ hair cells (bottom row). Scale bar $=50 \mu \mathrm{m}$. Zoom into the IHC row (right column), Figure 1 continued on next page 
Figure 1 continued

emphasizes the complete disappearance of CtBP2-labeling at the basolateral part of RBE ${ }^{\mathrm{KO} / \mathrm{KO}}$ IHCs. Scale bar $=10 \mu \mathrm{m}$. (C) Maximal projection of confocal sections from organs of Corti co-labeled for the presynaptic marker and anchor of the ribbon, bassoon (left column), and the postsynaptic marker, PSD-95 (middle column), in RBE ${ }^{\mathrm{WT} / \mathrm{WT}}$ and RBE ${ }^{\mathrm{KO} / \mathrm{KO}} \mathrm{IHCs}$. The merged picture (right column) shows the juxtaposition of bassoon (magenta) with PSD-95 (green), indicating its presence both at RBE ${ }^{W T W T}$ and ribbonless RBE ${ }^{\mathrm{KO} / K O}{ }_{\mathrm{IHC}}$ synapses. Scale bar $=1 \mu \mathrm{m}$. (D) Maximal projection of confocal sections from organs of Corti co-labeled for the presynaptic marker RIM2 (left column) and the postsynaptic marker PSD-95 (middle column) The merged picture (right column) shows the co-localization of RIM2 (magenta) with PSD-95 (green) meaning its presence at the ribbonless IHC presynapses (scale bar $=5 \mu \mathrm{m}$ ). (E) Maximal projection of confocal sections from organs of Corti co-labeled for piccolino, a specific short splice variant of piccolo found at ribbons of RBE ${ }^{\text {WT }}$ WT IHC synapses (left column), co-labeled with PSD-95 (middle column). The merged pictures (right column) show PSD-95 (green) immunofluorescence lacking juxtaposed piccolino signal (magenta) in RBE ${ }^{\mathrm{KO} / K O}$ (bottom row), indicating absence of piccolino from afferent synapses of mutant IHCs. The punctate labeling for piccolo, away from PSD-95, most likely represents labeling of piccolo at conventional efferent synapses (schematically shown in Figure 1A). Scale bar $=5 \mu \mathrm{m}$. (F) Quantification of the area of PSD-95 immunofluorescent spots. The PSD-95 spots are siginificantly bigger in the RBE ${ }^{\mathrm{KO} / \mathrm{KO}}$ IHCs $\left(\mathrm{p}<0.0001\right.$, Mann-Whitney-Wilcoxon test, $n=178$ spots, $\mathrm{N}=3$ for RBE ${ }^{\mathrm{KO} / \mathrm{KO}}$ and $n=163 \mathrm{spots,}$ $\mathrm{N}=3$ for $\mathrm{RBE}^{\mathrm{WT} / \mathrm{WT}}$ ). Box plots show 10, 25, 50, 75 and $90^{\text {th }}$ percentiles with individual data points overlaid; means are shown as crosses.

DOl: https://doi.org/10.7554/eLife.29275.003

The following figure supplement is available for figure 1:

Figure supplement 1. Gene-dosage dependent expression of the RIBEYE at IHC AZs. DOl: https://doi.org/10.7554/eLife.29275.004

dimensional Gaussian function to each PSD-95 immunofluorescent spot, revealing a significant increase in the $\mathrm{RBE}^{\mathrm{KO} / \mathrm{KO}}$ condition $\left(2.82 \pm 0.09 \mu \mathrm{m}^{2}\right.$, S.D. $=1.25 ; n=178$ spots, $\mathrm{N}=3$ vs $1.74 \pm 0.05 \mu \mathrm{m}^{2}$, S.D. $=0.58 ; n=163$ spots, $\mathrm{N}=3$ in RBE ${ }^{\mathrm{WT} / W T}$ IHCs; $\mathrm{p}<0.0001$, Mann-Whitney-Wilcoxon; Figure 1F).

In order to study the effects of RIBEYE deletion on the ultrastructure of afferent IHC synapses, we performed transmission electron microscopy on random sections and electron tomography. Random ultrathin (70-75 nm) sections prepared from P21 mice (two animals per genotype) after aldehyde fixation and conventional embedding procedures showed that IHCs from $\mathrm{RBE}^{\mathrm{KO} / \mathrm{KO}}$ mice completely lack synaptic ribbons, while RBE ${ }^{\mathrm{WT} / \mathrm{WT}}$ and heterozygous RBE ${ }^{\mathrm{WT} / K O}$ typically display one ribbon per $A Z$ (Figure 2A-C). Interestingly, ribbons of RBE ${ }^{\mathrm{WT} / K O} \mathrm{IHC}$ were smaller in height, width and area compared to RBE ${ }^{\text {WT/WT }}$ IHC synaptic ribbons (Figure 2-figure supplement 1A-C; ribbon height: $118.32 \pm 3.17 \mathrm{~nm}$, S.D. $=31.84 \mathrm{~nm} ; n=101$ ribbons, $\mathrm{N}=2$ for RBE ${ }^{\mathrm{WT} / K O}$ vs. $197.09 \pm 4.36 \mathrm{~nm}, \mathrm{~S}$ D. $=44.93 \mathrm{~nm} ; n=106$ ribbons, $\mathrm{N}=2$ for $\mathrm{RBE}^{\mathrm{WT} / \mathrm{WT}}$; ribbon width: $119.80 \pm 6.23 \mathrm{~nm}, \mathrm{~S} . \mathrm{D} .=62.27$ $\mathrm{nm}$ for RBE ${ }^{\mathrm{WT} / K O}$ vs. $168.34 \pm 6.83 \mathrm{~nm}, \mathrm{~S} . \mathrm{D} .=70.27 \mathrm{~nm}$ for RBE ${ }^{\mathrm{WT}} / \mathrm{WT}$; ribbon area: $11.5 \mathrm{e} 3 \pm 6.2 \mathrm{e} 2$ $\mathrm{nm}^{2}$, S.D. $=6.3 \mathrm{e} 3 \mathrm{~nm}^{2}$ for RBE ${ }^{W T / K O}$ vs. $25.4 \mathrm{e} 3 \pm 1.1 \mathrm{e} 2 \mathrm{~nm}^{2}$, S.D. $=1.1 \mathrm{e} 3 \mathrm{~nm}^{2}$ for RBE fTT $^{W T}$; $p<0.0001$, Mann-Whitney-Wilcoxon test for all) agreeing with the significantly reduced ribbon immunofluorescence intensity in the $\mathrm{RBE}^{\mathrm{WT} / \mathrm{KO}}$ condition (see above and Figure 1-figure supplement 1A-B).

Random sections of synaptic contacts of $\mathrm{RBE}^{\mathrm{KO} / \mathrm{KO}}$ mice (Figure $2 \mathrm{C}$ ) often showed more than one presynaptic density (PD), each associated with a cluster of synaptic vesicles (henceforth considered individual AZs). The multiple AZs typically faced one continuous PSD, which is different from the synapses of immature IHC synapses that show multiple appositions of pre- and postsynaptic densities (Sendin et al., 2007; Wong et al., 2014). Moreover, we found more than one PD per synaptic contact in IHCs of older RBE ${ }^{\mathrm{KO} / \mathrm{KO}}$ mice (Figure 2E,F; 6 weeks and 8 months, respectively), arguing against a delayed synaptic maturation to be the cause of the phenotype. Sections from tangential cuts of the synapse (Figure 2D), reconstructions from serial ultrathin sections (Figure 2G, $G^{\prime}$ ) and quantifications of random sections (Figure $2 \mathrm{H}$ ) corroborated the notion of multiple small ribbonless $\mathrm{AZs}$ at the synaptic contacts of RBE ${ }^{\mathrm{KO} / \mathrm{KO}} \mathrm{IHCs}$. Analysis based on serial 3D reconstructions of synaptic contacts of RBE ${ }^{K O / K O}$ IHCs from P21 animals showed on average $1.92 \pm 0.34$ PDs (S.D. = 1.16; $n=17$ serial $3 D$ reconstructions, $N=2$ ) and $20.58 \pm 2.98$ total SVs per contact, S.D. $=10.34$ (Figure 2I). The lateral extent of the individual PDs, determined in random sections, was comparable between RBE ${ }^{\mathrm{KO} / \mathrm{KO}}$ and RBE ${ }^{\mathrm{WT} / \mathrm{WT}}$ synapses $(129.89 \pm 2.53 \mathrm{~nm}, \mathrm{~S} . \mathrm{D} .=26.26 \mathrm{~nm} ; \mathrm{n}=108 \mathrm{PDs}, \mathrm{N}=2$ for RBE $\mathrm{KO} / \mathrm{KO}$ vs. $129.35 \pm 4.89 \mathrm{~nm}, \mathrm{~S} . \mathrm{D} .=50.86 \mathrm{~nm} ; n=108 \mathrm{PDs}, \mathrm{N}=2$ for RBE $\mathrm{RT}^{\mathrm{W}} \mathrm{WT} ; \mathrm{p}=0.92$, $\mathrm{NPMC}$ test), while that of RBE ${ }^{\mathrm{WT} / \mathrm{KO}}$ was enlarged (Figure $2 M ; 157.64 \pm 7.19 \mathrm{~nm}$, S.D. $=72.24 \mathrm{~nm}$; $n=101$ PDs, $N=2 ; p=0.0004$ for comparison to RBEWT/WT, NPMC test). PSDs tended to be increased in length at RBE ${ }^{\mathrm{KO} / K O}$ synapses compared to RBE ${ }^{W T / W T}$ PSDs and were significantly larger 

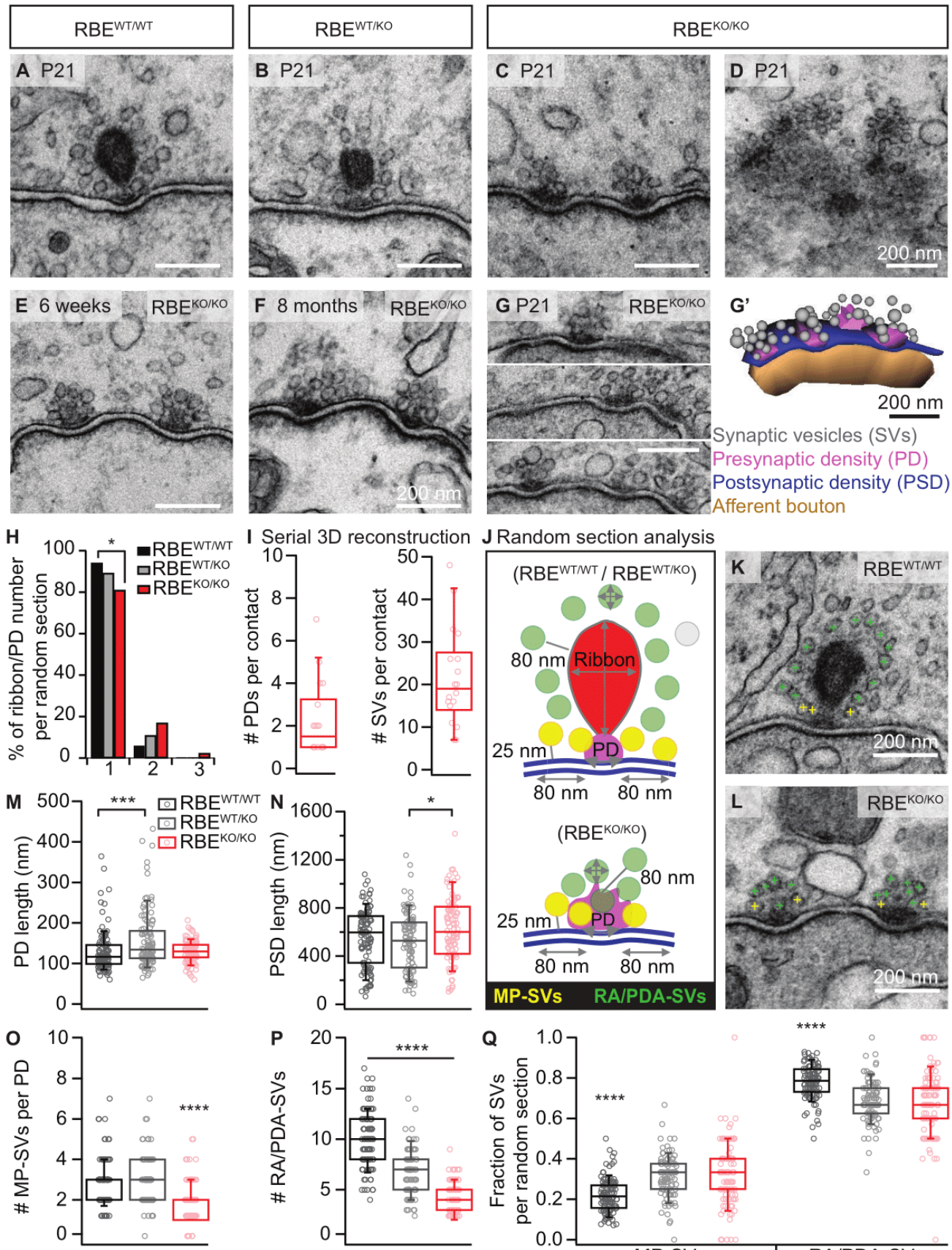

Figure 2. RIBEYE disruption transforms IHC synapses into contacts with multiple small ribbonless AZs. (A-C) Representative electron micrographs of IHC afferent synapses from P21 RBE ${ }^{W T / W T}, \mathrm{RBE}^{\mathrm{WT} / \mathrm{KO}}$ and RBE ${ }^{\mathrm{KO} / \mathrm{KO}}$ mice. Ribbonless $\mathrm{RBE}^{\mathrm{KO} / \mathrm{KO}}$ synapses display one or more presynaptic densities (PD) clustering SVs. Scale bars $=200 \mathrm{~nm}$. (D) RBE ${ }^{\mathrm{KO} / \mathrm{KO}} \mathrm{IHC}$ AZ cut tangentially, revealing multiple PDs (here six) per AZ. (E, F) Representative electron micrographs of RBE ${ }^{\mathrm{KO} / \mathrm{KO}} \mathrm{IHC}$ synapses from mice at 6 weeks $(\mathrm{E})$ and 8 months $(\mathrm{F})$ of age: the presence of at least $2 \mathrm{AZs}$ per contact in mature IHCs Figure 2 continued on next page 
Figure 2 continued

argues against a developmental delay. (G) Consecutive serial sections of a typical RBE ${ }^{\mathrm{KO} / \mathrm{KO}} \mathrm{P} 21 \mathrm{IHC}$ synapse showing multiple AZs. Scale bar $=200$ $\mathrm{nm}$. (G') Corresponding serial 3D reconstruction of the synapse in (G) showing four PDs (magenta) surrounded by a total of 48 SVs (gray). Scale bar $=$ $200 \mathrm{~nm}$. (H) Quantification of the number of ribbon/PD per random section. AZs with a single PD are less frequently observed in RBE ${ }^{\mathrm{KO} / K O}$ IHCs ( $n=108 \mathrm{AZs}, \mathrm{N}=2$ for RBE ${ }^{\mathrm{KO} / \mathrm{KO}}$ and $n=106 \mathrm{AZs}, \mathrm{N}=2$ for RBE ${ }^{\mathrm{WT} N \mathrm{NT}}$; $\mathrm{p}<0.05, \mathrm{NPMC}$ test). (I) Number of PDs and SVs per AZ in P21 RBE ${ }^{\mathrm{KO} / \mathrm{KO}}$ mice in serial $3 D$ reconstructions of $R B E^{K O / K O}$ afferent synapses. Box plots show 10, 25, 50, 75 and $90^{\text {th }}$ percentiles with individual data points overlaid, as for (M, N, O, P \& Q). (J) Schematic drawing illustrating the quantitative analysis of random sections. SV diameter: average of vertical and horizontal measurements from outer rim to outer rim. The ribbon height, width and area were measured as indicated by the gray lines. The length of the PD was determined along the AZ. For ribbon-occupied AZs: Membrane-proximal (MP) SVs (yellow) were counted in a distance of $\leq 25 \mathrm{~nm}$ from the AZ membrane (blue) and $\leq 80 \mathrm{~nm}$ from the PD. Ribbon-associated (RA) SVs were found in the first layer around the ribbon (red) with a maximum distance of $80 \mathrm{~nm}$ to the ribbon, quantified as indicated by the gray lines. For ribbonless AZs: Instead of RA-SVs we defined PD-associated SVs (PDA-SVs: all SV at PD with a maximum distance of $80 \mathrm{~nm}$ to the PD not matching the MP-SV criteria, defined as above). (K, L) Electron micrographs illustrating the quantification of the MP-SVs (yellow crosses) and the RA/PDA-SVs (green crosses). (M-Q) Quantification of random IHC synapse $\left(\mathrm{P}_{21}\right)$ sections revealed no significant differences between RBE ${ }^{K O} / K O$ and RBE ${ }^{W T} / W T$ for the PD and PSD length (PD length: $n=108 \mathrm{PDs}, \mathrm{N}=2 \mathrm{for}$ RBE $\mathrm{KO} / \mathrm{KO}$ and $n=108 \mathrm{PDs}$,

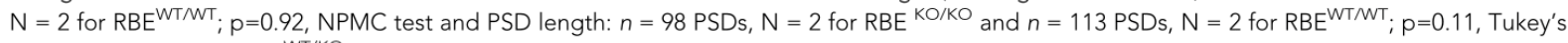
test). However, in the RBE ${ }^{W T / K O} I H C s$, the PDs were bigger than in the WT IHCs ( $n=101$ PDs, $N=2 ; p=0.0004$, NPMC test), and the PSDs were smaller than in the knock-out IHCs ( $n=100$ PSDs, $N=2$ for RBE ${ }^{W T / K O} ; p=0.01$, Tukey's test). MP-SVs $\left(n=108\right.$ AZs, N = 2 for RBE ${ }^{K O / K O}, n=106$ AZs, N = 2 for $\mathrm{RBE}^{\mathrm{WT} / W T} ; \mathrm{p}<0.0001, \mathrm{NPMC}$ test) and RA/PDA-SVs ( $n=108 \mathrm{AZs}, \mathrm{N}=2$ for RBE ${ }^{\mathrm{KO} / K O}, n=106 \mathrm{AZs}, \mathrm{N}=2$ for RBE RT/WT; $p<0.0001$, Tukey's test) per AZ, as well as the fraction of RA/PDA-SVs in RBE ${ }^{\mathrm{KO} / \mathrm{KO}}$, were significantly reduced ( $n=108 \mathrm{AZs}, \mathrm{N}=2$ for RBE ${ }^{\mathrm{KO} / \mathrm{KO}}, n=106 \mathrm{AZs}, \mathrm{N}=2$ for $\mathrm{RBE}{ }^{\mathrm{WT} / \mathrm{WT} \text {; }}$ $\mathrm{p}<0.0001, \mathrm{NPMC}$ test).

DOI: https://doi.org/10.7554/eLife.29275.005

The following figure supplement is available for figure 2:

Figure supplement 1. Random section analysis showed smaller synaptic ribbons and vesicles in $\mathrm{RBE}^{\mathrm{WT} / \mathrm{KO}}$ mice.

DOI: https://doi.org/10.7554/eLife.29275.006

than RBE ${ }^{\mathrm{WT} / \mathrm{KO}}$ PSDs (Figure $2 \mathrm{~N} ; 623.77 \pm 26.70 \mathrm{~nm}, \mathrm{~S} . \mathrm{D} .=264.33 \mathrm{~nm} ; \mathrm{n}=98 \mathrm{PSDs}, \mathrm{N}=2$ for $\mathrm{RBE}^{\mathrm{KO} / \mathrm{KO}}$ vs. $555.91 \pm 22.24 \mathrm{~nm}, \mathrm{~S} . \mathrm{D} .=236.42 \mathrm{~nm} ; n=113 \mathrm{PSDs}, \mathrm{N}=2$ for $\mathrm{RBE}^{\mathrm{WT} / \mathrm{WT}}$ vs. $521.34 \pm 24.20 \mathrm{~nm}$, S.D. $=242.03 \mathrm{~nm} ; n=100 \mathrm{PSDs}, \mathrm{N}=2$ for $\mathrm{RBE}^{\mathrm{WT} / \mathrm{KO}} ; \mathrm{p}=0.01$ for $\mathrm{RBE}^{\mathrm{KO} / \mathrm{KO}} \mathrm{vs}$. $\mathrm{RBE}^{\mathrm{WT} / K O}$, Tukey's test), which is consistent with the greater area of PSD-95 immunofluorescent spots in the knock-out condition (Figure 1E).

In the following, we characterized the populations of presynaptic SVs in random sections of vertically-cut IHC synapses. We counted membrane-proximal SVs (MP-SVs, $\leq 25 \mathrm{~nm}$ distance between SV membrane and plasma membrane, laterally within $80 \mathrm{~nm}$ of the PD, yellow in Figure $2 J-L$ ) as well as ribbon-associated SVs (RA-SVs, first layer of SVs around the ribbon within $80 \mathrm{~nm}$, green in Figure 2J, $K)$ or 'PD-associated' SVs (PDA-SVs, ribbonless AZs: SVs within $80 \mathrm{~nm}$ distance of the PD and not falling into the MP-SV pool (see above), green in Figure 2J,L). We found both MP-SVs (Figure 2O; $1.92 \pm 0.09$, S.D. $=0.93 ; n=108 \mathrm{AZs}, \mathrm{N}=2$ for $\mathrm{RBE}^{\mathrm{KO} / \mathrm{KO}}$ vs. $2.99 \pm 0.12, \mathrm{~S} . \mathrm{D} .=1.18 ; n=101 \mathrm{AZs}$ $\mathrm{N}=2$ for RBE ${ }^{\mathrm{WT} / \mathrm{KO}}$ vs. $2.77 \pm 0.12, \mathrm{~S} . \mathrm{D} .=1.18 ; n=106 \mathrm{AZs}, \mathrm{N}=2$ for $\mathrm{RBE}^{\mathrm{WT} / \mathrm{WT}} ; \mathrm{p}<0.0001$ for $\mathrm{RBE}^{\mathrm{KO} / \mathrm{KO}}$ vs. RBE ${ }^{\mathrm{WT} / W T}, \mathrm{NPMC}$ test) and PDA-SVs (Figure $2 \mathrm{P} ; 4.12 \pm 0.15, \mathrm{~S} . \mathrm{D} .=1.50 ; n=108 \mathrm{AZs}$, $\mathrm{N}=2$ for $\mathrm{RBE}^{\mathrm{KO} / \mathrm{KO}}$ vs. $10.09 \pm 0.27, \mathrm{~S} . \mathrm{D} .=2.75 ; n=106 \mathrm{AZs}, \mathrm{N}=2$ for $\mathrm{RBE}^{\mathrm{WT} / \mathrm{WT}} ; \mathrm{p}<0.0001$, Tukey's test) of the individual ribbonless IHC AZs of RBE ${ }^{K O / K O}$ mice to be significantly fewer than the corresponding number of MP-SVs and RA-SVs counted at RBE ${ }^{\text {WT } / W T}$ AZs. The fraction of PDA-SVs relative to the total number of SVs at RBE ${ }^{\mathrm{KO} / K O} \mathrm{AZs}$ was less than that of RA-SVs at RBE ${ }^{\mathrm{WT} / \mathrm{WT}} \mathrm{AZs}$ (Figure 2Q; $0.67 \pm 0.02$, S.D. $=0.16 ; n=108 \mathrm{AZs}, \mathrm{N}=2$ for $\mathrm{RBE}^{\mathrm{KO} / \mathrm{KO}}$ vs. $0.78 \pm 0.01$, S.D. $=0.08$; $n=106 \mathrm{AZs}, \mathrm{N}=2$ for RBE ${ }^{\mathrm{WT} / \mathrm{WT}} ; \mathrm{p}<0.0001, \mathrm{NPMC}$ test). Consequently, we observed an increase in the fraction of MP-SVs at RBE ${ }^{\mathrm{KO} / \mathrm{KO}} \mathrm{AZs}$ (Figure 2O; $0.33 \pm 0.02$, S.D. $=0.16 ; n=108 \mathrm{AZs}, \mathrm{N}=2$ for $\mathrm{RBE}^{\mathrm{KO} / \mathrm{KO}}$ vs. $0.22 \pm 0.01$, S.D. $=0.08 ; n=106 \mathrm{AZs}, \mathrm{N}=2$ for RBE ${ }^{\mathrm{WT} / \mathrm{WT}} ; \mathrm{p}<0.0001$, NPMC test). In line with the decreased ribbon size of RBE ${ }^{\mathrm{WT} / K O} A Z \mathrm{~s}$, we found a reduced number of RA-SVs, indicating a hypomorphic phenotype upon the loss of one allele of the RIBEYE gene. The SV diameter was unchanged for all three genotypes when jointly considering SVs of all categories in random sections (Figure 2-figure supplement 1D; $39.59 \pm 0.21 \mathrm{~nm}$, S.D. $=5.37 \mathrm{~nm} ; n=108 \mathrm{AZs}, \mathrm{N}=2$ for $\mathrm{RBE}^{\mathrm{KO} / \mathrm{KO}}$ vs. $40.53 \pm 0.14 \mathrm{~nm}, \mathrm{~S} . \mathrm{D} .=4.44 \mathrm{~nm} ; n=101 \mathrm{AZs}, \mathrm{N}=2$ for RBE ${ }^{\mathrm{WT} / \mathrm{KO}}$ vs. $41.80 \pm 0.13$ $\mathrm{nm}, \mathrm{S} . \mathrm{D} .=4.79 \mathrm{~nm} ; \mathrm{n}=106 \mathrm{AZs}, \mathrm{N}=2$ for RBE ${ }^{\mathrm{WT}} / \mathrm{WT} ; \mathrm{p}=0.30, \mathrm{NPMC}$ test). However, we found a subtle but significant SV-diameter reduction in $\mathrm{RBE}^{\mathrm{KO} / \mathrm{KO}}$ and $\mathrm{RBE}^{\mathrm{WT} / \mathrm{KO}}$ for MP-SVs (Figure 2-figure supplement $1 \mathrm{E} ; 39.29 \pm 0.34 \mathrm{~nm}, \mathrm{~S} . \mathrm{D}$. $=4.82 \mathrm{~nm} ; \mathrm{n}=108 \mathrm{AZs}, \mathrm{N}=2$ for $\mathrm{RBE}^{\mathrm{KO} / \mathrm{KO}} \mathrm{vs}$. $41.79 \pm 0.26 \mathrm{~nm}$, S.D. $=4.53 \mathrm{~nm} ; n=106 \mathrm{AZs}, \mathrm{N}=2$ for RBE ${ }^{\mathrm{WT} / \mathrm{WT}} ; \mathrm{p}<0.0001$, NPMC test and 
$40.29 \pm 0.25 \mathrm{~nm}, \mathrm{~S} . \mathrm{D} .=4.40 \mathrm{~nm} ; \mathrm{n}=101 \mathrm{AZs}, \mathrm{N}=2$ for $\mathrm{RBE}^{\mathrm{WT} / \mathrm{KO}}$ vs. RBE ${ }^{\mathrm{KO} / \mathrm{KO}} ; \mathrm{p}=0.03, \mathrm{NPMC}$ test) and for RA-/PDA-SVs (Figure 2-figure supplement 1F; $39.72 \pm 0.27 \mathrm{~nm}$, S.D. $=5.61 \mathrm{~nm}$ $n=108 \mathrm{AZs}, \mathrm{N}=2$ for RBE $\mathrm{KO} / \mathrm{KO}$ vs. $41.81 \pm 0.15 \mathrm{~nm}, \mathrm{~S} . \mathrm{D} .=4.86 \mathrm{~nm} ; n=106 \mathrm{AZs}, \mathrm{N}=2$ for $\mathrm{RBE}^{\mathrm{WT} / \mathrm{WT}} ; \mathrm{p}<0.0001, \mathrm{NPMC}$ test and $\mathrm{RBE}^{\mathrm{KO} / \mathrm{KO}}$ vs. $40.63 \pm 0.17 \mathrm{~nm}$, S.D. $=4.45 \mathrm{~nm} ; n=101 \mathrm{AZs}$, $\mathrm{N}=2$ for RBE ${ }^{\mathrm{WT} / \mathrm{KO}} ; \mathrm{p}=0.003, \mathrm{NPMC}$ test and $\mathrm{RBE}^{\mathrm{WT} / \mathrm{KO}} \mathrm{vs}$. RBE ${ }^{\mathrm{WT} / \mathrm{WT}} ; \mathrm{p}=0.02, \mathrm{NPMC}$ test).

Next, to capture the synapses in a near-to-native state and to evaluate vesicle tethering, we performed electron tomography on $250 \mathrm{~nm}$ thick sections that were prepared with high-pressure freezing and freeze-substitution (HPF/FS) of organs of Corti from P21 mice (Figure 3). Tomography confirmed the absence of synaptic ribbons and the presence of multiple AZs per contact, each with a clear PD (Figure 3B,D,F). However, we note that the $250 \mathrm{~nm}$ thick sections did typically not fully cover the synaptic contact, which leads to an underestimation for the total number of SVs particularly for the spatially extended $\mathrm{RBE}^{\mathrm{KO} / \mathrm{KO}}$ synapses. The PDs appeared roundish in the $\mathrm{RBE}^{\mathrm{KO} / \mathrm{KO}}$ with MP-SVs closely arranged around the PD as found at the more elongated RBE ${ }^{\mathrm{WT} / \mathrm{WT}}$ AZs (Figure $3 C$, $D$ ). For the tomograms, we followed the definition of MP-SV pool according to the 2D-random sections (Figure 2), but in addition we measured the MP-SVs also in a maximum distance of $50 \mathrm{~nm}$ from the $\mathrm{AZ}$ membrane and $\leq 100 \mathrm{~nm}$ from the PD (Figure 3-figure supplement 1 and supplementary file 1). This was motivated by the presence of long tethers connecting SV and AZ membrane and was previously introduced (Jung et al., 2015a). Further, we distinguished between tethered and non-tethered SVs (Figure 3G, Figure 3-figure supplement 1A). There, we focused our analysis on tethers to the ribbon/PD, plasma membrane and those interconnecting two adjacent $\mathrm{SVs}$ (Figure $3 \mathrm{H}, I, M, N$ ). We found a significant reduction in the number of MP-SVs per AZ in RBE ${ }^{\mathrm{KO}}$ / $\mathrm{KO}$ IHCs (Figure 3J; RBE ${ }^{\mathrm{KO} / \mathrm{KO}}=6.30 \pm 0.86$, S.D. $=2.87 \mathrm{MP}-\mathrm{SVs} ; n=11 \mathrm{AZs}, \mathrm{N}=3$ vs. RBE ${ }^{\mathrm{WT} / \mathrm{WT}}=$ $8.70 \pm 0.82$, S.D. $=2.45 \mathrm{MP}-\mathrm{SVs} ; n=9 \mathrm{AZs}, \mathrm{N}=3 ; \mathrm{p}=0.04$, Mann-Whitney-Wilcoxon test; significant also by the second analysis method: Figure 3-figure supplement $1 B$, supplementary file 1 ), while the fraction of tethered MP-SVs (No. of tethered MP-SVs/No. of all MP-SVs) was not significantly altered (Figure $3 K ; \mathrm{RBE}^{\mathrm{KO} / \mathrm{KO}}=0.75 \pm 0.07$, S.D. $=0.24 ; n=11 \mathrm{AZs}, \mathrm{N}=3 \mathrm{vs}$. RBE ${ }^{\mathrm{WT} / \mathrm{WT}}=0.65 \pm$ $0.06, \mathrm{~S} . \mathrm{D} .=0.18 ; n=9 \mathrm{AZs}, \mathrm{N}=3 ; \mathrm{p}=0.30$, t-test; Figure 3-figure supplement $1 C$, supplementary file 1). The majority of the MP-SVs were tethered via a single tether in both RBE ${ }^{\mathrm{WT}}$ / $W T$ and $R B E^{K O / K O} I H C s$. The fraction of MP-SVs with multiple $(\geq 2)$ tethers was significantly larger in $\mathrm{RBE}^{\mathrm{KO} / \mathrm{KO}}$ IHCs (Figure 3K; single-tethered MP-SVs: $\mathrm{RBE}^{\mathrm{KO} / \mathrm{KO}}=0.55 \pm 0.06, \mathrm{~S} . \mathrm{D} .=0.19 ; n=11$ $\mathrm{AZs}, \mathrm{N}=3$ vs. $\mathrm{RBE}^{\mathrm{WT} / \mathrm{WT}}=0.61 \pm 0.06$, S.D. $=0.17 ; n=9 \mathrm{AZs}, \mathrm{N}=3 ; \mathrm{p}=0.81 ;$ multiple-tethered MP-SVs: RBE ${ }^{\mathrm{KO} / \mathrm{KO}}=0.20 \pm 0.05, \mathrm{~S} . \mathrm{D} .=0.15 ; n=11 \mathrm{AZs}, \mathrm{N}=3 \mathrm{vs} . \mathrm{RBE}^{\mathrm{WT} / \mathrm{WT}}=0.04 \pm 0.02, \mathrm{~S}$. D. $=0.05 ; n=9 \mathrm{AZs}, \mathrm{N}=3 ; \mathrm{p}=0.01$, Tukey's test; Figure 3-figure supplement $1 \mathrm{C}$ supplementary file 1). Further, and in line with analysis of random sections, the number of PDA-SVs per $\mathrm{RBE}^{\mathrm{KO} / \mathrm{KO}} \mathrm{AZ}$ was smaller than that of RA-SVs at RBE ${ }^{\mathrm{WT} / W T}$ AZs (Figure 3O; RBE ${ }^{\mathrm{KO} / \mathrm{KO}}$ : $9.30 \pm 1.13$, S.D. $=3.74$ PDA-SVs; $n=11 \mathrm{AZs}, \mathrm{N}=3 \mathrm{vs} . \mathrm{RBE}^{\mathrm{WT} / \mathrm{WT}}: 30.33 \pm 3.00$, S.D. $=9.01 \mathrm{RA}-\mathrm{SV}$; $n=9 \mathrm{AZs}, \mathrm{N}=3 ; \mathrm{p}<0.0001$, Mann-Whitney-Wilcoxon test; Figure 3-figure supplement 1 , supplementary file 1). However, the fraction of PDA-SVs tethered to the PD was not different from that of RA-SVs tethered to the ribbon (Figure 3P; RBE ${ }^{\mathrm{KO} / \mathrm{KO}}: 0.80 \pm 0.06$, S.D. $=0.19$ tethered PDASV fraction; $n=11 \mathrm{AZs}, \mathrm{N}=3 \mathrm{vs}$. RBE ${ }^{\mathrm{WT} / \mathrm{WT}}: 0.70 \pm 0.06, \mathrm{~S} . \mathrm{D} .=0.17$ tethered RA-SV fraction; $n=9$ $A Z s, N=3 ; p=0.12$, t-test; Figure 3-figure supplement 1F, supplementary file 1).

Finally, tomography indicated unchanged SV diameters at $\mathrm{RBE}^{\mathrm{KO} / \mathrm{KO}} \mathrm{AZs}$ (Figure 3L, Q; MP-SV diameter: $50.17 \pm 0.90 \mathrm{~nm}, \mathrm{~S}$.D. $=2.95 \mathrm{~nm} ; n=11 \mathrm{PDs}, \mathrm{N}=3$ for RBE ${ }^{\mathrm{KO} / \mathrm{KO}}$ vs. $47.81 \pm 0.60 \mathrm{~nm}$, S. D. $=1.70 ; n=9$ ribbons, $\mathrm{N}=3$ for RBE ${ }^{\mathrm{WT} / \mathrm{WT}} ; \mathrm{p}=0.06$, Mann-Whitney-Wilcoxon test, Figure 3-figure supplement 1D, supplementary file 1; RA/RA-SV diameter: $49.71 \pm 0.83 \mathrm{~nm}$, S.D. $=2.75$ $n=11$ PDs, $N=3$ for RBE ${ }^{\mathrm{KO} / \mathrm{KO}}$ vs. $49.80 \pm 0.78 \mathrm{~nm}, \mathrm{~S} . \mathrm{D} .=2.35 ; n=9$ ribbons, $\mathrm{N}=3$ for RBE ${ }^{\mathrm{WT} / \mathrm{WT}}$; $\mathrm{p}=0.71$, Mann-Whitney-Wilcoxon test; Figure 3-figure supplement 1G, supplementary file 1). We presume that differences in the comparison of $\mathrm{RBE}^{\mathrm{KO} / \mathrm{KO}}$ and RBE ${ }^{\mathrm{WT} / \mathrm{WT}}$ between the random section and electron tomography analysis primarily reflects the larger number of $A Z$ analyzed by the former approach.

We then used confocal and stimulated emission depletion (STED) super-resolution immunofluorescence microscopy in order to study the abundance and spatial organization of presynaptic $\mathrm{Ca}_{\mathrm{v}} 1.3$ $\mathrm{Ca}^{2+}$-channels (Neef et al., 2018), which contribute more than $90 \%$ of the voltage-gated $\mathrm{Ca}^{2+}$-influx into IHCs (Platzer et al., 2000; Brandt et al., 2003; Dou et al., 2004). Organs of Corti from 3week-old $\mathrm{RBE}^{\mathrm{KO} / \mathrm{KO}}$ and $\mathrm{RBE} \mathrm{WT}^{\mathrm{W}} \mathrm{WT}$ mice were processed in parallel for immunohistochemistry and imaging. Cav1.3 $\mathrm{Ca}^{2+}$-channels remained clustered at $\mathrm{RBE}^{\mathrm{KO} / \mathrm{KO}} \mathrm{AZs}$ and were identified as $\mathrm{Cav} 1.3$ 


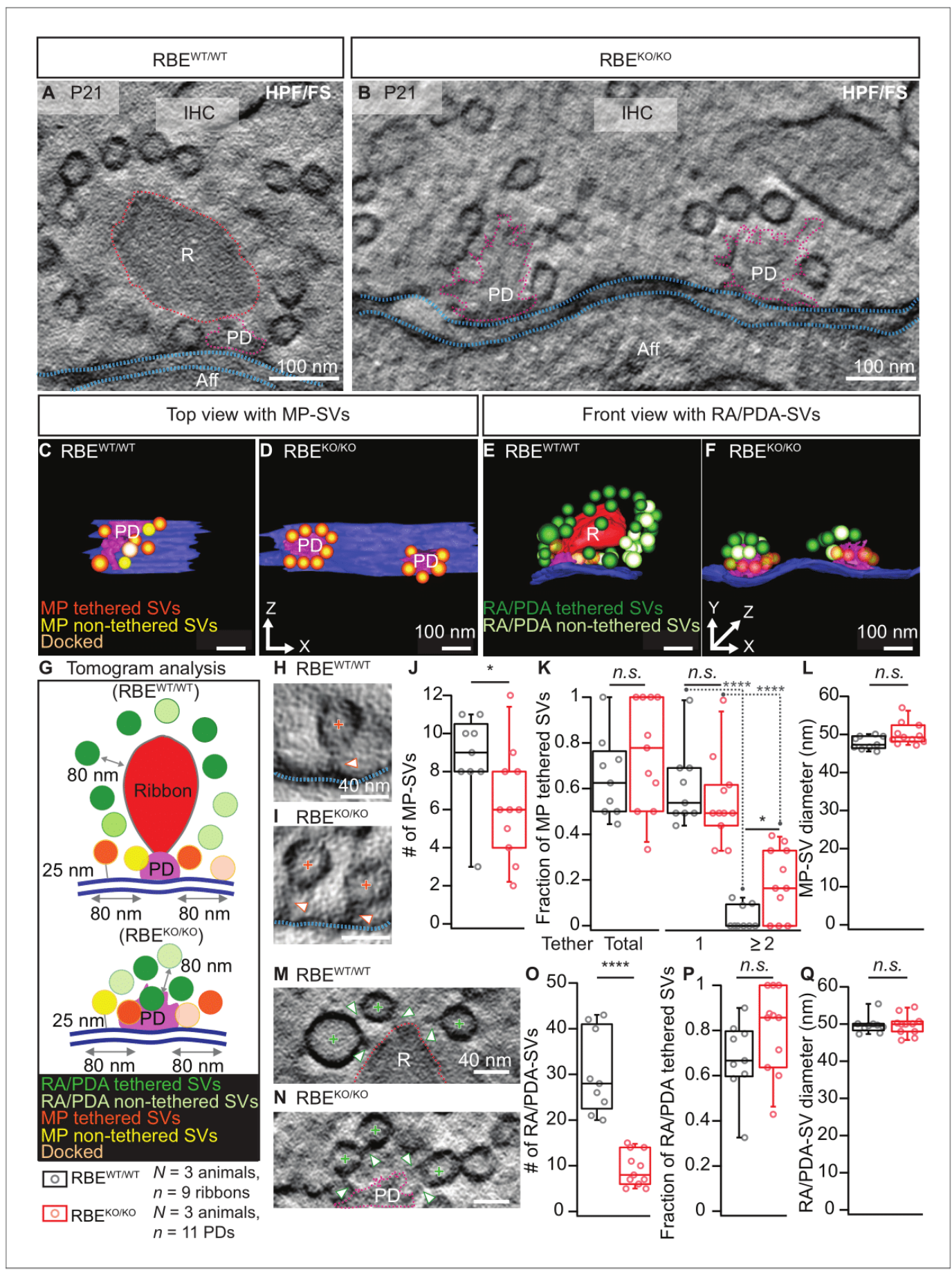

Figure 3. Electron tomography analysis of synaptic ultrastructure obtained after HPF/FS. (A, B) Exemplary virtual electron tomographic sections of P21 $\mathrm{RBE}^{\mathrm{WT} M T}(\mathrm{~A})$ and $\mathrm{RBE}{ }^{\mathrm{KO} / \mathrm{KO}}$ (B) highlight the ribbon $\mathrm{R}$ in red, the presynaptic density $(\mathrm{PD})$ in magenta and the AZ membrane with blue dotted lines. No synaptic ribbons, but two PDs were observed in $\mathrm{RBE}^{\mathrm{KO} / \mathrm{KO}}(\mathrm{B})$. Scale bars $=100 \mathrm{~nm}$. (C-F) $3 \mathrm{D}$ rendered models of $\mathrm{RBE}^{\mathrm{WT}} \mathrm{WT}(\mathrm{C}, \mathrm{E})$ and $\mathrm{RBE} \mathrm{K}^{\mathrm{KO} / \mathrm{KO}}(\mathrm{D}, \mathrm{F})$ IHC synapses. (C, D) The top view depicts the MP-SV pool with tethered (orange), non-tethered (yellow) and docked (light orange) SVs. For clarity Figure 3 continued on next page 
Figure 3 continued

ribbons, RA/PDA-SVs are removed. Scale bars $=100 \mathrm{~nm}$. (E,F) The front view shows the RA/PDA-SV pool from RBE ${ }^{W T / W T}(E)$ and RBE ${ }^{\mathrm{KO} / K O}(\mathrm{~F})$ IHCs. Tethered (dark green) and non-tethered (light green) RA/PDA-SVs. For the ease of visualization, the MP-SV pool is transparent here and other synaptic structures such as ribbon (red), PD (magenta) and AZ membrane (blue) are indicated. Scale bars $=100 \mathrm{~nm}$. (G) Illustrations show the tomogram analysis parameters comparable to 2D-random section analysis (Figure 2), in addition to that the vesicle pools are subdivided into tethered, non-tethered and docked SVs. (H, I, M, N) Representative tomogram virtual sections of membrane-tethered MP-SVs ( $\mathrm{H}$, l; orange cross), ribbon/PD tethered SVs and ribbon/PD proximal interconnecting SVs $\left(M, N\right.$; green cross) in $\operatorname{RBE}^{W T} / \mathrm{NT}(\mathrm{H}, \mathrm{M})$ and in $\operatorname{RBE}^{\mathrm{KO} / \mathrm{KO}}(\mathrm{I}, \mathrm{N})$. Tethers are marked with a white arrowhead and other synaptic entities are color-coded similar to (A, B). Scale bars $=40 \mathrm{~nm}$. (J-L) Quantification for the MP-SV pool is depicted; $n=9$ ribbons, N $=3$ animals for RBE ${ }^{W T} / W T$ and $n=11$ PDs, $N=3$ animals for RBE ${ }^{K O / K O}$. Fewer MP-SV were observed in RBE ${ }^{K O} / K O$ (J; $p=0.04, M a n n-W h i t n e y-W i l c o x o n$ test). The fraction of tethered MP-SVs was unaltered in $\mathrm{RBE}^{\mathrm{KO} / \mathrm{KO}}\left(\mathrm{K} ; \mathrm{p}=0.30\right.$, t-test). Most of the SVs were tethered by a single tether in both RBE ${ }^{\mathrm{KO} / K O}$ and $\mathrm{RBE}^{\text {WT }}$ WT . Significantly more SVs with multiple-tethers were observed in RBE ${ }^{\mathrm{KO} / \mathrm{KO}}$ (K; single tethered MP-SVs, multiple-tethered MP-SVs: n.s.: p>0.05, * $p=0.01$, ${ }^{\star * * *}: p<0.0001$, Tukey's test). MP-SV diameter was unaltered in RBE ${ }^{K O} / K O$ (L; $p=0.06$, Mann-Whitney-Wilcoxon test). (O-Q) Quantification for the RA/PDA-SVs, sample size is same as for the MP-SV analysis. Significantly fewer PDA-SVs were observed in RBE ${ }^{\mathrm{KO} / K O}\left(\mathrm{O} ; \mathrm{RBE}^{\mathrm{KO} / \mathrm{KO}}\right.$ : $\mathrm{P}<0.0001, \mathrm{Mann}$ Whitney-Wilcoxon test). The fraction of PDA tethered SVs in RBE ${ }^{\mathrm{KO} / K O}$ was comparable to RA tethered SVs in RBE ${ }^{\mathrm{WT} / \mathrm{WT}}$ (P; $\mathrm{p}=0.12, \mathrm{t}$-test). SV diameters were unaltered in the RA/PDA vesicle pool ( $Q ; p=0.06$, Mann-Whitney-Wilcoxon test). Box plots show 10, 25, 50, 75 and $90^{\text {th }}$ percentiles with individual data points overlaid. See Figure 3-figure supplement 1 and supplementary file 1 for modified tomogram analysis according to Jung et al., 2015 a. DOl: https://doi.org/10.7554/eLife.29275.007

The following figure supplement is available for figure 3 :

Figure supplement 1. Electron tomogram analysis according to Jung et al., 2015a. DOI: https://doi.org/10.7554/eLife.29275.008

labeling juxtaposed to PSD-95 immunofluorescent spots (Figure 4A). In order to analyze the spatial organization of synaptic $\mathrm{Ca}^{2+}$-channels, we performed 3-color, 2D-STED immunofluorescence imag-

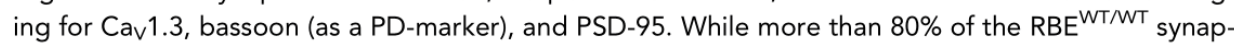
ses showed the typical stripe-like co-alignment of Cav1.3 and bassoon immunofluorescence (Neef et al., 2018), imaging of $\mathrm{RBE}^{\mathrm{KO} / \mathrm{KO}}$ synapses indicated a high prevalence (over $70 \%$ ) of smaller, rounder and often several $\mathrm{Ca}^{2+}$-channel clusters and PDs per synaptic contact (Figure 4B, C). We then quantified stripe-like clusters by measuring their long and short axis using 2D Gaussian fits and found no differences between $\mathrm{RBE}^{\mathrm{KO} / \mathrm{KO}}$ and $\mathrm{RBE}^{\mathrm{WT} / \mathrm{WT}}$ AZs (Figure 4D). Finally, we quantified the number of Cav1.3-immunofluorescent structures per contact (as indicated by PSD-95 immunofluorescence). While more than $80 \%$ of RBE ${ }^{\text {WT/WT }}$ synapses displayed a single cluster, over $60 \%$ of the $\mathrm{RBE}^{\mathrm{KO} / \mathrm{KO}}$ synapses contained two or more $\mathrm{Ca}^{2+}$-channel clusters (Figure 4E). Hence, the average number of Cav1.3-immunofluorescent structures was significantly higher at $\mathrm{RBE}^{\mathrm{KO} / \mathrm{KO}}$ synapses compared to RBE ${ }^{\text {WT/WT }}(2.06 \pm 0.09$, S.D. $=1.16 ; n=178$ spots, $\mathrm{N}=3$ vs. $1.16 \pm 0.03$, S.D. = 0.38; $n=183$ spots, $N=2 ; p<0.0001$, Mann-Whitney-Wilcoxon test) and we likely underestimated this difference due to the low resolution of 2D-STED in the z-axis. In summary, our results indicate that RIBEYE-disruption transforms the single ribbon-type $A Z$ into a complex presynaptic organization with multiple conventional-like AZs facing the postsynaptic bouton.

\section{Altered operating range of presynaptic $\mathrm{Ca}^{2+}$-influx at ribbonless IHC synapses}

Next, we combined whole-cell patch-clamp with confocal $\mathrm{Ca}^{2+}$-imaging of IHCs to study $\mathrm{Ca}^{2+}$-influx at the whole $\mathrm{IHC}$ and single synapse levels using $5 \mathrm{mM}\left[\mathrm{Ca}^{2+}\right]_{\mathrm{e}}$ to augment the signal to noise. Using step-depolarizations in conditions that isolated the $\mathrm{Ca}^{2+}$-current (see Materials and methods), we probed the amplitude and voltage-dependence of $\mathrm{IHC} \mathrm{Ca}{ }^{2+}$-influx (Figure 5A). The amplitude of $\mathrm{Ca}^{2+}$-influx (Figure $5 \mathrm{~A}_{i}$; for $\mathrm{Ca}^{2+}$-current density, see Figure $5 \mathrm{~A}_{i i}$ ) was unaltered in $\mathrm{RBE}^{\mathrm{KO} / \mathrm{KO}} \mathrm{IHCs}_{\mathrm{H}}(-$ $151 \pm 12.9 \mathrm{pA}, \mathrm{S} . \mathrm{D} .=59 \mathrm{pA} ; n=21 \mathrm{IHCs}, \mathrm{N}=8$ in $\mathrm{RBE}^{\mathrm{KO} / \mathrm{KO}}$ vs. $-161 \pm 15.4 \mathrm{pA}, \mathrm{S} . \mathrm{D} .=71 \mathrm{pA} ;$ $n=21 \mathrm{IHCs}, \mathrm{N}=9$ in RBE ${ }^{\mathrm{WT} / \mathrm{WT}} ; \mathrm{p}=0.62$, t-test), in agreement with findings in retinal bipolar neurons (Maxeiner et al., 2016) but in contrast to our previous findings in ribbon-deficient IHCs of bassoon mutant mice (Khimich et al., 2005; Frank et al., 2010; Jing et al., 2013). Kinetics of $\mathrm{Ca}^{2+}$-channel activation were unchanged (Figure 5B), whereas inactivation kinetics were slightly faster in the $\mathrm{RBE}^{\mathrm{KO} / \mathrm{KO}} \mathrm{IHCs}$ (smaller residual $\mathrm{Ca}^{2+}$-current at $200 \mathrm{~ms}$ of depolarization (normalized to the peak current): $0.82 \pm 0.007$, S.D. $=0.02 ; n=10 \mathrm{lHCs}, \mathrm{N}=5$ for RBE ${ }^{\mathrm{KO} / \mathrm{KO}}$ vs. $0.85 \pm 0.01$, S.D. $=0.04$; $n=11 \mathrm{IHCs}, \mathrm{N}=7$, in the RBE ${ }^{\mathrm{WT} / \mathrm{WT}}$ condition; $\mathrm{p}=0.017$, Mann-Whitney-Wilcoxon test; Figure 5D). When analyzing the voltage-dependence of $\mathrm{Ca}^{2+}$-channel activation (Figure $5 \mathrm{C}$ ), we found a small (2 
eLIFE Research article
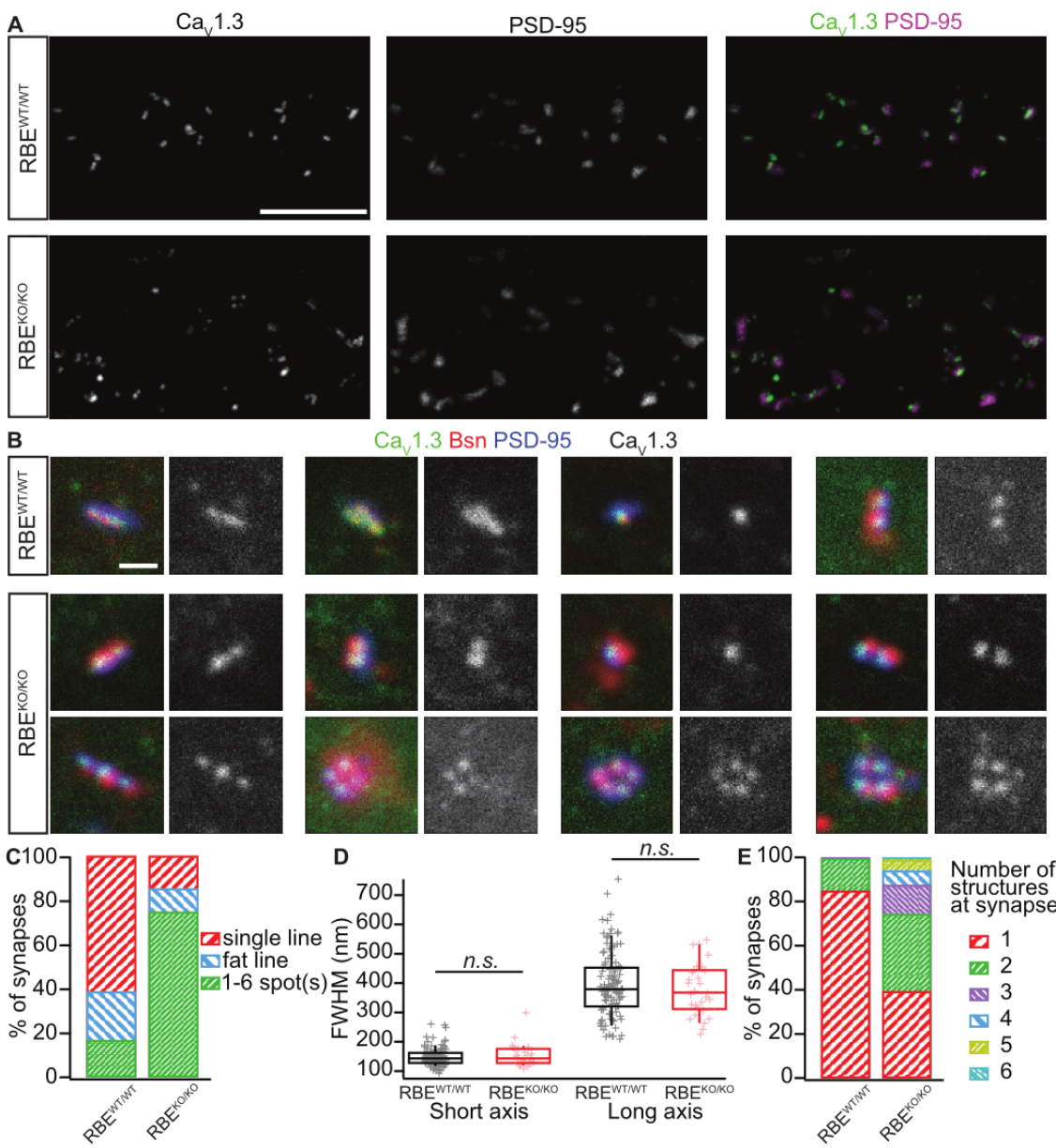

D
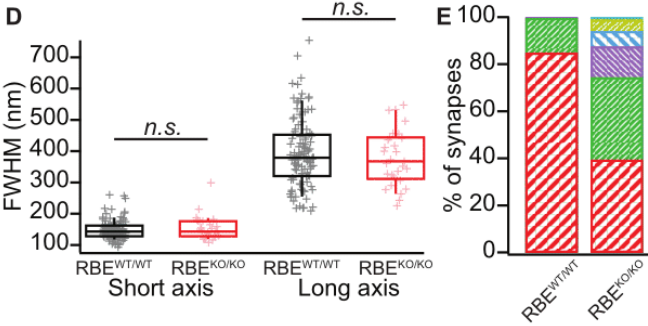

Number of structures
at synapse

口 1

प 2

四 3

$\square 4$

ㄴ. 5

Figure 4. Analyzing AZ $\mathrm{Ca}^{2+}$-channel clusters using confocal and STED immunofluorescence microscopy. (A) Maximal projections of confocal sections from organs of Corti immunolabeled for Cav1.3 $\mathrm{Ca}^{2+}$-channels (left column) and PSD-95 (middle column). The merged pictures (right column) show their juxtaposition in both conditions. While we mostly found one linear/spot-like $\mathrm{Ca}^{2+}$-channel cluster per PSD in RBE ${ }^{\mathrm{WT}}$ WT IHCs (top row), we often observed several spots per PSD in RBE ${ }^{\mathrm{KO} / K O}{ }_{\mathrm{IHCs}}$ (bottom row). Scale bar $=5 \mu \mathrm{m}$. (B) Triple co-labeling of Cav1.3 (green), bassoon (red), and PSD-95 (blue) at several IHC AZs from RBE ${ }^{\mathrm{WT} / W T}$ and RBE ${ }^{\mathrm{KO} / K O}$ mice, imaged in 2D-STED (Cav1.3 and PSD-95) and confocal mode (bassoon), showing that Cav1.3 $\mathrm{Ca}^{2+}$-channels cluster at AZs in IHCs of both genotypes. Cav1.3 immunofluorescence is displayed in gray next to the merged image for better visualization. Scale bar $=500 \mathrm{~nm}$. (C) $178 \mathrm{RBE}^{\mathrm{WT} / \mathrm{WT}}$ and $183 \mathrm{RBE}^{\mathrm{KO} / \mathrm{KO}}$ synapses were categorized according to the pattern of Cav1.3 immunofluorescence found by assigning them to a group of either line-shaped clusters, fat line-shaped clusters or one/multiple spots. A markedly higher fraction of synapses was found to display a spot-like Cav1.3-signal in RBE ${ }^{\mathrm{KO} / K O}$ than in RBEWT/WT IHCs. (D) Fitting of a 2D-Gaussian function to the immunofluorescence data of the line-shaped Cav1.3 clusters showed no difference in terms of size between RBE ${ }^{\text {WT WT }}$ and RBE ${ }^{\mathrm{KO} / \mathrm{KO}}$ clusters, as estimated by the full width at half maximum of the Gaussian's short and long axis. Box plots show 10, 25, 50, 75 and $90^{\text {th }}$ percentiles with individual data points overlaid. (E) Quantification of the number of fluorescent structures (lines or spots) labeled by the anti-Cav1.3 antibody at RBE ${ }^{W T / W T}$ and $\mathrm{RBE}^{\mathrm{KO} / \mathrm{KO}}$ synapses showed a significantly increased number in the knockout $(\mathrm{p}<0.0001$, Mann-Whitney-Wilcoxon test).

DOI: https://doi.org/10.7554/eLife.29275.009

$\mathrm{mV}$ ) but significant depolarizing shift of the potential of half-maximal $\mathrm{Ca}^{2+}$-channel activation, $\mathrm{V}_{\mathrm{h}}$ (Figure $5 C_{i},-22.96 \pm 0.43 \mathrm{mV}, \mathrm{S} . \mathrm{D} .=2.39 \mathrm{mV} ; n=21 \mathrm{IHCs}, \mathrm{N}=8$ in $\mathrm{RBE}^{\mathrm{KO} / \mathrm{KO}}$ vs. $-25.04 \pm 0.65$ $\mathrm{mV}, \mathrm{S} . \mathrm{D} .=2.98 \mathrm{mV} ; n=21 \mathrm{HCs}, \mathrm{N}=9 \mathrm{in} \mathrm{RBE}^{\mathrm{WT} / \mathrm{WT}} ; \mathrm{p}=0.017$, t-test). When analyzed in a smaller data set recorded in $2 \mathrm{mM}\left[\mathrm{Ca}^{2+}\right]_{\mathrm{e}}$ the depolarized $\mathrm{V}_{\mathrm{h}}$-shift did not reach statistical significance (data 


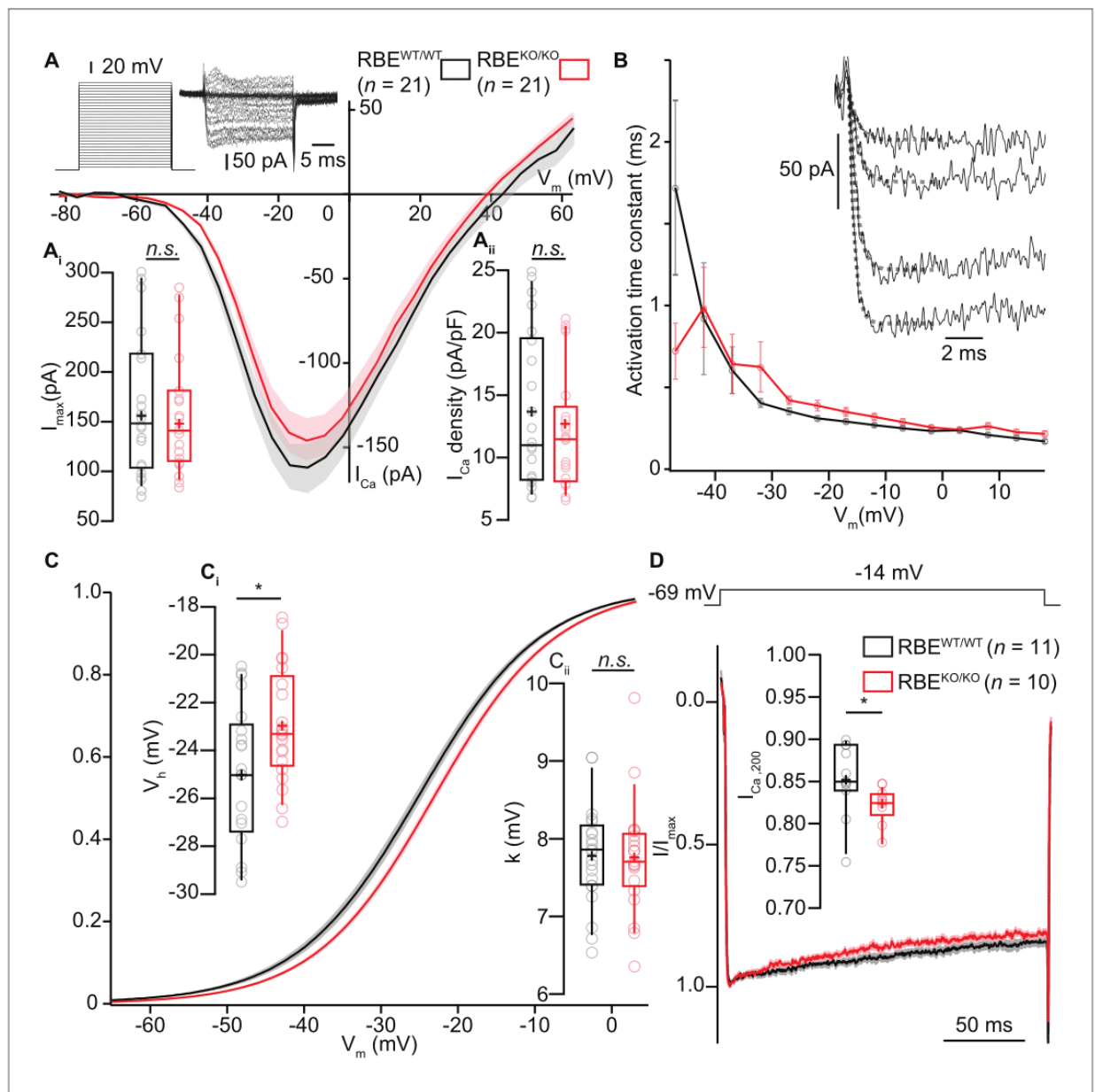

Figure 5. IHC Ca ${ }^{2+}$-influx shows normal amplitude but a mild depolarized shift in voltage-dependence. (A) IV-relationship of the whole-cell $\mathrm{Ca}^{2+}$. current in RBE ${ }^{W T W T}$ (black, $n=21$ cells, $N=8$ ) and RBE KO/KO (red, $n=21$ cells, $N=8$ ) IHCs show comparable (sign-inverted) current amplitudes ( $\mathrm{A}_{\mathrm{i}}$ $\mathrm{p}=0.62$, t-test) and density $\left(\mathbf{A}_{\mathrm{ii}}, \mathrm{p}=0.37\right.$, t-test.). Mean (line) \pm S.E.M. (shaded areas) are displayed as for (C, $\left.\mathbf{D}\right)$. The protocol, consisting of $20 \mathrm{~ms}$ steps of $5 \mathrm{mV}$ from -82 to $+63 \mathrm{mV}$, as well as exemplary resulting currents, aredisplayed in the left. Box plots show 10, 25, 50, 75 and $90^{\text {th }}$ percentiles with individual data points overlaid, means are shown as crosses, as for (C, D). (B) Activation time constants (mean \pm S.E.M.) of Ca ${ }^{2+}$-currents at different potentials were obtained by fitting a power exponential equation to the first $5 \mathrm{~ms}$ of the current traces, revealing no differences between conditions. (C) Fractional activation of the whole-cell $\mathrm{Ca}^{2+}$-current derived from the IV-relationships (A) was fitted to a Boltzmann function. (Ci) Box plots of the voltage for half-maximal activation $V_{h}$ and $V_{h}$-estimates of individual IHCs show a depolarized shift of the fractional activation of the Cav $1.3 C^{2+}$-channels in the $\mathrm{RBE}^{\mathrm{KO} / \mathrm{KO}}$ IHCs ( $\mathrm{p}=0.029$, t-test). (Cii) Box plots of the voltage-sensitivity or slope factor $\mathrm{k}$ and k-estimates of individual IHCs illustrate comparable voltage sensitivity between both conditions ( $p=0.67$, t-test). (D) Average peak-normalized $\mathrm{Ca}^{2+}$-currents resulting from $200 \mathrm{~ms}$ depolarizations to -14 $\mathrm{mV}$. We observe an enhanced inactivation in ribbonless IHCs, quantified as a reduced residual $\mathrm{Ca}^{2+}$-current (inset). ( $n=10$ cells, $\mathrm{N}=5$ for RBE ${ }^{\mathrm{KO} / \mathrm{KO}}$ and $n=11$ cells, $N=7$ in the RBE ${ }^{W T} / \mathrm{WT} ; \mathrm{p}=0.017$, Mann-Whitney-Wilcoxon test).

not shown). The average voltage-sensitivity of activation (slope factor $\mathrm{k}$ ) was not altered $(5 \mathrm{mM}$ $\left[\mathrm{Ca}^{2+}\right]_{e}: \mathrm{p}=0.67$, t-test, Figure $\left.5 \mathrm{C}_{i i}\right)$. Together, this suggests a RIBEYE/ribbons-mediated regulation of IHC Ca ${ }^{2+}$-channels affecting their voltage-range of operation as well as their inactivation kinetics.

We then used the low-affinity $\mathrm{Ca}^{2+}$-indicator dye Fluo-4FF $(800 \mu \mathrm{M})$ to study $\mathrm{Ca}^{2+}$-influx at individual IHC AZs (Frank et al., 2009) using a spinning-disk confocal microscope that allows rapid registering and recording of the majority of the IHC synapses (Figure 6A, Ohn et al., 2016). We chose conditions in which the $\mathrm{Ca}^{2+}$-indicator fluorescence approximates synaptic $\mathrm{Ca}^{2+}$-influx (Frank et al., 


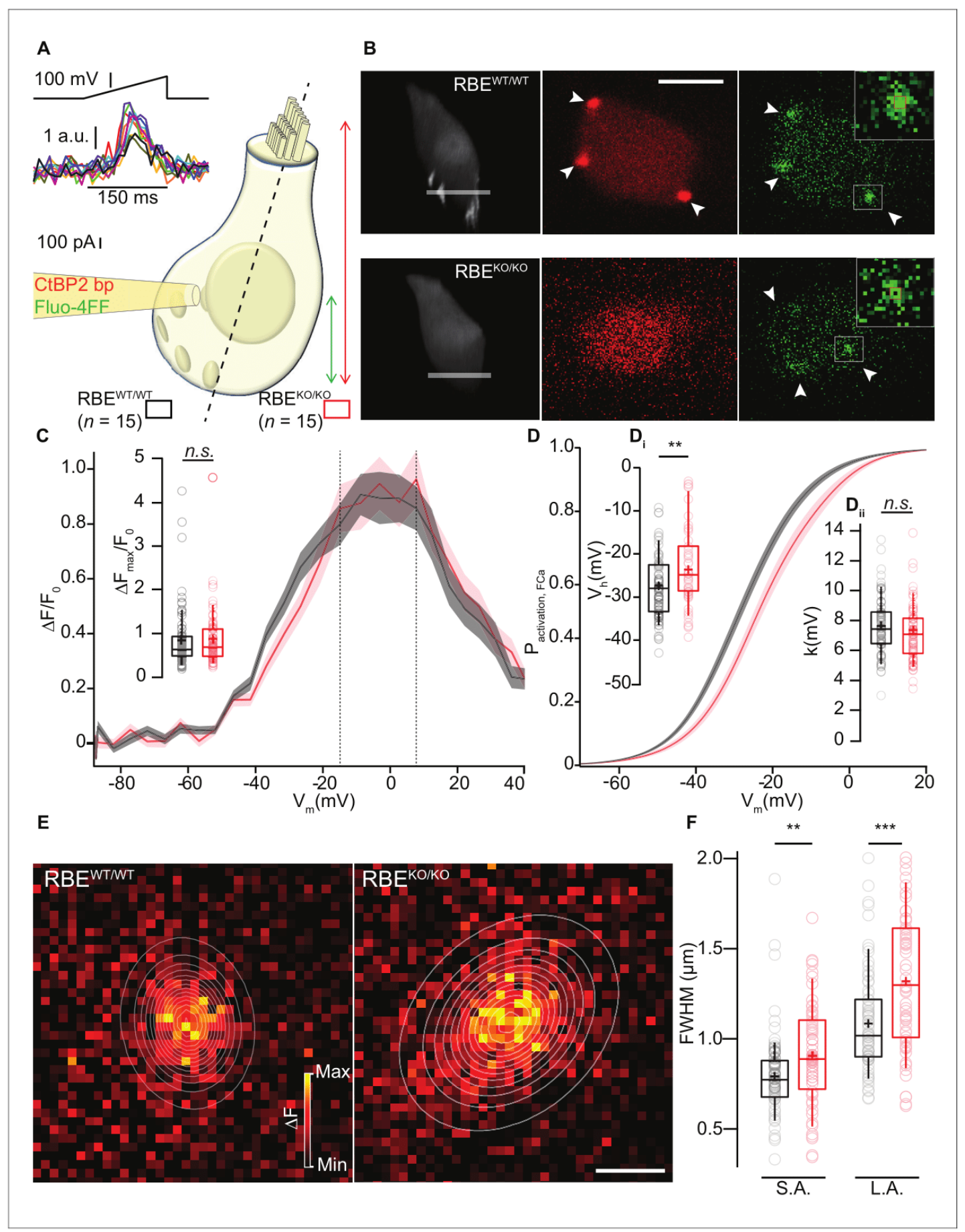

Figure 6. Synaptic $\mathrm{Ca}^{2+}$-influx shows normal amplitude but shifted voltage-dependence and broader spread. (A, B) IHCs were patch-clamped at the modiolar basolateral face, loaded with TAMRA-CtBP2-binding peptide and the low affinity $\mathrm{Ca}^{2+}$-indicator Fluo-4FF, and scanned in the red channel after loading for 4 min to image TAMRA-labeled ribbons, nuclei, and cytosol. 3D projection of TAMRA fluorescence shows the absence of ribbons in $\mathrm{RBE}{ }^{\mathrm{KO} / \mathrm{KO}} \mathrm{IHCs}$ (B: $3 \mathrm{D}$ projection and red channel). Voltage-ramps from -87 to $+63 \mathrm{mV}$ during $150 \mathrm{~ms}$ ( $\mathrm{A}$ : left top) were used to trigger Figure 6 continued on next page 
Figure 6 continued

synaptic hotspots of Fluo-4FF fluorescence (A: left middle, $10 \mathrm{AZs}$ in one exemplary RBEWT/WT IHC, B: green channel, marked by arrowheads; $\Delta \mathrm{F}$ : average of the nine brightest pixels (red square)) and IHC Ca ${ }^{2+}$-influx (A, left bottom). $\mathrm{Ca}^{2+}$-imaging proceeded from the IHC bottom to the most apical

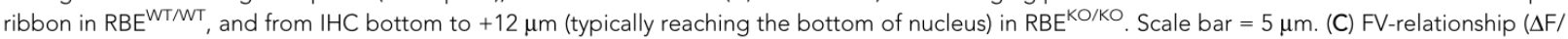
$\mathrm{F}_{0}$ vs. depolarization level in ramp, protocol as in A): approximating the voltage-dependence of synaptic $\mathrm{Ca}^{2+}{ }^{2}$-influx.Mean (line) $\pm \mathrm{S}$.E.M. (shaded areas) are displayed as for (D). (Ci) $\Delta F_{\text {max }} / F_{0}$ was calculated by averaging 5 values at the FV-peak (between the dotted lines) and was comparable between $\operatorname{RBE}^{\text {WT }}$ WT ( $n=78$ AZs for 15 cells, $N=8$ ) and RBE ${ }^{K O} / K_{I H C s}(n=61$ AZs for 15 cells, $N=7)$ ( $p=0.20$, Mann-Whitney-Wilcoxon test). Box plots show 10, $25,50,75$ and $90^{\text {th }}$ percentiles with individual data points overlaid, means are shown as crosses, as for (D, F). (D) Fractional activation curves derived from fits to the FV-relationships (C) were fitted to a Boltzmann function. Mean (line) \pm S.E.M. (shaded areas) are displayed. (Di) The voltage for halfmaximal activation $V_{h}$ was significantly different between $\mathrm{RBE}^{\mathrm{WT} / \mathrm{WT}}(n=68 \mathrm{AZs}$ for $15 \mathrm{IHCs}, \mathrm{N}=8)$ and RBE $\mathrm{KO} / \mathrm{KO}$ ( $n=55 \mathrm{AZs}$ for $15 \mathrm{IHCs}, \mathrm{N}=7$ ) AZs $(p=0.0029$, t-test), while the voltage-sensitivity or slope factor $k$ (Dii) not ( $p=0.42$, t-test). (E) Exemplary $\Delta F$ pictures of Fluo-4FF hotspots at RBEWT/WT (left) and RBE KO/KO (right) synapses fitted and overlaid by 2D-Gaussian functions to estimate spatial extent as full width at half maximum (FWHM) for the short axis (S.A.) and the long axis (L.A.). Scale bar $=1 \mu \mathrm{m}$. (F) Ribbonless synapses of RBE ${ }^{\mathrm{KO} / \mathrm{KO}}$ IHCs showed a greater spatial spread of the Fluo4FF fluorescence change. FWHM calculated from the Gaussian fitting to the Fluo-4FF fluorescence hotspot was larger for both axes in RBE ${ }^{\mathrm{KO} / \mathrm{KO}}(\mathrm{n}=61$ AZs for $15 \mathrm{IHCs}, \mathrm{N}=8$ ) compared to RBE ${ }^{\mathrm{WT} / W T}$ ( $n=74$ AZs for $15 \mathrm{IHCs}, \mathrm{N}=7$ ) (L.A.: $p=0.00016 ; \mathrm{S} . \mathrm{A} .: \mathrm{p}=0.0029$, t-test). DOl: https://doi.org/10.7554/eLife.29275.011

The following figure supplement is available for figure 6:

Figure supplement 1. Semi-quantitative immunofluorescence analysis of the three main proteinaceous $\mathrm{Ca}^{2+}-$ buffers. DOl: https://doi.org/10.7554/eLife.29275.012

2009; Ohn et al., 2016) and henceforth refer to synaptic $\mathrm{Ca}^{2+}$-influx when describing observations based on hotspots of $\mathrm{Ca}^{2+}$-indicator fluorescence at the basolateral IHC membrane. Prior to analysis of synaptic $\mathrm{Ca}^{2+}$-influx, we imaged fluorescently-conjugated CtBP2-binding peptide (Zenisek et al., 2004), which bound to the ribbon-occupied AZs in RBE ${ }^{W T / W T} I H C s$ while it only caused nuclear and diffuse cytosolic fluorescence in the ribbonless $\mathrm{RBE}^{\mathrm{KO} / \mathrm{KO}} \mathrm{IHCs}$ (Figure 6B). We then employed ramp-depolarizations to assess amplitude and voltage-dependence of $\mathrm{Ca}^{2+}$-influx at the synapses located in the subnuclear, basal part of the IHCs (Figure 6A). We found comparable maximal amplitudes of the baseline-normalized fluorescence change $\left(\Delta F / F_{0}, 0.88 \pm 0.08, S . D .=0.66 ; n=61 \mathrm{AZs}\right.$ in $15 \mathrm{IHCs}, \mathrm{N}=7$ for RBE ${ }^{\mathrm{KO} / \mathrm{KO}}$ vs. $0.85 \pm 0.08$, S.D. $=0.68 ; n=78 \mathrm{AZs}$ in $15 \mathrm{IHCs}, \mathrm{N}=8$ for RBE ${ }^{\mathrm{WT} / \mathrm{WT}}$; $\mathrm{p}=0.20$, Mann-Whitney-Wilcoxon test; Figure $6 \mathrm{C}$ ). This is compatible with an unaltered number of synaptic $\mathrm{Ca}^{2+}$-channels at $\mathrm{AZs}$ of $\mathrm{RBE}{ }^{\mathrm{KO} / \mathrm{KO}} \mathrm{IHCs}$ and consistent with our observations of norma whole-cell $\mathrm{Ca}^{2+}$-current amplitudes. As previously reported (Frank et al., 2009; Ohn et al., 2016), there was a substantial variation of the maximal $\Delta F / F_{0}$ among the $A Z s$, which was also comparable between $\mathrm{AZs}$ of both genotypes (c.v. $=0.75$ for $\mathrm{RBE}^{\mathrm{KO} / \mathrm{KO}}$ vs. c.v. $=0.80$ for RBE ${ }^{\mathrm{WT} / \mathrm{WT}}$ ).

$\mathrm{Next}$, we analyzed the voltage-dependence of activation for the synaptic $\mathrm{Ca}^{2+}$-influx as previously described (Ohn et al., 2016). Analysis of fractional activation revealed a depolarized shift in $V_{h}$ by on average $5 \mathrm{mV}$ in RBE ${ }^{\mathrm{KO} / \mathrm{KO}} \mathrm{IHCs}(-22.76 \pm 1.25 \mathrm{mV}, \mathrm{S} . \mathrm{D} .=9.26 \mathrm{mV} ; n=55 \mathrm{AZs}$ in $15 \mathrm{IHCs}, \mathrm{N}=7$ for RBE $\mathrm{KO} / \mathrm{KO}_{\text {vs. }}-27.37 \pm 0.90 \mathrm{mV}$, S.D. $=7.48 \mathrm{mV} ; n=68 \mathrm{AZs}$ in $15 \mathrm{IHCs}, \mathrm{N}=8$ for RBE WT/WT; $p=0.0029$, t-test; Figure $6 D, D i)$, while the slope factor of voltage-dependent activation was unaltered $\left(p=0.42\right.$, t-test, Figure $\left.6 D_{i i}\right)$. Such a shift in the operating range of synaptic $\mathrm{Ca}^{2+}$-influx is expected to alter spontaneous and sound-evoked transmitter release (see below and Ohn et al., 2016). Finally, we studied the spatial extent of the synaptic $\mathrm{Ca}^{2+}$-signals and estimated Full Width Half Maximum (FWHM) by fitting 2D Gaussian functions to the hotspots of $\mathrm{Ca}^{2+}$-indicator fluorescence and found a greater spread of $\mathrm{Ca}^{2+}$-signals at $\mathrm{RBE}^{\mathrm{KO} / \mathrm{KO}} \mathrm{AZs}$ (Figure 6E,F; long axis (L. A.) $=1317 \pm 49 \mathrm{~nm}$, S.D. $=384 \mathrm{~nm}$, short axis (S.A.) $=906 \pm 36 \mathrm{~nm}$, S.D. $=284 \mathrm{~nm} ; n=61$ AZs in 15 IHCs, $N=7$ vs. L.A. $=1083 \pm 33 \mathrm{~nm}$, S.D. $=283 \mathrm{~nm} ;(p=0.00016$, t-test), S.A. $=793 \pm 27 \mathrm{~nm}, \mathrm{~S}$. D. $=233 \mathrm{~nm},\left(p=0.0029\right.$, t-test); $n=74 \mathrm{AZs}$ in $15 \mathrm{IHCs}, \mathrm{N}=8$ for RBE $\left.{ }^{\mathrm{WT} / \mathrm{WT}}\right)$. This larger spread of the presynaptic $\mathrm{Ca}^{2+}$-signals is in agreement with the presence of several Cav1.3-immunofluorescent clusters at $\mathrm{RBE}^{\mathrm{KO} / \mathrm{KO}}$ synapses. In order to exclude lower $\mathrm{IHC} \mathrm{Ca}{ }^{2+}$-buffering to contribute to the observed larger spread of presynaptic $\mathrm{Ca}^{2+}$-signals, we performed semi-quantitative immunofluorescence analysis for the three major cytosolic $\mathrm{Ca}^{2+}$-buffers, the EF-hand $\mathrm{Ca}^{2+}$-binding proteins parvalbumin- $\alpha$, calretinin and calbindin-28k (Pangršič et al., 2015). We did not find any significant differences in their immunofluorescence intensity between IHCs of both genotypes (in arbitrary units, parvalbumin intensity: $2.24 \pm 0.15$, S.D. $=1.04$ for RBE ${ }^{\mathrm{KO} / \mathrm{KO}}$ vs. $1.88 \pm 0.15, \mathrm{~S} . \mathrm{D}=1.01$ for $\mathrm{RBE}^{\mathrm{WT} /}$ WT,$p=0.08$; calbindin intensity: $0.82 \pm 0.06$, S.D. $=0.43$ for RBE ${ }^{\mathrm{KO} / \mathrm{KO}}$ vs. $0.95 \pm 0.07, \mathrm{~S} . \mathrm{D} .=0.49$ for 
$\mathrm{RBE}^{\mathrm{WT} / \mathrm{WT}}, \mathrm{p}=0.23$; calretinin intensity: $0.91 \pm 0.04$, S.D. $=0.26$ for $\mathrm{RBE}^{\mathrm{KO} / \mathrm{KO}}$ vs. $0.82 \pm 0.04$, S. D. $=0.28$ for RBE ${ }^{W T} / W T, p=0.09 ; n=49$ cells and $N=4$ for both conditions, Mann-Whitney-Wilcoxon test for all; Figure 6-figure supplement 1).

\section{IHC exocytosis is normal for strong depolarizations but mildly reduced for weak ones}

The ribbon has been proposed to play a crucial role in the exocytosis of SVs at the IHC AZ (Khimich et al., 2005). Therefore, we monitored stimulated exocytosis of SVs with perforated-patch whole-cell recordings of exocytic membrane capacitance changes $\left(\Delta C_{m}\right)$. Using IHCs from 2/3-weekold $\mathrm{RBE}^{\mathrm{WT} / \mathrm{WT}}$ and $\mathrm{RBE}^{\mathrm{KO} / \mathrm{KO}}$ mice, we found that $\Delta \mathrm{C}_{\mathrm{m}}$ in response to step-depolarizations to the potential that elicits maximal $\mathrm{Ca}^{2+}$-influx $(-14 \mathrm{mV})$ were not different between IHCs with or without ribbons. Both, fast exocytosis elicited by depolarizations of up to $20 \mathrm{~ms}$, attributed to the fusion of the readily releasable pool of SVs (RRP, Moser and Beutner, 2000), and longer stimuli, thought to reflect sustained exocytosis, ongoing $\mathrm{SV}$ replenishment and fusion, were unaltered in $\mathrm{RBE}^{\mathrm{KO} / \mathrm{KO}}{ }_{\mathrm{IHCS}}$ (Figure $7 A, B, C$ ). On average, $\Delta C_{m}$ induced by $20 \mathrm{~ms}$ long maximal $\mathrm{Ca}^{2+}$-influx was $16.70 \pm 1.67 \mathrm{fF}$ (S.D. $=5.80 \mathrm{fF} ; n=12$ cells, $\mathrm{N}=7$ ) for $\mathrm{RBE}^{\mathrm{KO} / \mathrm{KO}}$ compared to $15.22 \pm 0.98 \mathrm{fF}$ (S.D. $=3.26 \mathrm{fF} ; n=11$ cells, $\mathrm{N}=8$ ) for RBE ${ }^{\mathrm{WT} / \mathrm{WT}}$. Exocytic $\Delta \mathrm{C}_{\mathrm{m}}$ elicited by $200 \mathrm{~ms}$ long maximal $\mathrm{Ca}^{2+}$-influx (same $\mathrm{IHCs}$ as for $20 \mathrm{~ms}$ ), on average, amounted to $62.09 \pm 5.40 \mathrm{fF}$ (S.D. $=18.70 \mathrm{fF})$ for $\mathrm{RBE}^{\mathrm{KO} / \mathrm{KO}}$ versus $63.28 \pm 6.64 \mathrm{fF}(\mathrm{S} . \mathrm{D} .=22.04 \mathrm{fF})$ for RBE ${ }^{\mathrm{WT} / \mathrm{WT}}$.

Moreover, trains of 20 step-depolarizations to $-17 \mathrm{mV}$ of $20 \mathrm{~ms}$ pulse duration did not reveal impaired exocytosis in RBE ${ }^{\mathrm{KO} / \mathrm{KO}} \mathrm{HC}$, even when the inter-stimulus interval time was as short as 160 ms (Figure 7D; $n=11$ cells, $\mathrm{N}=5$ for $\mathrm{RBE}^{\mathrm{WT} / \mathrm{WT}}$ and $n=13$ cells, $\mathrm{N}=8$ for $\mathrm{RBE}^{\mathrm{KO} / \mathrm{KO}}$ ). We further explored RRP recovery from partial depletion using a paired-pulse protocol (two strong $20 \mathrm{~ms}$ depolarizations to $-14 \mathrm{mV}$ separated by $50,110,260$ and $510 \mathrm{~ms}$ inter-pulse intervals; Figure 7E,F). RRP recovery, estimated as the $\Delta C_{m}$ ratio of the second and the first pulse, was not altered in $R E^{\mathrm{KO} / K O}$ IHCs at least when probing RRP exocytosis with maximal $\mathrm{Ca}^{2+}$-influx from a hyperpolarized resting potential (Figure 7F). These data are in strong contrast to our previous findings in IHCs of bassoon mutant mice, which we had equivalently analyzed. There, the loss of synaptic ribbons, combined with a loss of functional bassoon resulted in profound deficits in exocytosis (Khimich et al., 2005 Frank et al., 2010; Jing et al., 2013).

Given the finding of a small depolarized shift in the operating range of $\mathrm{Ca}^{2+}$-channels in $\mathrm{RBE}^{\mathrm{KO} /}$ KO IHCs (Figure 6D), we also probed the voltage-dependence of $\Delta C_{m}$ elicited by $100 \mathrm{~ms}$ step-depolarizations (Figure $7 G, H$ ). In agreement with the results obtained at maximal $\mathrm{Ca}^{2+}$-influx, we did not find significant differences in $\Delta \mathrm{C}_{\mathrm{m}}$ for stronger depolarizations (e.g. pulses to $-39 \mathrm{mV}$ elicited an average $\Delta C_{m}$ of $20.67 \pm 7.46 \mathrm{fF}, \mathrm{S} . \mathrm{D} .=23.58 \mathrm{fF}, n_{\min }=10 \mathrm{lHCs}, \mathrm{N}=9$ for $\mathrm{RBE}^{\mathrm{KO} / \mathrm{KO}} \mathrm{vs} .24 .12 \pm 4.04$ $\mathrm{fF}, \mathrm{S} . \mathrm{D} .=13.98 \mathrm{fF}, \mathrm{n}_{\min }=10 \mathrm{HCS}, \mathrm{N}=9$ for RBEWT/WT; $p=0.20$; Mann-Whitney-Wilcoxon test). However, for weaker depolarizations in the range of physiological receptor potentials (Russell and Sellick, 1983), we observed a subtle but significant reduction in exocytosis for RBE ${ }^{\mathrm{KO} / K O} \mathrm{IHCs}$ (Figure $7 \mathrm{H}, \mathrm{p}=0.0115, \mathrm{p}=0.0295$ and $\mathrm{p}=0.1321$ for $-45,-43$ and $-41 \mathrm{mV}$; without definitive outliers as determined by Graphpad Prism: $p=0.0017, p=0.0042$ and $p=0.0489$, respectively; Mann-WhitneyWilcoxon test for all). For instance, depolarization to $-45 \mathrm{mV}$ elicited a $\Delta \mathrm{C}_{\mathrm{m}}$ of $4.79 \pm 2.26 \mathrm{fF}$ for $\mathrm{RBE}^{\mathrm{KO} / \mathrm{KO}}$ (S.D. $=7.14 \mathrm{fF} ; \mathrm{n}_{\min }=10$ cells, $\mathrm{N}=9$ ) compared to $9.85 \pm 1.60 \mathrm{fF}$ for RBE ${ }^{\mathrm{WT} / \mathrm{WT}}$ (S. D. $=5.05 \mathrm{fF} ; n_{\min }=10$ cells, $\left.\mathrm{N}=8\right)$. The $\mathrm{Ca}^{2+}$-current integral $\left(\mathrm{Ca}^{2+}\right.$-charge, $\left.\mathrm{Q}_{\mathrm{Ca}}\right)$, as well, tended to be reduced for $\mathrm{RBE}^{\mathrm{KO} / \mathrm{KO}} \mathrm{IHC}$ at these mild depolarizations, which, however, did not reach statistical significance (e.g. $\mathrm{Q}_{\mathrm{Ca}}$ for $-45 \mathrm{mV}: 3.90 \pm 0.49 \mathrm{pC}$, S.D. $=1.54 \mathrm{pC}$ for $\mathrm{RBE}^{\mathrm{KO} / \mathrm{KO}}$ vs. $5.15 \pm 0.54 \mathrm{pC}$, S.D. $=1.72 \mathrm{pC}$ for RBE ${ }^{\mathrm{WT} / \mathrm{WT}} ; \mathrm{p}=0.1053$; t-test). In summary, we found exocytosis to be unaltered for strong depolarizations but mildly decreased for more physiological stimuli in $\mathrm{RBE}^{\mathrm{KO} / \mathrm{KO}} \mathrm{IHCs}$, which is in line with the findings of the companion paper by Becker et al..

\section{Lack of synaptic ribbons impairs synchronous activation of the auditory pathway}

Next, we studied sound encoding in $\mathrm{RBE}^{\mathrm{KO} / \mathrm{KO}}$ mice in vivo. First, we recorded auditory brainstem responses (ABR) and found a significant reduction in the amplitude of wave I that reflects the SGN compound action potential $\left(1.14 \pm 0.13 \mu \mathrm{V}, \mathrm{S} . \mathrm{D} .=0.38 \mu \mathrm{V}, \mathrm{N}=10\right.$ for $\mathrm{RBE}^{\mathrm{KO} / \mathrm{KO}} \mathrm{vs} .3 .30 \pm 0.51 \mu \mathrm{V}$, S.D. $=1.54 \mu \mathrm{V}, \mathrm{N}=10$ for RBE ${ }^{\mathrm{WT}} / \mathrm{WT}, \mathrm{p}=0.0007, \mathrm{NPMC}$ test). This indicates less synchronous $\mathrm{SGN}$ 


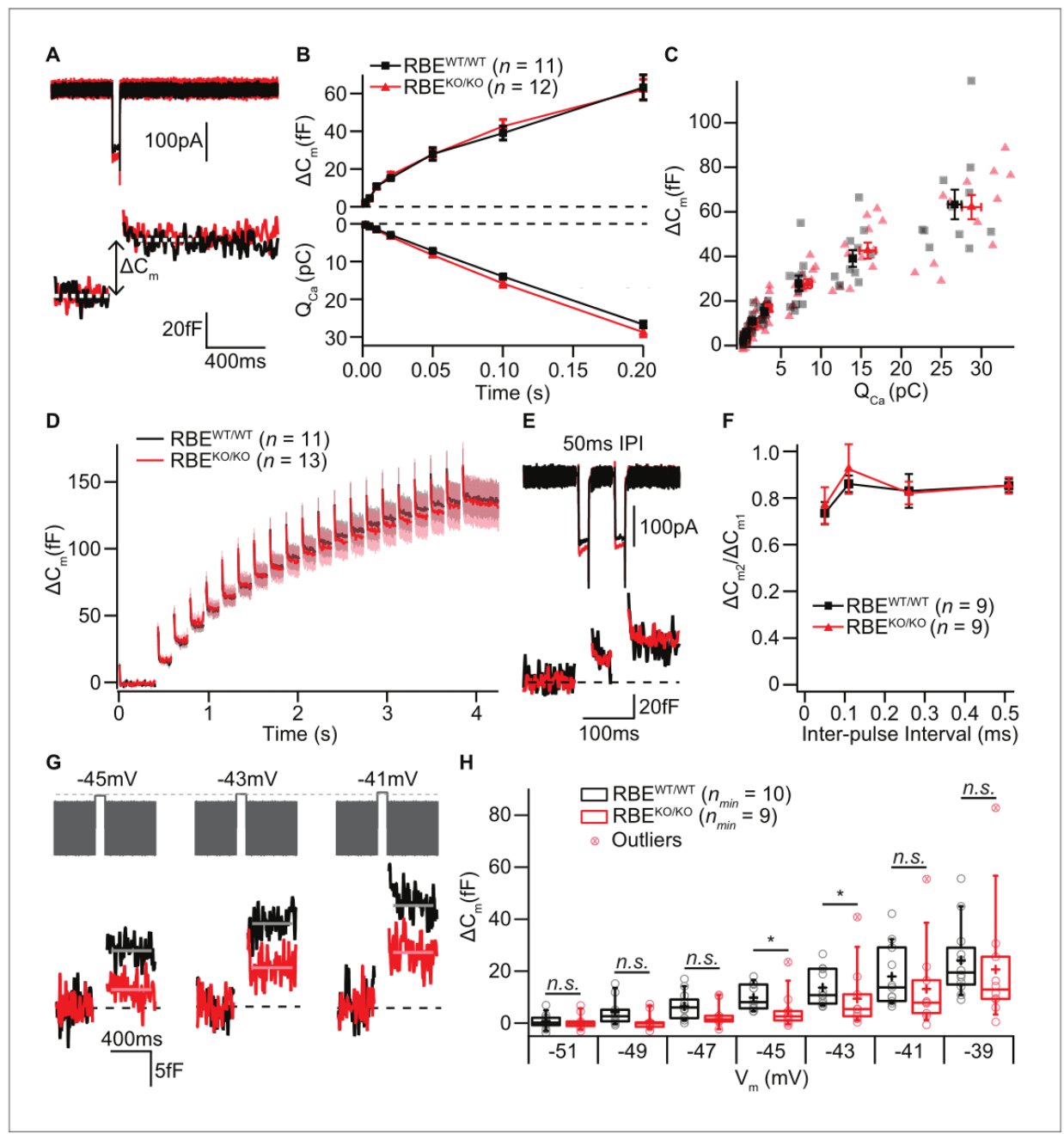

Figure 7. $\mathrm{IHC}$ exocytosis is normal for strong depolarizations but mildly reduced for weak ones. (A) Representative $\mathrm{Ca}^{2+}$-currents (top) and corresponding low passed-filtered membrane capacitance $\left(\Delta \mathrm{C}_{m}\right)$ traces recorded from RBE ${ }^{W T} / \mathrm{WT}$ and RBE ${ }^{\mathrm{KO} / K O}$ IHCs upon $50 \mathrm{~ms}$ depolarizations from -69 to $-14 \mathrm{mV}$. (B) Cumulative exocytosis (exocytic $\Delta \mathrm{C}_{\mathrm{m}}$, top) and corresponding $\mathrm{Ca}^{2+}$-charge $\left(\mathrm{Q}_{\mathrm{Ca}}\right.$, bottom) of RBEWT/WT ( $n=11 \mathrm{cells}$, $\mathrm{N}=8$ ) and $\mathrm{RBE}^{\mathrm{KO} / \mathrm{KO}}$ ( $n=12$ cells, $\mathrm{N}=7$ ) IHCs as a function of stimulus duration ( 2 to $200 \mathrm{~ms}$ to $-14 \mathrm{mV}$ ) were unaltered in RBE ${ }^{\mathrm{KO} / \mathrm{KO}} \mathrm{IHCs}$. Data is presented as mean \pm S.E.M as for $(F)$. (C) Relating $\Delta C_{m}$ to the corresponding $Q_{C a}$ indicated comparable $\mathrm{Ca}^{2+}$ efficiency of exocytosis between RBE ${ }^{\text {WT }}$ WT and RBE ${ }^{\mathrm{KO} /}$ $\mathrm{KO}$ IHCs. Mean \pm S.E.M. for each pulse duration is presented in black and red; individual IHCs data points are overlaid. (D) Mean $\Delta \mathrm{C}_{\mathrm{m}}$ traces (shaded areas: S.E.M.) in response to trains of $20 \mathrm{~ms}$ depolarizations from -87 to $-17 \mathrm{mV}$ (20 stimuli separated by $160 \mathrm{~ms}$ ) of RBE ${ }^{\mathrm{WT}} / \mathrm{WT}$ ( $n=11$ cells, $\mathrm{N}=5$ ) and $\mathrm{RBE}^{\mathrm{KO} / \mathrm{KO}}\left(n=13\right.$ cells, $\mathrm{N}=8$ ) IHCs show comparable exocytic $\Delta \mathrm{C}_{\mathrm{m}}$. (E) Representative low pass-filtered $\Delta \mathrm{C}_{\mathrm{m}}$ traces in response to a pair of $20 \mathrm{~ms}$ pulses to $-17 \mathrm{mV}$, separated by a $50 \mathrm{~ms}$ of inter-pulse interval (IPI). (F) Ratios of exocytosis $\left(\Delta \mathrm{C}_{\mathrm{m} 2} / \Delta \mathrm{C}_{\mathrm{m} 1}\right)$ to a pair of $20 \mathrm{~ms}$ pulses with varying interpulse intervals $\left(50,110,260\right.$ and $510 \mathrm{~ms}$ ) reveal a comparable recovery from RRP depletion between RBE ${ }^{\mathrm{WT} / \mathrm{WT}}(n=9 \mathrm{cells}, \mathrm{N}=6)$ and RBE ${ }^{\mathrm{KO} / \mathrm{KO}}$ IHCs $\left(n=9\right.$ cells, $N=6$ ). (G) Representative low pass-filtered $\Delta C_{m}$ traces in response to $100 \mathrm{~ms}$ step-depolarizations to $-45,-43$ and $-41 \mathrm{mV}$. (H) Box plot and single values of $\Delta \mathrm{C}_{m}$ elicited by 100 ms step-depolarizations of RBE ${ }^{\mathrm{WT} / W T}$ ( $n_{\min }=10$ cells, $\mathrm{N}=8$ ) and RBE ${ }^{\mathrm{KO} / \mathrm{KO}}$ ( $n_{\min }=9 \mathrm{cells}, \mathrm{N}=9$ ) IHCs to different potentials. Exocytic $\Delta C_{m}$ of $R B E^{K O} / K O I H C s$ was reduced for mild depolarizations $(-45,-43$ and $-41 \mathrm{mV} ; p=0.0115, p=0.0295$ and $p=0.1321$, respectively; $p=0.0017, p=0.0042$ and $p=0.0489$, without definitive outliers; Mann-Whitney-Wilcoxon test), but comparable to RBE ${ }^{W T} / \mathrm{WT}$ IHCs at stronger depolarizations ( $-39 \mathrm{mV} ; \mathrm{p}=0.2030$, Mann-Whitney-Wilcoxon test). Box plots show $10,25,50,75$ and $90^{\text {th }}$ percentiles with the individual data points overlaid.

DOI: https://doi.org/10.7554/eLife.29275.013 
activation in the absence of synaptic ribbons (Figure $8 A, B$ ). The subsequent $A B R$ waves (Figure 8figure supplement 1) were normal in amplitude (waves II, IV and V, while wave III was reduced) indicating a degree of central auditory compensation for the sound encoding deficit, for example via coincidence detection of converging SGN input in the cochlear nucleus (Joris et al., 1994;

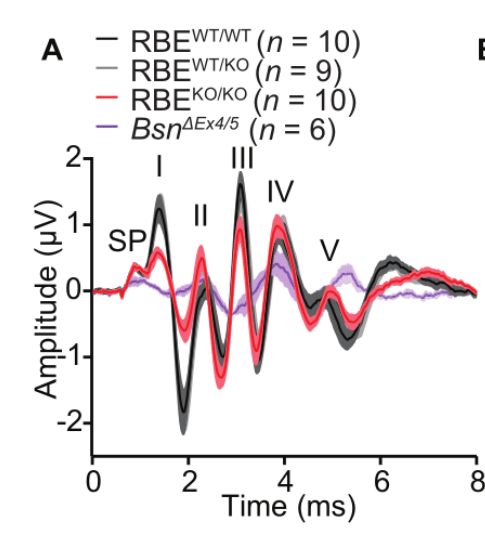

C
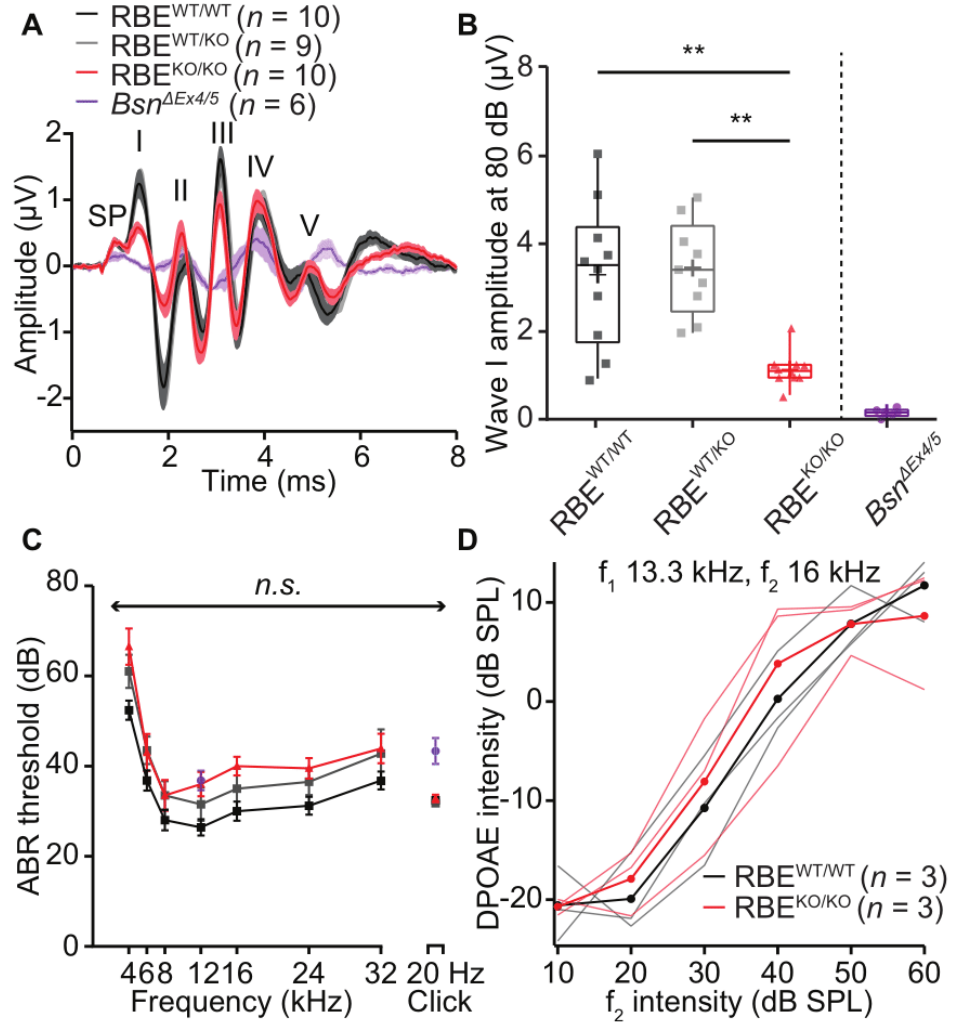

Figure 8. Auditory brainstem responses indicate impaired synchronous $S G N$ activation in $R B E^{\mathrm{KO} / K O}$ mice. (A) Average $A B R$ waveforms in response to $80 \mathrm{~dB}$ clicks $\left(n=\mathrm{N}=10\right.$ for $\mathrm{RBE}^{\mathrm{KO} / K O}$ and RBEWT/WT, $N=9$ for RBE ${ }^{\mathrm{WT} / K O}$ at 6 weeks of age) showed a reduced amplitude of $A B R$ wave I in $\mathrm{RBE}^{\mathrm{KO} / \mathrm{KO}}$ mice indicating an impairment of SGN activation, which is quantified in (B). This decrease was not as pronounced as in SGNs lacking bassoon (Bsn ${ }^{\Delta E \times 4 / 5}$, data from Jing et al., 2013). The central ABR waves were better preserved, except for wave III. SP: summating potential (hair cell receptor potential), roman numerals (I-V): ABR waves generated along the early auditory pathway. Mean (lines) \pm S.E.M. (shaded areas) are displayed. (B) ABR wave I was significantly reduced in RBE ${ }^{\mathrm{KO} / \mathrm{KO}}$ mice as compared to $\operatorname{RBE}^{\mathrm{WT} / W T}$ and $\operatorname{RBE}^{\mathrm{WT} / K O}$ ( $p=0.0051$ and $p=0.0017$, respectively, NPMC test). No statistical significance was observed between responses recorded in $\operatorname{RBE}^{\mathrm{WT} / W T}$ and $\mathrm{RBE}^{\mathrm{WT} / K O}$ mice $(\mathrm{p}>0.9999, \mathrm{NPMC}$ test). Data from $B s n^{\Delta E \times 4 / 5}$ are shown for comparison. Box plots show 10, 25, 50, 75 and $90^{\text {th }}$ percentiles with the individual data points overlaid, means are shown as crosses. (C) ABR thresholds were comparable in RBE ${ }^{\text {WT/WT }}$ $\mathrm{RBE}^{\mathrm{WT} / \mathrm{KO}}$ and RBE ${ }^{\mathrm{KO} / K O}$ for tone burst-driven (n.s., Tukey's test) and click-driven ABRs (n.s., NPMC test). Previously published data for $B s n^{\Delta E \times 4 / 5}$ showed elevated thresholds as a response to short stimuli (click $20 \mathrm{~Hz}$ ) but a similar threshold level at $12 \mathrm{kHz}$. (D) DPOAE amplitude in response to pairs of simultaneous sine waves $\left(\mathrm{f}_{1}\right.$ and $\mathrm{f}_{2}$, frequencies indicated on panel) at increasing stimulus intensity ( $f_{1}$ intensity $10 \mathrm{~dB}$ above $f_{2}$ in all cases). Mean (thick lines) and data from individual mice $\left(n=\mathrm{N}=3\right.$ in $\mathrm{RBE}^{\mathrm{WT} / \mathrm{WT}}$ and $\left.\mathrm{RBE}^{\mathrm{KO} / \mathrm{KO}}\right)$ are displayed. DOI: https://doi.org/10.7554/eLife.29275.014

The following figure supplement is available for figure 8:

Figure supplement 1. Quantification of $A B R$ waves $I I-V$ amplitude in $R B E^{K O / K O}$ mice. DOI: https://doi.org/10.7554/eLife.29275.015 
Strenzke et al., 2009). We found a non-significant trend of ABR threshold to be increased across all frequencies in $\mathrm{RBE}^{\mathrm{KO} / \mathrm{KO}}$ mice (approximately $10 \mathrm{~dB}$ across all frequencies, Figure $8 \mathrm{C}$; refer to the companion paper Becker et al. showing significantly increased ABR-thresholds based on a larger sample, $\mathrm{N}=28 \mathrm{RBE}^{\mathrm{KO} / \mathrm{KO}}$ mice vs. $22 \mathrm{RBE}{ }^{\mathrm{WT} / \mathrm{WT}}$ mice). Cochlear amplification, probed by recordings of distortion product otoacoustic emissions (DPOAE, Figure $8 D$ ), was intact in $\mathrm{RBE}{ }^{\mathrm{KO} / \mathrm{KO}}$ mice. Additionally, RBE ${ }^{\mathrm{WT} / K O}$ mice showed no significant changes in $A B R$ wave I amplitudes and $A B R$ thresholds (Figure 8), suggesting that the subtle morphological differences observed for afferent synapses of $\mathrm{RBE}^{\mathrm{WT} / \mathrm{KO}} \mid \mathrm{HC}$ s by electron and confocal-immunofluorescence microscopy did not turn into a deficit of sound coding measurable by ABR recordings. The wave I amplitude reduction and ABR threshold elevation were much less pronounced than in bassoon mutant mice (Khimich et al., 2005; Buran et al., 2010; Jing et al., 2013).

We then turned to in vivo extracellular recordings from single auditory neurons by targeting glass microelectrodes to where the auditory nerve enters the anteroventral cochlear nucleus (AVCN) in the brainstem (Taberner and Liberman, 2005; Jing et al., 2013). 'Putative' SGNs (hereafter dubbed SGN for simplicity) were identified based on the depth of electrode position and their firing response to pure-tone stimulation (primary-like peristimulus time histogram and latency, Figure 9) and analyzed in separation from 'putative' cochlear nucleus neurons (Figure 10). Since all firing of the individual SGN is thought to be driven by transmitter release from a single IHC AZ (Heil et al., 2007; Liberman, 1978; Robertson and Paki, 2002), these recordings provide insight into single AZ function. We first assessed the spontaneous firing activity and found an increased abundance of SGNs with low spontaneous firing rates in $\mathrm{RBE}^{\mathrm{KO} / \mathrm{KO}}$ mice $(72 \%$ with rates $<10 \mathrm{~Hz}, n=43 \mathrm{SGNs}$, $N=9$ vs. $50 \%$ in RBE ${ }^{W T} / W T, n=40$ SGNs, $N=8 ; p=0.0267$, Kolmogorov-Smirnov test; Figure 9A). Frequency tuning was intact in $\mathrm{RBE}^{\mathrm{KO} / \mathrm{KO}} \mathrm{SGNs}$ (Figure $9 \mathrm{~B}$ ): the sharpness of tuning expressed by the $Q_{10 d B}$ (width of tuning curve $10 \mathrm{~dB}$ above threshold at the characteristic frequency $\left(C_{f}\right)$ normalized by $\mathrm{C}_{\mathrm{f}}$ ) was comparable (mean: $9.28 \pm 1.01$, S.D. $=6.32$ and median: 7.41 for RBE ${ }^{\mathrm{KO} / \mathrm{KO}}$ SGNs, $n=39$ SGNs, $N=9$ vs. mean: $12.50 \pm 1.98$, S.D. $=11.91$ and median: 8.36 for RBE ${ }^{W T} / \mathrm{WT}$ SGNs, $n=36$ SGNs, $N=9 ; p=0.28$, Mann-Whitney-Wilcoxon test). However, the sound threshold at $C_{f}$ was significantly elevated by almost $20 \mathrm{~dB}$ in $\mathrm{RBE}{ }^{\mathrm{KO} / \mathrm{KO}}$ mice $(35.60 \pm 3.45 \mathrm{~dB}$ SPL, S.D. $=22.66 \mathrm{~dB} \mathrm{SPL}$ for RBE ${ }^{\mathrm{KO} / \mathrm{KO}} \mathrm{SGNs}, n=43 \mathrm{SGNs}, \mathrm{N}=9$ vs. $16.05 \pm 2.47 \mathrm{~dB}$ SPL, S.D. $=15.42 \mathrm{~dB}$ SPL for RBE ${ }^{\mathrm{WT} / \mathrm{WT}}$ SGNs, $n=39$ SGNs, $N=9, p<0.0001$, Mann-Whitney-Wilcoxon test; Figure 9C). Given the normal frequency tuning and DPOAE, this threshold increase seems unlikely to result from a putative functional cochlear deficit upstream of the IHCs.

Next, we studied the firing response of SGNs to 50 ms tone bursts (at $C_{f}$ and $30 \mathrm{~dB}$ above sound threshold, $200 \mathrm{~ms}$ inter-stimulus interval), which is governed by the presynaptic glutamate release and postsynaptic spike generation. The peak firing rate at sound onset is thought to reflect the initial rate of release from the SV-occupied release sites of the RRP ('standing RRP', [Oesch and Diamond, 2011; Pangršič et al., 2012]). Refractoriness and the decline of release rate due to partial depletion of the standing RRP likely dominate the subsequent spike rate adaptation. Finally, the adapted firing rate reports SV replenishment and subsequent fusion (reviewed in Pangršič et al., 2012; Rutherford and Moser, 2016). We observed reduced spike rates of SGNs from RBE ${ }^{\mathrm{KO} / \mathrm{KO}}$ mice (Figure 9D,E) both at sound onset ( $p=0.0001, n=39 \mathrm{SGNs}, \mathrm{N}=8$ in $\mathrm{RBE}^{\mathrm{KO} / \mathrm{KO}}$ and $n=38 \mathrm{SGNs}$, $\mathrm{N}=9$ in RBE ${ }^{W T} / \mathrm{WT}$, t-test) and after short-term adaptation ( $p=0.0023$, Mann-Whitney-Wilcoxon test). Both, peak and adapted rates were similarly affected by the RIBEYE-disruption, indicated by the scatter plot of peak vs. adapted rates (Figure 9E). A significant peak rate reduction was also observed at higher stimulation frequencies $(10 \mathrm{~Hz}$, Figure $9 \mathrm{G}-H)$. The spike rates were better preserved in RBE ${ }^{\mathrm{KO} / \mathrm{KO}}$ SGNs than in SGNs of bassoon mutant mice ( $B s n^{\Delta e \times 4 / 5}$ data of Jing et al. (2013), purple data in Figure 9G-I). We approximated adaptation within the $50 \mathrm{~ms}$ response by singleexponential fitting since double exponential fitting did not regularly report two temporally discernible components in RBE ${ }^{\mathrm{KO} / \mathrm{KO}} \mathrm{SGNs}$. The mean apparent adaptation time constant reported by single-exponential fitting were significantly slowed in RBE ${ }^{\mathrm{KO} / \mathrm{KO}} \mathrm{SGNs}(9.83 \pm 0.50 \mathrm{~ms}$, S.D. $=2.85 \mathrm{~ms}$, median: $10.46 \mathrm{~ms}, \mathrm{n}=32 \mathrm{SGNs}, \mathrm{N}=8)$ as compared to $\mathrm{RBE}^{\mathrm{WT} / \mathrm{WT}} \mathrm{SGNs}(8.71 \pm 0.50 \mathrm{~ms}$, $\mathrm{S}$. D. $=3.05 \mathrm{~ms}$, median: $8.73 \mathrm{~ms}, n=37 \mathrm{SGNs}, \mathrm{N}=9, p=0.033$, Mann-Whitney-Wilcoxon test). The results of double-exponential fitting of RBE ${ }^{W T} / W T$ and $\mathrm{RBE}^{\mathrm{KO} / K O}$ SGNs support the slowed adaptation kinetics and are presented in Table 1. As expected for the reduced peak firing rate, we found prolonged first spike latency which also showed greater temporal jitter (Figure 9F). The reduced peak firing rate together with increased first spike latency jitter likely explain the reduction in $A B R$ 
A

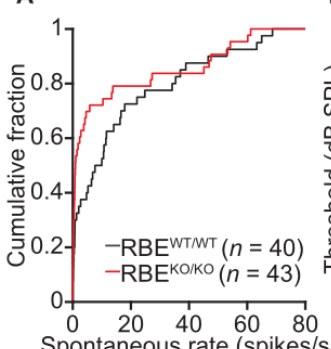

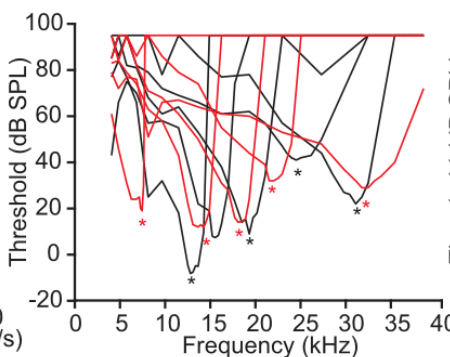

C

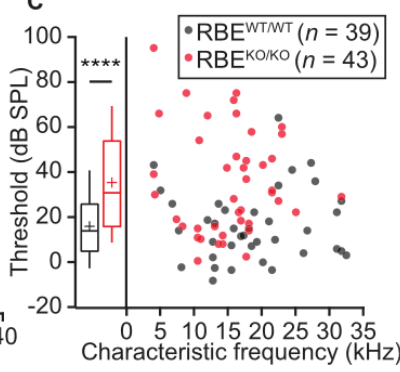

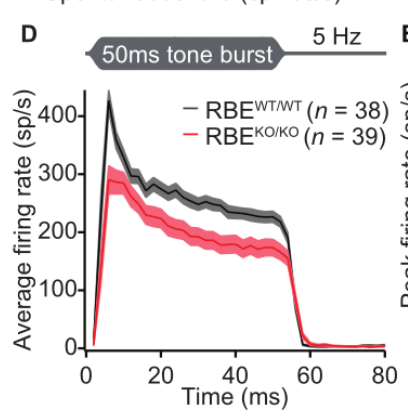

G

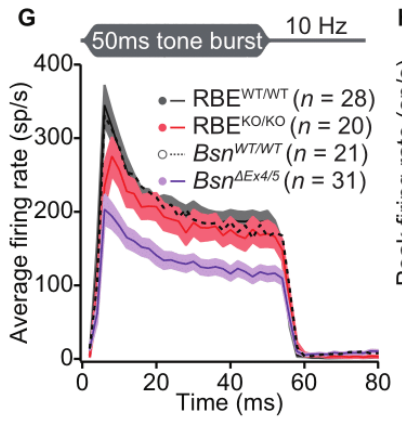

E

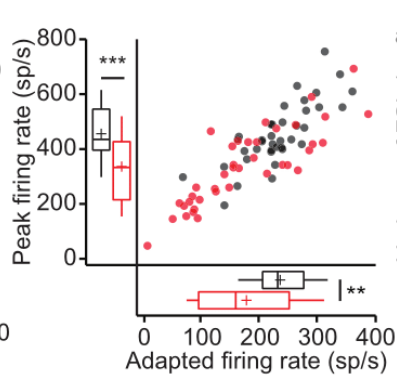

H

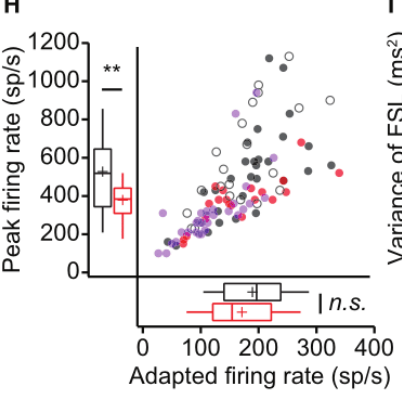

F

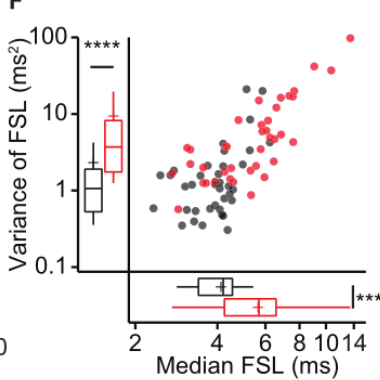

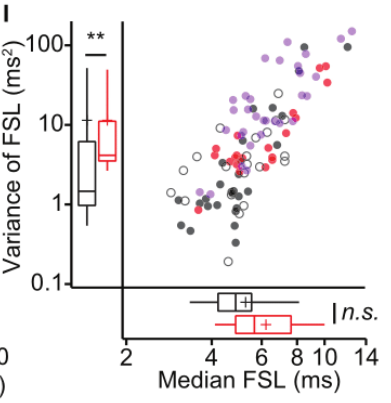

Figure 9. Impaired spontaneous and sound-evoked firing in putative SGNs of RBE ${ }^{\mathrm{KO} / K O}$ mice. (A) Cumulative distribution functions of spontaneous firing rates of putative SGNs showed a higher fraction of low spontaneous rate SGNs in RBE ${ }^{\mathrm{KO} / K O}$ mice $(n=43 \mathrm{SGNs})$ than in RBEWT/WT $\mathrm{mice}(n=40$ SGNs, $N=8$ ) ( $p=0.027$, Kolmogorov-Smirnov test). (B) Representative frequency tuning curves of RBE ${ }^{\mathrm{KO} / \mathrm{KO}}$ and RBE ${ }^{\mathrm{WT}}$ WT $\mathrm{SGNs}$ showed comparable sharpness of tuning between SGNs of both genotypes. * point to the characteristic frequency $\left(\mathrm{C}_{f}\right)$ for which an increase in $\mathrm{SGN}$ firing requires the least sound pressure level. (C) Thresholds at $\mathrm{C}_{f}$ of RBE ${ }^{\mathrm{KO} / \mathrm{KO}} \mathrm{SGNs}\left(n=43 \mathrm{SGNs}, \mathrm{N}=9\right.$ ) were higher than those in RBE ${ }^{W T N T}$ mice $(n=39 \mathrm{SGNs}, \mathrm{N}=9$ ) ( $p<0.0001$, Mann-Whitney-Wilcoxon test). Box plots show 10, 25, 50, 75 and $90^{\text {th }}$ percentiles, means are shown as crosses, as for (E), (F), (H) and (I). (D) Average PSTH (bin width $=2 \mathrm{~ms}$ ) of RBE ${ }^{\mathrm{KO} / K O}(n=39 \mathrm{SGNs}, \mathrm{N}=8)$ and RBEWT/WT SGNs $(n=38 \mathrm{SGNs}, \mathrm{N}=9)$ recorded in response to 50 ms tone bursts at $\mathrm{C}_{\mathrm{f}} 30 \mathrm{~dB}$ above threshold at a stimulus rate of $5 \mathrm{~Hz}$. The time course of adaptation of RBE ${ }^{\mathrm{KO} / \mathrm{KO}} \mathrm{SGNs}$ (calculated by fitting a single exponential function to the individual histograms) was significantly longer ( $p=0.033$, Mann-Whitney-Wilcoxon test). Mean (line) \pm S.E.M. (shaded areas) are displayed as for (G). (E) Scatterplot of peak firing rate (bin with highest rate at sound onset) and adapted firing rate (averaged 35-45 ms from response onset) revealed lower firing rates in $\operatorname{RBE}^{\mathrm{KO} / \mathrm{KO}}(n=39 \mathrm{SGNs})$ as compared to $\mathrm{RBE}^{\mathrm{WT} / \mathrm{WT}}$ ( $\left.n=38 \mathrm{SGNs}, \mathrm{N}=9\right)$ mice (data from (D), peak rate: $p=0.0001$, adapted rate: $p=0.0023$, Mann-Whitney-Wilcoxon test). (F) Increased latency (data from (D), $p=0.0002)$ and variance of latency ( $p<0.0001$, Mann-Whitney-Wilcoxon test) of the first spike after sound onset in RBE ${ }^{K O} / K O$ SGNs indicated lower temporal precision of sound onset coding. (G) Average PSTH (bin width $=2 \mathrm{~ms}$ ) of RBE ${ }^{\mathrm{KO} / \mathrm{KO}}(n=20 \mathrm{SGNs}, \mathrm{N}=6)$ and RBEWT/WT SGNs $(n=28 \mathrm{SGNs}, \mathrm{N}=8)$ were recorded in response to $50 \mathrm{~ms}$ tone bursts at $\mathrm{C}_{\mathrm{f}} 30 \mathrm{~dB}$ above threshold at a stimulus rate of $10 \mathrm{~Hz}$ and showed a similar adapted response in $\mathrm{RBE}^{\mathrm{KO} / \mathrm{KO}}$ as compared to lower stimulation rates (Figures 9,11) but still a lower onset response than in RBEWT/WT SGNs. Responses in Bsn ${ }^{\Delta E \times 4 / 5}$ mutants (shown for comparison, replotted from Jing et al., 2013) were considerably lower. (H) Scatterplot of peak firing rate (bin with highest rate at sound onset) and adapted firing rate (averaged 35-45 ms from response onset) show decreased onset firing rates in $\mathrm{RBE}^{\mathrm{KO} / \mathrm{KO}}$ as compared to RBE ${ }^{\mathrm{WT} / \mathrm{WT}} \mathrm{mice}$ (data from (G), $\mathrm{p}=0.0093$, Mann-Whitney-Wilcoxon test). The adapted response was comparable in both cases ( $p=0.3584$, t-test). Data points from $B s n^{\Delta E \times 4 / 5}$ mutants (re-plotted from Jing et al., 2013) and WT littermates are shown for comparison, also in (I). (I) Increased variance of first spike latency after sound onset in RBE ${ }^{\mathrm{KO} /}$ Figure 9 continued on next page 
Figure 9 continued

KO SGNs ( $p=0.0089$, Mann-Whitney-Wilcoxon test) and comparable latencies were observed at this stimulation frequency (data from ( $G$ ), $p=0.0761$, Mann-Whitney-Wilcoxon test).

DOI: https://doi.org/10.7554/eLife.29275.016

wave I amplitude. The firing of putative AVCN neurons was better preserved: putative bushy cells showed normal sound driven rates and chopper cells only a mild reduction in peak rate (Figure 10).

Next, we explored the encoding of sound intensity by estimating the mean firing rate during 50 ms tone bursts at different sound pressure levels. These 'rate-level functions' (Figure 11A) indicated that the spike rate increase with the sound pressure level $\left(p=0.068, n=24 \mathrm{SGNs}, \mathrm{N}=8\right.$ in $\mathrm{RBE}^{\mathrm{KO} / \mathrm{KO}}$ and $n=19$ SGNs, N = 7 in RBE ${ }^{W T / W T}$, Mann-Whitney-Wilcoxon test, Figure 11-figure supplement $1 \mathrm{~A}$ ) and the dynamic range of sound coding (sound pressure level for which the spike rate changes from 10-90\%, Figure 11-figure supplement 1B, $p=0.3044$, t-test) were not significantly altered. We then used transposed tones $\left(C_{f}\right.$ at $500 \mathrm{~Hz}$ modulation frequency) in order to probe for the temporal fidelity and reliability of firing in $\mathrm{RBE}^{\mathrm{KO} / \mathrm{KO}} \mathrm{SGNs}$ in the steady state (Figure 11D). These experiments corroborated the reduced maximal firing rate of $\mathrm{RBE}^{\mathrm{KO} / \mathrm{KO}} \mathrm{SGNs}(n=22 \mathrm{SGNs}, \mathrm{N}=7$ in $\mathrm{RBE}^{\mathrm{KO} / \mathrm{KO}}$ and $n=15 \mathrm{SGNs}, \mathrm{N}=6$ in $\mathrm{RBE}^{\mathrm{WT} / \mathrm{WT}}, \mathrm{p}<0.0001$, t-test) and indicated that the temporal precision of sound coding is impaired also in the steady state (reduced Synchronization Index: $\mathrm{p}=0.0043$, t-test)

In order to further scrutinize the potential role of the synaptic ribbon in vesicle replenishment, we studied the response to prolonged tone-stimulation (Figure $11 \mathrm{~B}, 500 \mathrm{~ms}$ at $\mathrm{C}_{\mathrm{f}}$ and $30 \mathrm{~dB}$ above threshold, 2 s inter-stimulus interval). The peak rate was better preserved in $\mathrm{RBE}^{\mathrm{KO} / \mathrm{KO}} \mathrm{SGNs}$ than

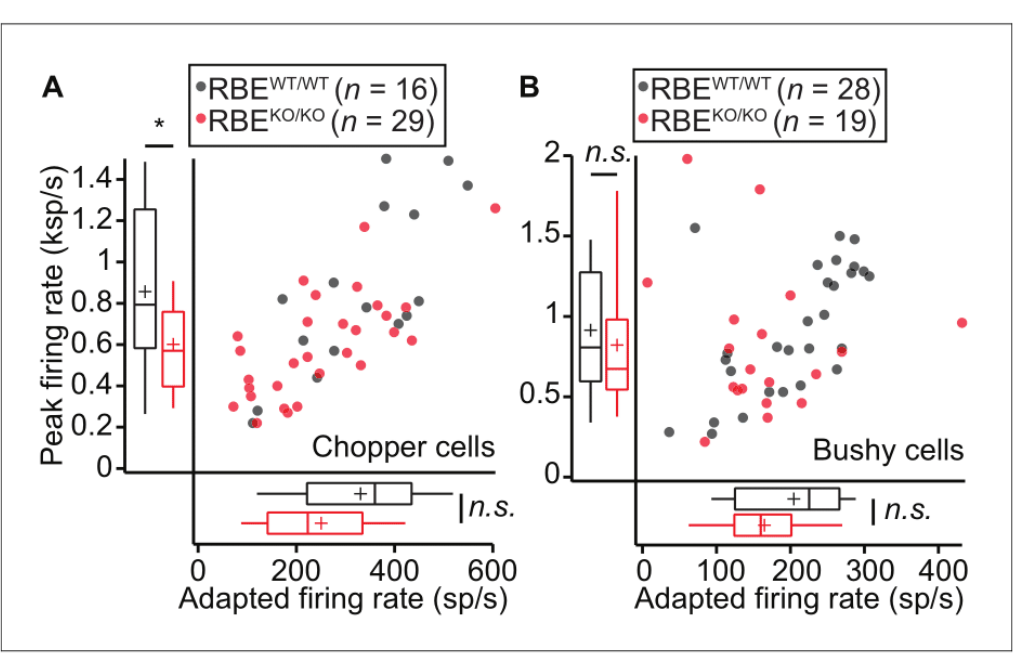

Figure 10. Responses to pure tones in cochlear nucleus cells showed that the mutant phenotype is partially compensated in higher stations of the auditory pathway. (A) Quantification of peak and adapted responses to 50 ms tone burst stimulation, $30 \mathrm{~dB}$ above threshold at $\mathrm{C}_{f}$ in SGNs displaying a chopper discharge pattern (periodically alternating phases of high firing rates with low firing rates, typical of multipolar cells in the posterior ventral cochlear nucleus), showed that the differences in rate faded away opposite to those shown at the level of the auditory nerve. Peak rates were still significantly lower in $\operatorname{RBE}^{\mathrm{KO} / \mathrm{KO}}(n=29 \mathrm{SGNs}, \mathrm{N}=9)$ as compared to $\operatorname{RBE}^{\mathrm{WT} / W T}$ ( $n=16 \mathrm{SGNs}, \mathrm{N}=9$ ) mice ( $p=0.0303$, Mann-Whitney-Wilcoxon test), while the adapted rate showed a non-significant trend towards reduction in $\operatorname{RBE}^{K O / K O}\left(p=0.0538\right.$, t-test). Box plots show $10,25,50,75$ and $90^{\text {th }}$ percentiles and means are shown as crosses, as for (B). (B) Same recordings paradigm as (A) performed in a fiber with bushy cell discharge pattern (similar to the one found in SGNs, typical also in this type of cochlear nucleus neurons) showed comparable responses in both peak ( $p=0.2601$, Mann-Whitney-Wilcoxon test) and adapted rate $\left(p=0.0510\right.$, Mann-Whitney-Wilcoxon test) in $\operatorname{RBE}^{\mathrm{KO} / \mathrm{KO}}(n=19 \mathrm{SGNs}, \mathrm{N}=7)$ and RBEWT/WT $(n=28 \mathrm{SGNs}, \mathrm{N}=10)$ DOI: https://doi.org/10.7554/eLife.29275.017 


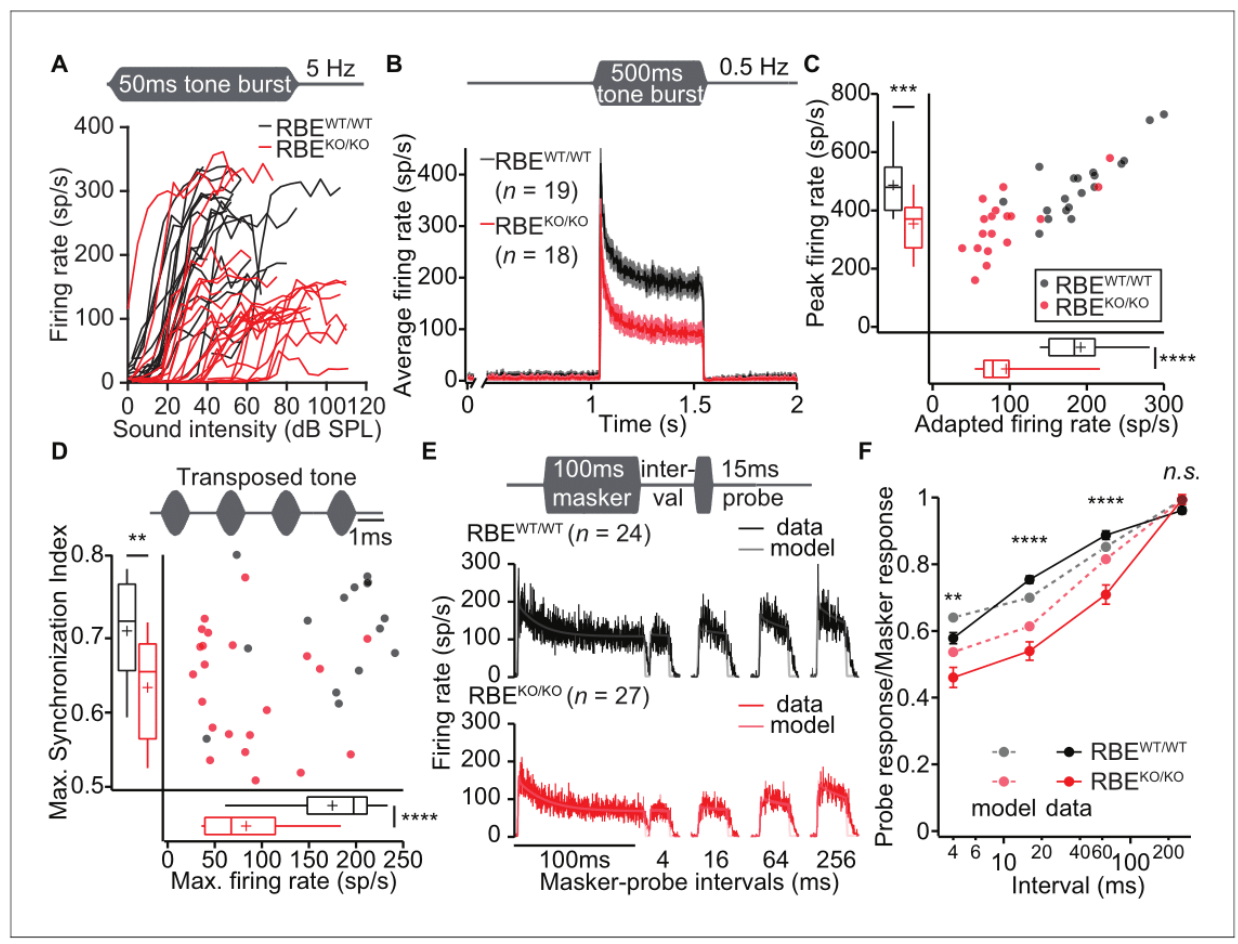

Figure 11. Lack of ribbons impairs vesicle replenishment in $\mathrm{RBE}^{\mathrm{KO} / \mathrm{KO}}$ mice. (A) Intensity coding was largely preserved for suprathreshold sound stimulation: rate-level functions (average increase in spike rate with stimulus intensity) of SGNs in response to 50 ms tone bursts at $\mathrm{C}_{\mathrm{f}}$ at $5 \mathrm{~Hz}$ corroborated the notion of increased sound threshold but were otherwise comparable in RBE ${ }^{\mathrm{KO} / \mathrm{KO}}\left(n=24 \mathrm{SGNs}, \mathrm{N}=8\right.$ ) and RBE ${ }^{\mathrm{WT}} / \mathrm{WT}$ ( $n=19 \mathrm{SGNs}$, $\mathrm{N}=7$ ) mice, for quantification see Figure 11-figure supplement $1 \mathrm{C}$. (B) Average PSTH recorded in response to $50 \times 500 \mathrm{~ms}$ tone bursts at $\mathrm{C}_{\mathrm{f}}$ and 30 $\mathrm{dB}$ above threshold at a stimulus rate of $0.5 \mathrm{~Hz}$ (bin width $=2 \mathrm{~ms}$ ): the onset response to this stimulus was preserved better in $\mathrm{RBE}{ }^{\mathrm{KO} / \mathrm{KO}} \mathrm{SGNs}$ ( $n=18$ $\mathrm{SGNs}, \mathrm{N}=7$ ) as compared to higher stimulation rates (Figure 9), but it was still lower than in RBEWT/WT $\mathrm{SGNs}(n=19 \mathrm{SGNs}, \mathrm{N}=7)$, as well as the adapted firing rate. Mean (line) \pm S.E.M. (shaded areas) are displayed. (C) Scatterplot of peak (highest 2 ms bin) and adapted (averaged $405-415$ ms from response onset) firing rates: significantly reduced peak ( $p=0.0005$, t-test) and adapted ( $<<0.0001$, Mann-Whitney-Wilcoxon test) rates in RBE ${ }^{K O / K O}$ SGNs. Box plots show 10, 25, 50, 75 and $90^{\text {th }}$ percentiles and means are shown as crosses, as for (D). (D) Temporal precision and reliability of sound coding is impaired in $\mathrm{RBE}^{\mathrm{KO} / \mathrm{KO}} \mathrm{SGNs}$. Synchronization of firing to stimulus and firing rates (reflecting spike probability) were reduced when probed with amplitude-modulated (transposed) tones (continuous stimulation with a carrier frequency at $\mathrm{C}_{\mathrm{f}}$ and at a modulation frequency of $500 \mathrm{~Hz}$ ) $\left(p=0.0043\right.$, t-test, for synchronization index, and $p<0.0001$, t-test, for firing rate, when comparing RBE ${ }^{\mathrm{KO} / \mathrm{KO}}(n=22 \mathrm{SGNs}, \mathrm{N}=7)$ and RBE ${ }^{\mathrm{WT} / \mathrm{WT}}$ ( $n=15$ $\mathrm{SGNs}, \mathrm{N}=6$ 6) $\mathrm{SGNs}$ ). (E) Forward masking experiments were used to study presynaptic SV dynamics: a stimulus complex consisting of a 100 ms masker stimulus, a silent interval of 4/16/64/256 ms and a $15 \mathrm{~ms}$ probe (both at the characteristic frequency, $30 \mathrm{~dB}$ above threshold) was presented at $2 \mathrm{~Hz}$. The averaged data after alignment of RBE ${ }^{\mathrm{WT} / W T}$ ( $n=24 \mathrm{SGNs}, \mathrm{N}=7$, black) and RBE ${ }^{\mathrm{KO} / \mathrm{KO}}$ ( $n=27 \mathrm{SGNs}, \mathrm{N}=8$, red) SGNs responses are displayed as described in Materials and methods. On top of the data we present a fit of a biophysical model (light gray and pink lines) to the data used to study the SV dynamics at the AZ. The refilling and fusion rate constant during spontaneous and stimulated conditions as well as the number of occupied release sites are provided in Table 2. (F) Recovery of onset response showed as ratio of probe and masker response (number of spikes during first 10 ms, mean \pm S.E.M), and prediction (dashed lines) derived from the model fit shown in (E). Recovery was slower in $\mathrm{RBE}{ }^{\mathrm{KO} / \mathrm{KO}} \mathrm{SGNs}(n=27 \mathrm{SGNs}, \mathrm{N}=8$ ) as compared to RBE ${ }^{W T} / W_{T}(n=24 S G N s, N=7$ ) with significant differences in the ratio after $4 \mathrm{~ms}(p=0.0019, t$-test), 16 ms ( $p<0.0001$, Mann-WhitneyWilcoxon test), and 64 ms masker-probe intervals ( $p<0.0001$, Mann-Whitney-Wilcoxon test), but not after $256 \mathrm{~ms}$ ( $p=0.0835$, t-test). DOl: https://doi.org/10.7554/eLife.29275.019

The following figure supplement is available for figure 11

Figure supplement 1. Rate-level functions and dynamic range remained unchanged in $\mathrm{RBE}^{\mathrm{KO} / \mathrm{KO}}$ DOI: https://doi.org/10.7554/eLife.29275.020

seen with shorter inter-stimulus interval (e.g. 200 ms, Figure 9C), likely reflecting more complete SV replenishment (i.e. larger standing RRP) owing to the longer recovery interval ( $2 \mathrm{~s}$ vs. $200 \mathrm{~ms}$ ). However, the adapted spike rate of RBE ${ }^{\mathrm{KO} / K O} \mathrm{SGNs}$ was even more reduced than found with $50 \mathrm{~ms}$ tone bursts (to about half of that for RBE ${ }^{\mathrm{WT}} / \mathrm{WT}$ SGNs, Figure 11B,C) highlighting the impaired SV 
Table 1. Average double-exponential fitting results to peristimulus time histograms obtained by $50 \mathrm{~ms}$ tone bursts $30 \mathrm{~dB}$ above threshold at $\mathrm{C}_{\mathrm{f}}(200 \mathrm{~ms}$ inter-stimulus interval).

\begin{tabular}{lllll} 
& Fast time constant & Slow time constant & Amplitude fast component & Amplitude slow component \\
\hline RBE $^{\text {WT/WT }}$ & $6.31 \pm 0.77$ & $95.63 \pm 24.21$ & $484.61 \pm 104.76$ & $58.42 \pm 12.90$ \\
\hline RBE $^{\text {KO/KO }}$ & $18.79 \pm 6.91$ & $101.66 \pm 27.77$ & $310.56 \pm 73.60$ & $64.92 \pm 107.49$ \\
\hline p-value & 0.0045 & 0.3580 & 0.0519 & 0.9475
\end{tabular}

DOl: https://doi.org/10.7554/eLife.29275.018

replenishment during prolonged stimulation. Finally, we evaluated presynaptic vesicle pool dynamics by recording and modeling responses to forward masking protocols (Harris and Dallos, 1979), that are thought to reflect depletion and recovery of the RRP (Figure 11E,F; Figure 11-figure supplement $1 \mathrm{C}$ ). We approximated the recovery from forward masking by single exponential fitting (Figure 11-figure supplement 1C) to provide an estimate of the kinetics of vesicle poo replenishment. The time constant of recovery was prolonged in RBE ${ }^{K O} / \mathrm{KO} S G N s(90.80 \pm 8.66 \mathrm{~ms}, \mathrm{~S}$. D. $=45.00 \mathrm{~ms}, n=27 \mathrm{SGNs}, \mathrm{N}=8$ vs. $33.53 \pm 5.74 \mathrm{~ms}, \mathrm{~S} . \mathrm{D} .=28.11 \mathrm{~ms}$, in RBEWT/WT $\mathrm{SGNs}, n=24$ SGNs, $N=7, p<0.0001$, Mann-Whitney-Wilcoxon test) indicating slowed RRP replenishment in the absence of the ribbon. We noted that SGNs showed considerably lower spontaneous and evoked rates during the forward masking paradigm compared to other stimulus protocols (compare Figure 11B E), likely due to enhanced RRP depletion with the more extended stimulation in this protocol.

Amplitude and waveform of the forward masking responses were consistent with a two-fold reduction of the number of contributing vesicular release sites of the RRP ( ${ }^{\prime}{ }_{\text {slot }}$ : contributing release sites during forward masking, $\mathrm{N}_{\text {slot }}$ : the contributing release sites for the same $\mathrm{AZs}$ during tone bursts at $5 \mathrm{~Hz}$ stimulation). The ratio $\mathrm{N}_{\text {slots }} / \mathrm{N}^{\prime}$ slots was estimated to be 2.2 for $\mathrm{RBE}^{\mathrm{KO} / \mathrm{KO}}$ and 2.3 for $\mathrm{RBE}^{\mathrm{WT} / \mathrm{WT}}$ from the drop in spontaneous and evoked SGN spiking rates. We used a previously developed biophysical model of RRP dynamics and spike generation (Frank et al., 2010; Jung et al., $2015 b)$ to extract information on fusion and replenishment rate constants as well as the $\mathrm{N}_{\text {slot }}$ by fitting the responses to tone bursts, that is PSTHs with 100, 200 and 2000 ms inter-stimulus interval, and also the forward masking spiking data across all recovery intervals (Figure 11E). Importantly, only the first $50 \mathrm{~ms}$ of the $500 \mathrm{~ms}$ stimulus response during the PSTH 2000 were included in the fit; any later adaptation processes were disregarded, as they were not accounted by the model's equations.

The results of model fitting suggested that during the forward masking only about half of all release sites $\left(\mathrm{N}_{\text {slots }}\right)$ were engaged in the response $\left(\mathrm{N}^{\prime}{ }_{\text {slots }}\right)$. Throughout, RBE ${ }^{\mathrm{KO} / \mathrm{KO}} \mathrm{SGNs}$ showed a lower fusion rate than the RBE ${ }^{\mathrm{WT} / \mathrm{WT}} \mathrm{SGNs}$, reflecting the reduced onset response in $\mathrm{RBE}^{\mathrm{KO} / \mathrm{KO}}$ SGNs. When more recovery time was allowed, that is in the $0.5 \mathrm{~Hz}$ tone burst and the forward masking, where recovery times from around 250 to $500 \mathrm{~ms}$ occured between the probe and subsequent masker, the onset response improved in $\mathrm{RBE}^{\mathrm{KO} / \mathrm{KO}}$. Consequently, the estimated fusion rate almost reached the level of RBE ${ }^{W T / W T}$ in the forward masking fits. With the scaling factors of approximately 2 , the estimates for the number of release sites were consistent between tone bursts and forward

Table 2. Parameters for the biophysical model capturing the release dynamics during forwardmasking and repetitive tone burst experiments

\begin{tabular}{|c|c|c|c|c|}
\hline & \multicolumn{2}{|c|}{ Forward masking } & \multicolumn{2}{|c|}{ All tone bursts - global fit } \\
\hline & $\mathrm{RBE}^{\mathrm{WT} / \mathrm{WT}}$ & $\mathrm{RBE}^{\mathrm{KO} / \mathrm{KO}}$ & $\mathrm{RBE}^{\mathrm{WT} / \mathrm{WT}}$ & $\mathrm{RBE}^{\mathrm{KO} / \mathrm{KO}}$ \\
\hline $\mathrm{N}_{\text {slots }}$ & 8 & 6.5 & 15.6 & 13.7 \\
\hline $\mathrm{k}_{\text {refill, stim }}$ & 29.5 & 18.2 & 18.4 & 19.9 \\
\hline$k_{\text {refill, spont }}$ & 13.3 & 7.1 & 5.59 & 2.71 \\
\hline$k_{\text {fusion, stim }}$ & 32.2 & 31 & 82.5 & 45.1 \\
\hline$k_{\text {fusion, spont }}$ & 0.6 & 0.9 & 0.63 & 0.54 \\
\hline
\end{tabular}

DOI: https://doi.org/10.7554/eLife.29275.021 
masking data, and in both cases only slightly smaller for the RBE ${ }^{\mathrm{KO} / \mathrm{KO}}$ (Table 2). Taken together the fits from forward masking and tone bursts suggest that the total number of release sites (RRP) was only slightly reduced at ribbonless synapses of $\mathrm{RBE}^{\mathrm{KO} / \mathrm{KO}} \mathrm{IHCs}$. However, a strong firing response at sound onset, that is release of a large standing RRP, required longer recovery indicating more efficient $\mathrm{SV}$ replenishment in the presence of ribbons, which is reflected in the larger refilling rate constants estimated by the model of RBE ${ }^{\mathrm{WT} / \mathrm{WT}}$ synapses.

\section{Discussion}

The role of the synaptic ribbon has remained a topic of intense research. Here, we studied the structure and function of IHC afferent synapses with SGNs in mice lacking RIBEYE, the core component of the synaptic ribbon. Morphologically, synapses of RBE ${ }^{\mathrm{KO} / K O} \mathrm{HC}$ did not simply lack ribbons but instead appeared transformed to contacts where release from multiple AZ feeds into one postsynaptic bouton. Synaptic transmission was impaired at the ribbonless IHC synapses of RBE ${ }^{\mathrm{KO} / K O}$ mice. Spontaneous SGN firing was reduced, sound-evoked firing had higher sound thresholds, lower peak and adapted rates, recovered more slowly from adaptation and had a greater temporal jitter. Modelling of synaptic sound encoding corroborated the notion of reduced rates of SV fusion and replenishment at the ribbonless synapses of $\mathrm{RBE}^{\mathrm{KO} / \mathrm{KO}}$ mice. Analysis of $\mathrm{IHC}$ function revealed a small depolarized shift in the operating range of the synaptic $\mathrm{Ca}_{v} 1.3 \mathrm{Ca}^{2+}$-channels, which likely contributes to the reduced spontaneous and evoked firing rates and to the elevated sound thresholds of $\mathrm{RBE}^{\mathrm{KO} / \mathrm{KO}} \mathrm{SGNs} . \Delta \mathrm{C}_{\mathrm{m}}$ recordings revealed a mild reduction of exocytosis but only for weaker depolarizations, which we primarily attribute to the depolarized shift of $\mathrm{Ca}^{2+}$-channel activation. In summary, our data support a role of the ribbon in vesicle replenishment and $\mathrm{Ca}^{2+}$-channel regulation at the AZs as required for synchronous activation of SGNs in normal hearing. However, our analysis of $\mathrm{RBE}^{\mathrm{KO} / \mathrm{KO}}$ mice likely underestimated the role of the ribbon due to substantial compensation that is best illustrated by the striking transformation of $A Z$ morphology and the mild ex vivo phenotype of $\mathrm{IHC} \mathrm{Ca}{ }^{2+}$-influx and exocytosis.

\section{RIBEYE deletion transforms IHC synapses to 'conventional-like' presynaptic ultrastructure, where multiple ribbonless AZs collectively maintain large complements of $\mathrm{Ca}^{2+}$-channels and SVs}

Our work confirms the central role of RIBEYE for forming synaptic ribbons (Schmitz et al., 2000; Magupalli et al., 2008; Maxeiner et al., 2016). We did not observe structures reminiscent of 'ghost ribbons' reported for ribeye mutants in zebrafish neuromast hair cells (Lv et al., 2016) in IHCs of $\mathrm{RBE}^{\mathrm{KO} / \mathrm{KO}}$ mice. These ghost ribbons were characterized as a halo of synaptic vesicles around a nonelectron-dense area that resembled in size, though smaller, and shape to a synaptic ribbon. In zebrafish, two gene copies of ribeye (ribeye $a$ and b) exist, making it harder to achieve a complete knockout (Lv et al., 2016; Van Epps et al., 2004). In keeping with this notion, Lv et al. found residual immunofluorescence of ribeye $a$ in the double mutants. Hence, we speculate that residual RIBEYE, possibly together with other scaffold proteins such as piccolo, might have formed the observed electron-translucent SV-framed structures (Lv et al., 2016). In contrast, immunofluorescence, as well as electron microscopy, revealed the complete absence of RIBEYE and ribbons in IHCs of RBE ${ }^{\mathrm{KO} / K O}$ mice in our work and the companion study (Becker et al.), which is in agreement with findings in the $\mathrm{RBE}^{\mathrm{KO} / \mathrm{KO}}$ mouse retina (Maxeiner et al., 2016).

IHC synapses normally employ a single ribbon-type AZ. But in the absence of RIBEYE, there were typically two or more ribbonless AZs, akin to multiple conventional AZs (Figure 2). These ribbonless 'conventional' $\mathrm{AZs}$ at RBE ${ }^{\mathrm{KO} / \mathrm{KO}} \mathrm{IHC}$ synapses consist mostly of roundish PDs, each with a cluster of $\mathrm{SV}$, of which approximately one third were directly adjacent to the plasma membrane (membraneproximal: MP-SVs). Using electron tomography we found that about two-thirds of the MP-SVs were tethered to the AZ membrane, which was comparable to RBE ${ }^{\mathrm{WT} / W T}$ AZs (Figure 3). We speculate that $\mathrm{SV}$ s associated with the PD, but not facing the membrane (PDA-SVs), serve to replenish the release sites once tethered MP-SVs fused, and that the ribbonless PD more likely acts in long-range $\mathrm{SV}$ tethering to the $\mathrm{AZ}$ in analogy to what is considered for conventional AZs (Cole et al., 2016; Fernández-Busnadiego et al., 2013; Siksou et al., 2007). We assume that absence of RIBEYE does not alter SV size since electron tomography, which provides the most reliable estimation of SV size, did not reveal differences in SV diameter between $\mathrm{RBE}^{\mathrm{KO} / \mathrm{KO}}$ and $\mathrm{RBE}{ }^{\mathrm{WT}} \mathrm{WT} A \mathrm{As}$, at least when 
considering all SVs. The RBE ${ }^{\mathrm{KO} / \mathrm{KO}} \mathrm{PDs}$, like in $\mathrm{RBE}^{\mathrm{WT} / \mathrm{WT}}$, contained bassoon, Cav1.3, and RIM2, but lacked piccolino which is likely part of the ribbon in RBE ${ }^{\mathrm{WT}} / \mathrm{WT}$ (Figure 1) (Dick et al., 2001; Khimich et al., 2005; Limbach et al., 2011; Regus-Leidig et al., 2013).

To some extent, the multi-AZ morphology is reminiscent of IHC synapses prior to synaptic maturation (Huang et al., 2012; Sendin et al., 2007; Sobkowicz et al., 1982; Wong et al., 2014). While we cannot rule out some sort of developmental delay of RIBEYE-deficient IHCs, we suspect that the morphological transformation into a multi-AZ morphology reflects a compensatory effort. Reasons for our interpretation include (i) the same morphological phenotype of RBE ${ }^{\mathrm{KO} / \mathrm{KO}} \mathrm{IHCs}$ at 8 months of age (Figure 2), (ii) the finding of highly regular PDs at RBE ${ }^{\mathrm{KO} / \mathrm{KO}} \mathrm{IHC}$ s synapses, which differs from less well-defined PDs at immature AZs (Wong et al., 2014), (iii) the typical continuous and large PSD of $\mathrm{RBE}^{\mathrm{KO} / \mathrm{KO}} \mathrm{IHC}$ s synapses (Figure 2, see also the accompanying paper by Becker et al.) as a characteristic of a mature synapse, rather than the several smaller PSD patches at developing IHC synapses (Wong et al., 2014), and (iv) the synaptically confined Cav1.3 $\mathrm{Ca}^{2+}$-channel clusters, normal amplitude of $\mathrm{IHC} \mathrm{I}_{\mathrm{Ca}}$ and mature amplitude of synaptic $\mathrm{Ca}^{2+}$-signals, rather than massive extrasynaptic Cav1.3 abundance and larger whole-cell $I_{C a}$ but smaller synaptic $\mathrm{Ca}^{2+}$-signals in immature IHCs (Wong et al., 2014; Zampini et al., 2010). The multi-AZ morphology of the RBE ${ }^{\mathrm{KO} / K O} \mathrm{IHC}$ synapses was also corroborated by high- and super-resolution microscopy of bassoon and Cav1.3 immunofluorescence (Figure 4). The organization in several smaller $\mathrm{Ca}^{2+}$-channel clusters likely explains the

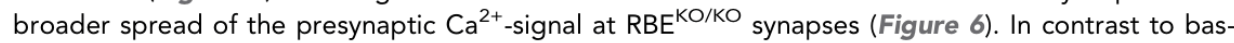
soon mutant mice (Frank et al., 2010; Jing et al., 2013), the number of synaptic $\mathrm{Ca}^{2+}$-channels was not reduced in $\mathrm{RBE}{ }^{\mathrm{KO} / \mathrm{KO}}$ mice as shown by normal amplitudes of whole-cell $\mathrm{I}_{\mathrm{Ca}}$ and synaptic $\mathrm{Ca}^{2+}$. signals. Therefore, the loss of synaptic $\mathrm{Ca}^{2+}$-channels from the bassoon-deficient ribbonless IHC synapses, indicates a role of bassoon in promoting $\mathrm{Ca}^{2+}$-channel tethering at the $\mathrm{AZ}$ likely via interaction with RIM-binding protein (Davydova et al., 2014), which was previously shown to interact with Cav1.3 $\mathrm{Ca}^{2+}$-channels (Hibino et al., 2002) and is required for establishing a normal $\mathrm{Ca}^{2+}$-channel complement of the IHC AZ (Krinner et al., 2017).

Interestingly, we observed changes in $\mathrm{Ca}^{2+}$-channel function in $\mathrm{RBE}^{\mathrm{KO} / \mathrm{KO}} \| \mathrm{HCs}$ : the voltagedependence of $\mathrm{Ca}^{2+}$-channel activation was slightly, but significantly, shifted to more depolarized potentials both at the levels of whole-cell $\mathrm{Ca}^{2+}$-current $\left(\mathrm{V}_{\mathrm{h}}+2 \mathrm{mV}\right)$ and synaptic $\mathrm{Ca}^{2+}$-influx at individual synapses $\left(\mathrm{V}_{\mathrm{h}}+5 \mathrm{mV}\right.$ ) (at $5 \mathrm{mM}\left[\mathrm{Ca}^{2+}\right]_{\mathrm{e}}$, Figure 5 and 6). Similar as in this study, an enhanced inactivation (Figure 5) of $\mathrm{I}_{\mathrm{Ca}}$ was also found in bassoon-deficient IHCs, while their $\mathrm{V}_{\mathrm{h}}$ was actually mildly shifted in the opposite direction $\left(-3 \mathrm{mV}\right.$ for imaging of synaptic $\left.\mathrm{Ca}^{2+}\right)$ and unaltered at the level of the whole-cell $I_{\mathrm{Ca}}$ (Frank et al., 2010). One potential reason for why the depolarized $V_{\mathrm{h} \text {-shift }}$ of the synaptic $\mathrm{Ca}^{2+}$-influx was greater than that of the whole-cell $\mathrm{Ca}^{2+}$-influx is the contribution of extrasynaptic $\mathrm{Ca}^{2+}$-channels to the whole-cell $\mathrm{Ca}^{2+}$-influx. They are thought to contribute approxi-

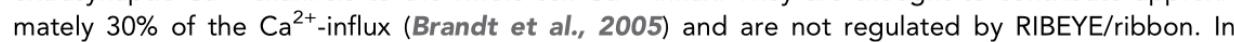
order to test whether the depolarized $\mathrm{V}_{\mathrm{h}}$-shift of synaptic $\mathrm{Ca}^{2+}$ translates into changes in transmitter release, we recorded exocytic $\Delta \mathrm{C}_{\mathrm{m}}$ for different depolarization potentials. A small, but significant reduction of exocytosis for weak depolarizations in $\mathrm{RBE}^{\mathrm{KO} / \mathrm{KO}} \mathrm{IHCs}$ (Figure 7, seen also in the accompanying paper by Becker et al.) suggests that the $V_{h}$ shift is relevant for hair cell transmission (see also below). How enhanced $\mathrm{I}_{\mathrm{Ca}}$ inactivation might affect sound encoding is addressed by work on $\mathrm{Ca}^{2+}$-binding proteins $(\mathrm{CaBPs})$ that are thought to antagonize calmodulin's role in mediating $\mathrm{I}_{\mathrm{Ca}}$ inactivation (Lee et al., 1999; Peterson et al., 1999). Among the several CaBPs expressed in IHCs (Cui et al., 2007; Picher et al., 2017; Schrauwen et al., 2012; Yang et al., 2006), CaBP2 is defective in human genetic hearing loss DFNB93 (Picher et al., 2017; Schrauwen et al., 2012) and required for hearing likely via inhibition of $\mathrm{IHC} \mathrm{I}_{\mathrm{Ca}}$ inactivation (Picher et al., 2017). However, deletion of $\mathrm{CaBP} 4$ in mice caused only a very mild increase of $\mathrm{I}_{\mathrm{Ca}}$ inactivation similar to the one found here and did not alter auditory brainstem responses (Cui et al., 2007). Future studies need to address how RIBEYE/ribbons mechanistically regulate the function and spatial organization of $\mathrm{Ca}^{2+}$. channels.

\section{What can the RIBEYE knock-out tell us about the function of the ribbon in sensory coding?}

Over some decades, research on retinal photoreceptors and bipolar cells, on hair cells of the inner ear and the lateral lines, on electroreceptors as well as pineal cells, has aimed to elucidate the function(s) of the synaptic ribbon. Current hypotheses state that the ribbon functions in (i) replenishing 
release sites ([Bunt, 1971; Frank et al., 2010; von Gersdorff et al., 1996; Lenzi et al., 2002; Maxeiner et al., 2016; Snellman et al., 2011; Vaithianathan and Matthews, 2014] for a deviating view see \{Jackman et al., 2009]), potentially by facilitated diffusion of SVs on the ribbon surface towards the site of consumption (Graydon et al., 2014) and SV priming (Grabner and Zenisek, 2013; Snellman et al., 2011), (ii) establishing a large complement of vesicular release sites and $\mathrm{Ca}^{2+}$-channels at the active zone (Frank et al., 2010; Khimich et al., 2005), which remained hard to disentangle from potential function of bassoon (Frank et al., 2010; Jing et al., 2013), (iii) ensuring close spatial coupling of $\mathrm{Ca}^{2+}$-channels and vesicular release sites (Maxeiner et al., 2016) or enhancing presynaptic $\mathrm{Ca}^{2+}$-signals by limiting diffusional $\mathrm{Ca}^{2+}$-spread (Graydon et al., 2011), (iv) contributing to multivesicular release (Graydon et al., 2011; Jing et al., 2013; Mehta et al., 2013), and (v) contributing to SV reformation from endocytosed membranes (Jung et al., 2015b; Khimich et al., 2005; Schwarz et al., 2011). Clearly, SV-replenishment was impaired at the ribbon-less IHC synapses of RBE ${ }^{\mathrm{KO} / \mathrm{KO}}$ mice. This is shown by slowed recovery from forward-masking and the use-dependent reduction of peak and adapted firing rates, which we further scrutinized by modeling. Therefore, our study supports a role of the ribbon in vesicle replenishment, which is also found in the accompanying paper by Becker et al.. Why RRP-recovery was not significantly altered when probed with pairs $20 \mathrm{~ms}$ long maximal $\mathrm{Ca}^{2+}$-influx by membrane capacitance measurements in $\mathrm{HHCs}$ (Figure 7 ) will need to be addressed in future studies, ideally using paired pre-and postsynaptic recordings of synaptic transmission with depolarizations of varying strength.

Each of the ribbon-manipulations employed to analyze its role has strengths, but also weaknesses, such as changes in other AZ proteins and long-term compensatory processes (e.g. bassoon deletion), complex manipulations (e.g. diurnal changes or hibernation: [Hull et al., 2006; Mehta et al., 2013; Spiwoks-Becker et al., 2004]) and photoablation (Mehta et al., 2013; Snellman et al., 2011). Genetic RIBEYE manipulations (Lv et al., 2016; Maxeiner et al., 2016; Sheets et al., 2011; Van Epps et al., 2004) have the greatest molecular specificity, but in some cases, were incomplete, and to some extent masked by compensation. In fact, our study of IHCs, unlike the situation for bipolar cell retinal ribbons (Maxeiner et al., 2016), suggests that some features of the IHC ribbon-type $\mathrm{AZ}$ can be very well replaced by a ribbonless multi-AZ morphology: the synaptic complement of $\mathrm{Ca}^{2+}$-channels and $\mathrm{SV}$ s, as well as exocytic $\Delta \mathrm{C}_{\mathrm{m}}$ elicited by strong depolarizations, were similar. Therefore, we likely underestimated the role of the ribbon in sound encoding in our present study.

For sound encoding at the afferent synapses between IHCs and SGNs, we observed some commonalities and differences with the bassoon mutants and $\mathrm{RBE}^{\mathrm{KO} / \mathrm{KO}}$ mice, whereby the stronger phenotype of bassoon mutants suggests additive effects of bassoon and ribbon loss. Recordings from single SGNs indicate reduced peak and adapted release rates at the IHC synapses of ribbonless synapses, as well as impaired temporal precision of coding. High temporal precision is a hallmark of synaptic sound encoding (e.g. (Köppl, 1997). Reduced release rates or smaller EPSC sizes would increase the temporal jitter (Buran et al., 2010; Li et al., 2014; Rutherford et al., 2012; Wittig and Parsons, 2008). Reduced spike rates and increased jitter of release likely explain the reduced ABR wave I amplitude in both mutants. A striking difference from bassoon mutants, however, is that sound encoding in $R B E^{K O / K O}$ mice was impaired substantially, despite unaltered exocytic $\Delta C_{m}$ upon strong stimulation. We propose two mechanisms with likely additive effects to explain this surprising finding: i) the small depolarized shift of synaptic $\mathrm{Ca}^{2+}$-channels might contribute the lower spontaneous and evoked firing rates as well as higher sound thresholds of SGNs and ii) the reduced SVreplenishment might not suffice to balance the rate of consumption leading to a smaller standing RRP in vivo, while the arrest of exocytosis in the voltage-clamped IHCs for tens of seconds likely enables complete filling of the release sites (max. standing RRP). The changes in $\mathrm{Ca}^{2+}$-channel gating observed in $\mathrm{IHC}$ s of $\mathrm{RBE}{ }^{\mathrm{KO} / \mathrm{KO}}$ mice were unexpected, as so far, a direct or indirect interaction of RIBEYE and $\mathrm{Ca}^{2+}$-channels have not been described. Clearly, future studies, including studies on the potential regulation of $\mathrm{Cav}_{\mathrm{v}} 1.3 \mathrm{Ca}^{2+}$-channels by RIBEYE and piccolino in heterologous expression systems, paired pre- and postsynaptic recordings, as well as further computational modeling, are required. 


\section{Materials and methods}

Key resources table

\begin{tabular}{|c|c|c|c|c|}
\hline $\begin{array}{l}\text { Reagent type (species) } \\
\text { or resource }\end{array}$ & Designation & Source or reference & Identifiers & Additional information \\
\hline $\begin{array}{l}\text { Strain, strain background } \\
\text { (Mus musculus) }\end{array}$ & $\begin{array}{l}\text { Constitutive RIBEYE knockout, } \\
\text { C57BL/6 background }\end{array}$ & PMID: 26929012 & & \\
\hline Antibody & CtBP2 (mouse monoclonal) & BD Biosciences & 612044 & $1: 200$ \\
\hline Antibody & PSD-95 (mouse monoclonal) & Sigma Aldrich & P246-100ul & $1: 200$ \\
\hline Antibody & $\begin{array}{l}\text { Bassoon SAP7f407 } \\
\text { (mouse monoclonal) }\end{array}$ & Abcam & ab82958 & $1: 200$ \\
\hline Antibody & Bassoon (guinea pig polyclonal) & Synaptic Systems & 141004 & $1: 500$ \\
\hline Antibody & RIM2 (rabbit polyclonal) & Synaptic Systems & 140103 & $1: 100$ \\
\hline Antibody & $\mathrm{Ca}_{\mathrm{v}} 1.3$ (rabbit polyclonal) & Alomone Labs & ACC 005 & $\begin{array}{l}1: 75 \\
\text { or } 1: 100\end{array}$ \\
\hline Antibody & Piccolino (rabbit polyclonal) & Regus-Leidig et al. (2013) & & $1: 500$ \\
\hline Antibody & $\begin{array}{l}\text { Parvalbumin } \alpha \\
\text { (guinea pig polyclonal) }\end{array}$ & Synaptic Systems & 195004 & $1: 1000$ \\
\hline Antibody & $\begin{array}{l}\text { Calbindin } 28 \mathrm{k} \\
\text { (mouse monoclonal) }\end{array}$ & Swant & $07(F)$ & $1: 500$ \\
\hline Antibody & Calretinin (rabbit polyclonal) & Swant & $1893-0114$ & $1: 1000$ \\
\hline Antibody & $\begin{array}{l}\text { Alexa Fluor } 488 \text { conjugated } \\
\text { anti-rabbit (goat polyclonal) }\end{array}$ & Invitrogen & A11008 & $1: 200$ \\
\hline Antibody & $\begin{array}{l}\text { Alexa Fluor } 488 \text { conjugated } \\
\text { anti-guinea-pig (goat polyclonal) }\end{array}$ & Invitrogen & A11004 & $1: 200$ \\
\hline Antibody & $\begin{array}{l}\text { Alexa Fluor } 568 \text { conjugated } \\
\text { anti-mouse (goat polyclonal) }\end{array}$ & Invitrogen & A11073 & $1: 200$ \\
\hline Antibody & $\begin{array}{l}\text { Alexa Fluor } 647 \text { conjugated } \\
\text { anti-rabbit (donkey polyclonal) }\end{array}$ & Invitrogen & A31573 & $1: 200$ \\
\hline Antibody & $\begin{array}{l}\text { STAR580 conjugated } \\
\text { anti-mouse (goat polyclonal) }\end{array}$ & Abberior & $2-0002-005-1$ & $1: 200$ \\
\hline Antibody & $\begin{array}{l}\text { STAR580 conjugated } \\
\text { anti-rabbit (goat polyclonal) }\end{array}$ & Abberior & $2-0012-005-8$ & $1: 200$ \\
\hline Antibody & $\begin{array}{l}\text { STAR635p conjugated } \\
\text { anti-mouse (goat polyclonal) }\end{array}$ & Abberior & $2-0002-007-5$ & $1: 200$ \\
\hline Antibody & $\begin{array}{l}\text { STAR635p conjugated } \\
\text { anti-rabbit (goat polyclonal) }\end{array}$ & Abberior & $2-0012-007-2$ & $1: 200$ \\
\hline Software, algorithm & Patchmaster or Pulse & $\begin{array}{l}\text { http://www.heka.com/products/ } \\
\text { products_main.html\#soft_pm }\end{array}$ & RRID:SCR_000034 & \\
\hline Software, algorithm & IGOR Pro & $\begin{array}{l}\text { http://www.wavemetrics.com/products } \\
\text { /igorpro/igorpro.htm }\end{array}$ & RRID:SCR_000325 & \\
\hline Software, algorithm & Patchers Power Tools & $\begin{array}{l}\text { http://www3.mpibpc.mpg.de/groups } \\
\text { /neher/index.php?page=software }\end{array}$ & RRID:SCR_001950 & \\
\hline Software, algorithm & MATLAB & $\begin{array}{l}\text { http://www.mathworks.com/ } \\
\text { products/matlab/ }\end{array}$ & RRID:SCR_001622 & \\
\hline Software, algorithm & Gatan Microscopy Suite & $\begin{array}{l}\text { http://www.gatan.com/products/tem } \\
\text {-analysis/gatan-microscopy-suite-software }\end{array}$ & RRID:SCR_014492 & $\begin{array}{l}\text { DigitalMicrograph } \\
\text { scripting }\end{array}$ \\
\hline Software, algorithm & Reconstruct & PMID: 15817063 & & \\
\hline Software, algorithm & Serial-EM & PMID: 16182563 & & \\
\hline Software, algorithm & $3 \mathrm{dmod}$ & PMID: 8742726 & & \\
\hline Software, algorithm & IMOD & http://bio3d.colorado.edu/imod & RRID:SCR_003297 & \\
\hline Software, algorithm & Genetic fit algorithm & Sanchez del Rio and Pareschi, 2001 & doi: $10.1117 / 12.411624$ & \\
\hline Software, algorithm & Fiji & http://fiji.sc & RRID:SCR_002285 & \\
\hline
\end{tabular}

Jean et al. eLife 2018;7:e29275. DOI: https://doi.org/10.7554/eLife.29275 
Continued

\begin{tabular}{|c|c|c|c|c|}
\hline $\begin{array}{l}\text { Reagent type (species) } \\
\text { or resource }\end{array}$ & Designation & Source or reference & Identifiers & Additional information \\
\hline Software, algorithm & ImageJ & https://imagej.nih.gov/ij/ & RRID:SCR_003070 & \\
\hline Software, algorithm & Imaris & http://www.bitplane.com/imaris/imaris & RRID:SCR_007370 & \\
\hline Software, algorithm & Excel & microsoft.com/mac/excel & & \\
\hline Software, algorithm & Origin & $\begin{array}{l}\text { http://www.originlab.com/index. } \\
\text { aspx?go=PRODUCTS/Origin }\end{array}$ & RRID:SCR_014212 & \\
\hline Software, algorithm & GraphPad Prism & $\begin{array}{l}\text { https://www.graphpad.com/ } \\
\text { scientific-software/prism/ }\end{array}$ & RRID:SCR_015807 & \\
\hline Software, algorithm & Java Statistical Classes library & Bertie, 2002 & & \\
\hline
\end{tabular}

\section{Animals}

Constitutive RIBEYE knockout mice (RBE ${ }^{\mathrm{KO} / K O}$ derived from Ctbp $2^{\text {tm1.2Sud }}$ by Cre-recombination) were generated by Maxeiner and colleagues (Maxeiner et al., 2016) and were back-crossed to C57BL/6 for five generations (corresponding to a C57BL/6 background contribution of $>95 \%$ ). All experiments complied with national animal care guidelines and were approved by the University of Göttingen Board for Animal Welfare and the Animal Welfare Office of the State of Lower Saxony (permit number: 14-1391). The colony was maintained by mating heterozygous mice. Whenever possible, experiments were performed in parallel on mutant mice and their wildtype littermates. However, the experimental schedule did not always permit this and we occasionally used individual mice from the same colony but without littermate controls. Moreover, for some experiments giving rise to Figure 6-figure supplement 1 and Figure 7E,F, G,H we also used C57Bl/6 wild-type mice and combined their results with those of wildtype littermate controls.

\section{Patch-clamp and confocal $\mathrm{Ca}^{2+}$-imaging}

The apical 2/3 turns of organs of Corti from P14 to P28 aged mice were freshly dissected in HEPES Hank's solution containing (in mM): $5.36 \mathrm{KCl}, 141.7 \mathrm{NaCl}, 10 \mathrm{HEPES}, 0.5 \mathrm{MgSO}_{4} .7 \mathrm{H}_{2} \mathrm{O}, 1 \mathrm{MgCl}_{2^{-}}$ $6 \mathrm{H}_{2} \mathrm{O}, 5.6 \mathrm{D}$-glucose, and $3.4 \mathrm{~L}$-glutamine ( $\left.\mathrm{pH} 7.2, \sim 300 \mathrm{mOsm}\right)$. The basolateral membranes of the $\mathrm{IHCs}$ were exposed by carefully removing the surrounding cells with a suction pipette. All experiments were conducted at room temperature $\left(20-25^{\circ} \mathrm{C}\right)$.

Perforated-patch-clamp recordings were performed as described previously (Moser and Beutner, 2000). The pipette solution contained (in $\mathrm{mM}$ ): $130 \mathrm{Cs}$-gluconate, 10 tetraethylammonium (TEA)- $\mathrm{Cl}$, 10 4-AP, 10 HEPES, $1 \mathrm{MgCl}_{2}$, as well as $300 \mu \mathrm{g} / \mathrm{ml}$ amphotericin B (pH 7.2, 280 mOsm). The extracellular solution contained the following (in $\mathrm{mM}$ ): $112 \mathrm{NaCl}, 35$ TEA-Cl, $2.8 \mathrm{KCl}, 1 \mathrm{MgCl}_{2}, 1 \mathrm{CsCl}, 10$ HEPES, $2 \mathrm{CaCl}_{2}$, and $11.1 \mathrm{D}$-glucose $(\mathrm{pH} 7.2, \sim 305 \mathrm{mOsm})$. External solution change was done by bath exchange through a perfusion system.

For live-imaging, the patch pipette solution contained (in $\mathrm{mM}$ ): $111 \mathrm{Cs}$-glutamate, $1 \mathrm{MgCl}_{2}, 1$ $\mathrm{CaCl}_{2}, 10$ EGTA, 13 TEA-Cl, 20 HEPES, 4 Mg-ATP, $0.3 \mathrm{Na-GTP}$ and 1 L-Glutathione (pH 7.3, 290 mOsm). To visualize the $\mathrm{Ca}^{2+}$-hotspots and the ribbons, the $\mathrm{Ca}^{2+}$-indicator Fluo-4FF penta- $\mathrm{K}^{+}$salt (0.8 mM, Life Technologies, Germany) and the TAMRA-conjugated CtBP2/RIBEYE-binding dimer peptide $(10 \mu \mathrm{M}$, Biosynthan, Germany) were added to the intracellular solution. The extracellular solution contained the following (in mM): $2.8 \mathrm{KCl}, 102 \mathrm{NaCl}, 10 \mathrm{HEPES}, 1 \mathrm{CsCl}_{2}, 1 \mathrm{MgCl}_{2}, 5 \mathrm{CaCl}_{2}$, $35 \mathrm{TEA}-\mathrm{Cl}$, and $11.1 \mathrm{D}-\mathrm{Glucose}$ (pH 7.2, 300 mOsm).

EPC-10 amplifiers controlled by Patchmaster or Pulse software (HEKA Elektronik, Germany) were used for the measurements. IHCs were held at $-87 \mathrm{mV}$ or $-69 \mathrm{mV}$. All voltages were corrected for liquid junction potential offline ( $17 \mathrm{mV}$ or $14 \mathrm{mV}$, depending on intra- and extracellular solutions used) and voltage-drops across the series resistance $\left(R_{\mathrm{s}}\right)$. Currents were leak corrected using a $p / 10$ protocol in exocytosis experiments. Recordings were discarded when the leak current exceeded $-55 \mathrm{pA}, \mathrm{R}_{\mathrm{s}}$ exceeded $30 \mathrm{M} \Omega$ (for perforated-patch) or $15 \mathrm{M} \Omega$ within 4 min after break-in (for ruptured-patch), or $\mathrm{Ca}^{2+}$-current rundown exceeded $25 \%$. All passive electrical properties of the patchclamp recording experiments are detailed in Supplementary file 2.

Exocytosis was studied by measuring the membrane capacitance increments $\left(\Delta C_{m}\right)$ using the Lindau-Neher technique (Lindau and Neher, 1988). Cells were stimulated by step depolarizations of 
different durations to -17 or $-14 \mathrm{mV}$, or by $100 \mathrm{~ms}$ pulses to voltages ranging from -53 to -37 $\mathrm{mV}$. A resting interval of 10-100 s between the stimuli was used. Each protocol was applied two to three times and only IHCs with reproducible exocytosis during the rounds were included. For display, traces were subjected to 1, 5 or 10 pass Binomial Smoothing using Igor Pro. Current-voltage relationships ('IVs') displayed in Figure 5A (ruptured-patch, 3-week-old mice) were obtained by applying $20 \mathrm{~ms}$ depolarizing step pulses of increasing voltage from -82 to $63 \mathrm{mV}$ in $5 \mathrm{mV}$ steps.

$\mathrm{Ca}^{2+}$-imaging was performed with a spinning disk confocal scanner (CSU22, Yokogawa, Germany) mounted on an upright microscope (Axio Examiner, Zeiss, Germany) with 63x, 1.0 NA objective (W Plan-Apochromat, Zeiss). Images were acquired by a scientific CMOS camera (Neo, Andor, Germany). $\mathrm{Ca}^{2+}$-indicator F4FF and TAMRA-conjugated peptide were excited by diodepumped solid-state lasers with $491 \mathrm{~nm}$ and $561 \mathrm{~nm}$ wavelength, respectively (Cobolt $A B$ ). The spinning disk was set to $2000 \mathrm{rpm}$ to synchronize with the $10 \mathrm{~ms}$ acquisition time of the camera.

Using a piezo positioner for the objective (Piezosystem, Germany), a scan of the entire cell was performed $4 \mathrm{~min}$ after breaking into the cell, taking sections each $0.5 \mu \mathrm{m}$ at an exposure time of 0.5 $\mathrm{s}$ in the red (TAMRA-peptide) channel from the bottom to the top of the cell. In order to study the voltage-dependence of $\mathrm{Ca}^{2+}$-indicator fluorescence increments at the synapses, the confocal scans were acquired every $0.5 \mu \mathrm{m}$ from the bottom to the top ribbon in the RBE ${ }^{\mathrm{WT}} / \mathrm{WT}$ mice. For the $\mathrm{RBE}^{\mathrm{KO} / \mathrm{KO}}$ mice, the scanning was done from the bottom of the cell to $+12 \mu \mathrm{m}$, which on average corresponds to the bottom of the nucleus. $\mathrm{Ca}^{2+}$-currents were evoked by applying a voltage ramp stimulus from -87 to $+63 \mathrm{mV}$ during $150 \mathrm{~ms}(1 \mathrm{mV} / \mathrm{ms})$ in each focal plane. Simultaneously, fluorescence measurements were made in the green channel (Fluo-4FF) with a frame rate of $100 \mathrm{~Hz}$. In order to overcome the limitations of the frame rate and increase the voltage resolution of the fluorescent signal acquired, the voltage ramp protocol was applied twice, once shifted by $5 \mathrm{~ms}$ such that for any given frame during the second ramp the voltage was shifted by $5 \mathrm{mV}$ compared to the first stimulus. Alternating planes were acquired to avoid photobleaching encountered with the consecutive plane acquisition.

\section{Immunohistochemistry, confocal and high resolution STED imaging}

Apical turns of organs of Corti from 3-week-old mice were prepared for 'whole-mount imaging' as described in (Ohn et al., 2016). In brief, the samples were fixed either in formaldehyde (4\%, $10 \mathrm{~min}$ on ice), or methanol $\left(20 \mathrm{~min}\right.$ at $\left.-20^{\circ} \mathrm{C}\right)$. Afterwards, the following primary antibodies were used: mouse anti-CtBP2 (1:200, BD Biosciences, Germany 612044), mouse anti-PSD-95 (1:200, Sigma Aldrich, Germany P246-100ul), mouse anti-bassoon SAP7f407 (1:200, Abcam, Germany, ab82958), guinea pig anti-bassoon (1:500, Synaptic Systems, Germany, 141 004), rabbit anti-RIM2 (1:100, Synaptic Systems 140 103), rabbit anti-Ca 1.3 (1:75 or 1:100, Alomone Labs, Germany, ACC 005), rabbit anti-piccolino (1:500, kind gift of JH Brandstätter; see Regus-Leidig et al., 2013), guinea pig antiparvalbumin $\alpha$ (1:1000, Synaptic Systems, 195 004), mouse anti-calbindin 28 k (1:500, Swant, Germany, 07(F)), and rabbit anti-calretinin (1:1000, Swant 1893-0114). Secondary antibodies used were Alexa Fluor 488 conjugated anti-rabbit, Alexa Fluor 488 conjugated anti-guinea-pig, Alexa Fluor 568 conjugated anti-mouse, and Alexa Fluor 647 conjugated anti-rabbit (1:200, Invitrogen, Germany, A11008, A11004, A11073, and A31573 respectively). For high resolution STED microscopy, STAR580 and STAR635p conjugated anti-rabbit and anti-mouse (1:200, Abberior, Germany, 2-0002-005-1, 2-0012-005-8, 2-0002-007-5, and 2-0012-007-2) have been used as secondary antibodies. Images were acquired using either a Leica SP5 with a 1.4 NA $63 x$ oil immersion objective or an Abberior Instruments Expert Line STED microscope, with excitation lasers at 488, 561, and $633 \mathrm{~nm}$ and STED lasers at $595 \mathrm{~nm}, 1 \mathrm{~W}$, and $775 \mathrm{~nm}, 1.2 \mathrm{~W}$, using a 1.4 NA 100x oil immersion objective, either in confocal or in 2D-STED mode. Images were adjusted for brightness and contrast using ImageJ.

\section{Systems physiology: Auditory Brainstem Responses (ABR), Distortion Product Otoacoustic Emissions (DPOAE) and extracellular recordings from SGNs}

$A B R, D P O A E$ and extracellular recordings from single SGNs were performed essentially as described before (Jing et al., 2013; Strenzke et al., 2016). ABR and DPOAE recordings were performed on 6week-old mice. For extracellular recordings from individual SGNs, 6 to 10 week-old mice were 
anesthetized by i.p. injection of urethane $(1.32 \mathrm{mg} / \mathrm{kg})$, xylazine $(5 \mathrm{mg} / \mathrm{kg})$ and buprenorphine $(0.1$ $\mathrm{mg} / \mathrm{kg}$ ), a tracheostomy was performed and the mice were then placed in a stereotactic system. After partial removal of the occipital bone and cerebellum to expose the anteroventral cochlear nucleus (AVCN), a glass microelectrode was advanced through the posterior AVCN portion to reach the auditory nerve. Acoustic stimulation was provided by an open field Avisoft ScanSpeak Ultrasonic Speaker (Avisoft Bioacoustics, Germany), and 'putative' SGNs (auditory nerve fibers formed by the central SGN axons) were identified and distinguished from cochlear nucleus neurons based on their stereotactic position (>1.1 $\mathrm{mm}$ from the surface of the cochlear nucleus), spontaneous and noiseburst induced firing, peristimulus time histogram (PSTH), regularity of firing, and first spike latency. Recordings were performed using TDT system III hardware and an ELC-03XS amplifier (NPI Electronics, Germany), offline analysis using waveform-based spike detection using custom-written MATLAB software (Source code 1).

\section{Transmission electron microscopy}

Conventional embedding

Conventional embedding of organs of Corti was essentially performed as described previously (Wong et al., 2014). In brief, here P21, 6 weeks and 8 months old mice were used. The apical turn of organs of Corti were dissected in phosphate-buffer saline (PBS) and fixed for $1 \mathrm{hr}$ on ice with $4 \%$ paraformaldehyde and $0.5 \%$ glutaraldehyde in PBS $(\mathrm{pH} 7.4)$. After an additional fixation overnight on ice with $2 \%$ glutaraldehyde in $0.1 \mathrm{M}$ sodium cacodylate buffer $(\mathrm{pH} 7.2)$, samples were washed in $0.1 \mathrm{M}$ sodium cacodylate buffer and placed in $1 \%$ osmium tetroxide $(\mathrm{v} / \mathrm{v}$ in $0.1 \mathrm{M}$ sodium cacodylate buffer) on ice for $1 \mathrm{hr}$. Next, samples were washed twice in $0.1 \mathrm{M}$ sodium cacodylate buffer (10 min each, on ice) and further in distilled water and subsequently en bloc stained with $1 \%$ uranyl acetate ( $\mathrm{v} / \mathrm{v}$ in distilled water) for $1 \mathrm{hr}$ on ice. Uranyl acetate treated samples were briefly washed three times in distilled water, dehydrated using a series of increasing ethanol concentration and finally embedded in epoxy resin (Agar $100 \mathrm{kit}$, Plano, Germany) and polymerized for $48 \mathrm{hr}$ at $70^{\circ} \mathrm{C}$. An Ultracut E microtome (Leica Microsystems, Germany) equipped with a $35^{\circ}$ diamond knife (Diatome, Switzerland) was used to obtain ultrathin sections $(70-75 \mathrm{~nm})$ of the specimen. Sections were transferred to $1 \%$ formvar-coated ( $\mathrm{w} / \mathrm{v}$ in water-free chloroform) copper slot grids (ATHENE copper slot grids, 3.05 $\mathrm{mm} \varnothing, 1 \mathrm{~mm} \times 2 \mathrm{~mm}$; Plano, Germany) and subsequently stained with uranyl acetate replacement solution (UAR-EMS) (Science Services, Germany) and Reynold's lead citrate. The specimens were investigated at $80 \mathrm{kV}$ with a JEM1011 transmission electron microscope (JEOL, Germany) and micrographs acquired at 10,000-x magnification using a Gatan Orius 1200A camera (Gatan Germany, using the Digital Micrograph software package). Serial 3D reconstructions of ultrathin sections were generated with the program Reconstruct (Fiala, 2005).

\section{High-pressure freezing/freeze-substitution (HPF/FS) and electron tomography}

High-pressure freezing, freeze-substitution followed by electron tomography were essentially performed as described previously (Vogl et al., 2015; Jung et al., 2015a). After freeze-substitution and embedding in epoxy resin (Agar 100 kit, Plano, Germany), $250 \mathrm{~nm}$ semithin sections for electron tomography were obtained on an Ultracut E ultramicrotome (Leica Microsystems, Germany) with a $35^{\circ}$ diamond knife (Diatome, Switzerland). Sections were placed on $1 \%$ formvar-coated $(\mathrm{w} / \mathrm{v}$ in water-free chloroform) copper 100 mesh grids (ATHENE, Plano, Germany, $3.05 \mathrm{~mm} \varnothing$ ) and poststained with UAR-EMS (Science Services, Germany) and Reynold's lead citrate.

For electron tomography, $10 \mathrm{~nm}$ gold particles (British Bio Cell/Plano, Germany) were applied to both sides of the stained grids. Single tilt series at $12,000-x$ magnification, mainly from -60 to $+60^{\circ}$ (if only fewer angles were possible, the tomograms were only accepted for quantification if the quality was sufficient) were acquired with an $1^{\circ}$ increment at a JEM2100 (JEOL, Germany)) transmission electron microscope at $200 \mathrm{kV}$ using the Serial-EM software (Mastronarde, 2005). The tomograms were generated using the IMOD package etomo and models were generated using $3 \mathrm{dmod}$ (Kremer et al., 1996). 


\section{Data analysis}

Live-imaging and IHC-patch-clamp data were analyzed using custom programs in Igor Pro 6.3 (Wavemetrics, Portland, OR, USA; Source Code 2). For analysis of IV-curves, the evoked $\mathrm{Ca}^{2+}$-current was averaged from 5 to $10 \mathrm{~ms}$ after the start of the depolarization. The total $\mathrm{Ca}^{2+}$-charge was estimated by taking the integral of the leak-subtracted current during the depolarization step. For most protocols, $\Delta C_{m}$ was estimated as the difference between the mean of $C_{m} 400 \mathrm{~ms}$ after and before the depolarization (the initial $60 \mathrm{~ms}$ after the end of depolarization were skipped). For paired pulse experiments, the calculation of the mean of $C_{m}$ before and after the depolarization was limited to the time remaining in the inter pulse interval after skipping (the initial $30 \mathrm{~ms}$ after the end of depolarization were skipped).

$\Delta \mathrm{F}$ images were generated by subtracting the fluorescence intensity inside the cell at the resting state $\left(F_{0}\right.$, an average of 10 frames) from the one at the depolarized state (an average of 6 frames during voltage ramp protocol). $\Delta \mathrm{F}$ was calculated as the average of a $3 \times 3$ pixel square placed in the region showing the greatest intensity increase within the fluorescence hotspot. Maximal $\Delta \mathrm{F}$ $\left(\Delta \mathrm{F}_{\max }\right)$ was the average of $5 \Delta \mathrm{F}$ values obtained between -17 and $+8 \mathrm{mV}$ during the voltage ramp

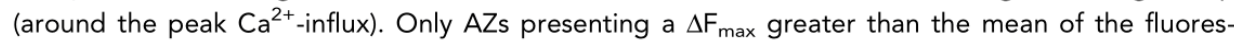
cence intensity plus two standard deviations at rest were considered for further analysis. For analysis of the voltage dependence of synaptic $\mathrm{Ca}^{2+}$-signals, raw traces were fitted to the following

$$
F(V)=F_{0}+\frac{f_{v} \cdot\left(V_{r}-V\right)}{1+e^{\frac{\left(V_{h}-V\right)}{k}}}
$$

where $f_{v}$ is the fluorescence-voltage-relationship $\Delta \mathrm{F} / \Delta \mathrm{V}$ obtained by linear fitting to the $\mathrm{FV}$-curve in the range of 3 to $23 \mathrm{mV}, V_{r}$ the reversal potential of $65.6 \mathrm{mV}$, and $V$ the command voltage, in order to obtain $V_{h}$, the voltage of half-maximal activation, and $k$, the slope factor. The spatial extent of the synaptic $\mathrm{Ca}^{2+}$-signals was estimated by fitting of a $2 \mathrm{D}$ Gaussian function to the fluorescent hotspot using a genetic fit algorithm (Sanchez del Rio and Pareschi, 2001) to obtain the full width at half maximum in the long and short axis. For each spot, the calculations were made at those confocal sections where the intensity of the spot was strongest.

Activation time constants of $\mathrm{Ca}^{2+}$-currents at differing potentials were obtained by fitting to the first $5 \mathrm{~ms}$ of the current traces the following equation:

$$
f(t)=y_{0}+A \times\left(1-e^{\left(\frac{(-x)}{\tau}\right)}\right)^{2}
$$

Confocal and STED immunofluorescence images were analyzed and z-projected with Fiji software and further analyzed using Igor Pro. For synapse counting, co-localized pre- and postsynaptic immunofluorescent spots were counted manually. The spatial extent of the line-shaped $\mathrm{Ca}^{2+}$-channel clusters was estimated by fitting a 2D Gaussian function to the individual clusters in 2D STED images to obtain the full width at half maximum in the long and short axis. The areas of the PSD were calculated by the following formula: area $=\pi \times$ (Long Axis/2) (Short Axis/2). The semi-quantitative immunofluorescence analysis of the proteinaceous $\mathrm{Ca}^{2+}$-buffers was performed by calculating the mean immunofluorescence intensity of a volume $(40(X) \times 40(Y) \times 4(Z)$ voxels or $2.8 \times 2.8 \times 2 \mu \mathrm{m})$ below the nucleus and above the synapses. This and the count and intensity of the CtBP2 immunofluorescent spots have been analyzed in Imaris 7.6 .5 with custom Matlab routines (Source Code 4).

For extracellular SGN recordings, PSTHs were calculated as average firing rates across 200 presentations of $50 \mathrm{~ms}$ or $500 \mathrm{~ms}$ tone bursts presented at $0.1 \mathrm{~s} / 0.2 \mathrm{~s}$ or $2 \mathrm{~s}$ intervals, resp. (PSTH at 10/ $5 \mathrm{~Hz}$ and $0.5 \mathrm{~Hz}$ ) at $\mathrm{C}_{\mathrm{f}}, 30 \mathrm{~dB}$ above the threshold and binned at a width of $2 \mathrm{~ms}$. Peak rate was determined as the largest bin of the PSTH in a time window 3-11 ms after stimulus onset. Adapted rate was averaged in a window spanning 35-45 ms or 405-415 ms after stimulus onset (for PSTH at $5 \mathrm{~Hz}$ and $0.5 \mathrm{~Hz}$, respectively). Rate level functions were acquired using $50 \mathrm{~ms}$ tone bursts presented at $\mathrm{C}_{\mathrm{f}}$ and at $5 \mathrm{~Hz} .25$ repetitions for each stimulus intensity ( $5 \mathrm{~dB}$ steps) were recorded. Maximal steepness was calculated as the maximal increase in spike rate between two consecutive $5 \mathrm{~dB}$ increment steps. Dynamic range was calculated by using sigmoidal fits in the rate level functions as described in and measuring the range of sound pressure between $10 \%$ and $90 \%$ of maximal firing rate. For amplitude modulation analysis, synchronization index was calculated as described by 
(Goldberg and Brown, 1969). Synchronization index estimation was only considered valid when at least 15 spikes occurred in a $3 \mathrm{~s}$ time window and the Rayleigh statistic was below 13.8.

For analysis of forward masking experiments, spike counts in a $10 \mathrm{~ms}$ interval starting from responses of both masker and probe onset were determined and presented as the ratio of probe and masker responses for at least 25 repetitions for every masker-probe interval from each unit. Exponential fitting to the plots of each individual SGN approximated the recovery kinetics.

Quantitative analysis of electron microscopy data was performed with ImageJ for conventional embedded samples and with IMOD for HPF/FS tomograms. According to the presence of ribbonoccupied and ribbonless synapses, we considered the following analysis criteria:

For ribbon-occupied synapses, membrane-proximal synaptic vesicles (MP-SVs, within a distance of $\leq 25 \mathrm{~nm}$ from the $A Z$ membrane and $\leq 80 \mathrm{~nm}$ from the presynaptic density) and ribbon-associated synaptic vesicles (RA-SVs, first layer around the ribbon with a maximum distance of $80 \mathrm{~nm}$ from the vesicle membrane to the ribbon) were counted (Figure $2 \mathrm{~J}$, random sections analysis according to Strenzke et al., 2016 and Figure 3G for tomograms according to 2D-random section analysis criteria). The tomogram analysis parameters were further modified, as used in Jung et al., 2015a. Here, the MP-SVs were defined as vesicles with $\leq 50 \mathrm{~nm}$ from the $\mathrm{AZ}$ membrane and with the shortest distance from the vesicle membrane to the presynaptic density of $\leq 100 \mathrm{~nm}$, excluding RA-SVs (Figure 3-figure supplement 1A). For random sections, SV diameters were calculated by the averaged measurements of the horizontal and vertical axis. The ribbon size was measured in height and width, taking the longest axis of the ribbon excluding the PD, and the edges of the synaptic ribbon were traced manually using ImageJ. The length of the PD was measured along the AZ membrane (Figure 2J).

For ribbonless synapses, a presynaptic density-associated synaptic vesicle (PDA-SVs) pool was defined considering all clustered vesicles $\leq 80 \mathrm{~nm}$ around the PD that did not fulfill the criteria of a MP-SV (see above, also Figure 2J for random sections). The MP-SV pool, as well as the SV diameter and PD length, were analyzed as for the ribbon-occupied synapses. For tomograms, the PDA-SV pool was defined as the SVs in the first layer $\leq 80 \mathrm{~nm}$ to the PD, excluding the MP-SVs. The MP-SV pool criteria are the same as described in the previous paragraph (Figure 3G, according to 2D-random section and Figure 3-figure supplement 1A, according to Jung et al., 2015a). For tomograms, the according pools were further distinguished into tethered and non-tethered vesicles (Figure 3G and Figure 3-figure supplement 1A). All vesicles were annotated using a spherical 'scattered object' at its maximum projection in the tomogram, encompassing the outer leaflet of the vesicles. The vesicle radii were determined automatically (Helmprobst et al., 2015) with the program 'imodinfo option - $p$ ' of the IMOD software package (Kremer et al., 1996).

\section{Release site model of RRP release and replenishment}

The coding of sound onset differs among the various SGNs in time due to different durations of the traveling wave, synaptic delays and conduction times. To obtain an average PSTH for modeling that is not smeared out due to such differences between units, the individual PSTHs were aligned beforehand by shifting their timing relative to each other. Onset detection was based on a change in spike statistics. For spontaneous activity, the 99.5 percentile of spike counts was determined. Next, the time at which response rises to twice this percentile was found. This is certainly a point within the sound response. Finally going back from this point, a drop back baseline activity, that is below the percentile was detected and used as onset time. Aligned PSTH from all units were averaged. This averaged PSTH from the forward masking data were fit with a model waveform using a genetic fit algorithm implemented in IGOR Pro (Wavemetrics, Lake Oswego, OR, USA). The purpose of the model is to give insight into the dynamics of SV cycling at the average IHC AZ. More specifically, the notion of $\mathrm{Ca}^{2+}$-nanodomain-like control of RRP exocytosis (Brandt et al., 2005; Graydon et al., 2011; Pangršič et al., 2015; Wong et al., 2014), as well as the limited MP-SVs at the AZ (see Figures 2 and 3) motivates the notion of a limited, quasi-fixed number of available vesicular release sites or slots, $\mathrm{N}_{\text {slot, }}$ (Frank et al., 2010; Wong et al., 2014) that constitute the RRP. Each of these sites can be either empty or occupied by a release ready SV (whereby all filled slots constitute the 'standing' RRP) and at each time point, a release ready SV will fuse with a certain probability described by the fusion rate constant $k_{\text {fus }}$. Its value depends on the sound pressure level in a relation we assume to be linear within the dynamic range of the synapse/fiber. While the sound pressure level rises from silence to saturation $k_{\text {fus }}$ increases from $k_{\text {fus, spont }}$ to $k_{\text {fus,stim. }}$. The refilling of empty 
sites is described by a refill rate constant $k_{\text {refill, }}$ which also depends on the sound intensity ( $k_{\text {refill, spont }}$ to $k_{\text {refill, stim). }}$.

The state of the release site was described by:

$$
\frac{d N_{\text {slot }}^{\text {fill }}(t)}{d t}=k_{\text {refill }}(t) N_{\text {slot }}-N_{\text {slot }}^{\text {filled }}(t)\left(k_{\text {fus }}(t)+k_{\text {refill }}(t)\right)
$$

Although this equation is formulated for $\mathrm{SV}$ fusion rates, a scaling factor $f$ can be used to account for the fraction of fusion events that cannot successfully trigger an action potential (AP) despite sufficient neural excitability for example because of the too small size of the elicited excitatory post synaptic current. This factor effectively operates as if the number of release sites was scaled down. The scaled equation then gives a rate R of potentially supra-threshold EPSCs as the product of the number of occupied release sites, the fusion rate constant and the scaling factor $\mathrm{f}$ :

$$
R(t)=k_{\text {fus }}(t) \cdot f \cdot N_{\text {slot }}^{\text {filled }}(t)
$$

The stationary solutions of Equation 3 together with Equation 4 determine steady state occupancy and steady state event rates:

$$
\begin{aligned}
& N_{\text {solt }}^{\text {filled }}||_{\text {strady state }}^{\text {condition }}=\frac{K_{\text {refill }}^{\text {condition }}}{K_{\text {fusion }}^{\text {condion }}+K_{\text {refill }}^{\text {condition }}} \cdot N_{\text {solt }} \\
& \left.R\right|_{\mid \text {strady state }} ^{\text {condion }}=\frac{K_{\text {fusion }}^{\text {condition }} \cdot f \cdot K_{\text {refill }}^{\text {condition }}}{K_{\text {fusion }}^{\text {condition }}+K_{\text {refill }}^{\text {condition }}} \cdot N_{\text {solt }}
\end{aligned}
$$

In this equation, 'condition' is either silence or saturating sound pressure level.

In order to connect the postsynaptic event rate of potentially supra-threshold EPSCs to the actual AP rate, refractoriness is considered as a combination of an absolute refractory period $t_{a b s}$, during which the probability of an EPSC to trigger an AP is zero, with a relative refractory period during which this trigger probability returns to one with an exponential time course characterized by $\tau_{\text {rel }}$ (Berry and Meister, 1998). This description of refractoriness can be applied to the 'driving' EPSC rate $\mathrm{R}$ by means of a delayed differential equation. The equation is motivated by the concept of three possible states of the SGN: 'absolute refractory', 'relative refractory' or 'available' (fully excitable). At any point, the probability that the SGN turns from 'available' to 'refractory' is proportional to the rate $R(t)$. The return back to 'available' happens 'delayed' by $t_{a b s}$ and with a probability that is proportional to $1 / \tau_{\text {rel }}$.

$$
\begin{gathered}
\frac{d f_{\text {waia }}(t)}{d t}=\frac{f_{\text {relef }}(t)}{\tau_{r e l}}-f_{\text {avail }}(t) \cdot f \cdot R(t) \\
\frac{d f_{\text {relerf }}(t)}{d t}=f_{\text {avail }}\left(t-t_{\text {abs }}\right) \cdot f \cdot R\left(t-t_{\text {abs }}\right)-\frac{f_{\text {relref }}(t)}{\tau_{\text {rel }}}
\end{gathered}
$$

Together with Equation 6 the stationary solution of this description of refractoriness connects the observable steady state rates during silence and stimulation to the rate constants $k_{\text {refill }}$ und $k_{\text {fusion: }}$ :

$$
A P-\left.R a t e\right|_{\text {strady state }} ^{\text {condition }}=\frac{K_{\text {fision }}^{\text {condition }} \cdot f \cdot K_{\text {refili }}^{\text {condion }}}{K_{\text {funsion }}^{\text {condition }}+K_{\text {refill }}^{\text {condion }}} \cdot N_{\text {solt }} \cdot \frac{1}{1+R||_{\mid \text {strady state }}^{\text {condition }}\left(t_{\text {abs }}+\tau_{\text {rel }}\right)}
$$

To go beyond the description of steady state event rates and to use the model for a parameterized description of the actual time course of experimentally observed PSTHs acquired during forward masking (Figure 11E), it is necessary to define the relation between the applied stimulus and the fusion and refill rate constants. For the experimental data presented here, the stimulus level was increased from silence to $30 \mathrm{~dB}$ above fiber threshold within a $4 \mathrm{~ms}$ ramp having a quarter of a $\sin ^{2}$ shape. It was assumed that $k_{\text {fusion }}$ and $k_{\text {refill }}$ follow the stimulus increase simultaneously. The ordinary differential and delayed differential equations above were combined into a fit function. PSTHs (one masker followed by one probe) were averaged per genotype for each masker probe interval $(4,16$, 64 and $256 \mathrm{~ms}$ ) and were fitted in parallel with one parameter set. During experiments, trials were acquired in immediate succession without gaps. Therefore, the model implements cyclic boundary conditions for the occupancy of the slots. This model only captures the short term processes, 
assuming that a set of experiments, for example forward masking trials quickly lead to a steady state. Slow adapting processes were not explicitly modeled. The observed drop of the apparent number of available slots in the forward masking experiments was described here as a change in the number of slots from $\mathrm{N}_{\text {slots }}$ to a reduced capacity $\mathrm{N}_{\text {slots }}$ and for a given spiral ganglion neuron that was tested with tone bursts and forward masking, the ratio $\mathrm{N}_{\text {slots }} / \mathrm{N}^{\prime}$ slots could be estimated from the change in rates (see Results).

\section{Statistical analysis}

The data were analyzed using Matlab (Mathworks), Excel, lgor Pro 6 (Wavemetrics), Origin 9.0 (Microcal Software), and GraphPad Prism (GraphPad Software). Averages were expressed as mean \pm standard error of the mean (S.E.M.). For every dataset, the standard deviation (S.D.), number of replicates $(n)$ and animals $(N)$ were indicated. For Figure $7, n_{\min }$ corresponds to the minimum number of cells included in the analysis of each depolarization potential given that the number of cells for each potential differs. In order to compare two samples, data sets were tested for normal distribution (Jarque-Bera test, D'Agostino and Pearson omnibus normality test or the Shapiro-Wilk test) and equality of variances (F-test), followed by two-tailed unpaired Student's t-test, or, when data were not normally distributed and/or variance was unequal between samples, the unpaired two-tailed Mann-Whitney-Wilcoxon test was used. Cumulative distributions in Figure 9A were statistically compared using the Kolmogorov-Smirnov test. The ROUT method $(\mathrm{Q}=0.1 \%)$ from GraphPad Prism was used to identify definitive outliers for Figure $7 \mathrm{H}$.

For multiple comparisons, statistical significance was calculated by using one-way ANOVA test (two-way ANOVA in the case of ABR thresholds) followed by Tukey's test for normally distributed data or Kruskal-Wallis (K-W) test followed by non-parametric multiple comparisons test (NPMC) for non-normally distributed data.

For SV diameter quantifications in random sections, a custom-written routine using Java Statistical Classes library (JSC) (Bertie, 2002) was utilized for statistical analysis (Source code 5). Due to the tied ranks of SV diameter measurements obtained for random sections, their S.E.M. was used as a tolerance value for the usage of Kruskal-Wallis test as suggested by Bertie et al. in JSC library (Bertie, 2002), where two values were treated as equal if their difference was $\leq$ S.E.M.. The non-significant difference between samples is reported as n.s., significant differences are reported as ${ }^{*} p<0.05$, ${ }^{\star \star} p<0.01,{ }^{\star \star \star} p<0.001,{ }^{\star \star \star *} p<0.0001$.

\section{Acknowledgements}

We thank N Dietrich, T Goldak, S Gerke, C Senger-Freitag and I Herfort for expert technical assistance, Gerhard Hoch for software and analysis development, and Frank Schmitz for providing $\mathrm{RBE}^{\mathrm{KO} / \mathrm{WT}}$ mice.

\section{Additional information}

\begin{tabular}{lll}
$\begin{array}{l}\text { Funding } \\
\text { Funder }\end{array}$ & Grant reference number & Author \\
\hline $\begin{array}{l}\text { Deutsche Forschungsge- } \\
\text { meinschaft }\end{array}$ & $\begin{array}{l}\text { Collaborative Research } \\
\text { Center 889, projects A2, A6, } \\
\text { and A7 }\end{array}$ & $\begin{array}{l}\text { Carolin Wichmann } \\
\text { Tobias Moser }\end{array}$ \\
\hline $\begin{array}{l}\text { Deutsche Forschungsge- } \\
\text { meinschaft }\end{array}$ & $\begin{array}{l}\text { Tobias Moser Leibniz } \\
\text { program MO 896/51 }\end{array}$ & Tobias Moser \\
\hline Max-Planck-Gesellschaft & Max-Planck-Fellowship & Tobias Moser \\
\hline Niedersächsisches Vorab & & Tobias Moser
\end{tabular}

The funders had no role in study design, data collection and interpretation, or the decision to submit the work for publication. 
Author contributions

Philippe Jean, Data curation, Formal analysis, Investigation, Visualization, Writing-original draft, Writing-review and editing, PJ performed immunohistochemistry and confocal immunofluorescence microscopy, patch-clamp, Ca2+ imaging, computational modeling and prepared the manuscript; David Lopez de la Morena, Data curation, Formal analysis, Investigation, Visualization, Writing-original draft, Writing-review and editing, DL performed in vivo extracellular recordings from single SGNs and AVCN neurons, and contributed to the manuscript; Susann Michanski, Data curation, Formal analysis, Investigation, Visualization, Writing-original draft, Writing-review and editing, SM performed conventional embedding and electron microscopy of random and serial sections, contributed to high-pressure freezing and to the manuscript; Lina María Jaime Tobón, Data curation, Formal analysis, Investigation, Visualization, Writing-original draft, Writing-review and editing, LMJT performed patch-clamp capacitance measurements and contributed to the manuscript; Rituparna Chakrabarti, Data curation, Formal analysis, Investigation, Visualization, Writing-original draft, Writing-review and editing, RC performed high-pressure freezing, freeze-substitution and electron tomography, and contributed to the manuscript; Maria Magdalena Picher, Data curation, Formal analysis, Investigation, Visualization, Writing-original draft, MMP performed patch-clamp capacitance measurements and contributed to the manuscript; Jakob Neef, Data curation, Software, Formal analysis, Supervision, Investigation, Visualization, Methodology, Writing-original draft, JN performed immunohistochemistry, confocal and STED immunofluorescence microscopy, co-supervised PJ and LMJT, and contributed to the manuscript; SangYong Jung, Data curation, Formal analysis, Investigation, SYJ performed immunohistochemistry and confocal immunofluorescence microscopy; Mehmet Gültas, Software, Formal analysis, Methodology, MG contributed a statistica analysis of electron microscopy data; Stephan Maxeiner, Resources, Writing-original draft, SM contributed mutant mice and genetic expertise; Andreas Neef, Conceptualization, Software, Formal analysis, Supervision, Methodology, Writing-original draft, AN designed the study, performed and supervised computational modeling, and contributed to the manuscript; Carolin Wichmann, Conceptualization, Supervision, Visualization, Writing-original draft, Writing-review and editing, CW designed the study, supervised electron microscopy and tomography, and contributed to the manuscript; Nicola Strenzke, Conceptualization, Software, Supervision, Methodology, NS designed the study and supervised in vivo extracellular recordings from single SGNs; Chad Grabner, Conceptualization, Writing-original draft, CG designed the study and contributed to the manuscript; Tobias Moser, Conceptualization, Resources, Supervision, Funding acquisition, Validation, Visualization, Methodology, Writing-original draft, Project administration, Writing-review and editing, TM designed the study, prepared the manuscript and co-supervised PJ, DL and LMJT

Author ORCIDs

Philippe Jean (iD http://orcid.org/0000-0001-5325-1370

David Lopez de la Morena (iD) http://orcid.org/0000-0003-0835-2732

Susann Michanski (id https://orcid.org/0000-0001-5893-1981

Lina María Jaime Tobón (iD http://orcid.org/0000-0002-6752-7750

Maria Magdalena Picher (iD) https://orcid.org/0000-0003-0722-3883

Jakob Neef (D) http://orcid.org/0000-0002-4757-9385

Andreas Neef (iD) https://orcid.org/0000-0003-4445-7478

Carolin Wichmann (iD http://orcid.org/0000-0001-8868-8716

Tobias Moser (iD http://orcid.org/0000-0001-7145-0533

Ethics

Animal experimentation: All experiments complied with national animal care guidelines and were approved by the University of Göttingen Board for Animal Welfare and the Animal Welfare Office of the State of Lower Saxony (permit number: 14-1391).

Decision letter and Author response

Decision letter https://doi.org/10.7554/eLife.29275.031

Author response https://doi.org/10.7554/eLife.29275.032 


\section{Additional files}

Supplementary files

- Source code 1. Matlab scripts for the waveform-based spike detection.

DOI: https://doi.org/10.7554/eLife.29275.022

- Source code 2. Live calcium imaging analysis.

DOI: https://doi.org/10.7554/eLife.29275.023

- Source Code 3. Routines for the analysis of whole-cell capacitance measurements

DOI: https://doi.org/10.7554/eLife.29275.024

- Source Code 4. Matlab scripts for the count andintensity of immunofluorescent spots.

DOI: https://doi.org/10.7554/eLife.29275.025

- Source code 5. Java routine for the SV diameter quantifications in random sections

DOI: https://doi.org/10.7554/eLife.29275.026

- Supplementary file 1. Modified tomogram analysis. The table depicts the modified tomogram analysis performed according to Jung et al., 2015a displaying the mean \pm (S.E.M.), (S.D.), p-values, sample size and statistical tests used to compare $\mathrm{RBE}^{\mathrm{WT} / \mathrm{WT}}$ and $\mathrm{RBE}^{\mathrm{KO} / \mathrm{KO}}$. Refer to Figure 3-figure supplement 1 for the graphs.

DOI: https://doi.org/10.7554/eLife.29275.027

- Supplementary file 2. Passive electrical properties of patch-clamp recording experiments.

The table shows the mean \pm (S.E.M.) of the passive electrical properties across all ruptured and perforated patch-clamp recording experiments in $\mathrm{RBE}^{\mathrm{WT} / \mathrm{WT}}$ and $\mathrm{RBE}^{\mathrm{KO} / \mathrm{KO}}$ conditions.

DOI: https://doi.org/10.7554/eLife.29275.028

- Transparent reporting form

DOI: https://doi.org/10.7554/eLife.29275.029

\section{References}

Bartoletti TM, Jackman SL, Babai N, Mercer AJ, Kramer RH, Thoreson WB. 2011. Release from the cone ribbon synapse under bright light conditions can be controlled by the opening of only a few $\mathrm{Ca}(2+)$ channels. Journal of Neurophysiology 106:2922-2935. DOI: https://doi.org/10.1152/jn.00634.2011, PMID: 21880934

Berry MJ, Meister M., 1998. Refractoriness and neural precision. Journal of Neuroscience 18:2200-2211. PMID: 9482804

Bertie A. 2002. Java applications for teaching statistics. MSOR Connections 2:78-81. DOI: https://doi.org/10. $11120 /$ msor. 2002.02030078

Brandt A, Khimich D, Moser T. 2005. Few CaV1.3 channels regulate the exocytosis of a synaptic vesicle at the hair cell ribbon synapse. Journal of Neuroscience 25:11577-11585. DOI: https://doi.org/10.1523/JNEUROSCl. 3411-05.2005, PMID: 16354915

Brandt A, Striessnig J, Moser T. 2003. CaV1.3 channels are essential for development and presynaptic activity of cochlear inner hair cells. Journal of Neuroscience 23:10832-10840. PMID: 14645476

Bunt AH. 1971. Enzymatic digestion of synaptic ribbons in amphibian retinal photoreceptors. Brain Research 25 : 571-577. DOI: https://doi.org/10.1016/0006-8993(71)90461-6, PMID: 5544325

Buran BN, Strenzke N, Neef A, Gundelfinger ED, Moser T, Liberman MC. 2010. Onset coding is degraded in auditory nerve fibers from mutant mice lacking synaptic ribbons. Journal of Neuroscience 30:7587-7597. DOI: https://doi.org/10.1523/JNEUROSCI.0389-10.2010, PMID: 20519533

Cole AA, Chen X, Reese TS. 2016. A Network of three types of filaments organizes synaptic vesicles for storage, mobilization, and docking. Journal of Neuroscience 36:3222-3230. DOI: https://doi.org/10.1523/JNEUROSCI. 2939-15.2016, PMID: 26985032

Cui G, Meyer AC, Calin-Jageman I, Neef J, Haeseleer F, Moser T, Lee A. 2007. Ca2+-binding proteins tune Ca2 +-feedback to Cav1.3 channels in mouse auditory hair cells. The Journal of physiology 585:791-803. DOl: https://doi.org/10.1113/jphysiol.2007.142307, PMID: 17947313

Davydova D, Marini C, King C, Klueva J, Bischof F, Romorini S, Montenegro-Venegas C, Heine M, Schneider R, Schröder MS, Altrock WD, Henneberger C, Rusakov DA, Gundelfinger ED, Fejtova A. 2014. Bassoon specifically controls presynaptic P/Q-type $\mathrm{Ca}(2+)$ channels via RIM-binding protein. Neuron 82:181-194. DOI: https://doi.org/10.1016/j.neuron.2014.02.012, PMID: 24698275

Dick O, Hack I, Altrock WD, Garner CC, Gundelfinger ED, Brandstätter JH. 2001. Localization of the presynaptic cytomatrix protein piccolo at ribbon and conventional synapses in the rat retina: comparison with Bassoon. Journal of Comparative Neurology 439:224-234. DOI: https://doi.org/10.1002/cne.1344, PMID: 11596050 
Dick O, tom Dieck S, Altrock WD, Ammermüller J, Weiler R, Garner CC, Gundelfinger ED, Brandstätter JH. 2003 The presynaptic active zone protein bassoon is essential for photoreceptor ribbon synapse formation in the retina. Neuron 37:775-786. DOI: https://doi.org/10.1016/S0896-6273(03)00086-2, PMID: 12628168

Dou H, Vazquez AE, Namkung Y, Chu H, Cardell EL, Nie L, Parson S, Shin HS, Yamoah EN. 2004. Null mutation of alpha1D Ca2+ channel gene results in deafness but no vestibular defect in mice. Journal of the Association for Research in Otolaryngology : JARO 5:215-226. DOI: https://doi.org/10.1007/s10162-003-4020-3, PMID: 15357422

Fernández-Busnadiego R, Asano S, Oprisoreanu AM, Sakata E, Doengi M, Kochovski Z, Zürner M, Stein V, Schoch S, Baumeister W, Lucić V. 2013. Cryo-electron tomography reveals a critical role of RIM1 $\alpha$ in synaptic vesicle tethering. The Journal of Cell Biology 201:725-740. DOI: https://doi.org/10.1083/jcb.201206063, PMID: 23712261

Fiala JC. 2005. Reconstruct: a free editor for serial section microscopy. Journal of Microscopy 218:52-61. DOI: https://doi.org/10.1111/j.1365-2818.2005.01466.x, PMID: 15817063

Frank T, Khimich D, Neef A, Moser T. 2009. Mechanisms contributing to synaptic Ca2+ signals and their heterogeneity in hair cells. PNAS 106:4483-4488. DOI: https://doi.org/10.1073/pnas.0813213106, PMID: 1 9246382

Frank T, Rutherford MA, Strenzke N, Neef A, Pangršič T, Khimich D, Fejtova A, Fetjova A, Gundelfinger ED, Liberman MC, Harke B, Bryan KE, Lee A, Egner A, Riedel D, Moser T. 2010. Bassoon and the synaptic ribbon organize $\mathrm{Ca}^{2}+$ channels and vesicles to add release sites and promote refilling. Neuron 68:724-738. DOl: https://doi.org/10.1016/j.neuron.2010.10.027, PMID: 21092861

Goldberg JM, Brown PB. 1969. Response of binaural neurons of dog superior olivary complex to dichotic tonal stimuli: some physiological mechanisms of sound localization. Journal of Neurophysiology 32:613-636. DOI: https://doi.org/10.1152/jn.1969.32.4.613, PMID: 5810617

Grabner CP, Zenisek D. 2013. Amperometric resolution of a prespike stammer and evoked phases of fast release from retinal bipolar cells. Journal of Neuroscience 33:8144-8158. DOI: https://doi.org/10.1523/JNEUROSCl. 5062-12.2013, PMID: 23658155

Graydon CW, Cho S, Li GL, Kachar B, von Gersdorff H, Gersdorff Hvon. 2011. Sharp Ca ${ }^{2+}$ nanodomains beneath the ribbon promote highly synchronous multivesicular release at hair cell synapses. Journal of Neuroscience 31 16637-16650. DOI: https://doi.org/10.1523/JNEUROSCI.1866-11.2011, PMID: 22090491

Graydon CW, Zhang J, Oesch NW, Sousa AA, Leapman RD, Diamond JS. 2014. Passive diffusion as a mechanism underlying ribbon synapse vesicle release and resupply. Journal of Neuroscience 34:8948-8962. DOI: https:// doi.org/10.1523/JNEUROSCI.1022-14.2014, PMID: 24990916

Hallermann S, Fejtova A, Schmidt H, Weyhersmüller A, Silver RA, Gundelfinger ED, Eilers J. 2010. Bassoon speeds vesicle reloading at a central excitatory synapse. Neuron 68:710-723. DOI: https://doi.org/10.1016/j. neuron.2010.10.026, PMID: 21092860

Harris DM, Dallos P. 1979. Forward masking of auditory nerve fiber responses. Journal of Neurophysiology 42 : 1083-1107. DOI: https://doi.org/10.1152/jn.1979.42.4.1083, PMID: 479921

Heil P, Neubauer H, Irvine DR, Brown M. 2007. Spontaneous activity of auditory-nerve fibers: insights into stochastic processes at ribbon synapses. Journal of Neuroscience 27:8457-8474. DOI: https://doi.org/10.1523/ JNEUROSCI.1512-07.2007, PMID: 17670993

Helmprobst F, Frank M, Stigloher C. 2015. Presynaptic architecture of the larval zebrafish neuromuscular junction. Journal of Comparative Neurology 523:1984-1997. DOI: https://doi.org/10.1002/cne.23775, PMID: 25766140

Hibino H, Pironkova R, Onwumere O, Vologodskaia M, Hudspeth AJ, Lesage F. 2002. RIM binding proteins (RBPs) couple Rab3-interacting molecules (RIMs) to voltage-gated $\mathrm{Ca}(2+)$ channels. Neuron 34:411-423. DOI: https://doi.org/10.1016/S0896-6273(02)00667-0, PMID: 11988172

Hildebrand JD, Soriano P. 2002. Overlapping and unique roles for C-terminal binding protein 1 (CtBP1) and CtBP2 during mouse development. Molecular and Cellular Biology 22:5296-5307. DOI: https://doi.org/10. 1128/MCB.22.15.5296-5307.2002, PMID: 12101226

Huang LC, Barclay M, Lee K, Peter S, Housley GD, Thorne PR, Montgomery JM. 2012. Synaptic profiles during neurite extension, refinement and retraction in the developing cochlea. Neural Development 7:38-17. DOI: https://doi.org/10.1186/1749-8104-7-38, PMID: 23217150

Hull C, Studholme K, Yazulla S, von Gersdorff H. 2006. Diurnal changes in exocytosis and the number of synaptic ribbons at active zones of an ON-type bipolar cell terminal. Journal of Neurophysiology 96:2025-2033. DOI: https://doi.org/10.1152/jn.00364.2006, PMID: 16738212

Jackman SL, Choi SY, Thoreson WB, Rabl K, Bartoletti TM, Kramer RH. 2009. Role of the synaptic ribbon in transmitting the cone light response. Nature Neuroscience 12:303-310. DOI: https://doi.org/10.1038/nn.2267, PMID: 19219039

Jarsky T, Tian M, Singer JH. 2010. Nanodomain control of exocytosis is responsible for the signaling capability of a retinal ribbon synapse. Journal of Neuroscience 30:11885-11895. DOI: https://doi.org/10.1523/JNEUROSCl. 1415-10.2010, PMID: 20826653

Jing Z, Rutherford MA, Takago H, Frank T, Fejtova A, Khimich D, Moser T, Strenzke N. 2013. Disruption of the presynaptic cytomatrix protein bassoon degrades ribbon anchorage, multiquantal release, and sound encoding at the hair cell afferent synapse. Journal of Neuroscience 33:4456-4467. DOI: https://doi.org/10.1523/ JNEUROSCI.3491-12.2013, PMID: 23467361 
Johnson SL, Olt J, Cho S, von Gersdorff H, Marcotti W. 2017. The Coupling between Ca2+ channels and the exocytotic $\mathrm{Ca} 2+$ sensor at hair cell ribbon synapses varies tonotopically along the mature cochlea. The Journal of Neuroscience 37:2471-2484. DOI: https://doi.org/10.1523/JNEUROSCI.2867-16.2017, PMID: 28154149

Joris PX, Carney LH, Smith PH, Yin TC. 1994. Enhancement of neural synchronization in the anteroventral cochlear nucleus. I. Responses to tones at the characteristic frequency. Journal of Neurophysiology 71:10221036. DOI: https://doi.org/10.1152/jn.1994.71.3.1022, PMID: 8201399

Jung S, Maritzen T, Wichmann C, Jing Z, Neef A, Revelo NH, Al-Moyed H, Meese S, Wojcik SM, Panou I, Bulut H Schu P, Ficner R, Reisinger E, Rizzoli SO, Neef J, Strenzke N, Haucke V, Moser T. 2015b. Disruption of adaptor protein $2 \mu$ (AP-2 $\mu)$ in cochlear hair cells impairs vesicle reloading of synaptic release sites and hearing. The EMBO Journal 34:2686-2702. DOI: https://doi.org/10.15252/embj.201591885, PMID: 26446278

Jung S, Oshima-Takago T, Chakrabarti R, Wong AB, Jing Z, Yamanbaeva G, Picher MM, Wojcik SM, Göttfert F, Predoehl F, Michel K, Hell SW, Schoch S, Strenzke N, Wichmann C, Moser T. 2015a. Rab3-interacting molecules $2 \alpha$ and $2 \beta$ promote the abundance of voltage-gated $\mathrm{CaV} 1.3 \mathrm{Ca} 2+$ channels at hair cell active zones. PNAS 112:E3141-E3149. DOI: https://doi.org/10.1073/pnas.1417207112,PMID: 26034270

Khimich D, Nouvian R, Pujol R, Tom Dieck S, Egner A, Gundelfinger ED, Moser T. 2005. Hair cell synaptic ribbons are essential for synchronous auditory signalling. Nature 434:889-894. DOI: https://doi.org/10.1038/ nature03418, PMID: 15829963

Kremer JR, Mastronarde DN, McIntosh JR. 1996. Computer visualization of three-dimensional image data using IMOD. Journal of Structural Biology 116:71-76. DOI: https://doi.org/10.1006/jsbi.1996.0013, PMID: 8742726

Krinner S, Butola T, Jung S, Wichmann C, Moser T. 2017. RIM-binding protein 2 promotes a large number of $\mathrm{CaV} 1.3 \mathrm{Ca} 2+-$ channels and contributes to fast synaptic vesicle replenishment at hair cell active zones. Frontiers in Cellular Neuroscience 11:334. DOI: https://doi.org/10.3389/fncel.2017.00334, PMID: 29163046

Köppl C. 1997. Phase locking to high frequencies in the auditory nerve and cochlear nucleus magnocellularis of the barn owl, Tyto alba. Journal of Neuroscience 17:3312. PMID: 9096164

Lagnado L, Schmitz F. 2015. Ribbon synapses and visual processing in the retina. Annual Review of Vision Science 1:235-262. DOI: https://doi.org/10.1146/annurev-vision-082114-035709, PMID: 28532378

Lee A, Wong ST, Gallagher D, Li B, Storm DR, Scheuer T, Catterall WA. 1999. Ca2+/calmodulin binds to and modulates P/Q-type calcium channels. Nature 399:155-159. DOI: https://doi.org/10.1038/20194, PMID: 10335 845

Lenzi D, Crum J, Ellisman MH, Roberts WM. 2002. Depolarization redistributes synaptic membrane and creates a gradient of vesicles on the synaptic body at a ribbon synapse. Neuron 36:649-659. DOI: https://doi.org/10. 1016/S0896-6273(02)01025-5, PMID: 12441054

Li GL, Cho S, von Gersdorff H. 2014. Phase-locking precision is enhanced by multiquantal release at an auditory hair cell ribbon synapse. Neuron 83:1404-1417. DOI: https://doi.org/10.1016/j. neuron.2014.08.027, PMID: 251 99707

Liberman MC. 1978. Auditory-nerve response from cats raised in a low-noise chamber. The Journal of the Acoustical Society of America 63:442-455. DOI: https://doi.org/10.1121/1.381736, PMID: 670542

Limbach C, Laue MM, Wang X, Hu B, Thiede N, Hultqvist G, Kilimann MW. 2011. Molecular in situ topology of Aczonin/Piccolo and associated proteins at the mammalian neurotransmitter release site. PNAS 108:E392E401. DOI: https://doi.org/10.1073/pnas.1101707108, PMID: 21712437

Lindau M, Neher E. 1988. Patch-clamp techniques for time-resolved capacitance measurements in single cells. Pflugers Archiv European Journal of Physiology 411:137-146. DOI: https://doi.org/10.1007/BF00582306, PMID: 3357753

Lv C, Stewart WJ, Akanyeti O, Frederick C, Zhu J, Santos-Sacchi J, Sheets L, Liao JC, Zenisek D. 2016. Synaptic ribbons require ribeye for electron density, proper synaptic localization, and recruitment of calcium channels. Cell Reports 15:2784-2795. DOI: https://doi.org/10.1016/j.celrep.2016.05.045, PMID: 27292637

Magupalli VG, Schwarz K, Alpadi K, Natarajan S, Seigel GM, Schmitz F. 2008. Multiple RIBEYE-RIBEYE interactions create a dynamic scaffold for the formation of synaptic ribbons. Journal of Neuroscience 28:79547967. DOI: https://doi.org/10.1523/JNEUROSCI.1964-08.2008, PMID: 18685021

Mastronarde DN. 2005. Automated electron microscope tomography using robust prediction of specimen movements. Journal of Structural Biology 152:36-51. DOI: https://doi.org/10.1016/j.jsb.2005.07.007, PMID: 16182563

Maxeiner S, Luo F, Tan A, Schmitz F, Südhof TC. 2016. How to make a synaptic ribbon: RIBEYE deletion abolishes ribbons in retinal synapses and disrupts neurotransmitter release. The EMBO Journal 35:1098-1114. DOI: https://doi.org/10.15252/embj.201592701, PMID: 26929012

Mehta B, Snellman J, Chen S, Li W, Zenisek D. 2013. Synaptic ribbons influence the size and frequency of miniature-like evoked postsynaptic currents. Neuron 77:516-527. DOI: https://doi.org/10.1016/j.neuron.2012. 11.024, PMID: 23395377

Mendoza Schulz A, Jing Z, Sánchez Caro JM, Wetzel F, Dresbach T, Strenzke N, Wichmann C, Moser T. 2014. Bassoon-disruption slows vesicle replenishment and induces homeostatic plasticity at a CNS synapse. The EMBO Journal 33:n/a-527. DOI: https://doi.org/10.1002/embj.201385887, PMID: 24442636

Moser T, Beutner D. 2000. Kinetics of exocytosis and endocytosis at the cochlear inner hair cell afferent synapse of the mouse. PNAS 97:883-888. DOI: https://doi.org/10.1073/pnas.97.2.883, PMID: 10639174

Moser T, Vogl C. 2016. New insights into cochlear sound encoding. F1000Research 5:2081. DOI: https://doi.org/ 10.12688/f1000research.8924.1 
Neef J, Urban NT, Ohn T-L, Frank T, Jean P, Hell SW, Willig KI, Moser T. 2018. Quantitative optical nanophysiology of $\mathrm{Ca} 2+$ signaling at inner hair cell active zones. Nature Communications 9. DOI: https://doi. org/10.1038/s41467-017-02612-y

Oesch NW, Diamond JS. 2011. Ribbon synapses compute temporal contrast and encode luminance in retinal rod bipolar cells. Nature Neuroscience 14:1555-1561. DOI: https://doi.org/10.1038/nn.2945, PMID: 22019730

Ohn TL, Rutherford MA, Jing Z, Jung S, Duque-Afonso CJ, Hoch G, Picher MM, Scharinger A, Strenzke N, Moser T. 2016. Hair cells use active zones with different voltage dependence of $\mathrm{Ca} 2+$ influx to decompose sounds into complementary neural codes. PNAS 113:E4716-E4725. DOI: https://doi.org/10.1073/pnas.1605737113, PMID: 27462107

Pangršič T, Gabrielaitis M, Michanski S, Schwaller B, Wolf F, Strenzke N, Moser T. 2015. EF-hand protein Ca2+ buffers regulate $\mathrm{Ca} 2+$ influx and exocytosis in sensory hair cells. PNAS 112:E1028-E1037. DOI: https://doi.org/ 10.1073/pnas.1416424112, PMID: 25691754

Pangršič T, Reisinger E, Moser T. 2012. Otoferlin: a multi-C2 domain protein essential for hearing. Trends in Neurosciences 35:671-680. DOI: https://doi.org/10.1016/j.tins.2012.08.002, PMID: 22959777

Peterson BZ, DeMaria CD, Adelman JP, Yue DT. 1999. Calmodulin is the Ca2+ sensor for Ca2+-dependent inactivation of L-type calcium channels. Neuron 22:549-558. DOI: https://doi.org/10.1016/S0896-6273(00) 80709-6, PMID: 10197534

Picher MM, Gehrt A, Meese S, Ivanovic A, Predoehl F, Jung S, Schrauwen I, Dragonetti AG, Colombo R, Van Camp G, Strenzke N, Moser T, Camp GV. 2017. Ca2+-binding protein 2 inhibits Ca2+-channel inactivation in mouse inner hair cells. PNAS 114:E1717-E1726. DOI: https://doi.org/10.1073/pnas.1617533114, PMID: 281837 97

Platzer J, Engel J, Schrott-Fischer A, Stephan K, Bova S, Chen H, Zheng H, Striessnig J. 2000. Congenital deafness and sinoatrial node dysfunction in mice lacking class $D$ L-type Ca2+ channels. Cell 102:89-97. DOI: https://doi.org/10.1016/S0092-8674(00)00013-1, PMID: 10929716

Regus-Leidig H, Ott C, Löhner M, Atorf J, Fuchs M, Sedmak T, Kremers J, Fejtová A, Gundelfinger ED, Brandstätter JH. 2013. Identification and immunocytochemical characterization of Piccolino, a novel Piccolo splice variant selectively expressed at sensory ribbon synapses of the eye and ear. PLoS One 8:e70373. DOI: https://doi.org/10.1371/journal.pone.0070373, PMID: 23936420

Robertson D, Paki B. 2002. Role of L-type Ca2+ channels in transmitter release from mammalian inner hair cells. II. Single-neuron activity. Journal of Neurophysiology 87:2734-2740. DOI: https://doi.org/10.1152/jn.2002.87.6. 2734, PMID: 12037175

Russell IJ, Sellick PM. 1983. Low-frequency characteristics of intracellularly recorded receptor potentials in guinea-pig cochlear hair cells. The Journal of Physiology 338:179-206. DOI: https://doi.org/10.1113/jphysiol. 1983.sp014668, PMID: 6875955

Rutherford MA, Chapochnikov NM, Moser T. 2012. Spike encoding of neurotransmitter release timing by spiral ganglion neurons of the cochlea. Journal of Neuroscience 32:4773-4789. DOI: https://doi.org/10.1523/ JNEUROSCI.4511-11.2012, PMID: 22492033

Rutherford MA, Moser T. 2016. The Ribbon Synapse Between Type I Spiral Ganglion Neurons and Inner Hair Cells. In: Dabdoub A, Fritzsch B, Popper A. N, Fay R. R (Eds). The Primary Auditory Neurons of the Mammalian Cochlea. New York: Springer. p. 117-156. DOl: https://doi.org/10.1007/978-1-4939-3031-9_5

Safieddine S, El-Amraoui A, Petit C. 2012. The auditory hair cell ribbon synapse: from assembly to function. Annual Review of Neuroscience 35:509-528. DOI: https://doi.org/10.1146/annurev-neuro-061010-113705, PMID: 22715884

Sanchez del Rio M, Pareschi G. 2001. Global optimization and reflectivity data fitting for x-ray multilayer mirrors by means of genetic algorithms. Advances in X-Ray Optics 88-96. DOI: https://doi.org/10.1117/12.411624

Schmitz F, Königstorfer A, Südhof TC. 2000. RIBEYE, a component of synaptic ribbons: a protein's journey through evolution provides insight into synaptic ribbon function. Neuron 28:857-872. DOI: https://doi.org/10 1016/S0896-6273(00)00159-8, PMID: 11163272

Schrauwen I, Helfmann S, Inagaki A, Predoehl F, Tabatabaiefar MA, Picher MM, Sommen M, Zazo Seco C, Oostrik J, Kremer H, Dheedene A, Claes C, Fransen E, Chaleshtori MH, Coucke P, Lee A, Moser T, Van Camp G. 2012. A mutation in CABP2, expressed in cochlear hair cells, causes autosomal-recessive hearing impairment. The American Journal of Human Genetics 91:636-645. DOI: https://doi.org/10.1016/j.ajhg.2012. 08.018, PMID: 22981119

Schwarz K, Natarajan S, Kassas N, Vitale N, Schmitz F. 2011. The synaptic ribbon is a site of phosphatidic acid generation in ribbon synapses. Journal of Neuroscience 31:15996-16011. DOI: https://doi.org/10.1523/ JNEUROSCI.2965-11.2011, PMID: 22049442

Sendin G, Bulankina AV, Riedel D, Moser T. 2007. Maturation of ribbon synapses in hair cells is driven by thyroid hormone. Journal of Neuroscience 27:3163-3173. DOI: https://doi.org/10.1523/JNEUROSCI.3974-06.2007, PMID: 17376978

Sheets L, Trapani JG, Mo W, Obholzer N, Nicolson T. 2011. Ribeye is required for presynaptic Ca(V)1.3a channel localization and afferent innervation of sensory hair cells. Development 138:1309-1319. DOI: https://doi.org/ 10.1242/dev.059451, PMID: 21350006

Siksou L, Rostaing P, Lechaire JP, Boudier T, Ohtsuka T, Fejtová A, Kao HT, Greengard P, Gundelfinger ED, Triller A, Marty S. 2007. Three-dimensional architecture of presynaptic terminal cytomatrix. Journal of Neuroscience 27:6868-6877. DOI: https://doi.org/10.1523/JNEUROSCI.1773-07.2007, PMID: 17596435 
Sjostrand FS. 1958. Ultrastructure of retinal rod synapses of the guinea pig eye as revealed by three-dimensional reconstructions from serial sections. Journal of Ultrastructure Research 2:122-170. DOI: https://doi.org/10. 1016/S0022-5320(58)90050-9, PMID: 13631744

Smith CA, Sjostrand FS. 1961. Structure of the nerve endings on the external hair cells of the guinea pig cochlea as studied by serial sections. Journal of Ultrastructure Research 5:523-556. DOI: https://doi.org/10.1016/S0022 5320(61)80025-7. PMID: 13914158

Snellman J, Mehta B, Babai N, Bartoletti TM, Akmentin W, Francis A, Matthews G, Thoreson W, Zenisek D. 2011 Acute destruction of the synaptic ribbon reveals a role for the ribbon in vesicle priming. Nature Neuroscience 14:1135-1141. DOI: https://doi.org/10.1038/nn.2870, PMID: 21785435

Sobkowicz HM, Rose JE, Scott GE, Slapnick SM. 1982. Ribbon synapses in the developing intact and cultured organ of Corti in the mouse. Journal of Neuroscience 2:942-957. PMID: 7097321

Spiwoks-Becker I, Glas M, Lasarzik I, Vollrath L. 2004. Mouse photoreceptor synaptic ribbons lose and regain material in response to illumination changes. European Journal of Neuroscience 19:1559-1571. DOI: https:// doi.org/10.1111/j.1460-9568.2004.03198.x, PMID: 15066152

Strenzke N, Chakrabarti R, Al-Moyed H, Müller A, Hoch G, Pangrsic T, Yamanbaeva G, Lenz C, Pan KT, Auge E, Geiss-Friedlander R, Urlaub H, Brose N, Wichmann C, Reisinger E. 2016. Hair cell synaptic dysfunction, auditory fatigue and thermal sensitivity in otoferlin lle515Thr mutants. The EMBO Journal 35:2519-2535. DOI: https:// doi.org/10.15252/embj.201694564, PMID: 27729456

Strenzke N, Chanda S, Kopp-Scheinpflug C, Khimich D, Reim K, Bulankina AV, Neef A, Wolf F, Brose N, XuFriedman MA, Moser T. 2009. Complexin-l is required for high-fidelity transmission at the endbulb of Held auditory synapse. Journal of Neuroscience 29:7991-8004. DOI: https://doi.org/10.1523/JNEUROSCI.0632-09. 2009, PMID: 19553439

Taberner AM, Liberman MC. 2005. Response properties of single auditory nerve fibers in the mouse. Journal of Neurophysiology 93:557-569. DOI: https://doi.org/10.1152/jn.00574.2004, PMID: 15456804

tom Dieck S, Altrock WD, Kessels MM, Qualmann B, Regus H, Brauner D, Fejtová A, Bracko O, Gundelfinger ED Brandstätter JH. 2005. Molecular dissection of the photoreceptor ribbon synapse: physical interaction of Bassoon and RIBEYE is essential for the assembly of the ribbon complex. The Journal of cell biology 168:825836. DOI: https://doi.org/10.1083/jcb.200408157, PMID: 15728193

Vaithianathan T, Matthews G. 2014. Visualizing synaptic vesicle turnover and pool refilling driven by calcium nanodomains at presynaptic active zones of ribbon synapses. PNAS 111:8655-8660. DOl: https://doi.org/10, 1073/pnas.1323962111, PMID: 24912160

Van Epps HA, Hayashi M, Lucast L, Stearns GW, Hurley JB, De Camilli P, Brockerhoff SE. 2004. The zebrafish nrc mutant reveals a role for the polyphosphoinositide phosphatase synaptojanin 1 in cone photoreceptor ribbon anchoring. Journal of Neuroscience 24:8641-8650. DOI: https://doi.org/10.1523/JNEUROSCI.2892-04.2004, PMID: 15470129

Vogl C, Cooper BH, Neef J, Wojcik SM, Reim K, Reisinger E, Brose N, Rhee JS, Moser T, Wichmann C. 2015. Unconventional molecular regulation of synaptic vesicle replenishment in cochlear inner hair cells. Journal of Cell Science 128:638-644. DOI: https://doi.org/10.1242/jcs.162099, PMID: 25609709

von Gersdorff H, Vardi E, Matthews G, Sterling P. 1996. Evidence that vesicles on the synaptic ribbon of retinal bipolar neurons can be rapidly released. Neuron 16:1221-1227. DOI: https://doi.org/10.1016/S0896-6273(00) 80148-8, PMID: 8663998

Wichmann C, Moser T. 2015. Relating structure and function of inner hair cell ribbon synapses. Cell and Tissue Research 361:95-114. DOI: https://doi.org/10.1007/s00441-014-2102-7, PMID: 25874597

Wittig JH, Parsons TD. 2008. Synaptic ribbon enables temporal precision of hair cell afferent synapse by increasing the number of readily releasable vesicles: a modeling study. Journal of Neurophysiology 100:17241739. DOI: https://doi.org/10.1152/jn.90322.2008, PMID: 18667546

Wong AB, Rutherford MA, Gabrielaitis M, Pangrsic T, Göttfert F, Frank T, Michanski S, Hell S, Wolf F, Wichmann C, Moser T. 2014. Developmental refinement of hair cell synapses tightens the coupling of $\mathrm{Ca} 2+$ influx to exocytosis. The EMBO Journal 33:n/a-264. DOI: https://doi.org/10.1002/embj.201387110, PMID: 24442635 Yang PS, Alseikhan BA, Hiel H, Grant L, Mori MX, Yang W, Fuchs PA, Yue DT. 2006. Switching of Ca2+dependent inactivation of $\mathrm{Ca}(\mathrm{v}) 1.3$ channels by calcium binding proteins of auditory hair cells. Journal of Neuroscience 26:10677-10689. DOI: https://doi.org/10.1523/JNEUROSCI.3236-06.2006, PMID: 17050707 Zampini V, Johnson SL, Franz C, Lawrence ND, Münkner S, Engel J, Knipper M, Magistretti J, Masetto S,

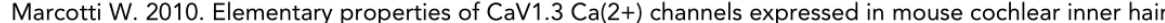
cells. The Journal of Physiology 588:187-199. DOI: https://doi.org/10.1113/jphysiol.2009.181917, PMID: 1 9917569

Zenisek D, Horst NK, Merrifield C, Sterling P, Matthews G. 2004. Visualizing synaptic ribbons in the living cell. Journal of Neuroscience 24:9752-9759. DOI: https://doi.org/10.1523/JNEUROSCI.2886-04.2004, PMID: 15525760 
A

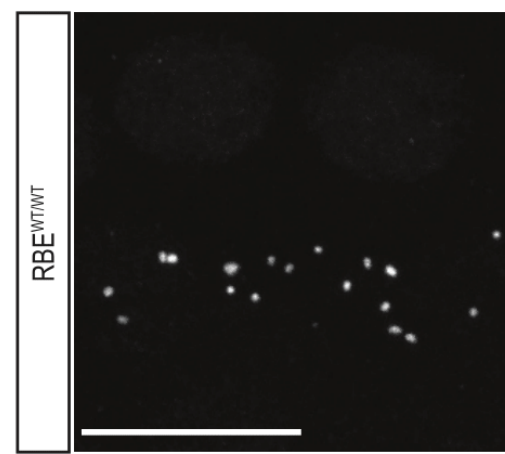

B

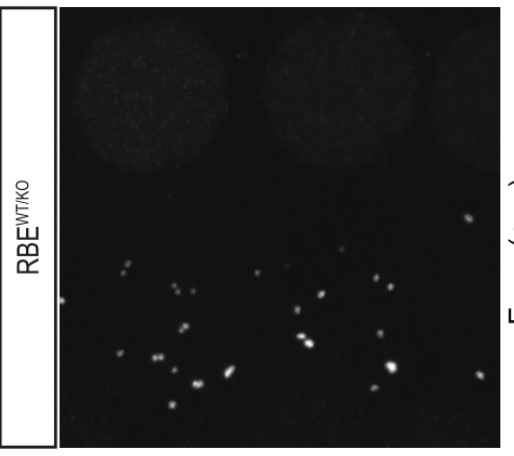

Figure 1-figure supplement 1. Gene-dosage dependent expression of the RIBEYE at IHC AZs. (A) Maximal projection of organs of Cort immunolabeled for the C-terminal domain of both CtBP2 and RIBEYE, present in the nuclei and the ribbons. The number of ribbons does not differ between $\mathrm{RBE}^{\mathrm{WT} / \mathrm{KO}}(n=50 \mathrm{IHCs}, \mathrm{N}=4)$ and RBE ${ }^{\mathrm{WT}} / \mathrm{WT} \mathrm{IHCs}(n=39 \mathrm{IHCs}, \mathrm{N}=3)$. Scale bar $=10 \mu \mathrm{m}$. (B) Box plot highlighting significantly reduced immunofluorescence intensities for the ribbons in the heterozygous condition ( $n=600$ spots for $40 \mathrm{RBE}^{\mathrm{WT} / \mathrm{KO}} \mathrm{HCs}, \mathrm{N}=3$ and $n=411$ spots for 29 RBE ${ }^{W T} / W T$ IHCs, $N=3, p<0.0001$, Mann-Whitney-Wilcoxon test). Box plots show 10, 25, 50, 75 and $90^{\text {th }}$ percentiles with individual immunofluorescent spots overlaid; means are shown as crosses.

DOI: https://doi.org/10.7554/eLife.29275.004 


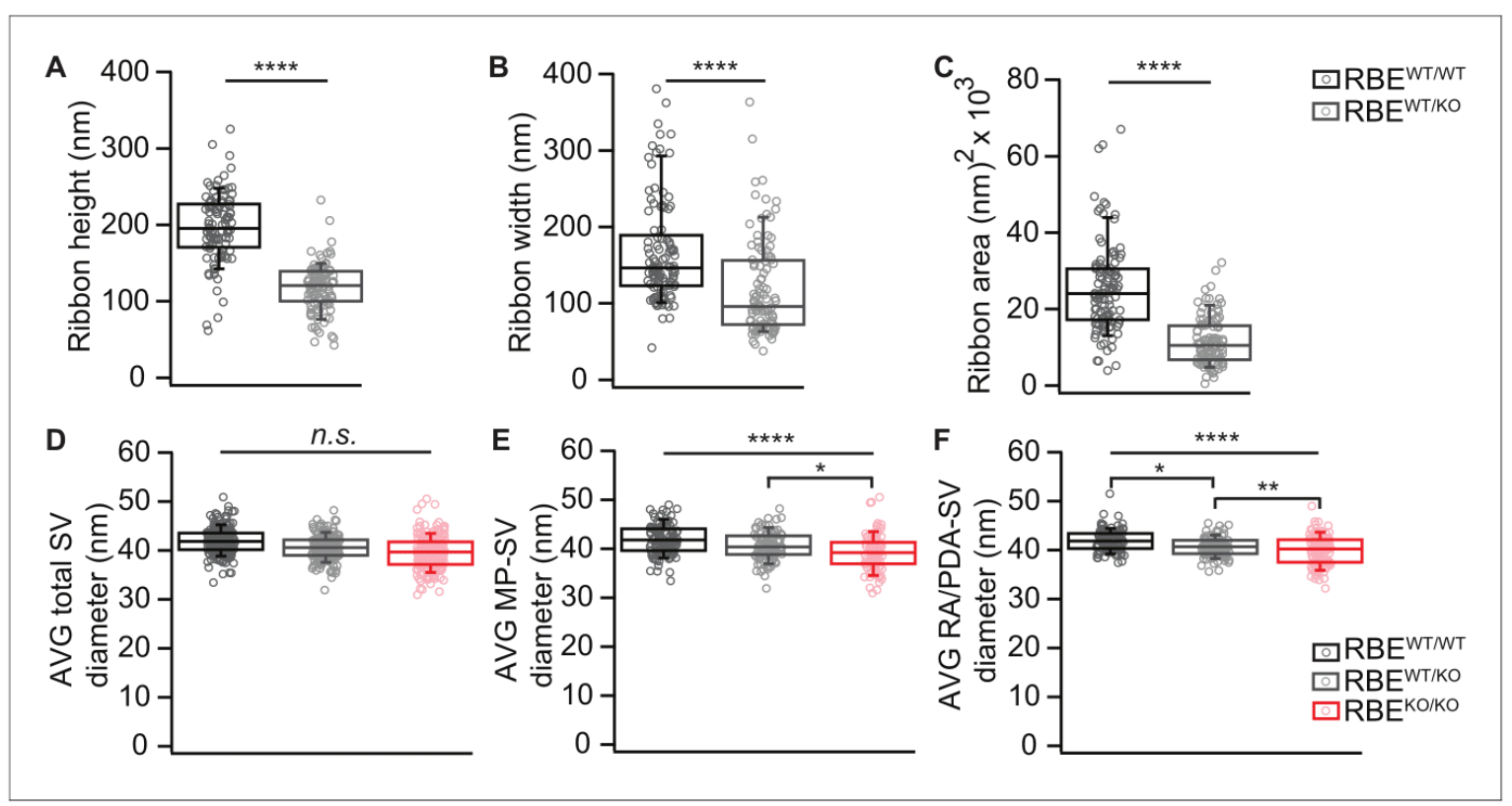

Figure 2-figure supplement 1. Random section analysis showed smaller synaptic ribbons and vesicles in RBE ${ }^{W T / K O}$ mice. (A-C) Random section analysis of P21 mice revealed smaller ribbon sizes in $\mathrm{RBE}^{\mathrm{WT} / \mathrm{KO}} \mid \mathrm{HCs}\left(n=101\right.$ ribbons, $\mathrm{N}=2$ for $\mathrm{RBE}^{\mathrm{WT} / \mathrm{KO}}, n=106$ ribbons, $\mathrm{N}=2$ for $\mathrm{RBE}^{\mathrm{WT}}$ WT ; $p<0.0001$, Mann-Whitney-Wilcoxon test for all), while the SV diameter per ribbon/PD was unaltered for all three genotypes considering all SVs (D): $n=108 \mathrm{AZs}, \mathrm{N}=2$ for RBE ${ }^{\mathrm{KO} / \mathrm{KO}}, \mathrm{n}=101 \mathrm{AZs}, \mathrm{N}=2$ for RBE ${ }^{\mathrm{WT} / \mathrm{KO}}, \mathrm{n}=106 \mathrm{AZs}, \mathrm{N}=2$ for RBE ${ }^{\mathrm{WT} / \mathrm{WT}}$ as for (E, F); $\mathrm{p}=0.30, \mathrm{NPMC}$ test. However, a reduction of MP-SV diameter (E): RBE ${ }^{\mathrm{KO} / K O}$ and RBE ${ }^{\mathrm{WT} / \mathrm{KO}}$ vs. RBE ${ }^{\mathrm{WT} / \mathrm{WT}} ; \mathrm{p}<0.0001, \mathrm{NPMC}$ test and RBE ${ }^{\mathrm{WT} / K O} \mathrm{vs}$. RBE ${ }^{\mathrm{KO} / K O} ; \mathrm{p}=0.03, \mathrm{NPMC}$ test) and

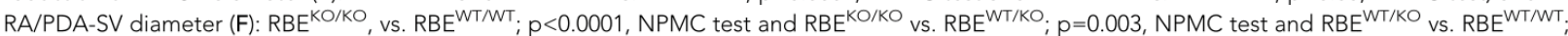
$p=0.02, N P M C$ test) was observed for RBE ${ }^{W T / K O}$ and $R_{B E}{ }^{K O} / K O$. Box plots show $10,25,50,75$ and $90^{\text {th }}$ percentiles with individual data points overlaid. DOI: https://doi.org/10.7554/eLife.29275.006 
A Tomogram analysis modified B

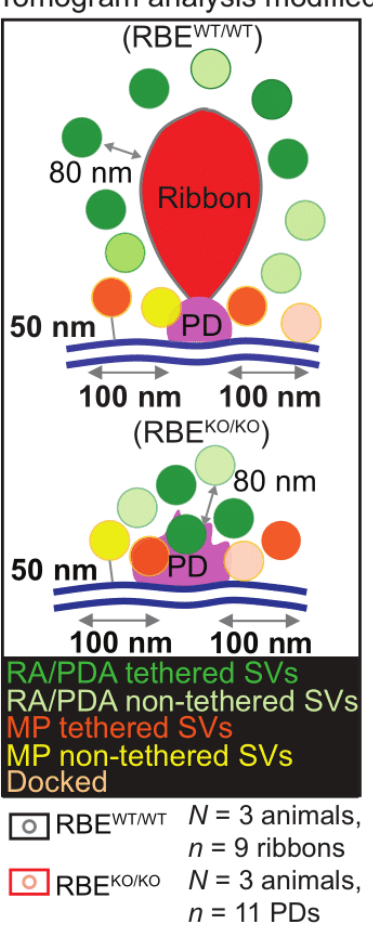

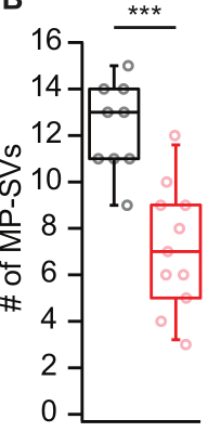

C

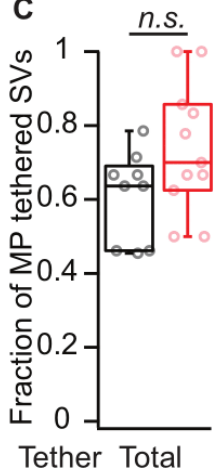

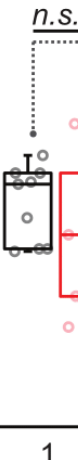

.S. $* * * *$

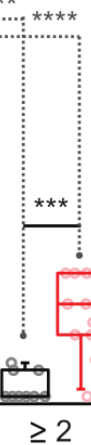

D

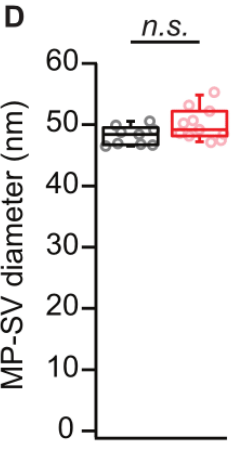

E

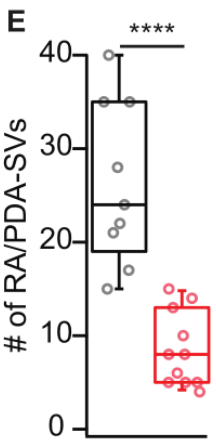

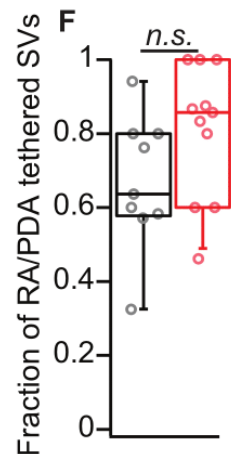

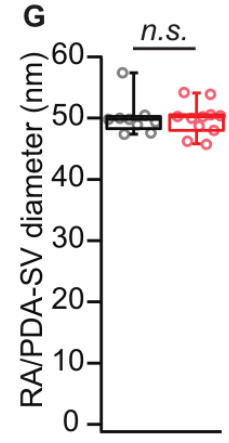

Figure 3-figure supplement 1. Electron tomogram analysis according to Jung et al., 2015a. (A) Illustrations for tomogram analysis parameters, as used in Jung et al., 2015a are depicted. The MP-SVs were defined as vesicles with $\leq 50 \mathrm{~nm}$ from the AZ membrane and with the shortest distance from the vesicle membrane to the presynaptic density of $\leq 100 \mathrm{~nm}$, excluding the RA/PDA-SVs. (B-D) Quantification for MP-SVs: $n=11$ PDs, $N=3$ animals for $\mathrm{RBE}^{\mathrm{KO} / \mathrm{KO}}$ and $n=9$ ribbons, $\mathrm{N}=3$ animals for RBE ${ }^{\mathrm{WT}}$ WT were analyzed. Fewer MP-SVs were observed in RBE ${ }^{\mathrm{KO} / K O}$ (B; $\mathrm{P}=0.0001, \mathrm{Mann}-\mathrm{Whitney-}$ Wilcoxon test). The fraction of tethered MP-SVs was unaltered in $\mathrm{RBE}^{\mathrm{KO} / \mathrm{KO}}$ ( $p=0.06$, t-test). Most of the SVs were tethered by a single tether in both $\mathrm{RBE}^{\mathrm{WT} / W T}$ and RBE ${ }^{\mathrm{KO} / \mathrm{KO}}$. Significantly more multiple-tethered SVs were observed in RBE ${ }^{\mathrm{KO} / K O}$ (C; single-tethered MP-SVs; multiple-tethered MP-SVs, $n$. s.: $p>0.05, * \star * \star: ~ p<0.0001$, Tukey's test). MP-SV diameters were unaltered in RBE ${ }^{K O / K O}$ ( $D ; p=0.06$, Mann-Whitney-Wilcoxon test). (E-G) Quantification for RA/PDA-SVs (same sample size): Significantly fewer PDA-SVs were observed in RBE ${ }^{K O / K O}$ than RA-SVs in RBE ${ }^{\text {WTMT }}$ (E; $P<0.0001, M^{2}$ Mann-WhitneyWilcoxon test). The fraction of tethered PDA-SVs in RBE ${ }^{K O / K O}$ was comparable to that of tethered RA-SVs in RBEWT/WT (F; $\left.P=0.10, t-t e s t\right)$. RA/PDA-SV diameters were unaltered (G; $p=0.68$, Mann-Whitney-Wilcoxon test). Box plots show 10, 25, 50,75 and $90^{\text {th }}$ percentiles with individual data points overlaid.

DOI: https://doi.org/10.7554/eLife.29275.008 
A
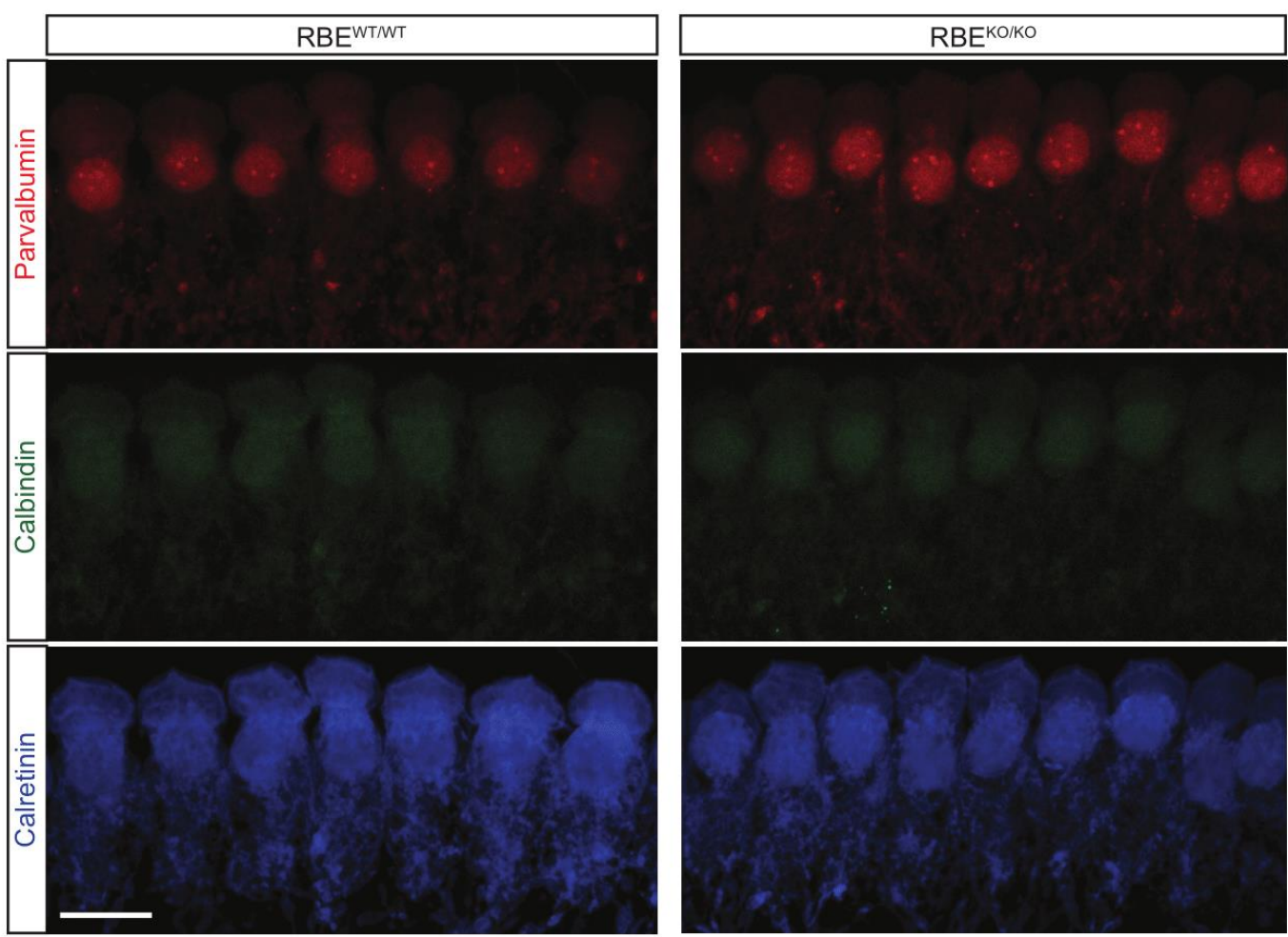

B

Parvalbumin

Calbindin

Calretinin
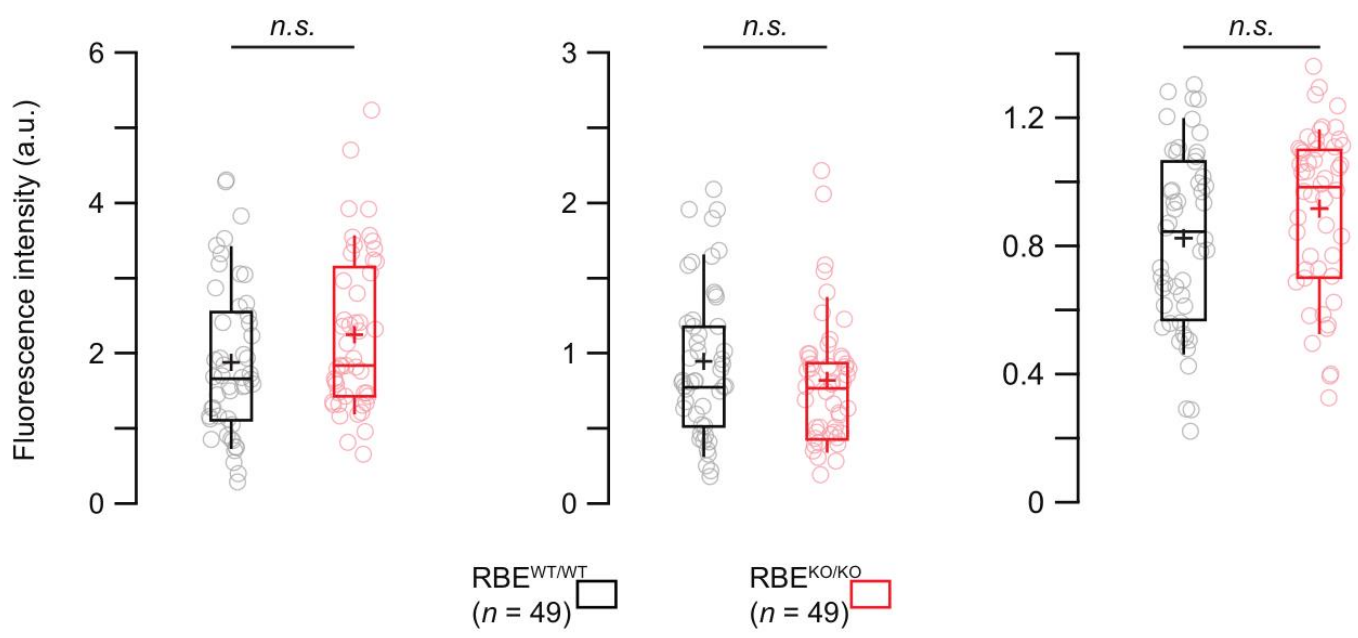

Figure 6-figure supplement 1. Semi-quantitative immunofluorescence analysis of the three main proteinaceous $\mathrm{Ca}^{2+}$-buffers. (A) Maximal projection of confocal sections from organs of Corti co-immunolabeled for parvalbumin (top row), calbindin (middle row) and calretinin (bottom row). Scale Figure 6-figure supplement 1 continued on next page 


\section{eLIFE}

Research article

Figure 6-figure supplement 1 continued

bar $=10 \mu \mathrm{m}$. (B) Box plots showed no significant differences in immunofluorescence intensities for the three proteinaceous Ca ${ }^{2+}$-buffers between $\mathrm{RBE}^{\mathrm{KO} / \mathrm{KO}}$ and $\mathrm{RBE}{ }^{\mathrm{WT} / \mathrm{WT}}$ conditions $(n=49 \mathrm{IHCs}, \mathrm{N}=4$ for both genotypes, $\mathrm{p}=0.08,0.23$ and 0.09 from left to right, Mann-Whitney-Wilcoxon test for all). Box plots show $10,25,50,75$ and $90^{\text {th }}$ percentiles with individual cells overlaid; means are shown as crosses

DOI: https://doi.org/10.7554/eLife.29275.012 


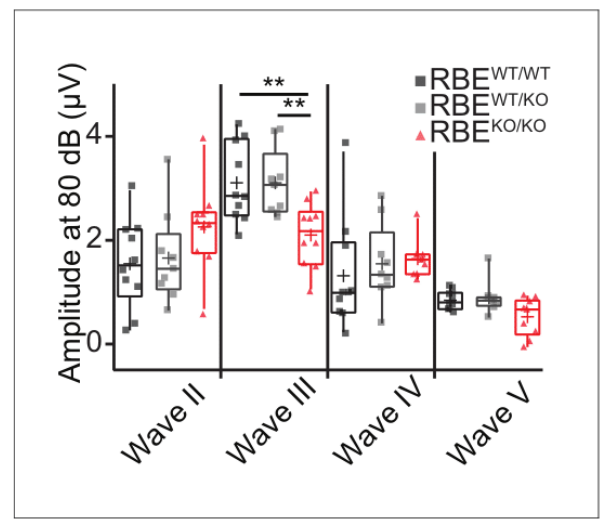

Figure 8-figure supplement 1. Quantification of $A B R$ waves $\mathrm{II}-\mathrm{V}$ amplitude in $\mathrm{RBE}{ }^{\mathrm{KO} / \mathrm{KO}}$ mice. Mean $\mathrm{ABR}$ wave II-V amplitude in response to an $80 \mathrm{~dB}$ click in $6 / 8$ week-old mice. Responses were comparable in waves II, IV and V (Tukey's test for comparison of the three groups regarding wave II and III, NPCM test for comparison of wave $I V$ and $V, n=N=10$ for RBE ${ }^{W T M}$ and $\mathrm{RBE}^{\mathrm{KO} / \mathrm{KO}}, \mathrm{n}=\mathrm{N}=9$ for $\mathrm{RBE}^{\mathrm{WT} / \mathrm{KO}}$ ), but significantly reduced in wave III in $\mathrm{RBE} \mathrm{KO}^{\mathrm{K} O}$ mice as compared to $\mathrm{RBE}^{\mathrm{WT} / \mathrm{WT}}$ and $\mathrm{RBE}^{\mathrm{WT} / \mathrm{KO}}$ ( $\mathrm{p}=0.0093$ and $p=0.0075$, respectively). Box plots show $10,25,50,75$ and $90^{\text {th }}$ percentiles with individual data points overlaid and means are shown as crosses.

DOI: https://doi.org/10.7554/eLife.29275.015 
A

$$
\begin{aligned}
& \square \operatorname{RBE}^{\text {WTNTT }}(n=19) \\
& \square \operatorname{RBE}^{\text {KO } / \text { KO }}(n=24)
\end{aligned}
$$

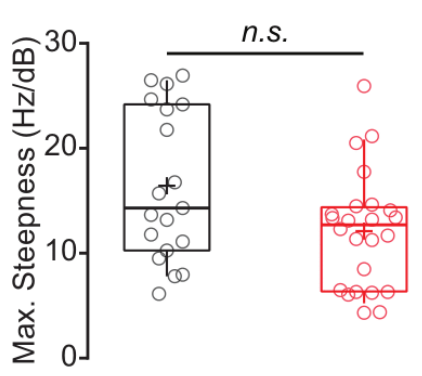

B

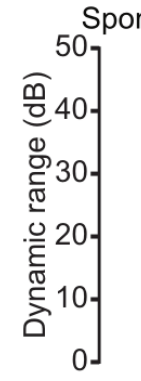

C

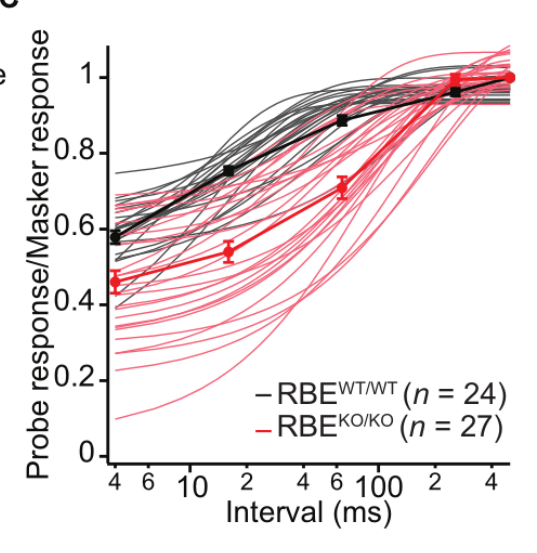

Figure 11-figure supplement 1. Rate-level functions and dynamic range remained unchanged in RBE ${ }^{\mathrm{KO} / \mathrm{KO}}$. (A) Maximal steepness of the rate-level functions (average increase of spike rate with sound intensity) were comparable in RBE ${ }^{\mathrm{KO} / \mathrm{KO}}$ ( $\left.n=24 \mathrm{SGNs}, \mathrm{N}=8\right)$ and RBE ${ }^{\mathrm{WT} / \mathrm{WT}}$ ( $n=19 \mathrm{SGNs}$, N = 7) $\left(p=0.0679\right.$, Mann-Whitney-Wilcoxon test). Box plots show 10, 25, 50, 75 and $90^{\text {th }}$ percentiles and means are shown as crosses, as for (B). (B) Dynamic range is defined as the range of sound intensity levels in which the rate-level function shows a spike rate increase between $10 \%$ and $90 \%$ of the difference between spontaneous and maximal rate. The absence of ribbon in RBE ${ }^{\mathrm{KO} / \mathrm{KO}}$ did not affect this estimate significantly ( $p=0.3044$, $t$-test). (C) Ratio of probe and masker onset response (number of spikes during first $10 \mathrm{~ms}$, mean \pm S.E.M.) and single exponential fits to individual units (RBE ${ }^{\mathrm{KO} /}$ $\mathrm{KO}, \mathrm{n}=27 \mathrm{SGNs}, \mathrm{N}=8$, pink and RBE ${ }^{\mathrm{WT} / W T}, \mathrm{n}=24 \mathrm{SGNs}, \mathrm{N}=7$, gray) showed a slower recovery in $\mathrm{RBE}^{\mathrm{KO} / \mathrm{KO}}$ with a significantly lower time constant $(\mathrm{p}<0.0001$, Mann-Whitney-Wilcoxon test).

DOI: https://doi.org/10.7554/eLife.29275.020 
Supplementary file 1

\begin{tabular}{|c|c|c|c|c|c|}
\hline 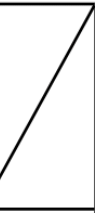 & Parameters & $\begin{array}{c}\text { RBE }^{\mathrm{WT} / \mathrm{WT}} \\
\text { ( } n=9 \text { ribbons, } \\
\mathrm{N}=3 \text { animals) } \\
\text { [mean } \pm \text { (S.E.M.), } \\
\text { (S.D.)] }\end{array}$ & $\begin{array}{c}\text { RBE }^{\mathrm{KO} / \mathrm{KO}} \\
(n=11 \mathrm{PDs}, \\
\mathrm{N}=3 \text { animals) } \\
\text { [mean } \pm \text { (S.E.M.), } \\
\text { (S.D.)] }\end{array}$ & $p$-values & $\begin{array}{l}\text { Statistical } \\
\text { tests }\end{array}$ \\
\hline \multirow{6}{*}{ 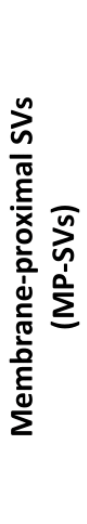 } & SV number & $12.33 \pm(0.65),(1.93)$ & $7.18 \pm(0.81),(2.71)$ & 0.0001 & $\begin{array}{c}\text { Mann- } \\
\text { Whitney- } \\
\text { Wilcoxon } \\
\text { test }\end{array}$ \\
\hline & $\begin{array}{l}\text { Fraction of tethered } \\
\text { SVs }\end{array}$ & $0.61 \pm(0.04),(0.12)$ & $0.74 \pm(0.05),(0.17)$ & 0.06 & t-test \\
\hline & $\begin{array}{l}\text { Fraction of SVs with } \\
\text { single tether }\end{array}$ & $0.58 \pm(0.03),(0.10)$ & $0.51 \pm(0.60),(0.19)$ & 0.64 & \multirow{3}{*}{$\begin{array}{l}\text { Tukey's } \\
\text { test }\end{array}$} \\
\hline & $\begin{array}{l}\text { Fraction of SVs with } \\
\text { multiple tethers }\end{array}$ & $0.03 \pm(0.01),(0.04)$ & $0.22 \pm(0.03),(0.11)$ & 0.008 & \\
\hline & $p$-values & $4.87 E-10$ & $4.21 E-05$ & $\infty$ & \\
\hline & Diameter of SVs & $48.20 \pm(0.51),(1.52)$ & $50.05 \pm(0.80),(2.57)$ & 0.06 & t-test \\
\hline \multirow{3}{*}{ 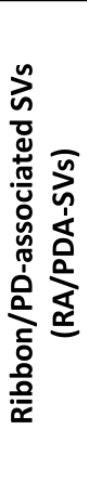 } & SV number & $26.33 \pm(2.30),(8.71)$ & $8.45 \pm(1.20),(3.98)$ & $\begin{array}{c}3.005 E- \\
05\end{array}$ & $\begin{array}{c}\text { Mann- } \\
\text { Whitney- } \\
\text { Wilcoxon } \\
\text { test }\end{array}$ \\
\hline & $\begin{array}{l}\text { Fraction of tethered } \\
\text { SVs }\end{array}$ & $0.70 \pm(0.06),(0.18)$ & $0.80 \pm(0.05),(0.18)$ & 0.10 & t-test \\
\hline & Diameter of SVs & $50.07 \pm(1.00),(2.93)$ & $49.82 \pm(0.80),(2.67)$ & 0.68 & $\begin{array}{c}\text { Mann- } \\
\text { Whitney- } \\
\text { Wilcoxon } \\
\text { test }\end{array}$ \\
\hline
\end{tabular}

\section{Supplementary file 1: Modified tomogram analysis}

The table depicts the modified tomogram analysis performed according to Jung et al. (2015a) displaying the mean \pm (S.E.M.), (S.D.), $p$-values, sample size and statistical tests used to compare RBE ${ }^{W T / W T}$ and $\mathrm{RBE}^{\mathrm{KO} / \mathrm{KO}}$. Refer to Figure 3-figure supplement 1 for the graphs. 
Supplementary file 2

\begin{tabular}{|c|c|c|c|c|c|c|c|c|c|}
\hline & \multicolumn{2}{|c|}{$\mathbf{R}_{\mathrm{s}}$} & \multicolumn{2}{|c|}{$\mathbf{R}_{\mathrm{m}}$} & \multicolumn{2}{|c|}{$I_{\text {leak }}$} & \multicolumn{2}{|c|}{$\mathrm{C}_{\text {slow }}$} \\
\hline & & $\mathrm{RBE}^{\mathrm{WT} / \mathrm{WT}}$ & $\mathrm{RBE}^{\mathrm{KO} / \mathrm{KO}}$ & $\mathrm{RBE}^{\mathrm{WT} / \mathrm{WT}}$ & $\mathrm{RBE}^{\mathrm{KO} / \mathrm{KO}}$ & $\mathrm{RBE}^{\mathrm{WT} / \mathrm{WT}}$ & $\mathrm{RBE}^{\mathrm{KO} / \mathrm{KO}}$ & $\mathrm{RBE}^{\mathrm{WT} / \mathrm{WT}}$ & $\mathrm{RBE}^{\mathrm{KO} / \mathrm{KO}}$ \\
\hline \multirow{2}{*}{$\begin{array}{c}\text { Figure } \\
5\end{array}$} & $\begin{array}{c}\text { ruptured } \\
(A, B, C)\end{array}$ & $\begin{array}{c}11.34 \pm \\
0.89 \mathrm{M} \Omega\end{array}$ & $\begin{array}{c}10.47 \pm \\
0.87 \mathrm{M} \Omega\end{array}$ & $\begin{array}{r}437 \pm \\
55 \mathrm{M} \Omega\end{array}$ & $\begin{array}{c}535 \pm \\
168 \mathrm{M} \Omega\end{array}$ & $\begin{array}{l}-21.19 \pm \\
2.50 \mathrm{pA}\end{array}$ & $\begin{array}{l}-19.43 \pm \\
1.73 \mathrm{pA}\end{array}$ & $\begin{array}{l}11.81 \pm \\
0.28 p F\end{array}$ & $\begin{array}{l}12.53 \pm \\
0.28 \mathrm{pF}\end{array}$ \\
\hline & $\begin{array}{l}\text { perforated } \\
\text { (D) }\end{array}$ & $\begin{array}{c}29.33 \pm \\
2.04 \mathrm{M} \Omega\end{array}$ & $\begin{array}{c}27.22 \pm \\
1.85 \mathrm{M} \Omega\end{array}$ & $\begin{array}{c}1598 \pm \\
184 \mathrm{M} \Omega\end{array}$ & $\begin{array}{l}1532 \pm \\
79 \mathrm{M} \Omega\end{array}$ & $\begin{array}{c}-8 \pm \\
1.04 \mathrm{pA}\end{array}$ & $\begin{array}{l}-7.44 \pm \\
0.74 \mathrm{pA}\end{array}$ & $\begin{array}{l}12.19 \pm \\
0.53 \mathrm{pF}\end{array}$ & $\begin{array}{l}12.76 \pm \\
0.27 \mathrm{pF}\end{array}$ \\
\hline $\begin{array}{c}\text { Figure } \\
6 \\
\end{array}$ & ruptured & $\begin{array}{c}13.75 \pm \\
0.92 \mathrm{M} \Omega\end{array}$ & $\begin{array}{c}13.96 \pm \\
0.82 \mathrm{M} \Omega\end{array}$ & $\begin{array}{r}400 \pm \\
46 \mathrm{M} \Omega\end{array}$ & $\begin{array}{r}406 \pm \\
79 \mathrm{M} \Omega\end{array}$ & $\begin{array}{c}-28 \pm \\
3.73 \mathrm{pA}\end{array}$ & $\begin{array}{c}-26 \pm \\
2.66 \mathrm{pA}\end{array}$ & $\begin{array}{l}10.63 \pm \\
0.29 \mathrm{pF}\end{array}$ & $\begin{array}{l}10.88 \pm \\
0.26 \mathrm{pF}\end{array}$ \\
\hline \multirow{4}{*}{$\begin{array}{c}\text { Figure } \\
7\end{array}$} & $\begin{array}{c}\text { perforated } \\
\text { (A-C) }\end{array}$ & $\begin{array}{c}28 \pm \\
2.23 \mathrm{M} \Omega\end{array}$ & $\begin{array}{c}25.11 \pm \\
1.66 \mathrm{M} \Omega\end{array}$ & $\begin{array}{l}1373 \pm \\
44 \mathrm{M} \Omega\end{array}$ & $\begin{array}{l}1510 \pm \\
76 \mathrm{M} \Omega\end{array}$ & $\begin{array}{r}-7.67 \pm \\
0.69 \mathrm{pA}\end{array}$ & $\begin{array}{c}-8 \pm \\
0.81 \mathrm{pA}\end{array}$ & $\begin{array}{l}11.93 \pm \\
0.49 \mathrm{pF}\end{array}$ & $\begin{array}{l}12.76 \pm \\
0.29 \mathrm{pF}\end{array}$ \\
\hline & $\begin{array}{l}\text { perforated } \\
\text { (D) }\end{array}$ & $\begin{array}{c}23.51 \pm \\
0.91 \mathrm{M} \Omega\end{array}$ & $\begin{array}{c}22.08 \pm \\
0.94 \mathrm{M} \Omega\end{array}$ & $\begin{array}{r}879 \pm \\
51 \mathrm{M} \Omega \\
\end{array}$ & $\begin{array}{r}909 \pm \\
40 \mathrm{M} \Omega\end{array}$ & $\begin{array}{l}-16.54 \pm \\
1.08 \mathrm{pA}\end{array}$ & $\begin{array}{l}-16.67 \pm \\
1.56 \mathrm{pA}\end{array}$ & $\begin{array}{c}9.04 \pm \\
0.17 \mathrm{pF}\end{array}$ & $\begin{array}{c}9.94 \pm \\
0.20 \mathrm{pF}\end{array}$ \\
\hline & $\begin{array}{c}\text { perforated } \\
\text { (E-F) }\end{array}$ & $\begin{array}{c}19.39 \pm \\
1.58 \mathrm{M} \Omega\end{array}$ & $\begin{array}{c}18.67 \pm \\
1.17 \mathrm{M} \Omega\end{array}$ & $\begin{array}{c}1387 \pm \\
138 \mathrm{M} \Omega\end{array}$ & $\begin{array}{l}1226 \pm \\
76 \mathrm{M} \Omega\end{array}$ & $\begin{array}{l}-28.02 \pm \\
2.41 \mathrm{pA}\end{array}$ & $\begin{array}{l}-28.11 \pm \\
2.12 \mathrm{pA}\end{array}$ & $\begin{array}{c}9.13 \pm \\
0.18 \mathrm{pF}\end{array}$ & $\begin{array}{c}9.57 \pm \\
0.45 \mathrm{pF}\end{array}$ \\
\hline & $\begin{array}{c}\text { perforated } \\
\text { (G-H) }\end{array}$ & $\begin{array}{c}18.16 \pm \\
1.30 \mathrm{M} \Omega\end{array}$ & $\begin{array}{c}18.61 \pm \\
1.35 \mathrm{M} \Omega\end{array}$ & $\begin{array}{c}1260 \pm \\
121 \mathrm{M} \Omega\end{array}$ & $\begin{array}{l}1031 \pm \\
62 \mathrm{M} \Omega\end{array}$ & $\begin{array}{l}-23.80 \pm \\
2.46 \mathrm{pA}\end{array}$ & $\begin{array}{r}-21.54 \pm \\
2.70 \mathrm{pA}\end{array}$ & $\begin{array}{c}9.18 \pm \\
0.17 \mathrm{pF}\end{array}$ & $\begin{array}{c}9.32 \pm \\
0.16 \mathrm{pF}\end{array}$ \\
\hline
\end{tabular}

Supplementary file 2: Passive electrical properties of patch-clamp recording experiments

The table shows the mean \pm (S.E.M.) of the passive electrical properties across all ruptured and perforated patch-clamp recording experiments in $\mathrm{RBE}^{\mathrm{WT} / \mathrm{WT}}$ and $\mathrm{RBE}^{\mathrm{KO} / \mathrm{KO}}$ conditions. 
4 Chapter 2: Article in preparation

\title{
"Pou4f1 defines a subgroup of Type I spiral ganglion neurons and is necessary for normal inner hair cell presynaptic $\mathrm{Ca}^{2+}$ signaling"
}

\author{
H. E. Sherrill*, P. Jean*, E.C. Driver, T. Moser, and M.W. Kelley
}

* Equal contributions

\section{Author Contributions:}

H.E.S and E.C.D performed immunostainings and analysis and contributed to the manuscript. P.J. performed patch-clamp and $\mathrm{Ca}^{2+}$ imaging, analyzed morphological and physiological data and contributed to the preparation of the manuscript. T.M. initiated and planned project with P.J., H.E.S. and M.W.K, supervised P.J, discussed data and contributed to manuscript preparation. 


\begin{abstract}
Acoustic signals are relayed from the ear to the brain via spiral ganglion neurons (SGNs) that receive auditory information from the cochlear inner hair cells and transmit that information to the cochlear nucleus of the brainstem. Physiologically distinct classes of SGNs have been characterized by their spontaneous firing rate, and those physiological distinctions correspond to stereotyped synaptic positions on the inner hair cell (IHC). More recently, single cell profiling has identified multiple groups of SGNs based on transcriptional profiling, however, correlations between any of these groups and distinct neuronal physiology has not been determined.In this study, we show that expression of the POU transcription factor Pou4f1 in Type I SGNs correlates with a synaptic location on the modiolar side of IHCs. Conditional deletion of Pou4f1 in SGNs beginning at embryonic day 13 rescues the early pathfinding and apoptotic phenotypes reported for germline deletion of Pou4fl, resulting in a phenotypically normal development of SGN patterning. However, conditional deletion of Pouff1 in SGNs alters the activation of $\mathrm{Ca}^{2+}$-channels in IHCs, primarily by increasing their voltage-sensitivity. Moreover, the modiolar to pillar gradient of active zone (AZ) $\mathrm{Ca}^{2+-}$ influx strength is eliminated. These results demonstrate that a subset of SGNs retain expression of Pou4f1 beyond the onset of hearing and suggest that this transcription factor plays an instructive role in presynaptic $\mathrm{Ca}^{2+}$ signaling in IHCs.
\end{abstract}

\title{
Significance statement
}

Physiologically distinct classes of Type I spiral ganglion neurons (SGNs) are necessary to faithfully encode sound intensities spanning the entire audible range. While anatomical studies have demonstrated structural correlates for some physiologically-defined classes of Type I SGNs, an understanding of the molecular pathways that specify each of these types is only emerging. Here, we demonstrate that the continued expression of the homeodomain transcription factor Pou4f1 in type I SGNs corresponds to a distinct subgroup of SGNs that synapse on the medial side of cochlear inner hair cells. This synaptic location has previously been correlated with low spontaneous rate fibers. The conditional deletion of Pou4fl after SGN formation does not disrupt ganglion size or morphology, change the distribution of synaptic locations on IHCs, or impact the creation of synapses, but does influence the voltagedependence and strength of $\mathrm{Ca}^{2+}$-influx at presynaptic AZs in IHCs. 


\section{Introduction}

In mammals, hearing begins with the conversion of pressure waves into electro-chemical signals within auditory inner hair cells (IHCs) located in the cochlea. This signal is then relayed to the brain via Type I spiral ganglion neurons (SGNs). In order to encode a broad dynamic range of sounds, multiple categories of Type I SGNs, each with distinct physiological properties, innervate each IHC. While the full extent of SGN diversity is poorly understood, individual neurons can be classified based on their spontaneous firing rate, threshold of activation and more recently, transcriptional profile (Petitpre et al., 2018; Shrestha et al., 2018; Sun et al., 2018). Based on physiological properties, three Type I SGNs, high-spontaneous rate, medium spontaneous rate and low spontaneous rate, have been described in several species (Liberman, 1978; Winter et al., 1990; Taberner and Liberman, 2005). In addition, the firing properties of individual SGNs correlate with the location of each cell's synapse on the base of an IHC. SGNs with low spontaneous rates and high thresholds are located on the medial (modiolar) sides of IHCs opposing active zones (AZs) with larger and, in some instances, multiple synaptic ribbons, stronger maximal $\mathrm{Ca}^{2+}$ influx that operates in a more depolarized range. In contrast, neurons with high spontaneous rates and low thresholds typically oppose smaller AZs with weaker but more hyperpolarized operating $\mathrm{Ca}^{2+}$ influx, located on the lateral (pillar) sides of IHCs (Winter et al., 1990; Ohlemiller et al., 1991; Merchan-Perez and Liberman, 1996; Taberner and Liberman, 2005; Meyer et al., 2009; Ohn et al., 2016). Hence, it was hypothesized that the IHC decomposes sound intensity information into these different neural channels via diversifying the operating range of the $\mathrm{Ca}^{2+}$ influx among the AZs (Ohn et al., 2016; reviewed in Pangrsic et al., 2018). However, the mechanisms setting up the differences of the presynaptic AZs remained to be elucidated and the proposed link to SGN properties requires experimental testing. For example, it is of great interest to ask whether SGNs can instruct presynaptic properties according to their neural identity.

Recently, single cell RNAseq has identified at least three categories of Type I SGNs based on transcriptional expression profiles (Shrestha et al., 2018; Sun et al., 2018; Petitpre et $a l ., 2018)$. While the molecular profiles for each of these groups are distinct, there is currently no data to confirm the postulated correspondence to any of the three functionally defined SGN groups. Pou4f1, a member of the POU (Pit-Oct-Unc) family of transcription factors was shown to be more strongly expressed in a single class of Type I SGNs in each study. POU transcription factors are a highly studied class of largely neuro-specific molecules that are important for 
neurogenesis, differentiation and survival (Tantin, 2013; Malik et al., 2018). The three members of the POU4F subfamily are expressed in distinct but overlapping populations of neuronal and somatic cells in developing and adult tissues (Jehn et al., 1994; Sajgo et al., 2017). Previous studies have demonstrated that Pou $4 f 1$ is broadly expressed in all SGNs beginning as early as the onset of neurogenesis at E10 (Huang et al., 2001; Deng et al., 2014). Germline deletion of Pou4fl leads to significant pathfinding defects within the entire SGN population and ultimately to a loss of approximately $30 \%$ of all SGNs (Huang et al., 2001). However, transcriptomic and immunological analyses indicate that expression of Pou4f1 begins to be down-regulated in some SGNs as early at E16 with only a limited population of SGNs maintaining expression of Pou4f1 beyond birth (Deng et al., 2014). Given the role of Pou4f1 in cell fate specification, we sought to characterize the morphology and function of those SGNs that maintain expression of Pou4f1 beyond the early post-natal period. Moreover, using morphological and functional analysis of AZ properties in the IHCs of mice with postnatal Pou4f1 disruption, we tested for an instructive trans-synaptic influence of Pou4f1-positive SGNs on their presynaptic input.

\section{Results}

\section{Expression of Pou4f1 is restricted to a subset of SGNs beginning at E16}

Previous studies have suggested that nearly $90 \%$ of all SGNs are positive for Pou $4 \mathrm{fl}$ at P0 (Huang et al., 2001). To determine if a similar pattern of expression was true at the protein level, anti-Pou4f1 immunolabeling was performed on sectioned inner ears from E10.5, E14.5, E16.5, P0, and P14. As previously reported, at E10.5 and E14.5, all, or nearly all, neurons within the $\mathrm{CVG}$ and developing vestibular and spiral ganglia were positive for Pou4f1 (Figure 1). In addition, Pou4f1 was also expressed by a few neurons in the closely associated facial ganglion (Figure 1). In contrast, at E16.5, there was a notable reduction in the number of Pou4f1+ neurons in the basal of the cochlea ( $38 \pm 12 \%$ of total, $n=3)$, while all SGNs in the apex were still positive for Pou4f1 $(100 \pm 0 \%, n=3)$ (Figure 2). The number of Pou4f1 ${ }^{+}$ neurons in the middle turn was intermediate $(51 \pm 12 \%, n=3)$ between basal and apical values, suggesting a development down-regulation of Pou4f1. At P1, the number of Pou4f1+ SGNs was decreased somewhat in the base, dropping to $31 \pm 4 \%,(n=8)$. Similar decreases were observed in the middle turn $(30 \pm 1.2 \%, n=8)$ and in the apex which decreased to $35 \pm 3 \%$ ( $n$ $=8$ ). Finally, at P14, around the onset of hearing, Pou4f1 expression is only present in a total 
of $28 \pm 9 \%(n=5)$ of SGNs however, a slight gradient still appears to persist with more Pou4f1+ cells located in the apical turn. These results are consistent with single cell RNAseq analyses suggesting that approximately $1 / 3$ of SGNs maintain expression of Pou4f1 at adult stages (Shrestha et al., 2018; Sun et al., 2018; Petitpre et al., 2018).

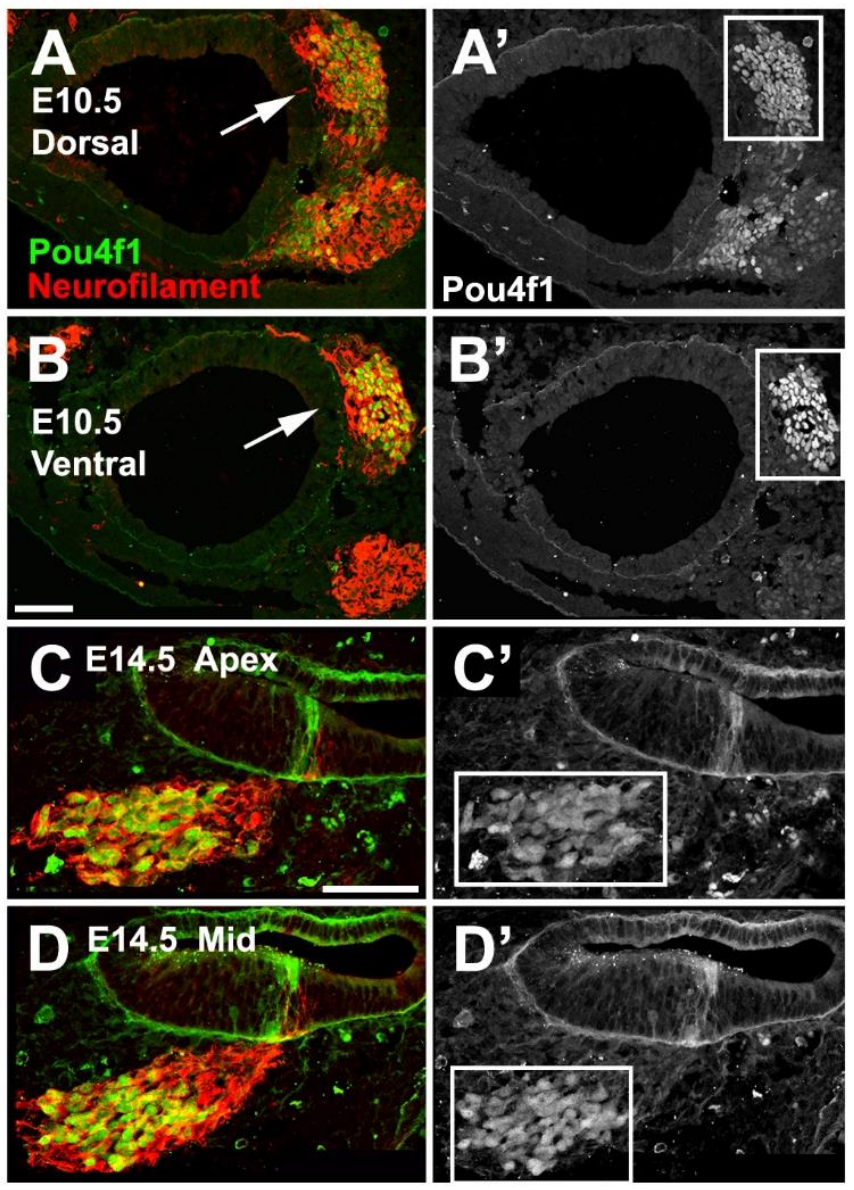

Figure 1. Pou4f1 is broadly expressed in developing CVG and SGNs at early time points (A-B) Expression of Pou4f1 at E10.5. Cross-sections through the dorsal (A) and ventral (B) portions of the otocyst indicate Pou4f1 (green) is initially expressed in most neurons (neurofilament in red) within the CVG (arrows in A,B, boxed in A',B'). (C-D) Mid-modiolar sections through the cochlea at E14.5. Pou4f1 is still broadly expressed in the cell bodies within the developing SG. Panels labeled as in A. Scale bars in A, C $=100 \mu \mathrm{m}$.

\section{Peripheral axons from Pou4f1+ SGNs terminate on the modiolar side of IHCs}

To determine whether Pou4f1 ${ }^{+}$SGNs share any phenotypic characteristics, transgenic Pou4f1 ${ }^{\text {creErt2 }}$ mice (O'Donovan et al., 2014) were combined with either $R 26 R^{\text {zsgreen }}$ or $R 26 R^{\text {tdTomato }}$ reporter mice. Induction of creErt 2 activity was achieved by daily subcutaneous injections of tamoxifen for 3-7 days beginning on P5 (see methods). Results indicated reporter 
expression in over $60 \%$ of SGNs, both Type I and Type II (not shown). This result was surprising given the consistent labeling of approximately $32 \%$ of SGNs using antibodies at the same age and the comparable results obtained by single RNAseq (Shrestha et al., 2018; Sun et al., 2018; Petitpre et al., 2018). Specificity of the antibody used has been confirmed in several previous publications (Blanchard et al., 2015; Hua et al., 2015) and our results indicate a complete loss of antibody labeling in SGNs from Pouf41 mutant mice (see below). To confirm that SGNs expressing the reporter were also positive for Pou4f1, induced cochleae were sectioned and counter-stained for Pou4f1 (Figure 3). A relatively limited number of tdTomato ${ }^{+}$ neurons were also positive for nuclear Pou4f1 (Figure 3). To determine whether the mismatch between reporter activity and Pou4f1 expression could be a result of a down regulation of Pou4f1 between the time of induction and fixation, P0 embryos were induced for three consecutive days and then fixed on the fourth day. Tissue sections indicated a similar mismatch between reporter activity and antibody labeling (not shown). A specific explanation for this discrepancy is unclear but may be related to the method by which the Pou $f f 1^{\text {creErt2 }}$ line was created. The creErt 2 sequence was linked to an $11 \mathrm{~kb}$ Pou $4 f 1$ upstream promoter and then delivered by pronuclear injection (O’Donovan et al 2014). Subsequent analysis of reporter expression in the spinal cord appeared to correspond with the known expression pattern of Pou4f1, but similar examination of reporter expression in the SGN was not determined. Therefore, it is possible that the $11 \mathrm{~kb}$ upstream regulatory sequence lacks regulatory elements that are crucial for accurate SGN expression.

Based on the results described above, we decided to examine the phenotype of Pou $4 \mathrm{fl}^{+} \mathrm{SGNs}$ by combining antibody-labeling with genetic sparse labeling of SGNs using a $N g n 1^{\text {CreERt2 }}$;26R ${ }^{\text {TdTomato }}$ mouse line (Koundakjian et al., 2007; Coate et al., 2015). The $N g n 1^{\text {CreERt2 }} ; 226 R^{\text {TdTomato }}$ labeling strategy results in expression of tdTomato throughout the entire length of individual SGNs. Cochleae were dissected at P3, immuno-labeled with antiPou4f1 antibodies and then prepared as whole mounts. SGN cell bodies that were positive for Pou4f1 and tdTomato were identified and the peripheral projections of those cells were traced to their targets in the organ of Corti (Figure 4). SGNs that were strongly positive for Pou4f1 and tdTomato were found to terminate preferentially on the modiolar side of IHCs (Figure 4). This distribution significantly differed from the distribution of all sparsely-labeled fibers only half of which terminated on the modiolar sides of IHCs (Figure 4). The modiolar localization of synaptic contacts is consistent with the subgroup of Type 1 SGNs that have been described as having a low spontaneous firing rate in the cat (Merchan-Perez and Liberman, 1996). It 
should be noted that while the peripheral axons from nearly all Pou $4 \mathrm{f} 1^{+} \mathrm{SGNs}$ terminate on the modiolar sides of IHCs, it is not the case that every modiolar synapse was formed with a cell that was positive for Pou4f1. This suggests that Pou4f1 defines a subgroup within the larger population of low spontaneous rate fibers.

\section{Base}
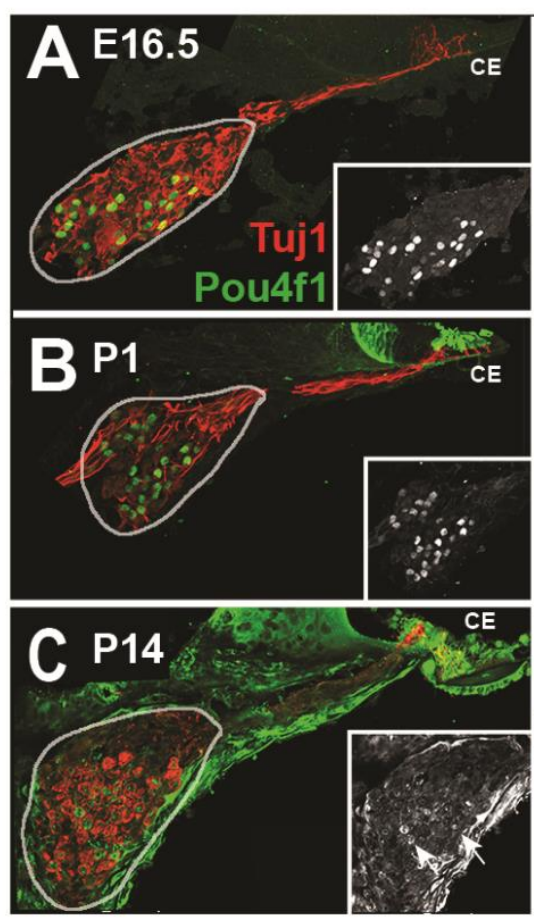

Mid
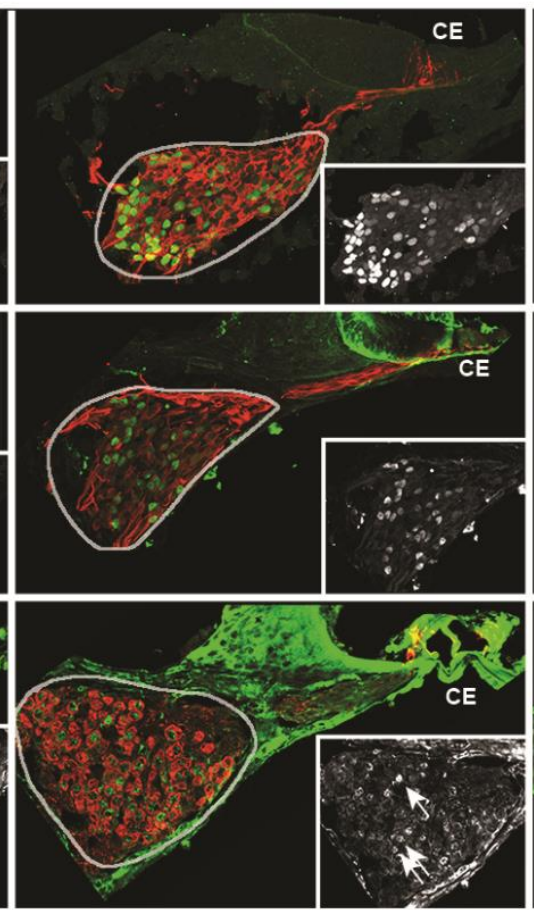

Apex
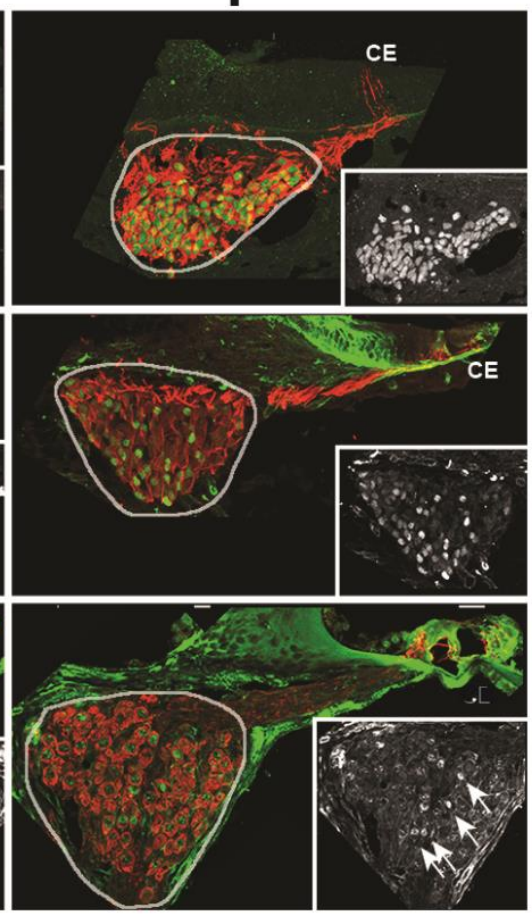

Figure 2. Expression of Pou4f1 changes with development

(A-C) Mid-modiolar cross-sections through the cochlear duct at the indicated time points and cochlear positions. SGNs are labeled with Tuj1 (red) and Pou4f1 (green). For each panel the SG is circled. Insets show expression of Pou4f1 alone in the SG. By comparison with E14.5, the number of SGNs that express Pou4f1 has decreased by E16.5 although a clear basal to apical gradient is present with more Pou4f1+ SGNs in the apex. The number of Pou4f1+ SGNs continues to decrease at both P1 and $\mathrm{P} 14$, but $32 \%$ of SGNs remain positive for Pou4f1 at P14 (arrows). Scale bar in A=100 $\mu \mathrm{m}$. CE= cochlear epithelium

\section{Tissue specific deletion of Pou $4 f 1$ using ectopic Atoh $1^{\text {cre }}$ expression}

To examine the role of Pou4f1 in the subset of low spontaneous rate fibers, we wanted to delay deletion of the gene until after the early period of broad expression in nearly all SGNs (Huang et al., 2001). Initial attempts using Ngn1 ${ }^{\text {creErt2 }}$ yielded either a recapitulation of the phenotype observed in germ line deleted animals, including death of the animal on P0 or no deletion of 
Pou4f1, depending on the dose of tamoxifen used. As discussed above, our results indicated that recombination using the Pou $4 \mathrm{fl}^{\text {creErt2 }}$ line did not reflect the endogenous pattern of Pou $4 \mathrm{f} 1$ expression and, as a result, this line could not be used to reliably delete Pou4fl. Therefore, we decided to take advantage of the known ectopic cre expression in the SG in one of the early Atohl $1^{\text {cre }}$ lines (Matei et al., 2005). This line was generated by placing the Atoh1 3' enhancer upstream of cre (Lumpkin et al., 2003). The resulting transgenic mice show ectopic expression in a number of tissues, including the SG, that are negative for Atohl/Atoh1 based on in situ hybridization, immunolabeling or RNA-seq profiling. Initial transgene expression in the SG begins on E14.5 (data not shown). Atohl $1^{\text {cre }}$ mice were crossed with Pou4ff flox/- mice (Xiang et al., 1996; Badea et al., 2009a; Badea et al., 2009b) to yield Atohl ${ }^{\text {cre }}$;Pou4f $1^{\text {flox/- mice. }}$ Analysis of the SG in Atoh ${ }^{\text {cre }}$;Pou4f $1^{\text {flox/- }}$ mice at E17.5 indicated a nearly complete absence of Pou4f1 expression as compared to controls (Figure 5). By P1, virtually no expression of Pou4f1 was observed in the nuclei of Atoh $1^{\text {cre }} ;$ Pou $4 \mathrm{f} 1^{\text {flox/-mice. }}$.

\section{Cochlear morphology is preserved in Atoh $1^{\mathrm{cre}} ; P$ Pou $4 \mathrm{f1}^{\text {flox/- }}$ mice}

Analysis of cochlear whole mounts from P1 Atoh $1^{\text {cre }} ; P$ Pou $4 \mathrm{f}^{\text {flox/- }}$ and control littermates $\left(\right.$ Atoh $\left.1^{\mathrm{Cre}} ; \mathrm{Pou}_{\mathrm{f}} \mathrm{I}^{-/+}\right)$indicated no differences in the length, size or anatomy of the cochlea (not shown). Cell counts of SGNs in experimental or control cochleae showed no overall change in cell number $(83.8 \pm 5.2$ cells/section $(n=18)$ vs $79.1 \pm 4.5$ cells/section $(n=14) ; p=0.59$, Paired T-test) or density in the ganglion $\left(43 \pm 6\right.$ cells $/ 100 \mu \mathrm{m}^{3}$ vs $46 \pm 6$ cells $/ 100 \mu \mathrm{m}^{3}$ vs; $p=$ 0.52, Paired T-test). Consistent with these results, there was no evidence of increased cell death in the SG of Atoh1 $1^{\text {cre }}$ Pou $4 \mathrm{fl}^{\text {flox/- }}$ animals based on a TUNEL assay at P1 (data not shown). Finally, the innervation of the organ of Corti appeared largely normal with no evidence of the neural overgrowth that was observed in germline Pou4fl mutants (Huang et al., 2001). These results suggest that both the cell death and neuronal guidance phenotypes seen in the germline Pou $4 \mathrm{fl}^{-/-}$mutants were avoided by using ectopic expression of Atoh $1^{\text {cre }}$ to drive deletion of Pou4fl beginning at E14.5. 


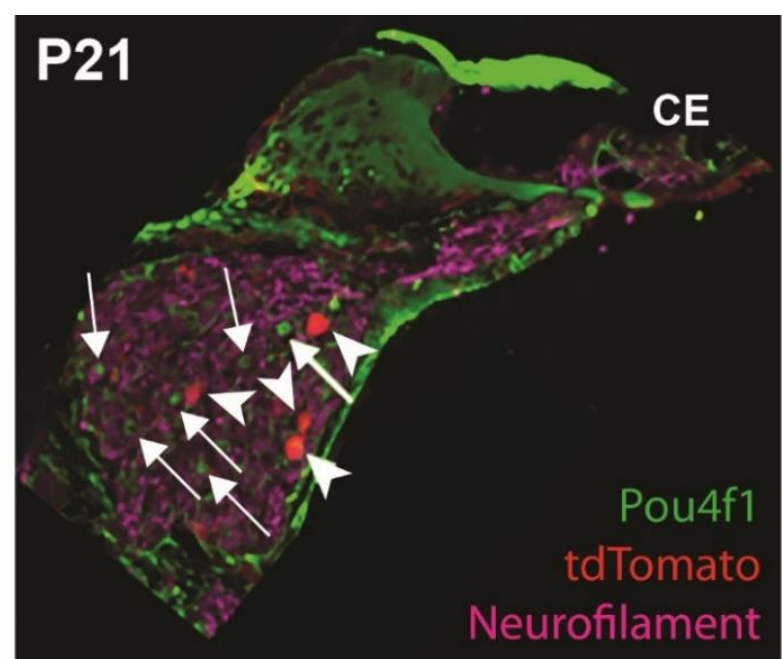

Figure 3. Pou $4 \mathrm{fI}^{\text {creErt2 }}$-mediated recombination occurs in cells that are not positive for Pou $4 \mathrm{f} 1$

Induction of Pou 4 I $^{\text {creErt2 }}$ at P14 labeled SGNs that were not positive for Pou $4 \mathrm{f} 1$ protein after 7 days (P21). Arrows indicate Pou $4 \mathrm{fl}^{+}$SGNS while arrowheads indicate SGNs that express tdTomato as a result of recombination. There is limited overlap between the two sets of SGNs. Scale bar $=50 \mu \mathrm{m}$.
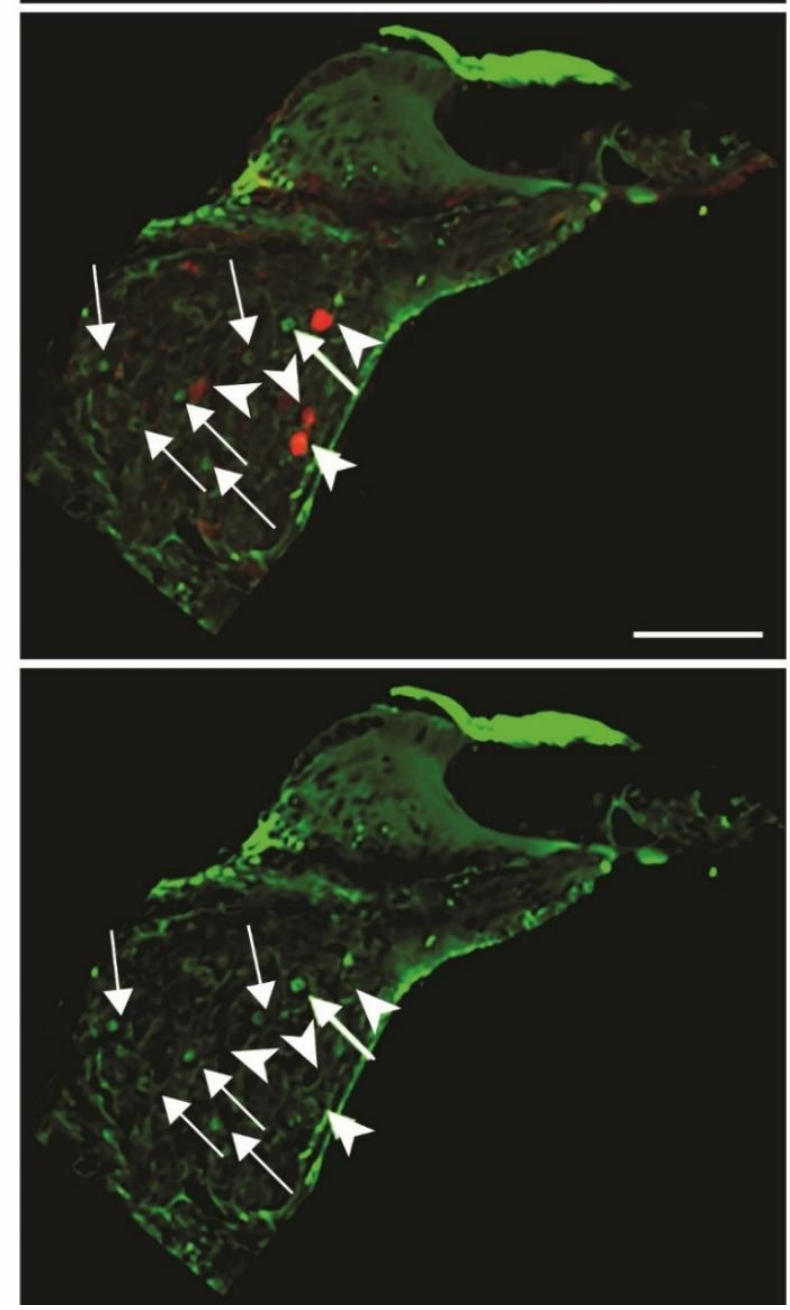


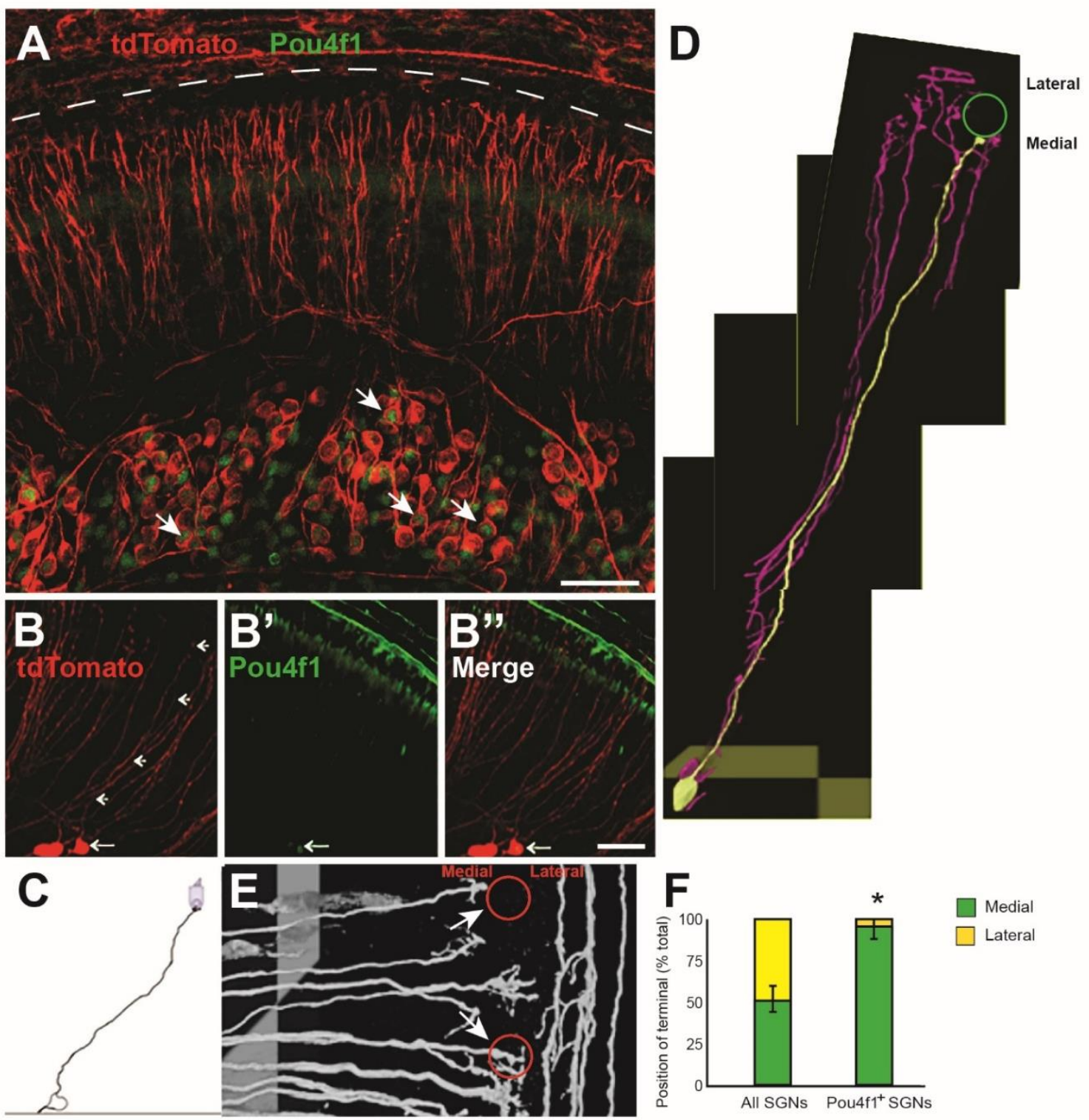

\section{Figure 4. Pou4f1+ SGNs terminate on the modiolar sides of IHCs}

(A) Image of the basal turn from a sparsely-labeled $\mathrm{NgnI}^{\text {creErt2 }} ; \mathrm{R}^{26 R^{\text {tdtomato }}}$ at P7. A subset of the labeled SGN cells (red) are positive for Pou4f1 (green, arrows). (B-B') Images from another example of SGN sparse-labeling. The large arrow at the bottom of the images indicates an SGN-cell body that is positive for Pou4f1 (green). Small arrows in B trace the peripheral axon from that labeled cell body. (C) line drawing for the labeled SGN in B. (D) Composite 3-dimensional reconstruction of a single Pou4f1+ SGN (yellow) which terminates on the modiolar side of an IHC (indicated by green circle). (E) 3dimensional reconstruction of sparsely-labeled SGN terminals. IHC positions indicated by red circles. Fibers that terminate on the modiolar (upper arrow) or the pillar (lower arrow) side of IHCs can be identified. (F) Quantification of terminal locations for all sparsely labeled SGN fibers and for Pou 4f1+ fibers. Slightly more than half $(51 \% ; 95 \% \mathrm{CI}=43 \%-59 \%, \mathrm{n}=154$ terminals) of all labeled fibers terminate on the modiolar side of IHCs. In contrast $96 \%\left(95 \% \mathrm{CI}=88 \%-100 \%, \mathrm{n}=24\right.$ cells, $\mathrm{p}=1.43 \times 10^{-}$ ${ }^{6}$, binomial test) of Pou4f1 $1^{+}$fibers terminate on the modiolar side of IHCs. Scale bar in A, B" $=50 \mu \mathrm{m}$ 


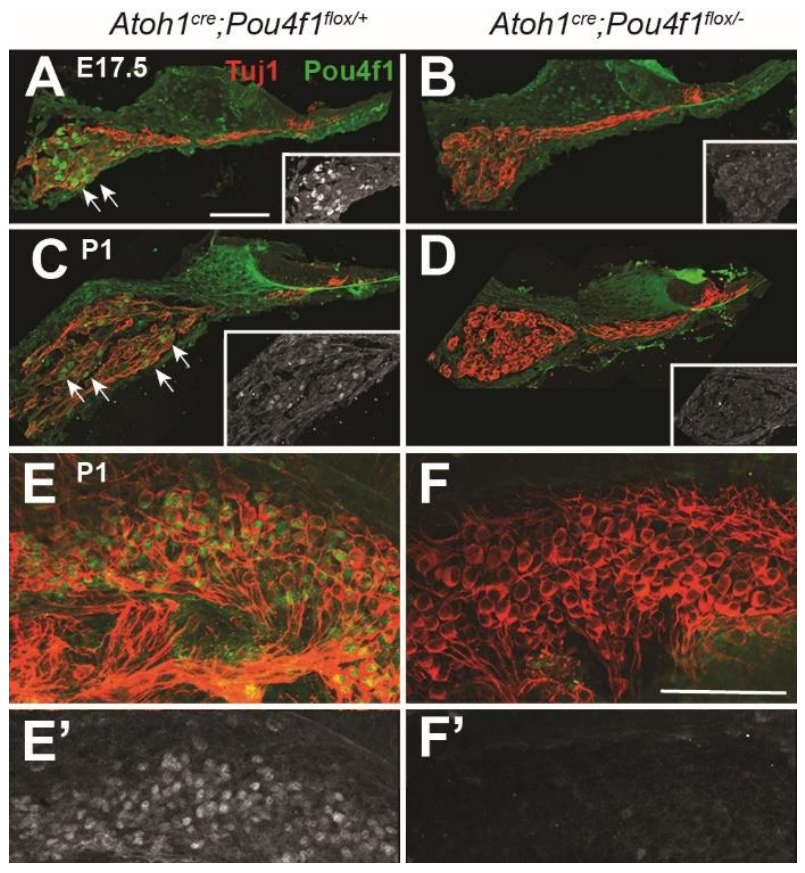

Figure 5. Ectopic Atoh1 ${ }^{\text {cre }}$ expression in SGN successfully deletes Pou4f1 by E17.5

(A-D) Mid-modiolar cross-sections through the apical turns of cochleae at the indicated ages. SGNs are labeled with Tuj1. In control (AtohI ${ }^{\text {cre }} ;$ Pou $4 f f^{f /+}$ ) multiple SGNs are positive for Pou4f1 at both E17.5 and P1 (arrows). In contrast, in cochleae from Atohl ${ }^{\text {cre }} ;$ Pou $4 f f^{\text {flox/ }}$ mice only weak (at E17.5) or no (at P1) expression of Pou4f1 is present in the SGN. Insets show Pou4f1 expression in the SGN in gray scale. (E, F) Whole mount views of the SG from the middle region of the cochlea at P1 labeled as in A. Many SGN nuclei express Pou4f1 in the control, but no Pou4f1 expression is observed in the Atoh $1^{\text {cre }} ;{\text { Pou } 4 f 1^{\text {flox/ }} \text {. }}_{\text {. }}$ E, F) Pou4f1 expression in gray scale. Scale bar in A (same in B-D) $=50 \mu \mathrm{m}$. Scale bar in in F (same in E) $=100 \mu \mathrm{m}$.

\section{Normal pattern of afferent innervation the IHC Atoh $1^{\text {cre }}$;Pou $4 \mathrm{f1}^{\text {flox/- }}$ SGNs $x$}

To determine whether deletion of Pouffl caused a change in the distribution or morphology of SGN synapses, the position and immunofluorescence intensity (proxy of "ribbon size", Wong et al., 2014, Ohn et al., 2016) of the synaptic ribbons at P14 were analyzed and mapped onto the surfaces of IHCs as previously described (Figure 6A,B, see Ohn et al., 2016). A confocal mapping of synaptic locations using immunostainings against CtBP2 indicated no changes in the

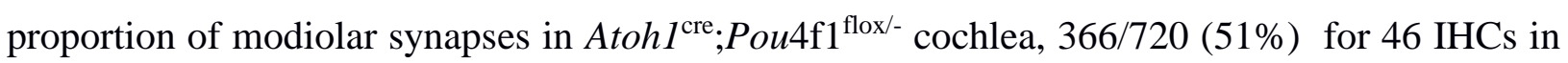
Atoh $1^{\text {cre. }}$ Pou $4 \mathrm{f}^{\text {flox/- }}$ mice vs. 350/726 (48\%) synapses for 48 IHCs in control mice. Interestingly, in contrast to our previous report in P14-18 C57bl6/J mice (Ohn et al., 2016), CtBP2 immunofluorescence intensity, on average, was greater for pillar synapses (Figure $6 \mathrm{C}$ ). This difference could be related to different strains (mix of C57bl6/J and CD1 in this study) and developmental stage analyzed as a similar spatial gradient to the one observed here was reported for CBA mice in the third postnatal week as well (Liberman and Liberman, 2016). 
A

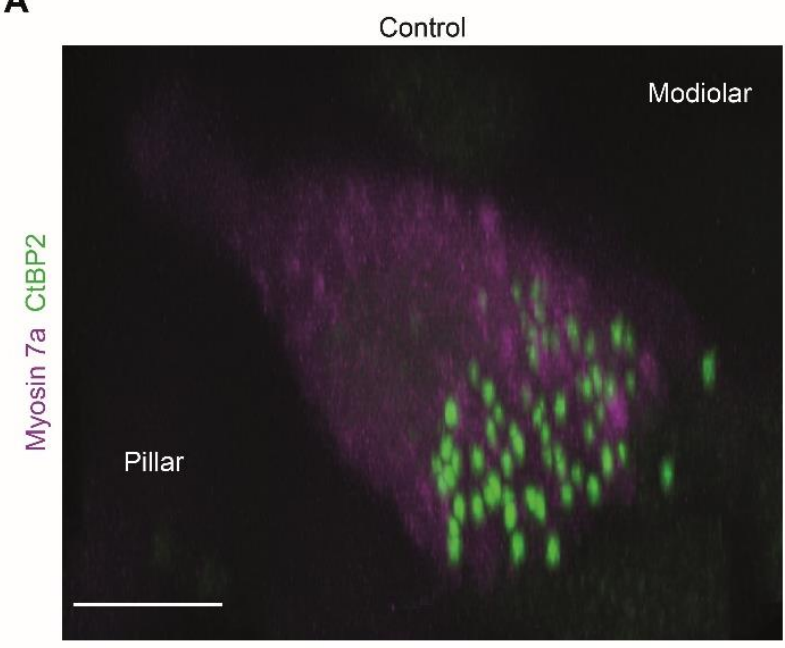

B

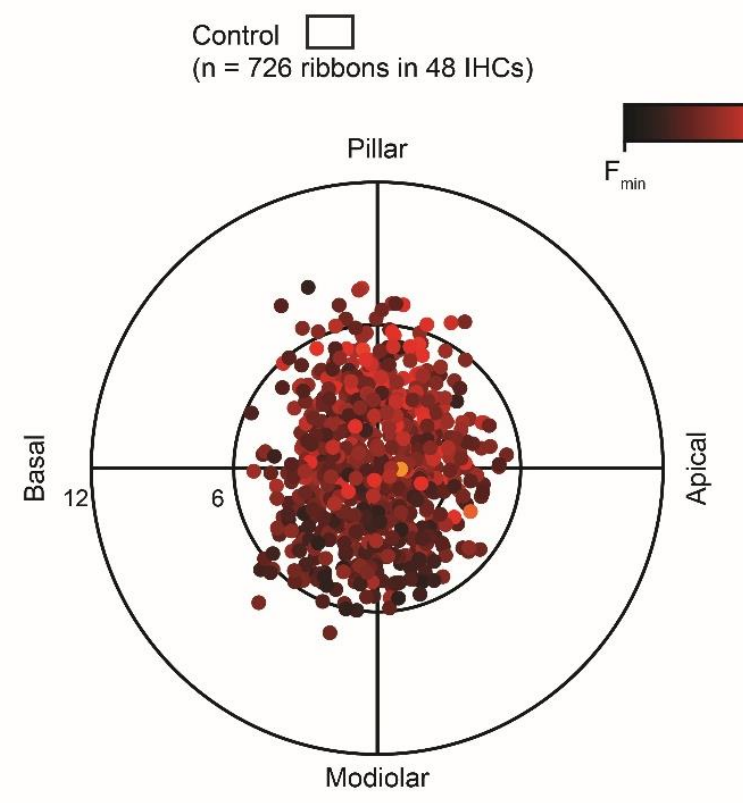

C

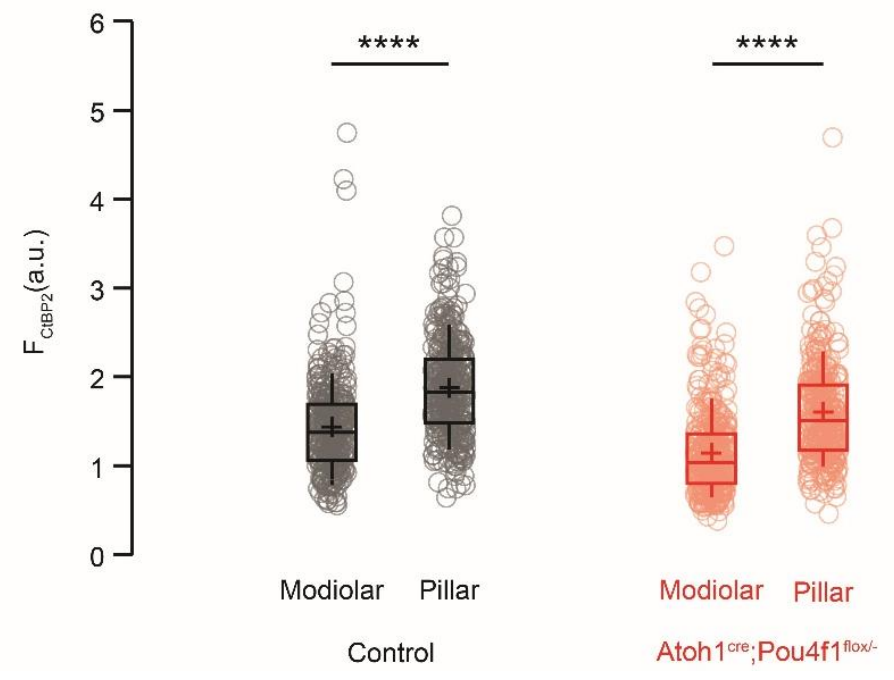

$\mathrm{F}_{\mathrm{CABP} 2}$

Atoh $1{ }^{\text {cre }}$ :Pou $4 f_{1} 1^{\text {flox- }}$ -

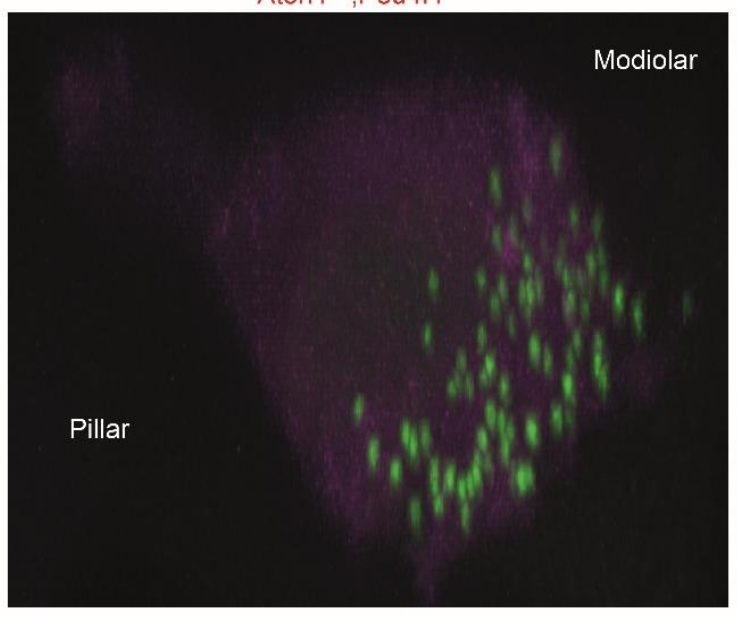

Atoh1 $1^{\text {cre }} ;$ Pou $4 f^{1 \text { flox- }}$ -

( $n=720$ ribbons in $46 \mathrm{IHCs}$ )

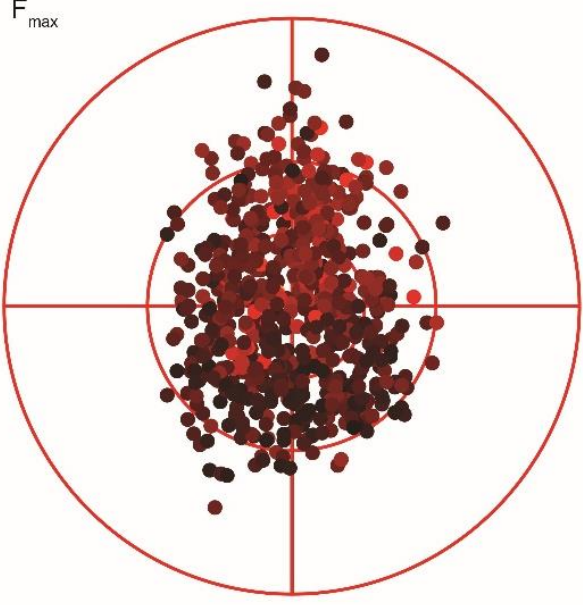


Figure 6. IHC synapse numbers and distributions are unchanged in Atoh1 ${ }^{\text {cre }}$ Pou 4 fl $^{\text {flox/- }}$ cochleae

(A) Maximal projection (side view) of a row of IHCs labelled in magenta against Myosin 7a and their synaptic ribbons labelled in green against CtBP2 from control and Atoh $1^{\text {cre }}$;Pou $4 f^{f f l o x /}$ cochleae at P14.(B) Polar charts (in $\mu \mathrm{m}$ ) display locations and intensities of CtBP2-labelled synaptic ribbons. Orientations are indicated. (C) By comparison with control, synapses in $A t o h 1^{\text {cre }}$;Pou $4 \mathrm{fl}^{\text {flox/- }}$ cochlea show decreased overall CtBP2 labeling intensity $(n=726$ ribbons from 48 IHCs in control and 720 ribbons from 46 IHCs in AtohI ${ }^{\text {cre }} ;$ Pou $4 f 1^{\text {flox/- }} ; p<0.0001$, Mann-Whitney-Wilcoxon test). For both Control and Atohl ${ }^{\text {cre }} ;$ Pou $4 f 1^{\text {flox/- }}$ IHCs a significant gradient of ribbon strength was observed along the pillar-modiolar axis (Control: Modiolar: $n=360$ spots; Pillar: $n=376$ spots; Atoh1 $1^{\text {cre }}$; Pou4f1 ${ }^{\text {flox-- }}$ : Modiolar: $n=366$ spots; Pillar: $n=$ 354 spots, $p<0.0001$, Mann-Whitney-Wilcoxon test for both conditions). Box plots show 10, 25, 50, 75 and 90th percentiles with individual data points overlaid, means are shown as crosses.

\section{Atoh1 ${ }^{\text {cre }}$;Pou $4 \mathrm{f1}^{\text {flox/- }}$ mice exhibit an increased voltage-sensitivity of activation of the $\mathrm{Ca}^{2+}$ influx at the whole-cell and single synapse levels at IHCs}

Next, since the immunofluorescence intensity but not the position of the presynaptic ribbons was affected by the lack of Pou4fI expressing type I SGNs, we tested for an instructive trans-synaptic influence of this subpopulation on the presynaptic $\mathrm{Ca}^{2+}$-influx at IHCs. We performed whole-cell patch-clamp recordings of presynaptic voltage-gated $\mathrm{Ca}^{2+}$-influx in IHCs (more than 90\% mediated by the Cav1.3 $\mathrm{Ca}^{2+}$-channels (Brandt et al., 2003; Dou et al., 2004; Platzer et al., 2000))

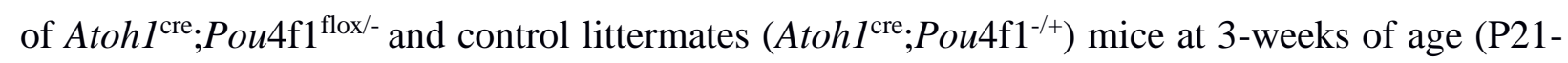
28). Using voltage step-depolarizations in conditions isolating the $\mathrm{Ca}^{2+}$-influx (see methods); we probed its amplitude and voltage-dependence (Figure 7A). The amplitude of $\mathrm{Ca}^{2+}$-influx (Figure $7 \mathrm{~A}_{\mathrm{i}}$ ) was unaltered in Atoh $1^{\text {cre }} ;$ Pou $4 \mathrm{f} 1^{\text {flox/- }}$ mice $(-173 \pm 6$ pA, S.D. $=38$ pA, $n=44$ IHCs, $n=14$ in Atohl $1^{\text {cre }}$;Pou $4 \mathrm{f} 1^{\text {flox/- }}$ vs. $-161 \pm 7$ pA, S.D. $=49$ pA, $n=44 \mathrm{IHCs}, n=16$ in Atoh ${ }^{\text {cre }} ;$ Pou $4 \mathrm{fl}^{-/+}$; $p=0.20$, Student T-test). We then analyzed the voltage-dependence of $\mathrm{Ca}^{2+}$-channel activation (Figure 7B), and found a small but significant hyperpolarizing shift of the voltage potential of half-maximal $\mathrm{Ca}^{2+}$-channel activation, $\mathrm{V}_{\mathrm{h}}$ (Figure $7 \mathrm{~B}_{\mathrm{i}}$; $-24.95 \pm 0.47 \mathrm{mV}$, S.D. $=3.11 \mathrm{mV}, n=$ $44 \mathrm{IHCs}, n=14$ in Atoh ${ }^{\text {cre }}$;Pou4f1 $1^{\text {flox/- }}$ mice vs. $-23.30 \pm 0.60 \mathrm{mV}$, S.D. $=3.96 \mathrm{mV}, n=44 \mathrm{IHCs}$, $n=16$ in Atoh $1^{\text {cre }}$ Pou $4 \mathrm{f1}^{-/+}$mice; $p=0.032$, Student T-test). This shift was primarily caused by an increased voltage-sensitivity of $\mathrm{Ca}^{2+}$-channel activation (reflected by a decrease of the slope factor k) in Atoh $1^{\text {cre }}$;Pou $4 \mathrm{f} 1^{\text {flox } /-}$ IHCs $(7.63 \pm 0.07 \mathrm{mV}$, S.D. $=0.45 \mathrm{mV}, n=44 \mathrm{IHCs}, n=14$ in the experimental vs. $7.97 \pm 0.10 \mathrm{mV}$, S.D. $=0.65 \mathrm{mV}, n=44 \mathrm{IHCs}, n=16$ in the control, $p=$ 0.017, Mann-Whitney-Wilcoxon test, Figure 7Bii). 
To record the $\mathrm{Ca}^{2+}$-influx at single presynaptic AZs (Frank et al., 2009) we loaded the IHCs with a low-affinity $\mathrm{Ca}^{2+}$-indicator dye $(800 \mu \mathrm{M}$ Fluo-4FF) and used a spinning-disk confocal microscope that allows fast registering and recording of the majority of the IHC synapses (Ohn et al., 2016). We chose experimental conditions (addition of $10 \mathrm{mM}$ of the synthetic $\mathrm{Ca}^{2+}$ chelators EGTA) in which the $\mathrm{Ca}^{2+}$-indicator fluorescence is a proxy for the $\mathrm{Ca}^{2+}$-influx (Frank et al., 2009; Ohn et al., 2016; Jean et al., 2018). Prior to analysis of $\mathrm{Ca}^{2+}$-influx, we imaged fluorescentlyconjugated CtBP2-binding peptide (Zenisek et al., 2004), which bound to the ribbons occupying the AZs. We then employed ramp-depolarizations to assess amplitude and voltage-dependence of $\mathrm{Ca}^{2+}$-influx from the bottom-most to top-most ribbon (from basal to nuclear level of the IHC; see methods). For simplicity, we refer to "synaptic $\mathrm{Ca}^{2+}$-influx" when describing observations based on hotspots of $\mathrm{Ca}^{2+}$-indicator fluorescence co-localizing with a marked ribbon at the basolateral IHC membrane. We found comparable maximal amplitudes of the background-normalized maximal fluorescence increase between AZs of control and Atohl $1^{\text {cre }} ; P o u 4 f 1^{\text {flox } /-}$ IHCs $\left(\Delta \mathrm{F} / \mathrm{F}_{\max }\right.$; $0.86 \pm 0.03$, S.D. $=0.44, n=203$ AZs in $20 \mathrm{IHCs}, n=10$ in Atoh $1^{\text {cre }} ;$ Pou $4 f 1^{\text {flox } /-}$ vs. $0.88 \pm 0.03$, S.D. $=0.45, n=155 \mathrm{AZs}$ in $17 \mathrm{IHCs}, n=9$ in controls, $p=0.46$, Mann-Whitney-Wilcoxon test; Figure $7 \mathrm{C}_{\mathrm{i}}$ ). This indicates an unchanged number of synaptic $\mathrm{Ca}^{2+}$-channels at IHC AZs in Atoh $1^{\text {cre }}$;Pou $4 \mathrm{f}^{\text {flox/- }}$ animals and agrees with the observation of unchanged whole-cell $\mathrm{Ca}^{2+}-$ current amplitudes.

Next, we analyzed the voltage-dependence of activation for the synaptic $\mathrm{Ca}^{2+}$-influx as previously described (Ohn et al., 2016; Jean et al., 2018). Analysis of fractional activation revealed a slight tendency for the $\mathrm{V}_{\mathrm{h}}$ to be shifted towards more hyperpolarized potentials in Atoh $1^{\text {cre }} ;$ Pou $4 \mathrm{f}^{\text {flox/- }}$ IHCs that did not reach significance $(-25.54 \pm 0.45 \mathrm{mV}$, S.D. $=5.80 \mathrm{mV}, n$ $=168 \mathrm{AZs}$ in $20 \mathrm{IHCs}, n=10$ in the Atoh $1^{\mathrm{cre}} ;$ Pou $4 \mathrm{fl}^{\mathrm{flox} / \mathrm{-}}$ vs. $-24.11 \pm 0.61 \mathrm{mV}$, S.D. $=7.20 \mathrm{mV}$, $n=139 \mathrm{AZs}$ in $17 \mathrm{IHCs}, n=9$ in the control, $p=0.13$, Mann-Whitney-Wilcoxon test; Figure $7 \mathrm{D}_{\mathrm{i}}$ ). As for the whole-cell $\mathrm{Ca}^{2+}$-influx, the voltage sensitivity was significantly increased on the single AZ level $(6.66 \pm 0.12 \mathrm{mV}$, S.D. $=1.58 \mathrm{mV}, n=168 \mathrm{AZs}$ in $20 \mathrm{IHCs}, n=10$ in the Atohl $1^{\text {cre }} ;$ Pou $4 \mathrm{f} 1^{\text {flox/- }}$ vs. $7.05 \pm 0.16 \mathrm{mV}$, S.D. $=1.92 \mathrm{mV}, n=139$ AZs in 17 IHCs, $n=9$ in the control, $p=0.045$, Mann-Whitney-Wilcoxon test; Figure $\left.7 \mathrm{D}_{\mathrm{ii}}\right)$. 


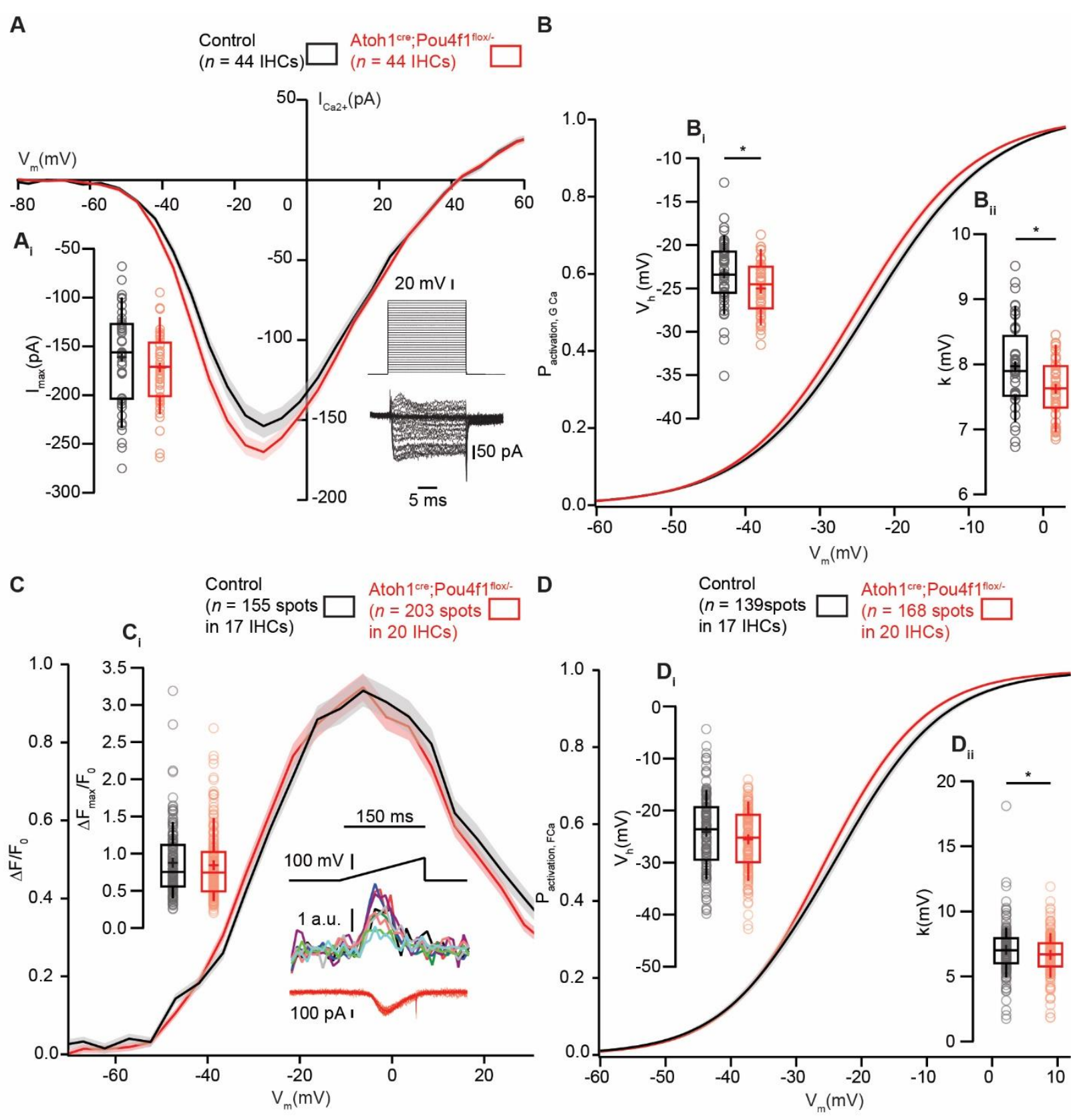

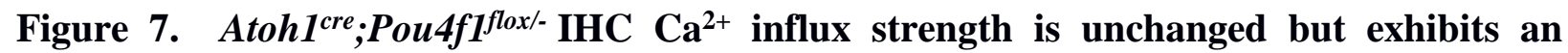
increased voltage sensitivity

(A) IV-relationship of the whole-cell $\mathrm{Ca}^{2+}$-current in Control and Atohl ${ }^{\text {cre }} ; P$ Pou $4 \mathrm{ff}^{\text {flox/- }}$ IHCs showed comparable current amplitudes ( $n=44 \mathrm{IHCs}, n=14$ in the Atoh $1^{\text {cre }} ; P$ ou $4 \mathrm{fl} 1^{\text {flox } /-} ; n=44 \mathrm{IHCs}, n=16$ in the $\mathrm{WT} ; p=0.20$, t.test, $\mathbf{A}_{\mathbf{i}}$. The protocol, consisting of $20 \mathrm{~ms}$ steps of $5 \mathrm{mV}$ from -82 to $+63 \mathrm{mV}$, as well as the resulting currents, are shown in the bottom right. Mean (line) \pm S.E.M. (shaded areas) are displayed, the box plots show 10, 25, 50, 75 and 90th percentiles with individual data points overlaid and means are shown as crosses, as for (B, C, and D). (B) Fractional activation of the whole-cell $\mathrm{Ca}^{2+}$-current derived from the IV-relationships (A) was fitted to a Boltzmann function. $\mathbf{B}_{\mathbf{i}}$. Box plots of the voltage for halfmaximal activation $\mathrm{V}_{\mathrm{h}}$ and $\mathrm{V}_{\mathrm{h}}$-estimates of individual IHCs show a hyperpolarized shift of the fractional activation of the Cav1.3 $\mathrm{Ca}^{2+}$-channels in Atohl ${ }^{\text {cre }}$;Pou $4 f^{f l o x /}$ condition ( $\mathrm{n}=44 \mathrm{IHCs}, \quad n=14$ in the Atoh $1^{\text {cre }} ;$ Pou $4 \mathrm{fl}^{\text {flox-- }} ; n=44 \mathrm{IHCs}, n=16$ in the Control; $p=0.032$, t-test). B $_{\mathrm{ii}}$. Box plots of the voltagesensitivity or slope factor $\mathrm{k}$ and $\mathrm{k}$-estimates of individual IHCs illustrate increased voltage sensitivity in 
the mutant condition ( $\mathrm{n}=44 \mathrm{IHCs}, n=14$ in the Atoh $1^{\text {cre }}$; Pou $4 \mathrm{f} 1^{\text {flox }--;} n=44 \mathrm{IHCs}, n=16$ in the Control; $p=0.017$, Mann-Whitney-Wilcoxon test). (C) Voltage-ramps from -87 to $+63 \mathrm{mV}$ during $150 \mathrm{~ms}$ (C: right top) were used to trigger synaptic hotspots of Fluo-4FF fluorescence (C: right middle, $10 \mathrm{AZs}$ in one exemplary IHC), and IHC Ca ${ }^{2+}$-influx (middle bottom). FV-relationship $\left(\Delta \mathrm{F} / \mathrm{F}_{0}\right.$ vs. depolarization level in ramp): approximating the voltage-dependence of synaptic $\mathrm{Ca}^{2+}$-influx. $\mathbf{C}_{\mathbf{i}} \cdot \Delta \mathrm{Fmax} / \mathrm{F}_{0}$ was calculated by averaging 5 values at the FV-peak and was comparable between Control and Atoh ${ }^{\text {cre }} ;$ Pou $4 \mathrm{f} 1^{\text {flox/- }}$ conditions ( $\mathrm{n}=203 \mathrm{AZs}$ in $20 \mathrm{IHCs}, n=10$ in the Atoh $1^{\text {cre }} ;$ Pou $4 \mathrm{f} 1^{\text {flox/-}} ; n=155$ AZs in $17 \mathrm{IHCs}, n=9$ in the WT; $p=0.46$, Mann-Whitney-Wilcoxon test). (D) Fractional activation curves derived from fits to the FV-relationships (C) were fitted to a Boltzmann function. $\mathbf{D}_{\mathbf{i}}$. The voltage for half-maximal activation $\mathrm{V}_{\mathrm{h}}$ was not significantly different between Control and Atoh $1^{\text {cre }} ; P$ Pou $4 \mathrm{f} 1^{\text {flox/- }}$ conditions $(\mathrm{n}=168 \mathrm{AZs}$ in 20 IHCs, $n=10$ in the Atoh $1^{\text {cre }}$;Pou4f $1^{\text {flox }-} ; n=139$ AZs in 17 IHCs, $n=9$ in the Control; $p=0.13$, MannWhitney-Wilcoxon test), while the voltage-sensitivity $\mathbf{D}_{\mathbf{i i}}$ was increased in Atohl $^{\text {cre }} ;$ Pou $_{\text {f }} 1^{\text {flox/- }}$ ( $\mathrm{n}=168$ AZs in $20 \mathrm{IHCs}, n=10$ in the Atoh $1^{\text {cre }} ;$ Pou $4 \mathrm{f} 1^{\text {flox } /-;} n=139$ AZs in $17 \mathrm{IHCs}, n=9$ in the Control; $p=0.045$, Mann-Whitney-Wilcoxon test).

\section{Changes in the position-dependence of $\mathrm{AZ}$ properties in $\operatorname{Atoh}^{\text {cre }} ; P$ Pou $4 \mathrm{f1}^{\text {flox/- }}$ IHCs}

Since Pou4f1 is expressed only in a subset of modiolar targeting SGNs, likely presenting low spontaneous rate, high threshold SGNs, we assess whether the changes in presynaptic $\mathrm{Ca}^{2+}$-influx, observed upon postsynaptic Pou4f1 disruption, depend on position along the modiolar-pillar axis. In order to study the position-dependent properties of the AZs of multiple IHCs, we reconstructed the IHCs in cylindrical coordinates and identified the positions of their synapses (for more details see methods and Ohn et al., 2016). It has been previously shown that the synapses located on the pillar side present stronger $\mathrm{Ca}^{2+}$ influx (Meyer et al., 2009; Ohn et al., 2016), stronger ribbons and more depolarized activation of the Cav1.3 channels as compared to synapses on the modiolar side which show opposite properties (Ohn et al., 2016). Consistent with the above immunolabeling result (Figure 6), live imaging of the AZs on the modiolar side presented weaker fluorescence of the CtBP2-binding peptide than the pillar side AZs in both genotypes (Atoh $1^{\text {cre }}$;Pou4f $1^{\text {flox/- }}$ : modiolar: $2.67 \pm 0.09$, S.D. $=1.02, n=137$ AZs vs pillar: $3.26 \pm 0.14$, S.D. $=1.22, n=76$ AZs, $n$ $=10 ; p=0.00007$; Control: modiolar: $2.28 \pm 0.07$., S.D.=0.72, $n=119$ AZs vs. pillar: $2.78 \pm$ 0.13., S.D. $=0.93, n=52 \mathrm{AZs}$ in $17 \mathrm{IHCs}, n=9 ; p=0.00006$, Mann-Whitney-Wilcoxon test for both conditions). Moreover, we found that, on average, CtBP2-binding peptide labeling of ribbons was significantly stronger in IHCs in Atoh $1^{\text {cre }} ; P o u 4 \mathrm{fl}^{\text {flox/- }}$ by comparison with control (Control: $2.43 \pm 0.06, n=171 \mathrm{AZs}$ ) vs. Atohl ${ }^{\text {cre }} ;$ Pou $4 f 1^{\text {flox/- }}: 2.88 \pm 0.08, n=213$ AZs, $p=$ 0.00002 , Mann-Whitney-Wilcoxon test) (Figure 8A). In both, Atoh $1^{\text {cre }}$ Pou $4 \mathrm{f} 1^{\text {flox/- }}$ and control IHCs (Atoh $1^{\text {cre }}$; Pou $4 f 1^{\text {flox/- }}$ ) the AZs of the modiolar side exhibited a slightly more depolarized activation of $\mathrm{Ca}^{2+}$-channels when compared to the pillar side (Atohl ${ }^{\text {cre. }}$ Pou $4 \mathrm{fl}^{\text {flox/- }}$, modiolar : $24.61 \pm 0.54 \mathrm{mV}$, S.D. $=5.62 \mathrm{mV}, n=108$ AZs vs pillar: $-27.21 \pm 0.74 \mathrm{mV}$, S.D. $=5.78 \mathrm{mV}, n$ 
$=60 \mathrm{AZs}$ in $20 \mathrm{IHCs}, n=10 ; p=0.011$; Control: modiolar: $-23.05 \pm 0.71 \mathrm{mV}$, S.D. $=6.95 \mathrm{mV}$, $n=96$ AZs vs. pillar: $-26.47 \pm 1.12 \mathrm{mV}$, S.D. $=7.32 \mathrm{mV}, n=43 \mathrm{AZs}, n=9 ; p=0.0056$, MannWhitney-Wilcoxon test for both conditions, Figure 8C).

A
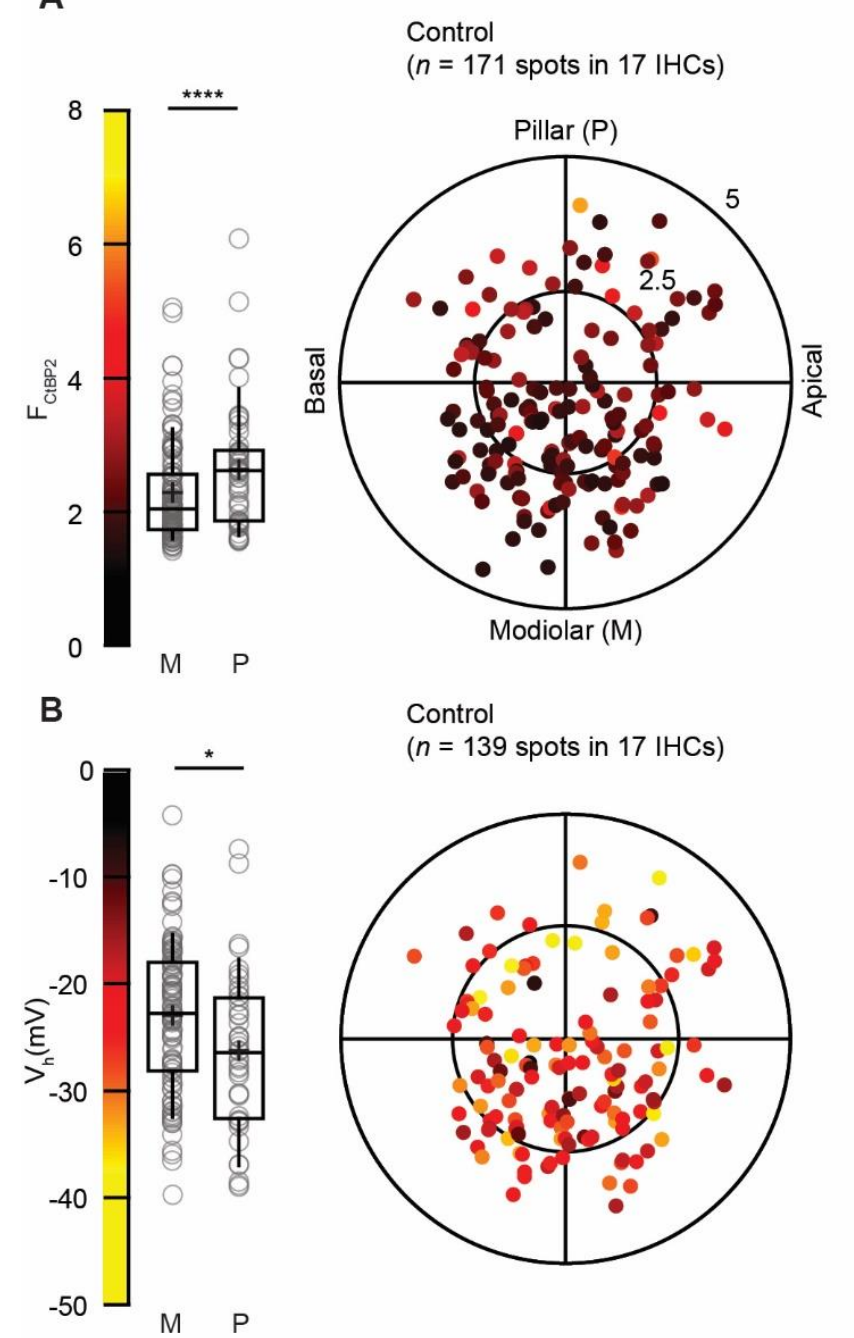

Control

$(n=139$ spots in $17 \mathrm{IHCs})$

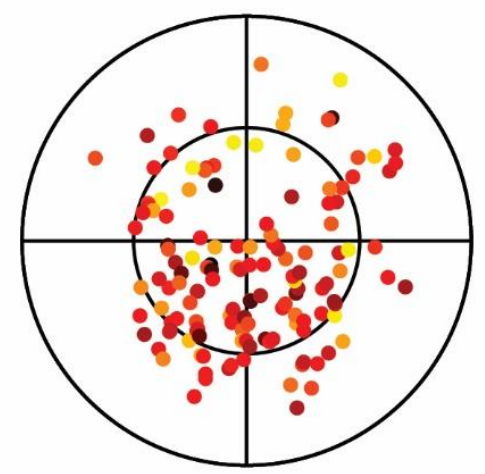

Atoh1 $1^{\text {cre }} ;$ Pou $4 f 1^{\text {flox/- }}$

( $n=213$ spots in $20 \mathrm{lHCs})$

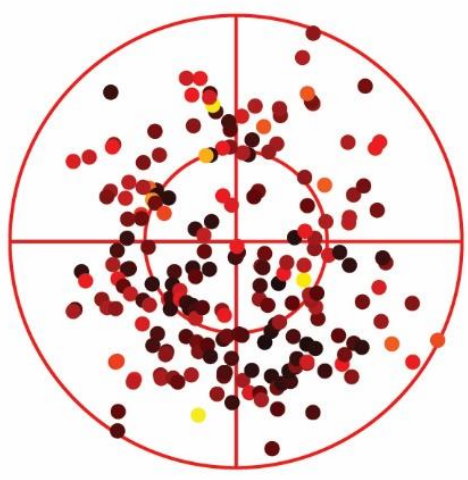

Atoh $1^{\text {cre }} ;$ Pou $41^{\text {floxt- }}$

( $n=168$ spots in $20 \mathrm{lHCs}$ )

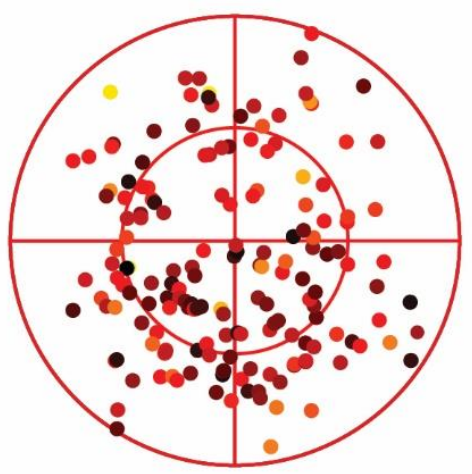

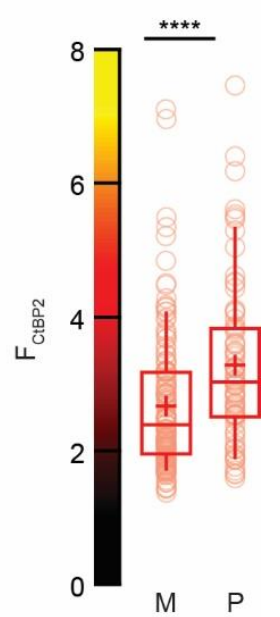

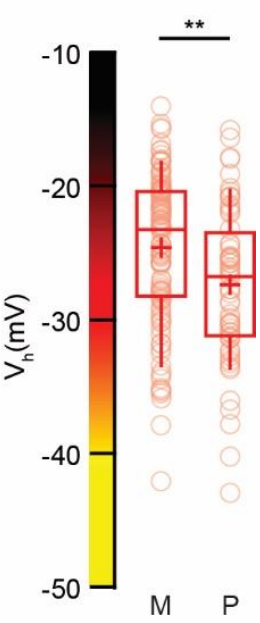

Figure 8. Spatial gradients for ribbon size and voltage dependence of synaptic $\mathrm{Ca}^{2+}$ influx are preserved in Atoh $^{\text {cre }}$;Pou 4 fi $^{\text {flox/- IHCs. }}$ IHC

(A) Polar charts (in $\mu \mathrm{m}$ ) display locations and intensities of AZ TAMRA-peptide (targeting the ribbons) fluorescence in live-imaging experiments. Box plots of the ribbon fluorescence $\mathrm{F}_{\mathrm{CtBP} 2}$ and $\mathrm{F}_{\mathrm{CtBP} 2}$-estimates of individual IHCs indicate that both Control and AtohI ${ }^{\text {cre }}$;Pou $4 f^{f l o x /}$ IHCs show significantly stronger ribbons on the pillar side as compared to the modiolar (Control: modiolar: $n=119 \mathrm{AZs}$; pillar: $n=52 \mathrm{AZs}$, $p=0.00006$; Atoh1 $1^{\text {cre }}$ Pou4f1 ${ }^{\text {floxl- }}$ : modiolar: $n=137$ AZs vs Pillar: $n=76$ AZs, $p=0.00007$; MannWhitney-Wilcoxon test for both conditions). (B) Polar charts display voltages for half-maximal activation $\mathrm{V}_{\mathrm{h}}$ as a function of $\mathrm{AZ}$ positions in live-imaging experiments. Box plots of the voltage for half-maximal activation $\mathrm{V}_{\mathrm{h}}$ and $\mathrm{V}_{\mathrm{h}}$-estimates of individual IHCs show a significant hyperpolarized shift of the fractional activation of the Cav1.3 channels in the pillar side compared to modiolar in both Control and AtohI ${ }^{\text {cre }}$;Pou4ff flox/ $^{\text {IHCs }}$ (Control: modiolar: $n=96$ AZs; pillar: $n=43$ AZs, $p=0.011$; Atoh $1^{\text {cre }}$ Pou4f1 ${ }^{\text {flox/- }}$ : modiolar: $n=108 \mathrm{AZs}$; pillar: $n=60 \mathrm{AZs}, p=0.0056$; Mann-Whitney-Wilcoxon test 
for both conditions). Data were pooled from 17 and 20 IHCs in Control and Atohl ${ }^{\text {cre }}$;Pou4fI ${ }^{\text {flox/ }}$ condition respectively, box plots show $10,25,50,75$ and 90th percentiles with individual data points overlaid; means are shown as crosses.

In agreement with our previous studies (Meyer et al., 2009; Ohn et al., 2016), we found that the AZs on the modiolar side show a stronger $\Delta \mathrm{F} / \mathrm{F}_{0}\left(\Delta \mathrm{F}_{\max } / \mathrm{F}_{0}\right.$, mean of 5 values at the peak $)$ than the ones on the pillar side in the control condition (modiolar: $0.92 \pm 0.05$, S.D. $=0.47, n=$ 106 AZs vs. pillar: $0.79 \pm 0.06$, S.D. $=0.39, n=49$ AZs in 17 IHCs $, n=9, p=0.049$, MannWhitney-Wilcoxon test; Fig 9A, B). In contrast, there was no significant difference in $\Delta \mathrm{F}_{\max } / \mathrm{F}_{0}$ along the modiolar-pillar axis in the Atohl $1^{\text {cre }}$;Pou4f $1^{\text {flox/- }}$ IHCs (modiolar: $0.88 \pm 0.04 .$, S.D. $=$ 0.46, $n=130$ AZs vs. pillar: $0.80 \pm 0.07$, S.D. $=0.40, n=73$ AZs in $20 \mathrm{IHCs}, n=10, p=0.38$, Mann-Whitney-Wilcoxon test; Figure 9A,B).

The $\mathrm{AZ}$ with the strongest $\Delta \mathrm{F}_{\max } / \mathrm{F}_{0}$ for a given cell, nicknamed "winner" (highlighted in blue in Figure 9A), strongly contributes to the observed difference in $\Delta \mathrm{F}_{\max } / \mathrm{F}_{0}$ between modiolar and pillar AZs in control IHCs. The vast majority of winner synapses were located on the modiolar side for both control and Atoh $1^{\text {cre }} ;$ Pou $4 \mathrm{f} 1^{\text {flox/- }}$ animals (15 IHCs/17 IHCs for the control, 17 IHCs $/ 20$ IHCs for the Atoh $1^{\text {cre }} ;$ Pou $4 \mathrm{fl}^{\text {flox } /-}$ ). The ratio mean $\Delta \mathrm{F}_{\text {max }} / \mathrm{F}_{0}$ of winner $/$ mean $\Delta \mathrm{F}_{\max } / \mathrm{F}_{0}$ of the rest of the AZs (for a given cell) tended to be more pronounced in Atoh $1^{\text {cre }} ;$ Pou $4 \mathrm{fl} 1^{\text {flox/- }}$ by comparison with control $\left(2.07 \pm 0.13\right.$ in Atoh $1^{\text {cre }} ;$ Pou $4 \mathrm{f} 1^{\text {flox/- }}$ vs. $1.79 \pm 0.14$ in the control, $p=$ 0.07, Student T-test). Interestingly, when we compared $\Delta \mathrm{F}_{\max } / \mathrm{F}_{0}$ of modiolar and pillar AZs without the winners, there was still a tendency for stronger $\Delta \mathrm{F}_{\max } / \mathrm{F}_{0}$ of $\mathrm{AZs}$ at the modiolar side of control IHCs (modiolar: $0.83 \pm 0.03, n=91$ AZs vs. pillar: $0.78 \pm 0.05,47$ AZs in 17 IHCs, $\boldsymbol{p}$ $=0.20$ ) whereas no such trend remained in Atoh $1^{\text {cre }} ; P o u 4 \mathrm{fl}^{\text {flox/- }}$ animals (modiolar: $0.78 \pm 0.03, n$ $=113 \mathrm{AZs}$ ) vs. pillar: $0.79 \pm 0.05, n=70 \mathrm{AZs}$ in $20 \mathrm{IHCs}, p=0.98$, Figure 9A,B). 
A
Control
( $n=155$ spots in $17 \mathrm{IHCs})$
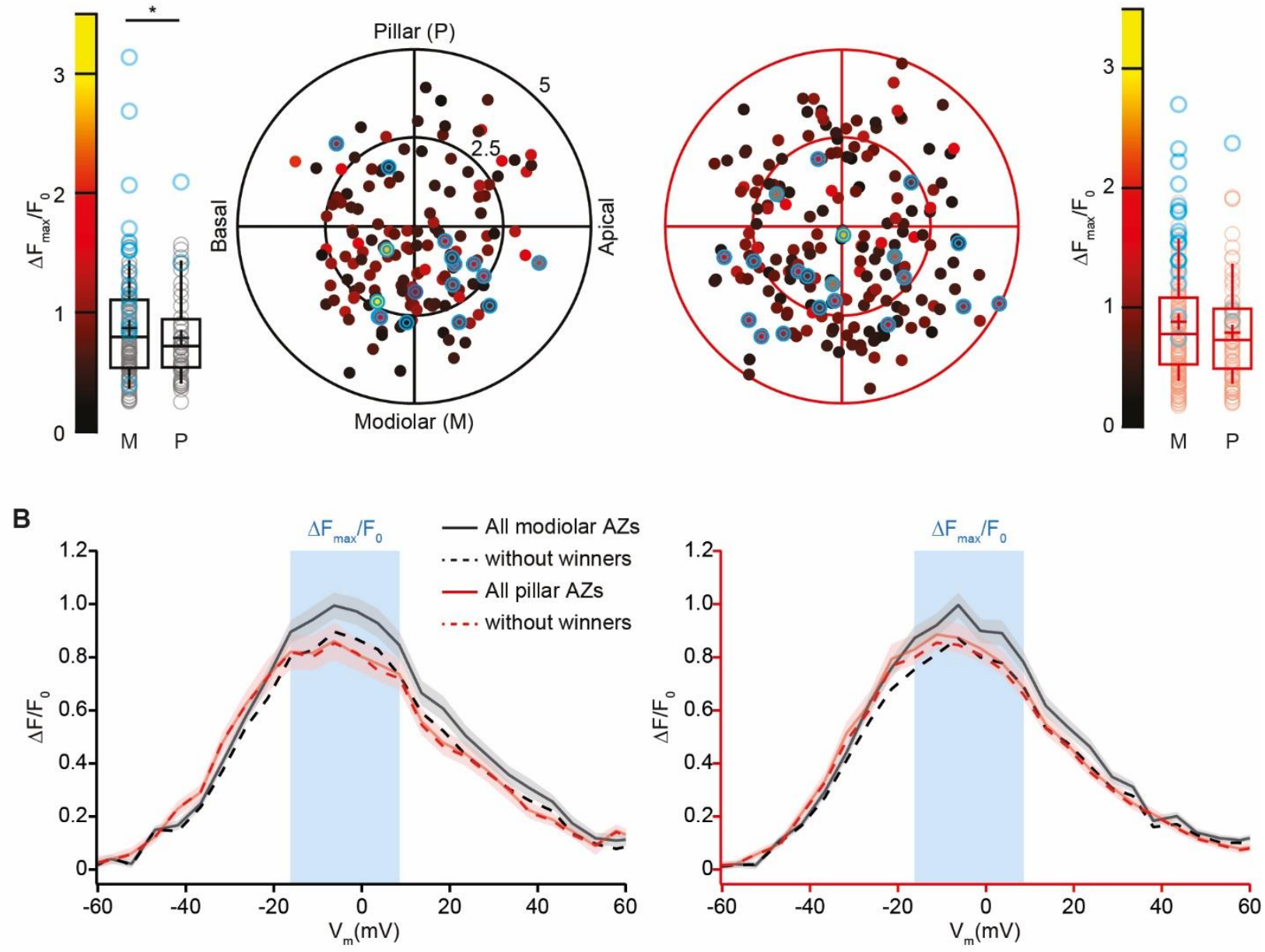

Figure 9. Spatial gradient for synaptic $\mathrm{Ca}^{2+}$ influx strength is collapsed in Atoh1 ${ }^{\text {cre }}$;Pou 4 f1 $^{\text {flox/- IHCs. }}$

(A) Polar charts (in $\mu \mathrm{m})$ display intensities of maximal AZ Ca ${ }^{2+}$-influx $\left(\Delta \mathrm{F}_{\max } / \mathrm{F}_{0}\right)$ as a function of AZ positions in live-imaging experiments. Box plots of the $\Delta \mathrm{F}_{\max } / \mathrm{F}_{0}$ and $\Delta \mathrm{F}_{\max } / \mathrm{F}_{0}$-estimates of individual IHCs show that the Control condition display significantly stronger calcium hotspots on the modiolar side as compared to the pillar side (Control: modiolar: $n=106 \mathrm{AZs}$; pillar: $n=49 \mathrm{AZs}, p=0.049$, Mann-WhitneyWilcoxon test), whereas no significant differences in calcium hotspot strength were observed in Atoh $1^{\text {cre }}$;Pou4f1 $1^{\text {flox/ }}$ ( modiolar: $n=130$ AZs; pillar: $n=73 \mathrm{AZs}, p=0.38$, Mann-Whitney-Wilcoxon test). The strongest AZs from each cell "Winners" are highlighted in blue in both polar charts and box plots. (B) $\mathrm{FV}$-relationship $\left(\Delta \mathrm{F} / \mathrm{F}_{0}\right.$ vs. depolarization level in ramp): approximating the voltage-dependence of synaptic $\mathrm{Ca}^{2+}$-influx in control (left) and Atohl ${ }^{\text {cre }}$;Pou $4 \mathrm{fl}^{\text {flox/- }}$ (right). The modiolar and pillar synaptic $\mathrm{Ca}^{2+}$ influx are black and red, respectively, and displayed as dashed lines when taking out the "Winners". Data were pooled from 17 and $20 \mathrm{IHCs}$ in Control and Atohl ${ }^{\text {cre }}$;Pou $4 \mathrm{fl}^{\text {flox/- }}$ condition respectively. Mean (line) \pm S.E.M. (shaded areas) are displayed, box plots show 10, 25, 50, 75 and 90th percentiles with individual data points overlaid; means are shown as crosses. 


\section{Discussion}

In this study, we identified Pou4f1 as a marker for a subtype of Type I SGNs. These findings are consistent with the results of three recent studies which used single cell mRNA profiling to examine cell types in the spiral ganglion of young adult mice. By tracing individual Pou4f1 expressing neurons using a combination of immunolabeling for Pou $4 \mathrm{f} 1$ and sparse reporter labeling using the $N g n 1^{\text {creErt2 }} ; R 26 R^{\text {tdtomato }}$ mouse, we found that these cells overwhelmingly (96\%, $\mathrm{CI}=88 \%-100 \%, n=24$ cells, $p=1.43 \times 10^{-6}$, binomial test) synapse on the modiolar side of the inner hair cell at P3, a result that is consistent with the findings reported in Petitpre et al. (2018) at more mature time points. These results suggest that Pou4f1 expression corresponds with a group of low spontaneous rate fibers. Combining Pou4fl disruption with physiological and morphological analysis of IHC AZs we propose Pou4f1-positive SGNs instruct presynaptic properties.

\section{Deletion of Pou $4 \mathrm{fl}$ causes changes in presynaptic $\mathrm{AZ}$ properties}

Deletion of Pou4f1 using the ectopic Atoh $1^{\text {cre }}$ mouse line, shortly after SGNs become postmitotic leads to two interesting physiological changes at IHCs: first, there was a subtle but significant increase in voltage sensitivity of the $\mathrm{Ca}^{2+}$-channel activation (on both whole-cell and single synapse levels), inducing a small hyperpolarizing shift of the voltage potential of half-maximal $\mathrm{Ca}^{2+}$-channel activation ( $\mathrm{V}_{\mathrm{h}}$, significant on the level of whole-cell $\mathrm{Ca}^{2+}$-influx). Since it was shown in the cat that the modiolar IHC side is associated with low spontaneous rate, high threshold SGNs (Liberman et al., 1982; Merchan-Perez and Liberman, 1996), we speculate that Pou4f1 expression in SGNs might contribute to this phenotype, by its downstream effectors instructing a lower voltage-sensitivity of $\mathrm{Ca}^{2+}$ channel activation at the presynaptic IHC AZ. Clearly, the shift in voltage-sensitivity is subtle and might not fully account for the physiological phenotype of low spontaneous rate SGNs, which might require additional regulators (Ohn et al., 2016; Jean et al., 2018). In these previous studies, shifts in the voltage-dependence of presynaptic $\mathrm{Ca}^{2+}$ influx of only few $\mathrm{mVs}$ went along with changes in the distribution of the spontaneous rate in SGNs, which was not investigated here. However, it is important to consider that Pou4f1 is only expressed in $30 \%$ of all Type I SGNs, and therefore $70 \%$ of the synapses on any given IHC in a Atoh $1^{\text {cre }}$;Pou 4 f $^{\text {flox/- }}$ cochlea may be phenotypically normal. Moreover, the argument relies on the assumption that these SGNs, in mice too, insert on the modiolar side of the IHC. Additionally, while Pou4f1 disruption leads to an overall hyperpolarized $\mathrm{Ca}^{2+}$-channel activation, the spatial 
gradient of the voltage-dependence of $\mathrm{Ca}^{2+}$-channel activation was maintained. Therefore, more work, including recordings from single SGNs in Atoh $^{\text {cre }}$;Pou $4 \mathrm{fl}^{\text {flox/- }}$ mice, remains to be done to comprehend the effects of the mutation and the molecular mechanisms determining SGN physiology.

Interestingly, the modiolar-pillar gradient in maximal synaptic $\mathrm{Ca}^{2+}$-influx ("AZ

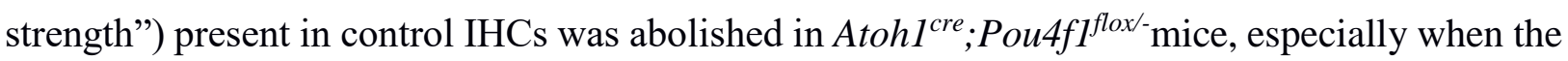
$\mathrm{AZ}$ with the strongest $\mathrm{Ca}^{2+}$-influx (the "Winner") was removed from the analysis. This suggests that Pou4f1 is not important in the formation of the $\mathrm{AZ}$ with the greatest $\mathrm{Ca}^{2+}$ channel complement, the "winner". One interesting possibility is that the winner AZ is the first synaptic SGN contact engaged by an IHC independent of Pou4fl expression and attracts the largest number of $\mathrm{Ca}^{2+}$-channels. However, Pou4f1 seems important in either establishing or maintaining the larger number of $\mathrm{Ca}^{2+}$ channels at the other modiolar AZs as compared to the pillar ones not associated with Pou4f1 expressing SGNs. This instructive influence would set the maximal strength of the presynaptic release and might involve the differential recruitment of presynaptic scaffolds, $\mathrm{Ca}^{2+}$ channel subunits, and proteins interacting with the $\mathrm{Ca}^{2+}$ channels potentially modulating the voltage dependence. Future studies will be required to test this hypothesis. A surprising finding was that of an inverted gradient of our proxies of ribbon size, where the stronger ribbons are found in the pillar side in both control and Atoh1 $1^{\text {cre }} ;$ Pou $4 f 1^{\text {flox/mice }}$ (mix of CD1 and C57bl6/J strains). Both CtBP2 immunofluorescence and Ctbp2-binding peptide fluorescence intensity exhibited stronger signals, indicating larger ribbons, for pillar AZs in IHCs of both control and Atoh $1^{\text {cre }} ; P o u 4 f 1^{\text {flox/- }}$ mice. The finding seems to be robust as it was made by two different methods (immunofluorescence and live-imaging) and at two developmental stages (third and fourth postnatal weeks, respectively). Interestingly, a similar observation was made in the third postnatal week of CBA mice (Liberman and Liberman, 2016) with a gradient for larger synaptic ribbons on the pillar side, in contrast with our previous report at the same ages (Ohn et $a l .$, 2016, C57bl6/J). Nonetheless future studies, ideally involving electron microscopy, should follow-up this observation over several developmental stages (Liberman and Liberman, 2016) and address the issue also in comparison to previous studies on mouse cochleae from different mouse strains (Liberman et al., 2011; Liberman and Liberman, 2016; Ohn et al., 2016).

\section{Potential roles of Pou $4 \mathrm{fI}$ for postnatal SGN function}

Despite the morphological data, deletion of Pou $4 f 1$ using the ectopic Atoh $1^{\text {cre }}$ mouse did not have 
any effect on number of SGNs, overall SG development or synaptic distribution. This result is not particularly surprising in light of a recent paper exploring the role of Pou4fl in the retina (Huang et al., 2014) in which a tamoxifen inducible Cre was used to knockout Pou4f1 after specification of retinal ganglion cells. The results from that study reported no change in ganglion cell number or morphology even six months after tamoxifen application. Furthermore, the conditional knockout mice still showed expression of Vglut2, which shows correlated expression with Pou4f1 in both retina and cochlea (Sherrill and Kelley, unpublished) suggesting that Pou4f1 may not be the sole or primary driver of cell identity in either retinal or spiral ganglion neurons.

Finally, while many studies have shown that synaptic location strongly correlates with spontaneous rate, as yet there is no data proving how the two features are linked mechanistically. Given the strong correlation between Pou4f1 expression and synaptic location, a next logical step would be to characterize possible changes in the physiological responses of Pou4f1-deleted SGNs

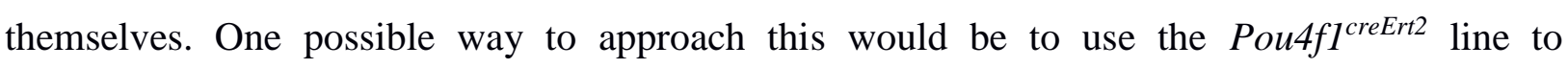
simultaneously delete Pouffl and fluorescently-label the same neurons. However, in contrast with the results reported in Petitpre et al. (2018), we observed limited overlap between SGNs that were labeled in response to sparse induction of Pou4f1 $1^{\text {creErt2 }}$ and immunolocalization of Pou4f1. The differing results could be a function of the differences in timing of induction. For our experiments induction occurred during the first post-natal week. In contrast, Petitpre et al. (2018) induced at approximately P25. Further, it has not been determined whether the Pou4f1 ${ }^{\text {creErt2 }}$ line faithfully recapitulates Pou4f1 expression in other regions of the CNS. The original transgenic construct used only the upstream $11 \mathrm{~kb}$ region of Pou4f1 which leaves open the possibility for non-specific activation as a result of the absence of more distant regulatory elements.

In conclusion, following an initial period of broad expression in all developing SGNs, Pou4f1 is down-regulated in approximately $70 \%$ of SGNs during the first post-natal week. The $30 \%$ of Type I SGNs that maintain expression of Pou4f1 into adulthood predominantly form synapses on the modiolar sides of IHCs. This result suggests that Pou4f1+ SGNs probably represent a sub-group of low-spontaneous rate, high threshold fibers. Deletion of Pou4f1 in SGNs leads to changes in the presynaptic AZs in IHCs, in particular causing a hyperpolarized activation range for their $\mathrm{Ca}^{2+}$ channels by increasing their voltage sensitivity. While these results demonstrate a role for post-synaptic SGNs in the function of presynaptic sites, more work remains to be done to elucidate the genetic factors that influence specification of physiologically distinct SGNs. 


\section{Materials and Methods}

\section{Animals}

All mice were maintained according to guidelines established by the joint NINDS/NIDCD IACUC at NIH. Most mice were maintained as mixed strain of predominately CD1 and C57-B6 background Both the Pou $4 f 1^{\text {flox }}$ and Pou $4 \mathrm{fl}^{-+-}$mice were obtained from Tudor Badea, NEI (Badea et al 2009, Xiang et al 1996). Ngn1 ${ }^{\text {creErt2 }}$ were obtained from Lisa Goodrich, Harvard Medical School (Koundakjian et al., 2007). Atohl $1^{\text {cre }}, R 26 R^{\text {tdTomato }}$ lines were obtained from Jackson Laboratories. Plugs were assumed to occur at midnight, and so mid-day on the day that a plug was observed was considered to be E0.5. Embryos were collected in PBS from euthanized pregnant dams. Post-natal pups were euthanized with $\mathrm{CO}_{2}$ followed by decapitation. Cochleae were collected and fixed in $4 \%$ paraformaldehyde in PBS for 1 hour at room temperature. Tissue requiring a short fixation were exposed to 4\% PFA in PBS for 20 minutes. P14 cochlea were decalcified in 10\% EDTA ( $\mathrm{pH} 7.4$ ) in PBS for 48 hours. For cryo-sectioning, cochleae were cryoprotected through a gradient of sucrose $(5 \%, 15 \%, 20 \%, 25 \%, 30 \%)$, and embedded and frozen in OCT embedding medium (Fisher). Sections $10 \square \mathrm{m}-14 \square \mathrm{m}$ thick were collected onto slides and allowed to dry at room temperature overnight before being frozen at $-80 \square \mathrm{C}$.

\section{Tamoxifen regime}

Pregnant dams were given a combined single dose of $0.2-0.5 \mathrm{~g} / 40 \mathrm{~g}$ body weight tamoxifen (T5648; Sigma-Aldrich) and 2g progesterone diluted in sunflower and flax oil by oral gavage on one day between E9.5-E12.5.

\section{Immunolabeling of fixed tissue}

Both whole cochlea and cryo-sectioned tissue were permeabilized and blocked in $0.05 \%$ Triton X-100 in PBS with 10\% normal serum (goat or horse, depending on the species the secondary was raised in) for 1 hour at room temperature. If the primary antibody was raised in mouse, the blocking solution would also include $0.5 \%$ Unconjugated AffiniPure FAB fragment goat Antimouse IgG $(\mathrm{H}+\mathrm{L})$ (Jackson ImmunoResearch Labs). Cochleae were washed 3 times for 5 minutes with PBS. Tissue was then incubated with the appropriate concentration of primary antibody in $0.05 \%$ Triton X-100 in PBS overnight at $4 \square$ C. Tissue was then washed 3 times for 10 minutes to 
ensure primary antibody was thoroughly removed. Species-appropriate Alexa-conjugated secondary antibodies (1:1000) were then incubated with the tissue for 1 hour at room temperature. When appropriate, DAPI (300 $\mathrm{nM})$ was applied to whole cochleae for 5 minutes at room temperature. Tissue was washed 3 times for 10 minutes each. Whole cochlear tissue was divided into basal and apical pieces and then mounted in Fluoromount-G (SouthernBiotech). Sectioned tissue was mounted in DAPI Fluoromount-G or Fluoromount-G (SouthernBiotech). For sectioned tissue stained using the anti-brn3a (Pou4f1) antibody, antigen retrieval was performed prior to immunolabeling. Briefly, slides were placed on a platform above the boiling water in a commercially purchased rice cooker and covered with sodium citrate buffer (10mM sodium citrate, $0.05 \%$ tween, $\mathrm{pH}$ 6.0). Slides were steamed in the sodium citrate buffer for 10 minutes and then allowed to cool for 10 minutes. Slides were then washed in PBS 3 times and immunolabeling was performed as above. The following primary antibodies were used: Anti-Pou4f1 (Millipore; MAB1585), Anti-Myosin7a (Proteus Biosciences; 25-6790), Anti-CtBP2 (Santa Cruz Biotechnology; SC5967), anti-Beta-Tubulin 3 (Tuj1; Sigma-Aldrich; T2200), AntiNeurofilament Heavy (Aves Labs; NFH), Anti-tdTomato (Kerafast; 16D7).

\section{Patch-clamp and live-confocal $\mathrm{Ca}^{2+}$-imaging}

The apical 2/3 turns of organs of Corti from P21-28 old mice were freshly dissected in HEPES Hank's solution containing (in mM): $5.36 \mathrm{KCl}, 141.7 \mathrm{NaCl}, 10 \mathrm{HEPES}, 0.5 \mathrm{MgSO}_{4} 7 \mathrm{H}_{2} \mathrm{O}, 1$ $\mathrm{MgCl}_{2}-6 \mathrm{H}_{2} \mathrm{O}, 1 \mathrm{mg} / \mathrm{ml}$ D-glucose, and $0.5 \mathrm{mg} / \mathrm{ml}$ L-glutamine (pH 7.2, 300 mOsm). The native morphologies and positions of the IHCs within the organ of Corti were maintained as much as possible, by exposing the modiolar face of the IHC and carefully removing the surrounding cells with a suction pipette. All experiments were conducted at room temperature $\left(20-25^{\circ} \mathrm{C}\right)$. The patch pipette solution contained (in mM): $111 \mathrm{Cs}$-glutamate, $1 \mathrm{MgCl}_{2}, 1 \mathrm{CaCl}_{2}, 10$ EGTA, 13 TEA-Cl, 20 HEPES, 4 Mg-ATP, 0.3 Na-GTP and 1 L-Glutathione (pH 7.3, 290 mOsm). To visualize the $\mathrm{Ca}^{2+}$-hotspots and the ribbons, the $\mathrm{Ca}^{2+}$-indicator Fluo-4FF penta-K ${ }^{+}$salt $(0.8 \mathrm{mM}$, Life Techologies, Germany) and the TAMRA-conjugated CtBP2/RIBEYE-binding dimer peptide (10 $\mu \mathrm{M}$, Biosynthan, Germany) were added to the intracellular solution just before experiment. The extracellular solution contained the following (in $\mathrm{mM}$ ): $2.8 \mathrm{KCl}, 102 \mathrm{NaCl}, 10 \mathrm{HEPES}, 1 \mathrm{CsCl}_{2}$, $1 \mathrm{MgCl}_{2}, 5 \mathrm{CaCl}_{2}, 35 \mathrm{TEA}-\mathrm{Cl}$, and $2 \mathrm{mg} / \mathrm{ml}$ D-Glucose (pH 7.2, 300 mOsm). An EPC-10 amplifier controlled by Patchmaster (HEKA Elektronik, Germany) was used for the acquisition. 
IHCs were held at $-87 \mathrm{mV}$. All voltages were corrected offline for liquid junction potential (-17 $\mathrm{mV}$ ) and voltage-drops across the series resistance $\left(\mathrm{R}_{\mathrm{s}}\right)$. Recordings were discarded when the leak current exceeded $-50 \mathrm{pA}, \mathrm{R}_{\mathrm{s}}$ exceeded $15 \mathrm{M} \Omega$ within 4 min after break-in, or $\mathrm{Ca}^{2+}$-current rundown exceeded $25 \%$. $\mathrm{Ca}^{2+}$-imaging was performed with a spinning disk confocal scanner (CSU22, Yokogawa, Japan) mounted on an upright microscope (Axio Examiner, Zeiss, Germany) with 63x, 1.0 NA objective (W Plan-Apochromat, Zeiss). Images were acquired by a scientific CMOS camera (Neo, Andor, Ireland). $\mathrm{Ca}^{2+}$-indicator F4FF and TAMRA-conjugated peptide were excited by diode-pumped solid-state lasers with $491 \mathrm{~nm}$ and $561 \mathrm{~nm}$ wavelength, respectively (Cobolt AB, Sweden). The spinning disk was set to $2000 \mathrm{rpm}$ to synchronize with the $100 \mathrm{~Hz}$ acquisition rate of the camera.

Using a piezo positioner for the objective (Piezosysteme Jena, Germany), a scan of the entire cell was performed 4 min after breaking into the cell, taking sections each $0.5 \mu \mathrm{m}$ at an exposure time of $0.5 \mathrm{~s}$ in the red (TAMRA-peptide) channel from the bottom to the top of the cell. In order to study the voltage-dependence of $\mathrm{Ca}^{2+}$-indicator fluorescence increments at the synapses, the confocal scans were acquired every $0.5 \mu \mathrm{m}$ from the bottom-most to the top-most ribbon. $\mathrm{Ca}^{2+}$-currents were evoked by applying a voltage ramp stimulus from -87 to $+63 \mathrm{mV}$ during $150 \mathrm{~ms}(1 \mathrm{mV} / \mathrm{ms})$ in each focal plane. Simultaneously, fluorescence measurements were made in the green channel (Fluo-4FF) with a frame rate of $100 \mathrm{~Hz}$. In order to overcome the limitations of the frame rate and increase the voltage resolution of the fluorescent signal acquired, the voltage ramp protocol was applied twice, once shifted by $5 \mathrm{~ms}$ such that for any given frame during the second ramp the voltage was shifted by $5 \mathrm{mV}$ compared to the first stimulus. Alternating planes were acquired to avoid photobleaching encountered with the consecutive plane acquisitions.

\section{Data analysis}

\section{Live-imaging and IHC-patch-clamp}

The data were analyzed using custom programs in Igor Pro 6.3 (Wavemetrics, Portland, OR, USA). For analysis of IV-curves, the evoked $\mathrm{Ca}^{2+}$-currents were averaged from 5 to $10 \mathrm{~ms}$ after the start of the depolarization. $\Delta \mathrm{F}$ images were created by subtracting the fluorescence intensities inside the cell at resting state $\left(\mathrm{F}_{0}\right.$, average of 10 frames $)$ from the ones at the depolarized state (average of 6 frames during voltage ramp protocol). $\Delta \mathrm{F}$ for each hotspot was calculated as the average of a 9 pixel square placed in the region exhibiting the greatest intensity increase within 
the fluorescent hotspot. The fluorescence-voltage relationship (FV) was sorted by the corresponding voltage of each fluorescence intensity value. Maximal $\Delta \mathrm{F}\left(\Delta \mathrm{F}_{\max }\right)$ was the average of $5 \Delta \mathrm{F}$ values obtained during the voltage ramp protocol (at the peak of $\mathrm{Ca}^{2+}$ influx). Only fluorescent increments presenting a $\Delta \mathrm{F}_{\max }$ greater than the average of the fluorescence intensity plus 2 standard deviations at rest were defined as synaptic $\mathrm{Ca}^{2+}$ signals and considered for further analysis. Due to their noisiness, the raw FV traces were refined by being fitted to the following function in order to analyze their voltage dependence:

$$
F(V)=F_{0}+\frac{f_{v} \cdot\left(V_{r}-V\right)}{1+e^{\frac{\left(V_{h}-V\right)}{k}}}
$$

where $V$ stands for the voltage command. The fitting parameters were determined by Igor Pro automatically, and their initial guess resulted from the calculations of $\mathrm{F}_{0}$ the signal at rest, $V_{h}$ for the voltage value of half-maximal activation and $k$ for the voltage sensitivity obtained from a sigmoid fitting, and the slope factor $f_{v}$ obtained by the linear fitting of the FV-trace in the range of 3 to $23 \mathrm{mV}$, where the decrease of fluorescence at positive voltages results from the declining driving force despite full activation of the $\mathrm{Ca}^{2+}$ channels. The resulting fitting trace was forced to reach the reversal potential $V_{r}$, optimally calculated from the $\mathrm{Ca}^{2+}$-currents. The FV fitting trace was then divided by the $f_{v}$. line extended to all the corresponding voltages, to estimate the assumed fluorescence intensity of every voltage in the full activation condition, giving the fractional activation curves. The fractional activation curves were then fitted by the Boltzmann function to obtain the voltage for half activation $\left(\mathrm{V}_{\mathrm{h}}\right)$ and slope-factor $(\mathrm{k})$. Synaptic ribbon fluorescence (visualized with the TAMRA-conjugated CtBP2/RIBEYE-binding dimer peptide) was estimated by measuring the ratio of the strongest fluorescence pixel intensity to that of the pixel nearby (89 pixels away inside the cell) $\left(\mathrm{F}_{\text {ribbon }} / \mathrm{F}_{\text {nearby }}\right)$. In order to combine live-imaging data from multiple cells, we reconstructed the morphology of individual IHCs and the positions of their synapses based on the fluorescence of the TAMRA-conjugated RIBEYE-binding peptide and then transformed the Cartesian coordinates into cell-aligned cylindrical coordinates (for more details see Ohn et al., 2016). In brief, for each cell we identified the plane of symmetry orthogonal to the tonotopic axis. Then we sectioned the IHC orthogonally to a straight line fitting the pillar edge of the cell in the plane of symmetry. We calculated the center of mass for each section and connected those of the bottom-most and of the largest section to define the central axis for our cylindrical coordinate system. We projected the AZ coordinates of multiple cells along their central axis for the polar charts, with the 4 sides annotated as modiolar or pillar (facing toward or away from the 
ganglion), and apical or basal (toward the cochlear tonotopic apex or base).

\section{$\underline{\text { Immunostaining }}$}

To analyze the intensity and position of ribbons in confocal images of fixed whole-mount organs of Corti, a customized algorithm was developed as a program in MATLAB software and used as a plug-in in Imaris software. The locations of the ribbons were defined as the centers of mass of CtBP2-immunofluorescent spots by fitting Gaussian functions in three dimensions. The spots were selected by a subjective criterion of the quality of the fitting. Immunofluorescence intensities were measured as the sum of the voxel values within a defined region (3 voxels in $\mathrm{X}$, $\mathrm{Y}$, and 2 in $\mathrm{Z}$ ) with the center of mass of the spot as origin. The cytosolic staining allowed us to assign each ribbon to an IHC. After marking the center of each IHC nucleus, a vector passing by this point and defining the central axis of the cylindrical model of the cell was placed by being adjusted to the relative orientation of each IHC cell in the XY and YZ axis. The Cartesian coordinates of the ribbons were transformed to cell-centric cylindrical coordinates in order to adjust differences in cellular orientation relative to the $\mathrm{XYZ}$ axes of the microscope. Intensities of CtBP2 puncta as a function of their positions could be analyzed for multiple cells by overlaying their central axes with alignment to the center of each nucleus (for more details see Ohn et al., 2016).

\section{Statistical analysis}

The data were analyzed using Matlab (Mathworks), Excel, Igor Pro 6 (Wavemetrics). Averages were expressed as mean \pm standard error of the mean (S.E.M.). For every dataset the standard deviation (S.D.), number of replicates $(n)$ and animals $(\mathrm{N})$ were indicated. In order to compare two samples, data sets were tested for normal distribution (Jarque-Bera test) and equality of variances (F-test) followed by two-tailed unpaired Student's t-test, or, when data were not normally distributed and/or variance was unequal between samples, the unpaired two-tailed Mann-Whitney-Wilcoxon test was used. The non-significant difference between samples is reported as n.s., significant differences are reported as $* p<0.05, * * p<0.01$, *** $p<0.001$, **** $p<0.0001$. 


\section{References}

Badea TC, Cahill H, Ecker J, Hattar S, Nathans J (2009a) Distinct roles of transcription factors brn $3 \mathrm{a}$ and brn $3 \mathrm{~b}$ in controlling the development, morphology, and function of retinal ganglion cells. Neuron 61:852-864.

Badea TC, Hua ZL, Smallwood PM, Williams J, Rotolo T, Ye X, Nathans J (2009b) New mouse lines for the analysis of neuronal morphology using CreER(T)/loxP-directed sparse labeling. PLoS One 4:e7859.

Blanchard JW, Eade KT, Szucs A, Lo Sardo V, Tsunemoto RK, Williams D, Sanna PP, Baldwin KK (2015) Selective conversion of fibroblasts into peripheral sensory neurons. Nat Neurosci $18: 25-35$.

Coate TM, Spita NA, Zhang KD, Isgrig KT, Kelley MW (2015) Neuropilin-2/Semaphorin-3Fmediated repulsion promotes inner hair cell innervation by spiral ganglion neurons. Elife 4.

Deng M, Yang H, Xie X, Liang G, Gan L (2014) Comparative expression analysis of POU4F1, POU4F2 and ISL1 in developing mouse cochleovestibular ganglion neurons. Gene Expr Patterns 15:31-37.

Frank T, Khimich D, Neef A, Moser T (2009) Mechanisms contributing to synaptic Ca2+ signals and their heterogeneity in hair cells. Proc Natl Acad Sci U S A 106:4483-4488.

Hua ZL, Emiliani FE, Nathans J (2015) Rac1 plays an essential role in axon growth and guidance and in neuronal survival in the central and peripheral nervous systems. Neural Dev 10:21.

Huang EJ, Liu W, Fritzsch B, Bianchi LM, Reichardt LF, Xiang M (2001) Brn3a is a transcriptional regulator of soma size, target field innervation and axon pathfinding of inner ear sensory neurons. Development 128:2421-2432.

Huang L, Hu F, Xie X, Harder J, Fernandes K, Zeng XY, Libby R, Gan L (2014) Pou4f1 and pou $4 \mathrm{f} 2$ are dispensable for the long-term survival of adult retinal ganglion cells in mice. PLoS One 9:e94173.

Jean P, Lopez de la Morena D, Michanski S, Jaime Tobon LM, Chakrabarti R, Picher MM, Neef J, Jung S, Gultas M, Maxeiner S, Neef A, Wichmann C, Strenzke N, Grabner C, Moser T (2018) The synaptic ribbon is critical for sound encoding at high rates and with temporal precision. Elife 7 .

Jehn B, Chicaiza G, Martin F, Jaggi R (1994) Isolation of three novel POU-domain containing cDNA clones from lactating mouse mammary gland. Biochemical and biophysical research communications 200:156-162.

Koundakjian EJ, Appler JL, Goodrich LV (2007) Auditory neurons make stereotyped wiring decisions before maturation of their targets. J Neurosci 27:14078-14088.

Liberman LD, Liberman MC (2016) Postnatal maturation of auditory-nerve heterogeneity, as seen in spatial gradients of synapse morphology in the inner hair cell area. Hear Res 339:12-22. 
Liberman LD, Wang H, Liberman MC (2011) Opposing gradients of ribbon size and AMPA receptor expression underlie sensitivity differences among cochlear-nerve/hair-cell synapses. J Neurosci 31:801-808.

Liberman MC (1978) Auditory-nerve response from cats raised in a low-noise chamber. J Acoust Soc Am 63:442-455.

Lumpkin EA, Collisson T, Parab P, Omer-Abdalla A, Haeberle H, Chen P, Doetzlhofer A, White P, Groves A, Segil N, Johnson JE (2003) Math1-driven GFP expression in the developing nervous system of transgenic mice. Gene Expr Patterns 3:389-395.

Malik V, Zimmer D, Jauch R (2018) Diversity among POU transcription factors in chromatin recognition and cell fate reprogramming. Cell Mol Life Sci 75:1587-1612.

Matei V, Pauley S, Kaing S, Rowitch D, Beisel KW, Morris K, Feng F, Jones K, Lee J, Fritzsch B (2005) Smaller inner ear sensory epithelia in Neurog 1 null mice are related to earlier hair cell cycle exit. Dev Dyn 234:633-650.

Merchan-Perez A, Liberman MC (1996) Ultrastructural differences among afferent synapses on cochlear hair cells: correlations with spontaneous discharge rate. J Comp Neurol 371:208-221.

Meyer AC, Frank T, Khimich D, Hoch G, Riedel D, Chapochnikov NM, Yarin YM, Harke B, Hell SW, Egner A, Moser T (2009) Tuning of synapse number, structure and function in the cochlea. Nat Neurosci 12:444-453.

O'Donovan KJ, Ma K, Guo H, Wang C, Sun F, Han SB, Kim H, Wong JK, Charron J, Zou H, Son YJ, He Z, Zhong J (2014) B-RAF kinase drives developmental axon growth and promotes axon regeneration in the injured mature CNS. J Exp Med 211:801-814.

Ohlemiller KK, Echteler SM, Siegel JH (1991) Factors that influence rate-versus-intensity relations in single cochlear nerve fibers of the gerbil. J Acoust Soc Am 90:274-287.

Ohn TL, Rutherford MA, Jing Z, Jung S, Duque-Afonso CJ, Hoch G, Picher MM, Scharinger A, Strenzke N, Moser T (2016) Hair cells use active zones with different voltage dependence of Ca2+ influx to decompose sounds into complementary neural codes. Proc Natl Acad Sci U S A 113:E4716-4725.

Pangrsic T, Singer JH, Koschak A (2018) Voltage-Gated Calcium Channels: Key Players in Sensory Coding in the Retina and the Inner Ear. Physiol Rev 98:2063-2096.

Petitpre C, Wu H, Sharma A, Tokarska A, Fontanet P, Wang Y, Helmbacher F, Yackle K, Silberberg G, Hadjab S, Lallemend F (2018) Neuronal heterogeneity and stereotyped connectivity in the auditory afferent system. Nature communications 9:3691.

Sajgo S, Ghinia MG, Brooks M, Kretschmer F, Chuang K, Hiriyanna S, Wu Z, Popescu O, Badea TC (2017) Molecular codes for cell type specification in Brn3 retinal ganglion cells. Proc Natl Acad Sci U S A 114:E3974-E3983. 
Shrestha BR, Chia C, Wu L, Kujawa SG, Liberman MC, Goodrich LV (2018) Sensory Neuron Diversity in the Inner Ear Is Shaped by Activity. Cell 174:1229-1246 e1217.

Sun S, Babola T, Pregernig G, So KS, Nguyen M, Su SM, Palermo AT, Bergles DE, Burns JC, Muller U (2018) Hair Cell Mechanotransduction Regulates Spontaneous Activity and Spiral Ganglion Subtype Specification in the Auditory System. Cell 174:1247-1263 e1215.

Taberner AM, Liberman MC (2005) Response properties of single auditory nerve fibers in the mouse. J Neurophysiol 93:557-569.

Tantin D (2013) Oct transcription factors in development and stem cells: insights and mechanisms. Development 140:2857-2866.

Winter IM, Robertson D, Yates GK (1990) Diversity of characteristic frequency rate-intensity functions in guinea pig auditory nerve fibres. Hear Res 45:191-202.

Xiang M, Gan L, Zhou L, Klein WH, Nathans J (1996) Targeted deletion of the mouse POU domain gene Brn-3a causes selective loss of neurons in the brainstem and trigeminal ganglion, uncoordinated limb movement, and impaired suckling. Proc Natl Acad Sci U S A 93:1195011955.

Zenisek D, Horst NK, Merrifield C, Sterling P, Matthews G (2004) Visualizing synaptic ribbons in the living cell. J Neurosci 24:9752-9759. 
5 Chapter 3: Article in revision

\title{
"Intrinsic planar polarity mechanisms influence the
}

\section{position-dependent regulation of synapse properties in inner}

\section{hair cells"}

\author{
P. Jean, Ö. Demet Özcete, B. Tarchini and T. Moser
}

\section{Author contributions:}

T.M, B.T and P.J designed the study. P.J performed immunohistochemistry, confocal and STED immunofluorescence microscopy as well as patch-clamp recordings, $\mathrm{Ca}^{2+}$ imaging and analysis. B.T provided the mice, performed the immunostainings and confocal microscopy. Ö.D.Ö performed the patchclamp capacitance measurements and analysis. T.M supervised P.J. and Ö.Ö., T.M., P.J. and B.T. prepared the manuscript. 


\begin{abstract}
Encoding the wide range of audible sounds in the mammalian cochlea is collectively achieved by functionally diverse type I spiral ganglion neurons (SGNs) at each tonotopic position. The firing of each SGN is thought to be driven by an individual active zone (AZ) of a given inner hair cell (IHC). These AZs present distinct properties according to their position within the IHC, to some extent forming a gradient between the modiolar and the pillar IHC side. In this study, we investigated whether signaling involved in planar polarity at the apical surface can influence position-dependent AZs properties at the IHC base. Specifically, we tested the role of Gai proteins and their binding partner LGN/Gpsm2 implicated in cytoskeleton polarization and hair cell (HC) orientation along the epithelial plane. Using high and super-resolution immunofluorescence microscopy as well as patch-clamp combined with confocal calcium imaging we analyzed IHCs in which Gai signaling was blocked by Cre-induced expression of the pertussis toxin catalytic subunit (PTXa). PTXa-expressing IHCs exhibited larger Cav1.3 $\mathrm{Ca}^{2+}$-channel clusters and consequently greater $\mathrm{Ca}^{2+}$ influx at the whole-cell and single synapse levels, which also showed a hyperpolarized shift of activation. Moreover, PTXa expression collapsed the modiolar-pillar gradients of ribbon size and maximal synaptic $\mathrm{Ca}^{2+}$ influx. Finally, genetic deletion of Gai3 and LGN/Gpsm2 also disrupted the modiolar-pillar gradient of ribbon size. We propose a role for Gai proteins and LGN in regulating the position-dependent AZ properties in IHCs and suggest that this signaling pathway contributes to setting up the diverse firing properties of SGNs.
\end{abstract}

\title{
Significance statement
}

The wide dynamic range of sound encoding enables perception of sounds varying over six orders of magnitude, thought to be collectively achieved by distinct classes of type I spiral ganglion neurons (SGNs) contacting each inner hair cell (IHC). These synaptic contacts have been shown to vary according to the SGN firing properties along the modiolar-pillar axis. It has also been shown that the IHCs spatially segregate active zones (AZs) with different $\mathrm{Ca}^{2+}$ - influx properties, proposing an attractive mechanism responsible for the sound encoding heterogeneity among SGNs. Our study, combining confocal/STED immunofluorescence microscopy, patch-clamp and calcium imaging, proposes that Gai and LGN, involved in planar polarity mechanisms at the IHC apex, contribute to establish the spatial gradient of IHC AZ properties. 


\section{Introduction}

The sense of hearing relies on vivid, temporally precise and tireless encoding of sounds ranging in pressure over six orders of magnitude. Active amplification of cochlear vibrations for soft sounds and compression at strong ones enable the IHC receptor potential to represent the broad range of audible sound pressures (Russell and Sellick, 1983). Each IHC forms ribbon synapses with several type I SGNs that relay the auditory information to the brainstem. Each of the functionally diverse SGNs only encodes a fraction of the audible range of sound pressures. Collectively they cover the entire dynamic range: high spontaneous rate, low sound threshold SGNs (high SR) encode soft sounds and low spontaneous rate, high threshold SGNs (low SR) loud sounds (Liberman, 1978; Ohlemiller et al., 1991; Winter et al., 1990). Since such functionally diverse SGNs can exhibit comparable tuning frequency, they are thought to receive input from neighboring or even the same IHC at a given tonotopic position (Liberman, 1982, 1978; Merchan-Perez and Liberman, 1996). Based on backtracing experiments it was proposed that low SR SGNs preferentially contact the modiolar side of the IHCs, facing the spiral ganglion, while high SR SGNs are more likely to target the pillar side of the IHCs, facing the outer hair cells (OHCs) (Merchan-Perez and Liberman, 1996).

Several mechanisms have been proposed to explain the different SGN firing patterns. Postsynaptic morphology shows different fiber caliber and mitochondrial content (Merchan-Perez and Liberman, 1996) as well as different amounts of AMPA receptors (Liberman et al., 2011; Yin et al., 2014; Zhang et al., 2018). The lateral olivocochlear system could provide differential efferent modulation (Ruel et al., 2001; Yin et al., 2014). Finally, presynaptic mechanisms could establish the different firing properties of SGNs (Frank et al., 2009; Kantardzhieva et al., 2013; Merchan-Perez and Liberman, 1996; Meyer et al., 2009; Ohn et al., 2016), where larger ribbons associated with more synaptic vesicles and $\mathrm{Ca}^{2+}$ channels are found at modiolar AZs likely facing low SR SGNs (Frank et al., 2009; Kantardzhieva et al., 2013; Merchan-Perez and Liberman, 1996; Meyer et al., 2009; Ohn et al., 2016). How larger AZs, likely having greater maximal synaptic strength could drive low SR SGNs remained a mystery until it was discovered that their $\mathrm{Ca}^{2+}$ influx seems to operate at more depolarized potentials than that of AZs of the pillar side (Ohn et $a l ., 2016)$. This could readily explain the higher spontaneous rate and lower sound threshold of the SGNs contacting the pillar AZs, where substantial release is expected at the resting potential of IHCs and small receptor potentials might suffice to increase the release rate (Ohn et al., 2016). However, how the IHC manages to diversify AZs to decompose the full auditory information 
contained in the receptor potential into different neural channels remains largely unclear. Nonmutually exclusive candidate mechanisms for the observed spatial gradients of AZ properties include: i) a cell autonomous signaling mechanism in IHCs, or an instructive influence by ii) SGNs or iii) efferent innervations.

Here, we explored the exciting possibility that planar polarity mechanisms responsible for establishing the proper architecture of the hair bundles and their uniform orientation across HCs (Ezan et al., 2013; Goodrich and Strutt, 2011; Lu and Sipe, 2016) also instruct the observed basolateral gradients of IHC synapse properties. During development, the apical HC compartment located between the tallest stereocilia and the abneural junctions of the cell (the "bare zone") lacks protrusions and hosts the polarized localization of mInsc, LGN/Gpsm2 and Gai (Tarchini et al., 2013). This protein complex was first shown to control mitotic spindle orientation and thereby asymmetric cell division, a broadly conserved function including in mammals (Bergstralh et al., 2017). Gai-LGN exclude aPKC and the resulting compartmentalization of the HC apex influences the position of the kinocilium and the stereocilia, shaping cytoskeleton intrinsic asymmetry and possibly aligning it with tissue-wide polarity cues during early development (Ezan et al., 2013; Tarchini et al., 2013). Moreover, LGN and Gai are also enriched at the tips of the tallest stereocilia (Mauriac et al., 2017; Tarchini et al., 2016), making the mInsc/LGN/Gai complex a promising candidate for conveying information across compartments (Tarchini et al., 2016). Pertussis toxin (PTX) is well-known to ADP-ribosylate and block Gai signaling, and interestingly, PTX application led to planar cell polarity (PCP) defects and a deficit of kinocilium migration in HC in cochlear cultures (Ezan et al., 2013). Moreover, transgenic or Cre-induced expression of PTX catalytic subunit (PTXa) in HC in vivo induced PCP defects, stereocilia stunting and severe hair bundle disorganization associated to profound deafness, similar to the loss of LGN (Tarchini et al., 2013, 2016). Milder hair bundle defects and hearing loss were also reported in mouse mutants lacking Gai3 (Mauriac et al., 2017), and these phenotypes were accentuated in conditional double Gai2; Gai3 mutants (Beer-Hammer et al., 2018).

Here we combined morphological and physiological analysis of mouse IHCs expressing PTXa (Tarchini et al., 2016) and found that the disruption of Gai signaling affects the spatial heterogeneity and properties of the AZs in IHCs. Confocal and STED microscopy showed that the mutant synapses were unchanged in number, but were reorganized with larger and more complex $\mathrm{Ca}^{2+}$ channel clusters and a loss of the modiolar-pillar gradient of ribbon size. Performing patch-clamp and live confocal $\mathrm{Ca}^{2+}$ imaging, we showed that the mutant IHCs exhibited an 
increased amplitude and hyperpolarized activation of $\mathrm{Ca}^{2+}$ influx at the whole-cell and single synapse levels. Moreover, the modiolar-pillar gradient for synaptic $\mathrm{Ca}^{2+}$ influx strength observed in control condition was collapsed in PTXa-expressing IHCs. The gradient of ribbon size was also disrupted in Gai3 and LGN KOs, suggesting that HC-intrinsic mechanisms influencing planar polarity at the apical membrane might also influence the asymmetric modiolar-pillar distribution of ribbon synapse properties.

\section{Results}

\section{The modiolar/pillar gradient of ribbon size is dependent on Gai signaling}

Organs of Corti from 3-week-old control (Atoh1-Cre- or PTXa-negative, referred to as PTXa controls) and Atoh1-Cre; PTXa ${ }^{\mathrm{fl} / \mathrm{fl}}$ mice (referred to as PTXa mutants) were processed in parallel for immunohistochemistry. As previously reported (Tarchini et al., 2016), PTXa-IHCs presented disorganized and shortened hair bundles. Moreover, they occasionally showed a misplaced basal body indicated by the position of the fonticulus/cytoplasmic channel in phalloidin stainings of Factin, demonstrating that planar orientation defects most severe in OHCs can also affect IHCs. In control IHCs, the fonticuli were invariably located towards the pillar side of the cell, indicating a correct IHC orientation. Interestingly, most misoriented IHCs showed a diametrically opposite position of the fonticulus towards the modiolus ( $180^{\circ}$ inversion, Figure $\left.1 \mathrm{~A}\right)$.

Combining F-actin labeling to record IHC orientation with immunostaining against Otoferlin or Vglut3 to outline the IHC shape and CtBP2/RIBEYE to estimate the localization and size of the ribbons, we investigated whether this inversion of orientation might influence the modiolar-pillar gradient of ribbon size previously reported (Liberman and Liberman, 2016; Ohn et al., 2016) in IHCs. For analysis, we reconstructed the IHCs in cylindrical coordinates to overlay multiple cells and study the position-dependence of ribbon size (see Methods). As expected, in IHCs of PTXa control littermates, the modiolar-pillar gradient of RIBEYE-immunofluorescence intensity (proxy of "ribbon size" (Wong et al., 2014), normalized to the mean modiolar RIBEYE immunofluorescence intensity) was observed (modiolar: $1.00 \pm 0.03$ (S.D. $=0.40$ ), $n$ (number of replicates $)=211$ AZs vs. pillar: $0.82 \pm 0.03($ S.D. $=0.34), n=148$ AZs in 22 IHCs, $\mathrm{N}$ (number of animals) $=5, p<0.00001$, Mann-Whitney-Wilcoxon test) (Figure $1 \mathrm{~B}$ and C, in black). Initially, we considered using PTXa-expressing, non-inverted IHCs as internal controls. Interestingly however, we observed a complete collapse of the ribbon size gradient upon PTXa expression 
regardless of IHC orientation (PTXa mutant non-inverted, in blue: modiolar, $1.00 \pm 0.03$ (S.D. = 0.35), $n=190$ AZs vs. pillar, $0.99 \pm 0.03$ (S.D.=0.35), $n=171$ AZs in 22 IHCs, $\mathrm{N}=12, p=0.88$; PTXa mutant $180^{\circ}$ inverted, in red: modiolar, $1.00 \pm 0.02$ (S.D.=0.31), $n=192$ AZs vs. pillar, $0.95 \pm 0.02(\mathrm{~S} . \mathrm{D} .=0.31) n=174 \mathrm{AZs}$ in $22 \mathrm{IHCs}, \mathrm{N}=12, p=0.75$ Mann-Whitney-Wilcoxon test for both conditions) (Figure 1B and C).

To enhance the specificity of our manipulation, we turned to mutants lacking Gai3. While heterozygote littermate controls presented the expected modiolar-pillar gradient of RIBEYEimmunofluorescence intensity $\left(\mathrm{G \alpha i}^{\mathrm{KO} /+}\right.$ :modiolar: $1.00 \pm 0.02$ (S.D. $\left.=0.33\right), n=225 \mathrm{AZs}$ vs. pillar: $0.76 \pm 0.02$ (S.D. $=0.27) n=143 \mathrm{AZs}$ for 20 IHCs, $\mathrm{N}=5, p<0.00001$, Mann-WhitneyWilcoxon test), homozygote mutants lost this gradient $\left(\right.$ Gai $3^{K O / K O}$ : modiolar: $1.00 \pm 0.02$ (S.D. = 0.36), $n=218$ AZs vs. pillar: $1.04 \pm 0.03$ (S.D. =0.38) $n=145$ AZs for $20 \mathrm{IHCs}, \mathrm{N}=5, p=0.24$, Mann-Whitney-Wilcoxon test) (Figure 2A). Interestingly, inactivating LGN/Gpsm2, a direct binding partner of Gai3, also collapsed the gradient of ribbon size ( $L G N^{K O / K O}$ : modiolar: $1.00 \pm$ 0.02 (S.D. $=0.35$ ), $n=231$ AZs vs. pillar: $0.96 \pm 0.03$ (S.D. =0.36) $n=184 \mathrm{AZs}, p=0.11$ vs. $L G N^{K O /+}$ : modiolar: $1.00 \pm 0.02$ (S.D. $\left.=0.36\right), n=298$ AZs vs. pillar: $0.75 \pm 0.02$ (S.D. = 0.27), $n=128$ AZs, $p<0.00001 ; 24$ IHCs, $\mathrm{N}=4$, Mann-Whitney-Wilcoxon test for both conditions) (Figure 2B). Together, these results suggest that the Gai-LGN complex previously shown to affect cell polarity during apical $\mathrm{HC}$ morphogenesis is also critical to establish the modiolar-pillar gradient of ribbon synapse size in IHCs.

Down-regulating Gai activity in HCs with PTXa, inactivating both Gai2+Gai3 or inactivating LGN resulted in profound deafness in young adults already (Beer-Hammer et al., 2018; Tarchini et al., 2016). To explore the possibility that the disruption of the modiolar-pillar gradient of ribbon size is consecutive to the loss of sound-evoked synaptic activity, we turned to Myo1 $5^{\text {sh } 2}$ mice. Myo $15^{\text {sh } 2}$ HCs show normal planar architecture and orientation, but like PTXa and LGN mutants exhibit disorganized and shortened hair bundles associated with complete deafness (Probst et al., 1998). Myo15 $5^{\text {sh/sh2 }}$ IHCs retained a significant modiolar-pillar gradient of ribbon size $\left(\right.$ Myo15 ${ }^{\text {sh/sh2}}$ : Modiolar: $1.00 \pm 0.05$ (S.D. $\left.=0.65\right), n=173$ AZs vs. pillar: $0.66 \pm 0.04$ (S.D.= 0.62), $n=133$ AZs for $21 \mathrm{IHCs}, \mathrm{N}=3 ;$ Myo1 $^{\text {sh2/ }}{ }^{+}$controls: Modiolar: $1.00 \pm 0.03$ (S.D. = 0.37), $n=203$ AZs vs. pillar:0.73 \pm 0.02 (S.D.=0.23), $n=193$ AZs for $21 \mathrm{IHCs}, \mathrm{N}=3, p<0.00001$ and Mann-Whitney-Wilcoxon test for both conditions) (Figure supplement 1). This indicates that altered hair bundle function resulting from stunted stereocilia is not the primary cause for the lost ribbon size gradient observed in IHCs with defective Gai or LGN function. 


\section{Disrupting Gai signaling reorganizes and potentiates $\mathrm{Ca}^{2+}$-influx at IHC AZs}

Next, we performed a detailed immunofluorescence analysis of synapse morphology in PTXaexpressing IHCs. We stained against CtBP2/RIBEYE and found no significant differences between PTXa control and mutant conditions for the average number of ribbons per IHC (PTXa mutant: $12.41 \pm 0.43$ (S.D. $=2.12)$ vs. PTXa control: $12.54 \pm 0.29 .($ S.D. $=1.44) ; n=24$ IHCs, $\mathrm{N}$ $=6, p=0.81$, t-test) or their mean RIBEYE immunofluorescence intensity (PTXa mutant: $6.51 \pm$ 0.13 a.u. (S.D. $=2.49$ a.u.) $n=360 \mathrm{AZs,} \mathrm{N}=9$ vs. PTXa control: $6.62 \pm 0.13$ a.u. (S.D. $=2.26$ a.u.); $n=327 \mathrm{AZs} ; \mathrm{N}=9, p=0.28$, Mann-Whitney-Wilcoxon) (Figure supplement 2A, B and C). Next, we labeled Cav1.3 $\mathrm{Ca}^{2+}$ channels, contributing more than $90 \%$ of the voltage-gated $\mathrm{Ca}^{2+}$ influx in IHCs (Brandt et al., 2003; Dou et al., 2004; Platzer et al., 2000). Using confocal microscopy, presynaptic $\mathrm{Ca}^{2+}$-channel clusters were identified as spots of $\mathrm{Ca}_{\mathrm{v}} 1.3$ immunofluorescence juxtaposed to the postsynaptic density (PSD) detected as PSD-95 immunofluorescent spots. Cav1.3-channels remained clustered at mutant AZs and were further quantified by fitting Cav1.3 immunofluorescent spots with a 2D-Gaussian function. The amplitudes of the fits in PTXa-expressing IHCs were significantly greater for Cav1.3 (PTXa mutant: $0.74 \pm 0.03$ a.u. (S.D. $=0.38$ a.u.) vs. PTXa control: $0.52 \pm 0.03$ a.u. (S.D. $=0.27$ a.u.); $n$ $=118, \mathrm{~N}=6$ for both genotypes, $p<0.00001$, Mann-Whitney-Wilcoxon test) and for PSD-95 (PTXa mutant: $0.37 \pm 0.02$ a.u. (S.D. $=0.20$ a.u.) vs. PTXa control: $0.28 \pm 0.01$ a.u. (S.D. $=0.15$ a.u.); $n=118, \mathrm{~N}=6$ for both conditions, $p=0.0009$ and Mann-Whitney-Wilcoxon test) (Figure $3 \mathrm{~A}$ and $\mathrm{B})$. Interestingly, we observed a strong positive correlation between the immunofluorescence intensities for Cav1.3 and its associated PSD-95 in PTXa control IHCs (Pearson coefficient of $0.74, \mathrm{p}<0.00001$ ), while this correlation was much weaker for PTXamutant IHCs (Pearson coefficient of $0.36, \mathrm{p}<0.001)$ ( $n=100$ spots, $\mathrm{N}=5$ for both conditions) (Figure 3C), indicating that the scaling of presynaptic densities (PDs) with PSD size is impaired upon PTXa expression. 
A
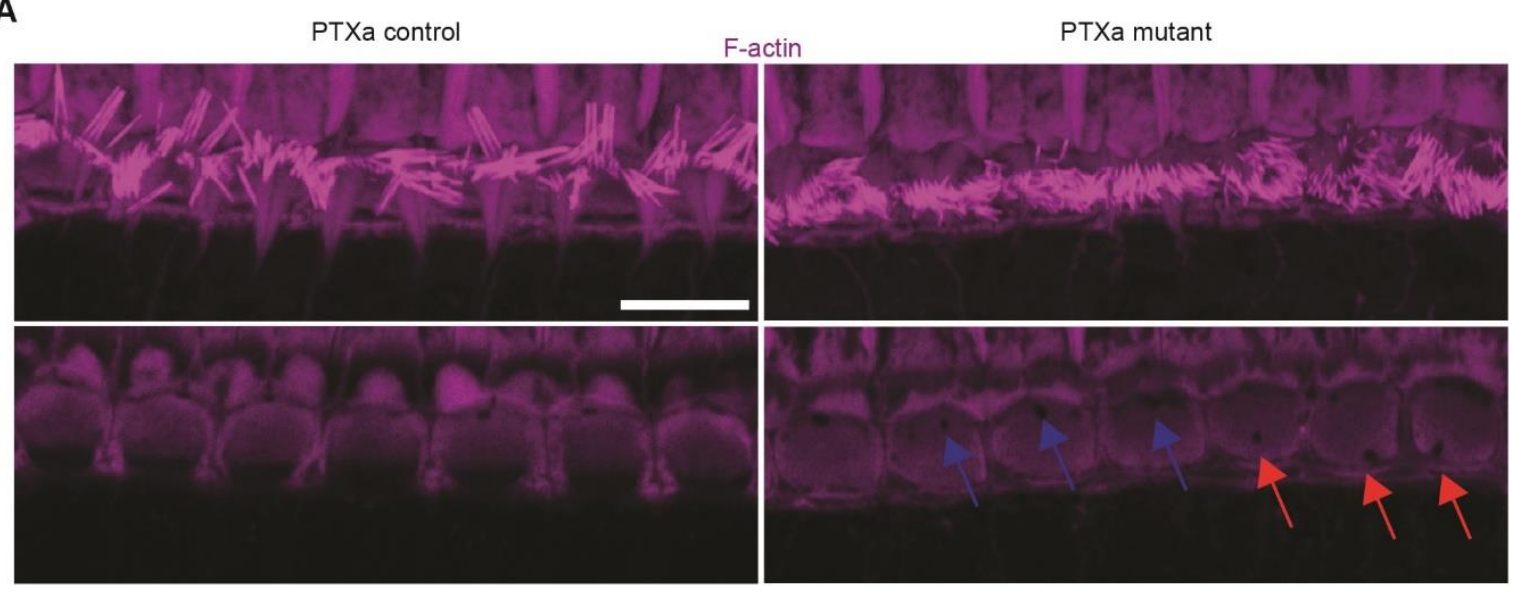

B

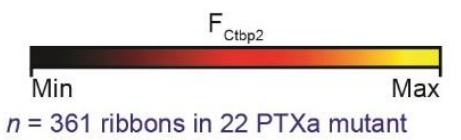

$n=359$ ribbons in 22

PTXa control IHCs

$n=361$ ribbons in 22 PTXa mutant non-inverted IHCs

$n=366$ ribbons in $22 \mathrm{PTX}$ mutant

$\mathrm{N}=5$

$\mathrm{N}=12$ $180^{\circ}$ inverted $\mathrm{IHCs}$

$\mathrm{N}=12$
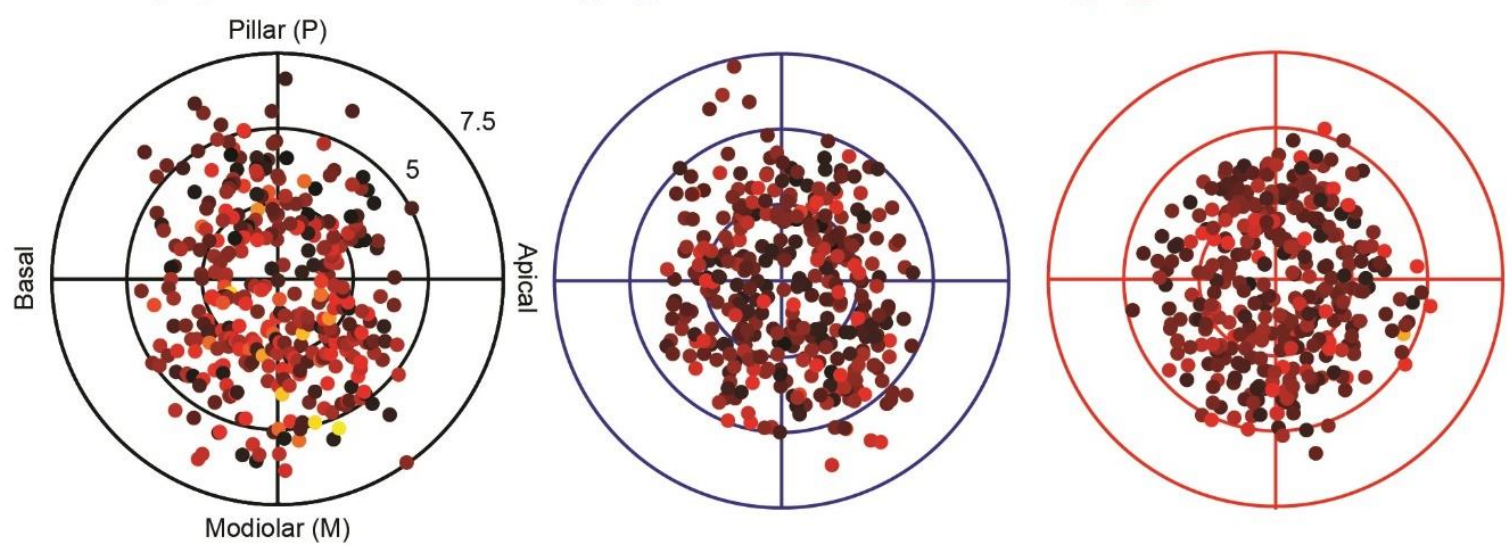

C

$* * * *$

n.s.
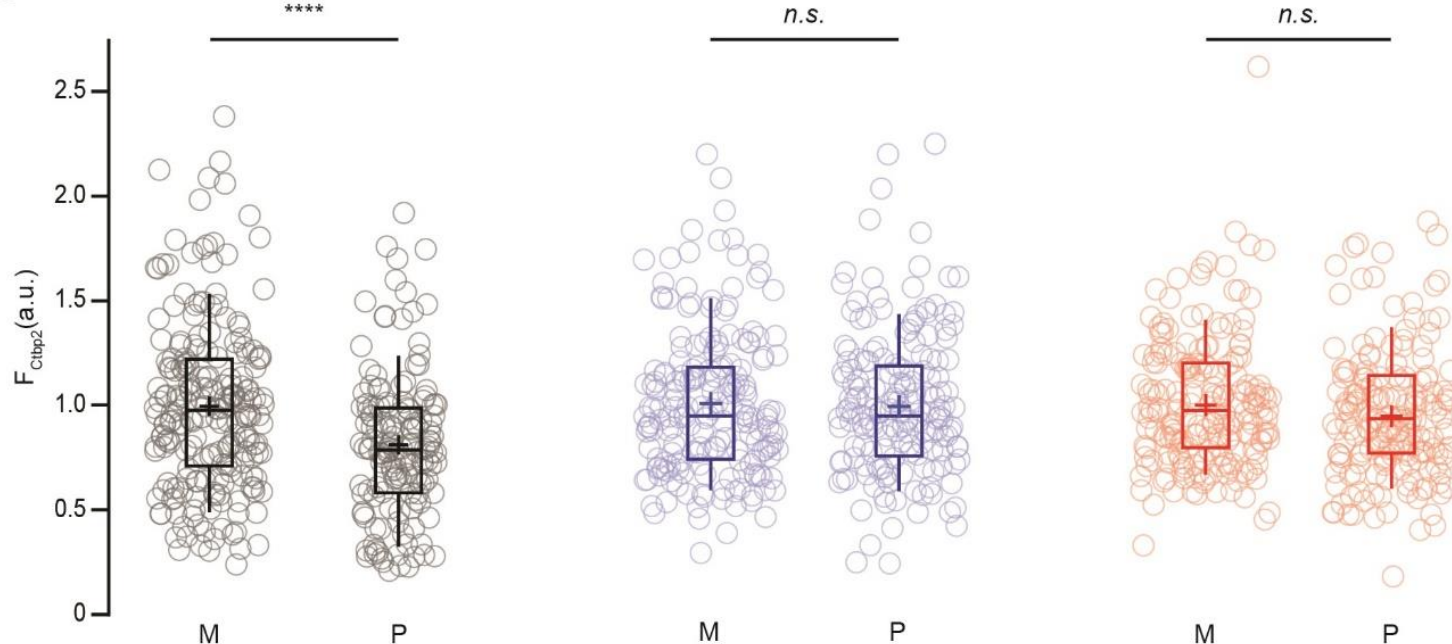


\section{Figure 1: Blocking of Gai signaling disrupts the modiolar-pillar gradient for ribbon size}

(A) Maximal projection (top row) and single confocal section (bottom row) of the IHC cuticular plates stained with phalloidin marking F-actin. The immunostainings exhibit the shortened and disorganized hair bundles in the PTXa mutant organ of Corti, as well as the misplaced fonticulus/cytoplasmic channel indicating the position of the basal body (blue arrows for PTXa mutant non-inverted cells, and red arrows for PTXa mutant $180^{\circ}$ inverted cells). Scale bar $=10 \mu \mathrm{m}$. (B) The polar charts (black for PTXa control, blue for PTXa mutant non-inverted cells, red for PTXa mutant $180^{\circ}$ inverted cells) display locations and intensities of immunofluorescently labelled CtBP2 marking the ribbons. Modiolar and pillar refer to facing to or from the ganglion in the modiolus, apical and basal refer to the tonotopic axis of the organ of Corti. The fluorescence intensity of each CtBP2 signal point is reflected by its color, with warmer, yellow tones indicating higher intensity, and cooler, darker tones indicating lower intensity. Each radial circle is $2.5 \mu \mathrm{m}$. (C) The IHCs from both PTXa control condition display a significant gradient with stronger ribbons in the modiolus (M) as compared to the pillar side (P) (PTXa control (black): modiolar: $n=211$ AZs vs Pillar: $n$ $=148 \mathrm{AZs}, p<0.00001$, Mann-Whitney-Wilcoxon test), while this gradient completely collapsed in the PTXa mutant IHCs, not depending on the kinocilium shift (PTXa mutant non-inverted (blue): modiolar: $n$ $=190$ AZs vs Pillar: $n=171$ AZs, $p=0.88$; PTXa mutant $180^{\circ}$ inverted (red): modiolar: $n=192$ AZs vs Pillar: $n=174$ AZs, $p=0.75$ Mann-Whitney-Wilcoxon test for both conditions). Box plots show 10, 25, 50,75 and 90th percentiles with individual data points overlaid, means are shown as crosses.

In order to analyze the spatial organization of synaptic $\mathrm{Ca}^{2+}$-channels, we performed 2color, 2D-STED imaging of Cav1.3 and PSD-95 immunofluorescence. Cav1.3 clusters were categorized according to their arrangements and number of structures (Jean et al., 2018; Neef et al., 2018) (Figure 3D, E). PTXa control and mutant IHCs exhibited comparable proportions of "point-like" clusters (9\% and $10 \%$, respectively; characterized by a Gaussian fitting ratio full width half maximum (FWHM) long axis/ FWHM short axis < 2, purple). While approximately $40 \%$ of the control AZs showed the typical "line-like" organization of Cav1.3 immunofluorescence (defined by a width inferior to $140 \mathrm{~nm}$, red), these structures were rare in mutant IHCs with a prevalence of only $8 \%$. However, the "fat line-like" clusters, defined as lines wider than 140nm (blue), had equivalent prevalence in both control (23\%) and mutant (27\%) conditions. Most strikingly, a difference was found in the proportion of complex clusters, defined by two or more Cav1.3 immunofluorescent structures at the synapse. Complex clusters were much more frequent in mutants (55\%) than in controls (26\%). Moreover, these complex arrangements exhibited up to two or three structures in the control AZs, while they could present up to five in the mutant ones (Figure 3D, E). Accordingly, the average number of Cav1.3-immunofluorescent spots was significantly higher at mutant synapses compared to control ones (PTXa mutant: 2.10 \pm 0.11 (S.D. $=1.26)$ vs. PTXa control: $1.34 \pm 0.06($ S.D.: $=0.61), n=120$ synapses, $\mathrm{N}=3$ for both conditions; $p<0.00001$, Mann-Whitney-Wilcoxon test), consistent with the stronger Cav1.3immunofluorescence intensity observed at the confocal level (Figure 3A, B). 

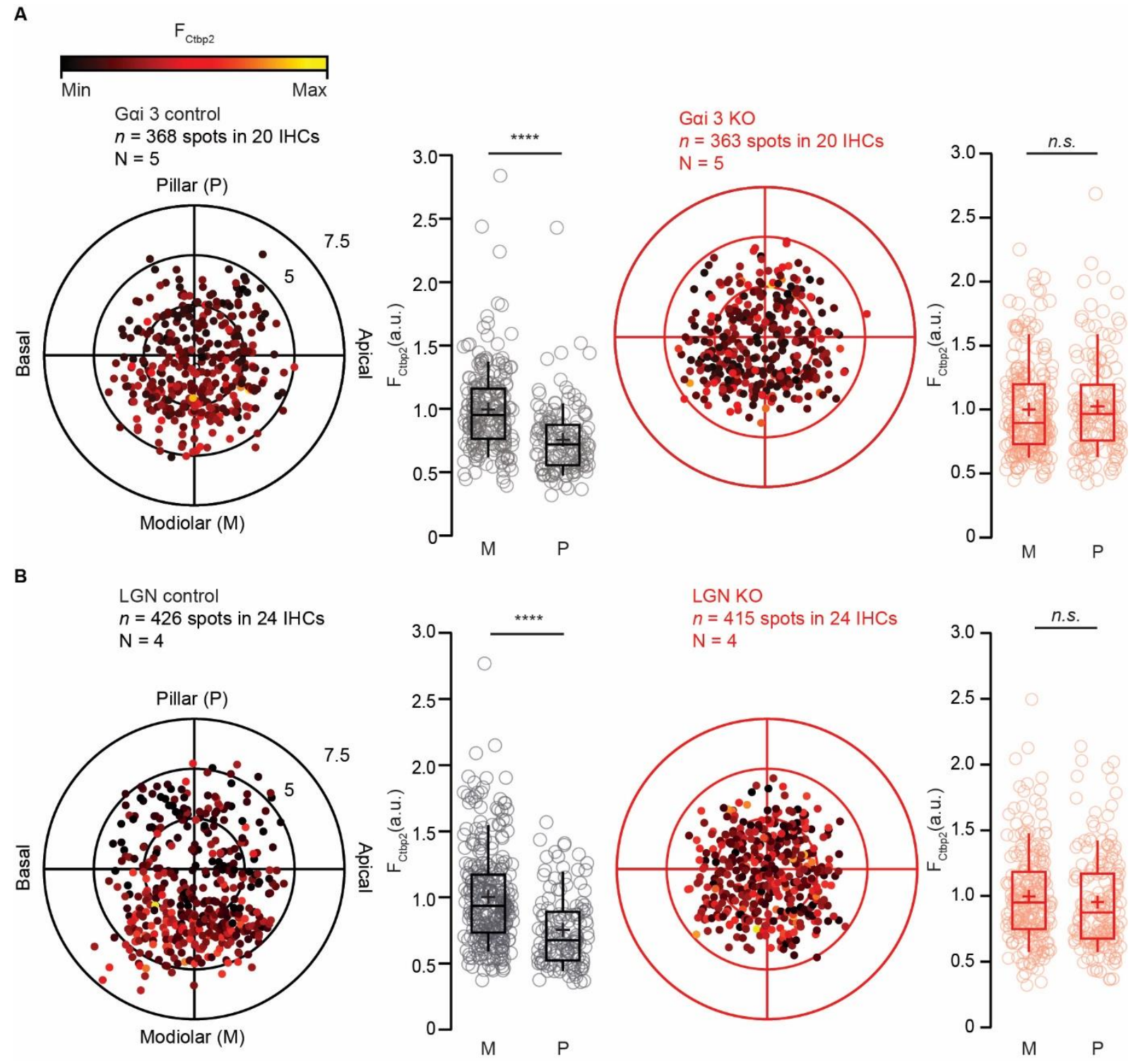

Figure 2: Deletion of Gai3 and LGN collapses the modiolar-pillar gradient for ribbon size

(A) The polar charts display locations and intensities of immunofluorescently labelled CtBP2 marking the ribbons. Modiolar and pillar refer to facing to or from the ganglion in the modiolus, apical and basal refer to the tonotopic axis of the organ of Corti. The fluorescence intensity of each CtBP2 signal point is reflected by its color, with warmer, yellow tones indicating higher intensity, and cooler, darker tones indicating lower intensity. Each radial circle is $2.5 \mu \mathrm{m}$. The spatial gradient for ribbon strength was lost upon Gai3 deletion (modiolar: $n=218$ AZs vs. pillar: $n=145$ AZs for $20 \mathrm{IHCs}, \mathrm{N}=5, p<0.00001$, Mann-WhitneyWilcoxon test) whereas it was preserved in the littermate controls (modiolar: $n=225$ AZs vs. pillar: $n=$ 143 AZs for $20 \mathrm{IHCs}, \mathrm{N}=5, p<0.00001$, Mann-Whitney-Wilcoxon test). Box plots show 10, 25, 50,75 and 90th percentiles with individual data points overlaid, each radial circle is $2.5 \mu \mathrm{m}$, means are shown as crosses as for (B). (B) The deletion of LGN collapsed the gradient of ribbon size (LGN KO: modiolar: $n=$ 231 AZs vs. pillar: $n=184$ AZs, $p=0.11$ vs. LGN Control: $n=298$ AZs vs. pillar: $n=128$ AZs, $p<$ $0.00001 ; 24 \mathrm{IHCs}, \mathrm{N}=4$, Mann-Whitney-Wilcoxon test for both conditions). 
This difference was most probably underestimated due to the low resolution of 2D-STED in the z-axis. Altogether, these data suggest that PTXa-expressing IHCs possess normally sized ribbons, whereas $\mathrm{Ca}_{v} 1.3 \mathrm{Ca}^{2+}$ channels are organized in larger and more complex clusters, juxtaposed to larger postsynaptic densities as compared to controls.

A

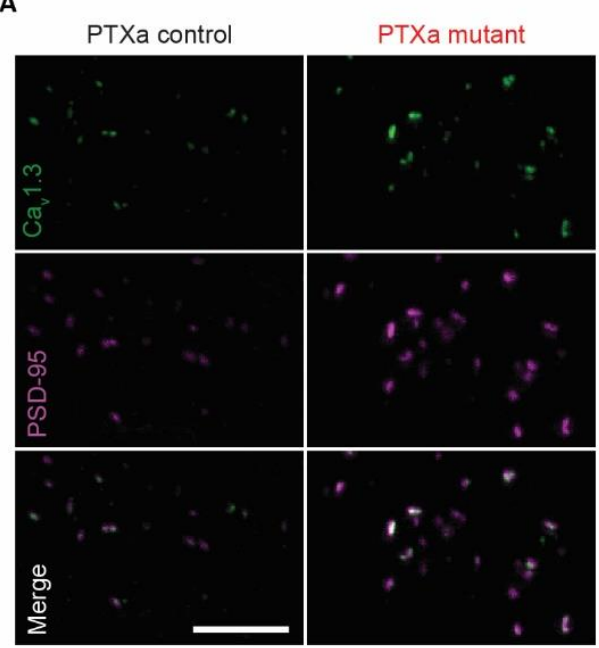

D

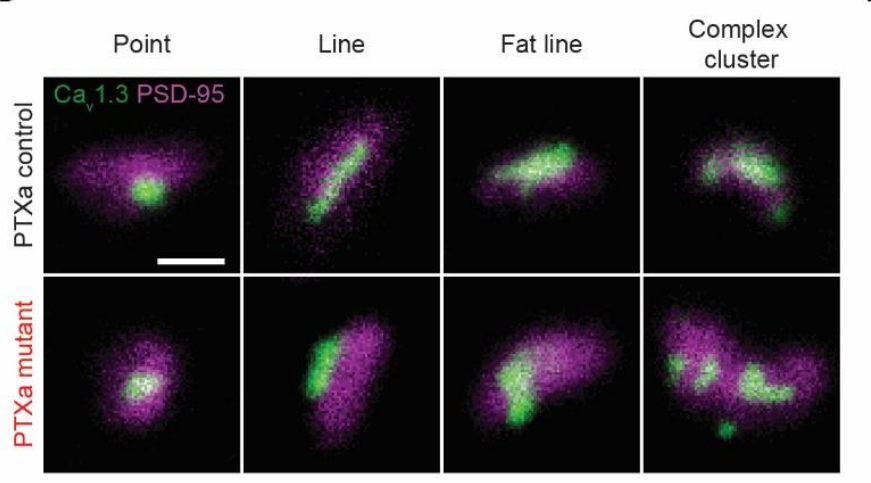

B

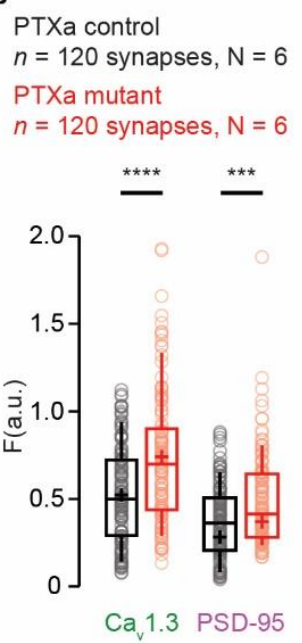

c
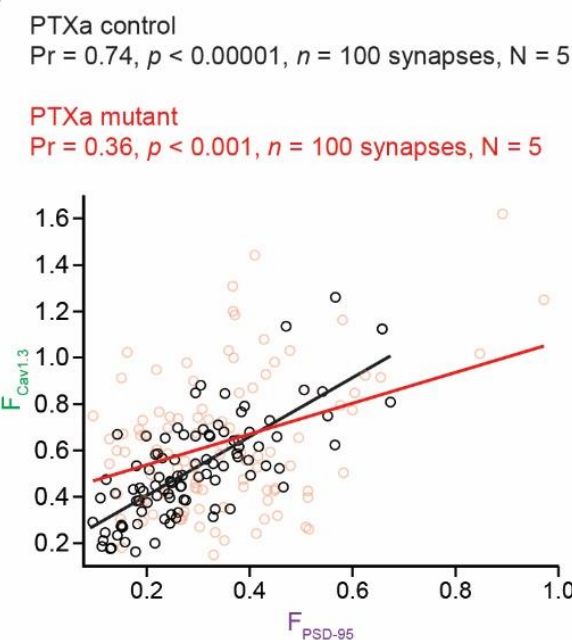

E

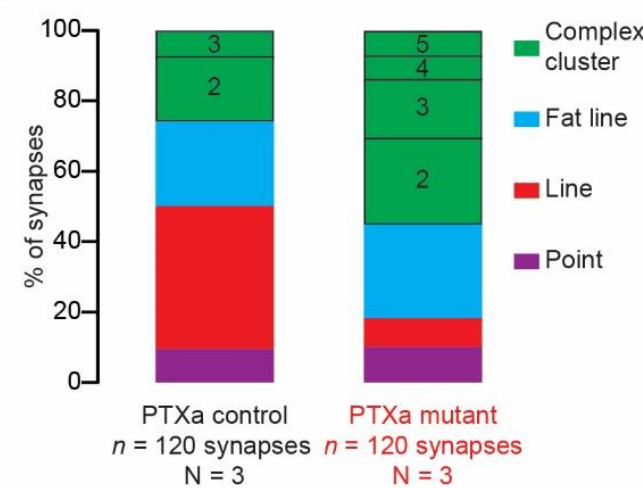

Figure 3: PTXa expressing IHC exhibit enlarged Cav1.3 channel and PSD-95 clusters

(A) Maximum intensity projections of confocal sections from organs of Corti immunolabeled for $\mathrm{Ca}_{\mathrm{v}} 1.3$ $\mathrm{Ca}^{2+}$-channels and PSD-95. Scale bar $=5 \mu \mathrm{m}$. (B) Mean intensities of synaptic Cav1.3 $\mathrm{Ca}^{2+}$-channel clusters and post-synaptic densities PSD-95 (estimated by the maximal amplitude of 2D Gaussian fits) were significantly increased in the PTXa mutant IHCs (red circles) as compared to PTXa control IHCs (black circles) ( $n=120$ synapses, $\mathrm{N}=6$ for both conditions, $p<0.00001$ and $=0.0009$ for Cav1.3 and PSD-95 spots respectively, Mann-Whitney-Wilcoxon test for both conditions). (C) Scatter plot displaying the intensities of a $\mathrm{Ca}_{\vee} 1.3$ spots against their associated PSD-95 spots. A positive correlation is found in the PTXa control condition (black circles, Pearson coefficient of 0.74, $\mathrm{p}<0.00001, n=100$ synapses, $\mathrm{N}=$ 5), while the correlation is much weaker in the PTXa mutant IHCs (red circles, Pearson coefficient of 0.36, $\mathrm{p}<0.001, n=100$ synapses, $\mathrm{N}=5$ ). (D) Representative examples of AZs immuno-labelled against $\mathrm{Ca} v 1.3$ $\mathrm{Ca}^{2+}$-channels and PSD-95, acquired with 2D- STED microscopy. AZs from both conditions exhibited point-like clusters (defined by a Gaussian fitting ratio L.A. / S.A. <2), some clusters formed lines (defined by a S.A. < 140nm), fat lines (defined by a S.A. > 140nm) and complex arrangements (defined by two or 
more structures). Scale bar $=500 \mathrm{~nm}$. (E) The $\mathrm{Ca}_{\mathrm{v}} 1.3 \mathrm{Ca}^{2+}$-channel clusters were categorized according to these arrangements. PTXa control and mutant IHCs exhibited similar proportions of point like clusters (9\% and $10 \%$, respectively, violet). Around $40 \%$ of the control AZs showed the typical line-like organization of Cav1.3 immunofluorescence, these structures were more sporadic in mutant IHCs (8\%, red). However, the fat line clusters (blue) had comparable prevalence in both PTXa control (23\%) and mutant (27\%) conditions. Strikingly, a difference was found for the proportion of complex clusters that were much more frequent in the PTXa mutant condition (55\% against $26 \%$ in the PTXa control IHCs). Additionally, these features exhibited up to three structures at the control AZs, while the mutant ones could present until five ( $n=120$ synapses, $\mathrm{N}=3$ for both conditions).

\section{Disrupting Gai signaling causes an increased amplitude, hyperpolarized activation and decreased exocytic efficiency of IHC Ca ${ }^{2+}$ influx}

To physiologically characterize the effect of disrupting Gai signaling with PTXa on Cav1.3 $\mathrm{Ca}^{2+}$ channels, we first recorded the whole-cell $\mathrm{Ca}^{2+}$-current in the ruptured-patch configuration $(5 \mathrm{mM}$ $\left[\mathrm{Ca}^{2+}\right]_{\mathrm{e}}$, Figure 4A). The amplitude of the $\mathrm{Ca}^{2+}$-influx (Figure $4 \mathrm{~A}_{\mathrm{i}}$ ) was substantially increased in PTXa-expressing IHCs (PTXa mutant: $-276 \pm 8$ pA (S.D. $=50$ pA), $n=40$ IHCs, N = 13 vs. PTXa control: $-191 \pm 6$ pA, S.D. $=38$ pA, $n=40$ IHCs, $\mathrm{N}=14 ; p<0.00001$, t-test), consistent with the more numerous Cav1.3 channels indicated by immunohistochemistry. Next, we analyzed the voltage-dependence of $\mathrm{Ca}^{2+}$-channel activation (Figure 4B), and found a significant hyperpolarizing shift of $-4 \mathrm{mV}$ of the voltage of half-maximal $\mathrm{Ca}^{2+}$-channel activation, $\mathrm{V}_{\mathrm{h}}$ (Figure 4B $\mathrm{B}_{\mathrm{i}}$ PTXa mutant: $-28.16 \pm 0.54 \mathrm{mV}$ (S.D. $\left.=3.41 \mathrm{mV}\right), n=40 \mathrm{IHCs}, \mathrm{N}=13$ vs. PTXa control: $24.34 \pm 0.46 \mathrm{mV}($ S.D. $=2.93 \mathrm{mV}), n=40 \mathrm{IHCs}, \mathrm{N}=14, p<0.00001$, t-test $)$. Moreover, we found a subtle but significant increase of the voltage-sensitivity of activation (reflected as a decrease of the slope factor $\mathrm{k}$ ) in mutant IHCs (PTXa mutant: $7.33 \pm 0.06 \mathrm{mV}$ (S.D. $=0.41 \mathrm{mV}$ ), $n=40$ IHCs, $\mathrm{N}=13$ vs. PTXa control: $7.72 \pm 0.07 \mathrm{mV}$ (S.D. $=0.42 \mathrm{mV}), n=40 \mathrm{IHCs}, \mathrm{N}=14$, $p<0.00001, \mathrm{t}$-test, Figure $\left.4 \mathrm{~B}_{\mathrm{ii}}\right)$.

In order to test the consequences of the $\mathrm{Cav} 1.3 \mathrm{Ca}^{2+}$ channel rearrangement in the PTXa mutant IHCs on exocytosis, we performed capacitance measurements $\left(\mathrm{C}_{\mathrm{m}}\right)$ in perforated patchclamp configuration (1.3 mM $\left[\mathrm{Ca}^{2+}\right]_{\mathrm{e}}$, Figure 4C-D). As expected, we found significantly increased $\mathrm{Ca}^{2+}$ charge $\left(\mathrm{Q}_{\mathrm{Ca}}\right.$, at $2 \mathrm{~ms}: p=0.002$, $\mathrm{Q}_{\mathrm{Ca}}$ at $5 \mathrm{~ms}: p=0.004, \mathrm{Q}_{\mathrm{Ca}}$ at $10 \mathrm{~ms}: p=0.004$, $\mathrm{Q}_{\mathrm{Ca}}$ at $20 \mathrm{~ms}: p=0.01, \mathrm{Q}_{\mathrm{Ca}}$ at $50 \mathrm{~ms}: p=0.01, n=8 \mathrm{IHCs}, \mathrm{N}=6$ in the PTXa mutant; $n=8 \mathrm{IHCs}$, $\mathrm{N}=5$ in the PTXa control; t-test, Figure 4C). The efficiency of $\mathrm{Ca}^{2+-i n f l u x}$ to drive exocytosis, quantified as the ratio of $\Delta \mathrm{C}_{\mathrm{m}}$ and $\mathrm{Q}_{\mathrm{Ca}}$, was reduced for 20 and $50 \mathrm{~ms}$ depolarization in PTXaexpressing IHCs as compared to control IHCs (20 ms: $p=0.013,50 \mathrm{~ms}: p=0.042 ; n=8 \mathrm{IHCs}, \mathrm{N}$ $=6$ in the PTXa mutant; $n=8$ IHCs, $\mathrm{N}=5$ in the PTXa control; t.test, Figure 4D). This might 
reflect an impaired spatial coupling of $\mathrm{Ca}^{2+}$-channels to vesicular release sites given the rearrangement of the $\mathrm{Cav} 1.3 \mathrm{Ca}^{2+}$ channels.

Next, we studied presynaptic $\mathrm{Ca}^{2+}$-signaling at individual AZs using spinning-disk confocal microscopy of IHCs loaded with the low-affinity $\mathrm{Ca}^{2+}$ indicator Fluo-4FF $(800 \mu \mathrm{M})$, the non-fluorescent chelator EGTA $(10 \mathrm{mM})$ and a TAMRA (tetramethylrhodamine)-conjugated CtBP2-binding peptide. Under these conditions the $\mathrm{Ca}^{2+}$ indicator fluorescence serves as a proxy of synaptic $\mathrm{Ca}^{2+}$ influx (Frank et al., 2009; Neef et al., 2018; Ohn et al., 2016). AZs were identified by spots of the CtBP2-binding peptide fluorescence where $\mathrm{Ca}^{2+-}$ signals (Fluo-4FF hotspots) commenced upon depolarization. We found an increased maximal rise of Fluo-4FF fluorescence, i.e. "synaptic $\mathrm{Ca}^{2+}$ influx" in PTXa expressing IHCs (PTXa mutant: $\Delta \mathrm{F} / \mathrm{F}_{\max }$ : $2.05 \pm 0.10$ (S.D. = 1.34), $n=175$ AZs in 20 IHCs, $\mathrm{N}=12$ vs. PTXa control: $1.59 \pm 0.08$ (S.D. $=1.10), n=175$ AZs in $20 \mathrm{IHCs}, \mathrm{N}=12, p=0.0002$, Mann-Whitney-Wilcoxon test; Figure 5A). This gain of synaptic $\mathrm{Ca}^{2+}$ signaling agrees with the observed increase in the number and intensity of synaptic Cav1.3 immunofluorescent clusters and with the enhanced $\mathrm{Ca}^{2+}$ influx at the whole-cell level. Moreover, as at the whole-cell level, we found a significant hyperpolarized shift $(-4 \mathrm{mV})$ of activation of the synaptic $\mathrm{Ca}^{2+}$ influx (PTXa mutant: $\mathrm{V}_{\mathrm{h}}:-28.77 \pm 0.71 \mathrm{mV}($ S.D. $=8.42 \mathrm{mV}), n=142 \mathrm{AZs}$ in 20 IHCs, $\mathrm{N}=12$ vs. PTXa control: $\mathrm{V}_{\mathrm{h}}:-24.11 \pm 0.57 \mathrm{mV}($ S.D. $=6.77 \mathrm{mV}), n=141 \mathrm{AZs}$ in $20 \mathrm{IHCs}$, $\mathrm{N}=12, p<0.0001$, Mann-Whitney-Wilcoxon test; Figure $5 \mathrm{~B}_{\mathrm{i}}$ ). However, the voltage sensitivity of activation was not significantly different for the synaptic $\mathrm{Ca}^{2+}$ influx in PTXa expressing IHCs likely due to higher variability at the single synapse level (PTXa mutant: k: 6.74 $\pm 0.17 \mathrm{mV}$ (S.D. $=1.99 \mathrm{mV}), n=142 \mathrm{AZs}$ in $20 \mathrm{IHCs}, \mathrm{N}=12$ vs. PTXa control: $6.81 \pm 0.18 \mathrm{mV}($ S.D. $=2.13 \mathrm{mV})$, $n=141$ AZs in 20 IHCs, $\mathrm{N}=12, p=0.55$, Mann-Whitney-Wilcoxon test; Figure $5 \mathrm{~B}_{\mathrm{ii}}$ ).

Finally, we studied the spatial extent of the synaptic $\mathrm{Ca}^{2+}$-signals by measuring the FWHM by fitting 2D Gaussian functions to the hotspots of $\mathrm{Ca}^{2+}$-indicator fluorescence. We found a greater spread of synaptic $\mathrm{Ca}^{2+}$-signals in PTXa-expressing IHCs (PTXa mutant: long axis (L.A.) $=1061 \pm 21 \mathrm{~nm}($ S.D. $=266 \mathrm{~nm})$, short axis $($ S.A. $)=779 \pm 18 \mathrm{~nm}($ S.D. $=228 \mathrm{~nm}) ; n=162 \mathrm{AZs}$ in 20 IHCs, $\mathrm{N}=12$ vs. PTXa control: L.A. $=909 \pm 20 \mathrm{~nm}($ S.D. $=252 \mathrm{~nm}) ;$ S.A. $=672 \pm 17 \mathrm{~nm}$ (S.D. $=218 \mathrm{~nm}), n=160$ AZs in $20 \mathrm{IHCs}, \mathrm{N}=12, p<0.0001$, Mann-Whitney-Wilcoxon test for both axis) (Figure 5C and D). This larger spread of the presynaptic $\mathrm{Ca}^{2+}$-signals is in agreement with the higher prevalence of multiple $\mathrm{Ca}^{2+}$-channel clusters observed at the AZs of PTXaexpressing IHCs. 


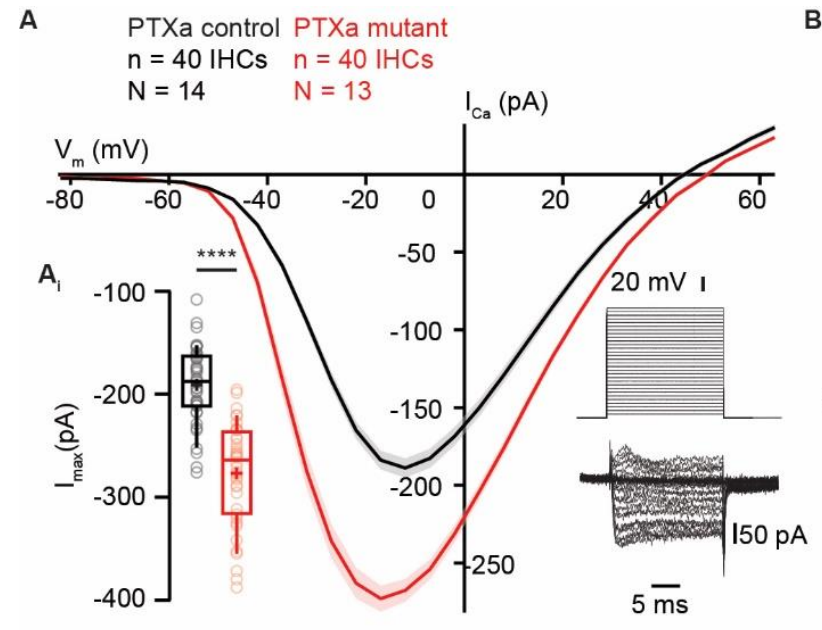

B
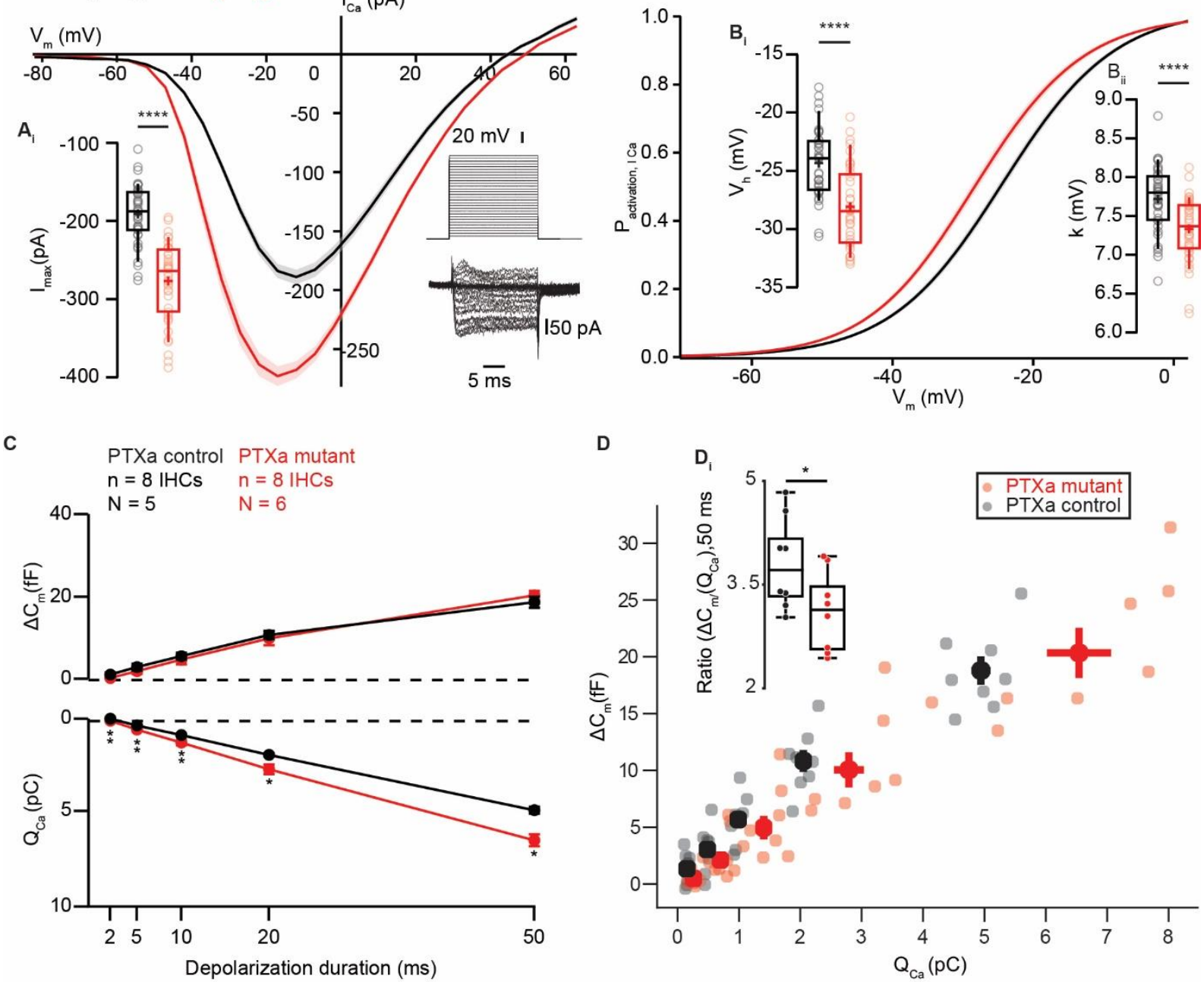

Figure 4: PTXa expressing IHCs show an increased and hyperpolarized $\mathrm{Ca}^{2+}$ influx at the whole-cell level, but with lower efficiency

(A) IV-relationship of the whole-cell $\mathrm{Ca}^{2+}$-current shows a significantly increased current amplitude in the PTXa mutant IHCs ( $n=40$ IHCs, $\mathrm{N}=13$ in the PTXa mutant; $n=40$ IHCs, $\mathrm{N}=14$ in the PTXa control; $p<0.0001$, t.test, $\mathbf{A}_{\mathbf{i}}$ ). The protocol, consisting of $20 \mathrm{~ms}$ steps of $5 \mathrm{mV}$ from -82 to $+63 \mathrm{mV}$, as well as the resulting currents of a representative control IHC, are shown in the bottom right. Mean (line) \pm S.E.M. (shaded areas) are displayed, the box plots show 10, 25, 50, 75 and 90th percentiles with individual data points overlaid and means are shown as crosses, as for (B). (B) Fractional activation of the whole-cell $\mathrm{Ca}^{2+}$ current derived from the IV-relationships $(\mathrm{A})$ was fitted to a Boltzmann function. $\left(\mathrm{B}_{\mathrm{i}}\right)$ Box plots of the voltage for half-maximal activation $\mathrm{V}_{\mathrm{h}}$ and $\mathrm{V}_{\mathrm{h}}$-estimates of individual IHCs show a significant hyperpolarized shift of the fractional activation $\left(\mathrm{V}_{\mathrm{h}}\right)$ of the $\mathrm{Ca}_{\mathrm{v}} 1.3 \mathrm{Ca}^{2+}$-channels in the PTXa mutant condition ( $n=40$ IHCs, $\mathrm{N}=13$ in the PTXa mutant; $n=40$ IHCs, $\mathrm{N}=14$ in the PTXa control; $p<0.0001$, t.test). $\left(\mathrm{B}_{\mathrm{ii}}\right)$ Box plots of the voltage-sensitivity or slope factor $\mathrm{k}$ and $\mathrm{k}$-estimates of individual IHCs illustrate decreased voltage sensitivity in the PTXa mutant condition ( $n=40 \mathrm{IHCs}, \mathrm{N}=13$ in the mutant; $n=40$ IHCs, $\mathrm{N}=14$ in the Ctrl; $p<0.0001$, t.test). (C) Mean exocytic $\Delta \mathrm{Cm}$ (top) and $\mathrm{Ca}^{2+}$ current integrals ( $\mathrm{Q}_{\mathrm{Ca}}$ ) (bottom) as a function of depolarization duration (mean \pm S.E.M., $n=8 \mathrm{IHCs}, \mathrm{N}=6$ in the PTXa mutant; $n=8 \mathrm{IHCs}, \mathrm{N}=5$ in the PTXa control; for $\mathrm{Q}_{\mathrm{Ca}}$ at $2 \mathrm{~ms}: \mathrm{p}=0.002$, $\mathrm{Q}_{\mathrm{Ca}}$ at $5 \mathrm{~ms}: \mathrm{p}=0.004$, $\mathrm{Q}_{\mathrm{Ca}}$ at 10 ms: $p=0.004, Q_{C a}$ at $20 \mathrm{~ms}: \mathrm{p}=0.01, \mathrm{Q}_{\mathrm{Ca}}$ at $50 \mathrm{~ms}: \mathrm{p}=0.01$, t.test). (D) Relation between exocytic $\Delta \mathrm{Cm}$ and $\mathrm{Q}_{\mathrm{Ca}}$ showed different efficiency of exocytosis between PTXa mutant and control IHCs. (Fi) Ratio of $\Delta \mathrm{Cm}$ 
and QCa from PTXa mutant and control IHCs (duration ( $n=8 \mathrm{IHCs}, \mathrm{N}=6$ in the PTXa mutant; $n=8$ IHCs, $\mathrm{N}=5$ in the PTXa control; for ratio at $20 \mathrm{~ms}$ : $\mathrm{p}=0.013$, QCa at $50 \mathrm{~ms}: \mathrm{p}=0.042$, t.test)

A

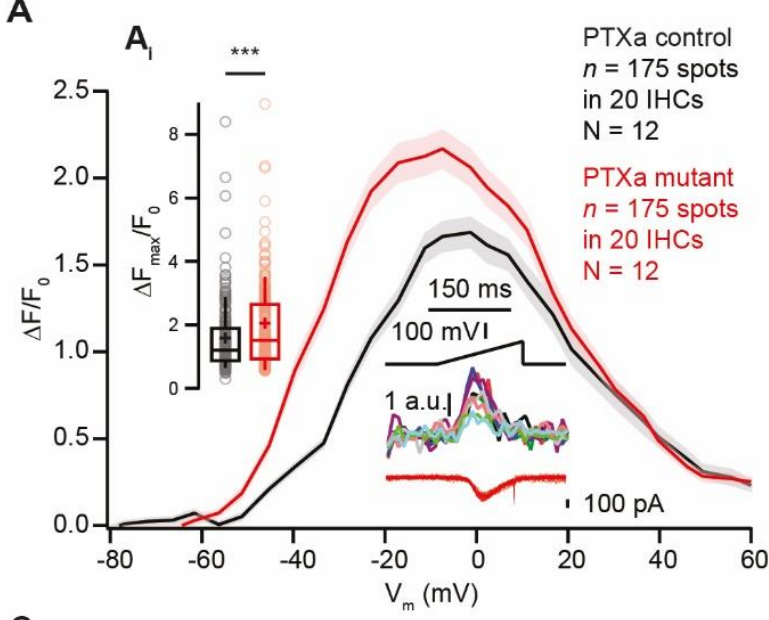

C

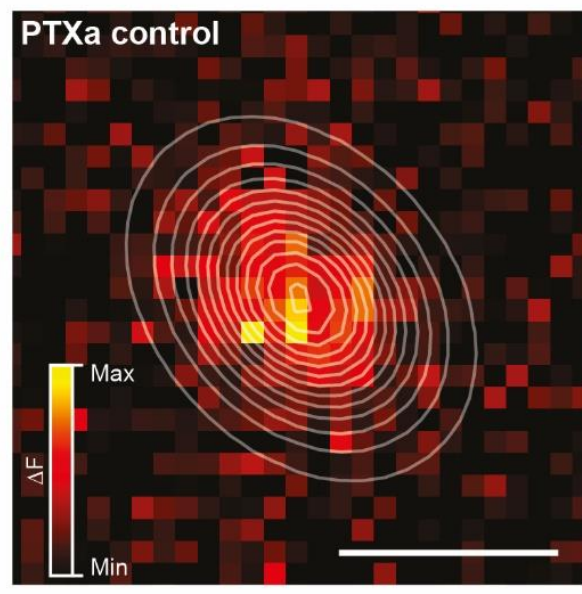

B

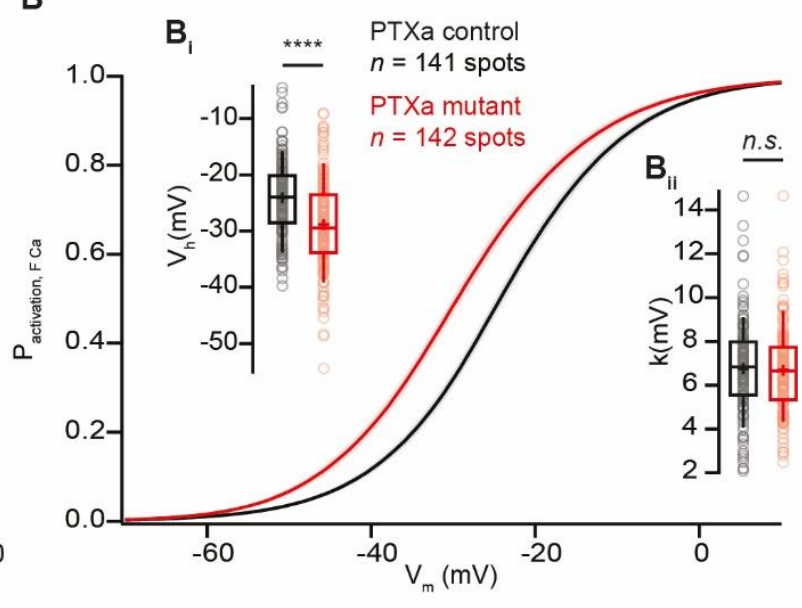

D $\begin{array}{ll}\text { PTXa control } & \text { PTXa mutant } \\ n=160 \text { spots } & n=162 \text { spots }\end{array}$

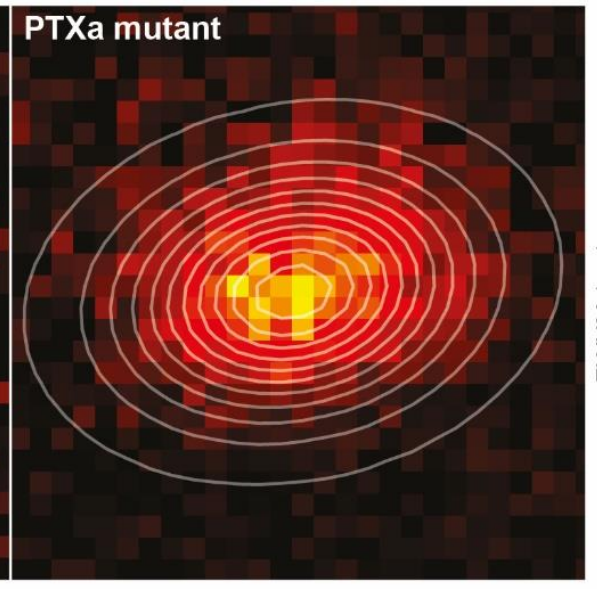

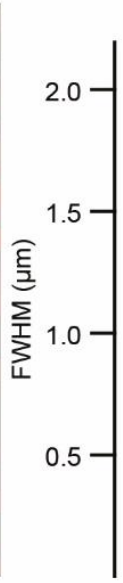
****

Figure 5: PTXa expressing IHCs possess an increased, more spread and hyperpolarized $\mathrm{Ca}^{2+}$ influx at the single synapse level

(A) FV-relationship ( $\triangle \mathrm{F} / \mathrm{F}_{0}$ vs. depolarization level in ramp): approximating the voltage-dependence of synaptic $\mathrm{Ca}^{2+}$-influx. Voltage-ramps from -87 to $+63 \mathrm{mV}$ during $150 \mathrm{~ms}$ were used to trigger synaptic hotspots of Fluo-4FF fluorescence and IHC Ca ${ }^{2+}$-influx (middle bottom, $10 \mathrm{AZs}$ in one exemplary IHC). $\left(\mathbf{A}_{\mathbf{i}}\right) \Delta \mathrm{Fmax} / \mathrm{F}_{0}$ was calculated by averaging 5 values at the $\mathrm{FV}$-peak and was significantly increased in the PTXa mutant condition ( $n=175$ AZs in 20 IHCs, $\mathrm{N}=12$ in the PTXa mutant; $n=175$ AZs in 20 IHCs, N $=12$ in the PTXa control; $p=0.0002$, Mann-Whitney-Wilcoxon test). Mean (line) \pm S.E.M. (shaded areas) are displayed, the box plots show 10, 25, 50, 75 and 90th percentiles with individual data points overlaid and means are shown as crosses, as for (B, D). (B) Fractional activation curves derived from fits to the FVrelationships $(C)$ were fitted to a Boltzmann function. $\left(\mathbf{B}_{\mathbf{i}}\right)$ The voltage for half-maximal activation $\mathrm{V}_{\mathrm{h}}$ was significantly hyperpolarized in the mutant condition ( $n=142$ AZs in 20 IHCs, $\mathrm{N}=12$ in the PTXa mutant; $\mathrm{n}=141 \mathrm{AZs}$ in $20 \mathrm{IHCs}, \mathrm{N}=12$ in the PTXa control; $p<0.0001$, Mann-Whitney-Wilcoxon test), while the voltage-sensitivity $\left(\mathbf{B}_{\text {ii) }}\right)$ was unchanged between both conditions $(n=142 \mathrm{AZs}$ in $20 \mathrm{IHCs}, \mathrm{N}=12$ in the PTXa mutant; $n=141$ AZs in 20 IHCs, $\mathrm{N}=12$ in the PTXa control; $p=0.55$, Mann-Whitney-Wilcoxon test). (C) Exemplary $\Delta$ F pictures of Fluo-4FF hotspots at PTXa control (left) and mutant (right) synapses 
fitted and overlaid by 2D-Gaussian functions to estimate spatial extent as full width at half maximum (FWHM) for the short axis (S.A.) and the long axis (L.A.). (D) AZs of PTXa mutant IHCs showed a greater spatial spread of the Fluo-4FF fluorescence change. FWHM calculated from the Gaussian fitting to the Fluo-4FF fluorescence hotspot was larger for both short and long axes in PTXa mutant IHCs $(n=162$ AZs for $20 \mathrm{IHCs}, \mathrm{N}=12)$ as compared to PTXa control ones $(n=160 \mathrm{AZs}$ for $20 \mathrm{IHCs}, \mathrm{N}=12)(p<0.0001$, Mann-Whitney-Wilcoxon test for both axis).

\section{The modiolar-pillar gradient of maximal synaptic $\mathrm{Ca}^{2+}$ influx is lost upon PTXa expression, but the pillar-modiolar gradient of the $\mathrm{Ca}^{2+}$ channel operating range is maintained}

By reconstructing the patched cells as cylindrical models, we then studied the positiondependence of AZ properties in live imaging experiments as previously described (Ohn et al., 2016). At P14-18 a tendency for a stronger maximal $\mathrm{Ca}^{2+}$ influx was found for modiolar AZs which also showed a significantly more depolarized activation of the $\mathrm{Ca}^{2+}$ channels. Performing this analysis on the synaptic $\mathrm{Ca}^{2+}$ influx of PTXa control and mutant IHCs at P21-26, as expected, we found a stronger $\Delta \mathrm{F} / \mathrm{F}_{\max }$ for modiolar than for pillar AZs in PTXa control IHCs (modiolar: $1.68 \pm 0.11$ (S.D. $=1.17), n=104$ AZs vs. pillar: $1.39 \pm 0.11$ (S.D. $=0.91), n=65$ AZs in 19 IHCs, $\mathrm{N}=11, p=0.033$, Mann-Whitney-Wilcoxon test). PTXa expression completely collapsed this gradient (modiolar: $1.96 \pm 0.13$ (S.D. $=1.32$ ),$n=98$ AZs vs. pillar: $1.94 \pm 0.17$ (S.D. $=1.31$ ), $n=57 \mathrm{AZs}$ in $18 \mathrm{IHCs}, \mathrm{N}=10, p=0.91$, Mann-Whitney-Wilcoxon test) (Figure 6A). The "winning" AZs, defined as the synapses exhibiting the strongest $\mathrm{Ca}^{2+}$ influx for each cell (highlighted in blue in the box plot and polar charts) were in great majority positioned on the modiolar side in PTXa control IHCs, as previously described (Ohn et al., 2016). In contrast, their localization seemed more random in PTXa-expressing IHCs. However, their respective contributions (ratio between mean $\Delta \mathrm{F} / \mathrm{F}_{\max }$ of winner / mean $\Delta \mathrm{F} / \mathrm{F}_{\max }$ of the rest of the $\mathrm{AZs}$ ) for a given cell did not differ significantly between the genotypes (PTXa mutant: $2.49 \pm 0.18$ (S.D. $=$ 0.78 ) vs. PTXa control: $2.27 \pm 0.24$ (S.D. $=1.08), p=0.46$, t.test). In contrast to the $\Delta \mathrm{F} / \mathrm{F}_{\max }$ gradient, the pillar-modiolar gradient for the $\mathrm{V}_{\mathrm{h}}$ of $\mathrm{Ca}^{2+}$ channel activation was maintained in PTXa expressing IHCs (PTXa mutant: modiolar : $-26.35 \pm 0.83 \mathrm{mV}($ S.D. $=7.50 \mathrm{mV}), n=80$ AZs vs. pillar: $-30.64 \pm 1.47 \mathrm{mV}$ (S.D. $=9.57 \mathrm{mV}$ ), $n=42 \mathrm{AZs}$ in $18 \mathrm{IHCs}, \mathrm{N}=10 ; p=0.013 \mathrm{vs}$. PTXa control: modiolar: $-23.19 \pm 0.74 \mathrm{mV}$ (S.D. $=6.69 \mathrm{mV}), n=81$ AZs vs. pillar: $-25.70 \pm 0.96$ $\mathrm{mV},(\mathrm{S} . \mathrm{D} .=6.96 \mathrm{mV}), n=53 \mathrm{AZs}$ in $19 \mathrm{IHCs}, \mathrm{N}=11 ; p=0.039$, t-test for both conditions) (Figure 6B). 
A
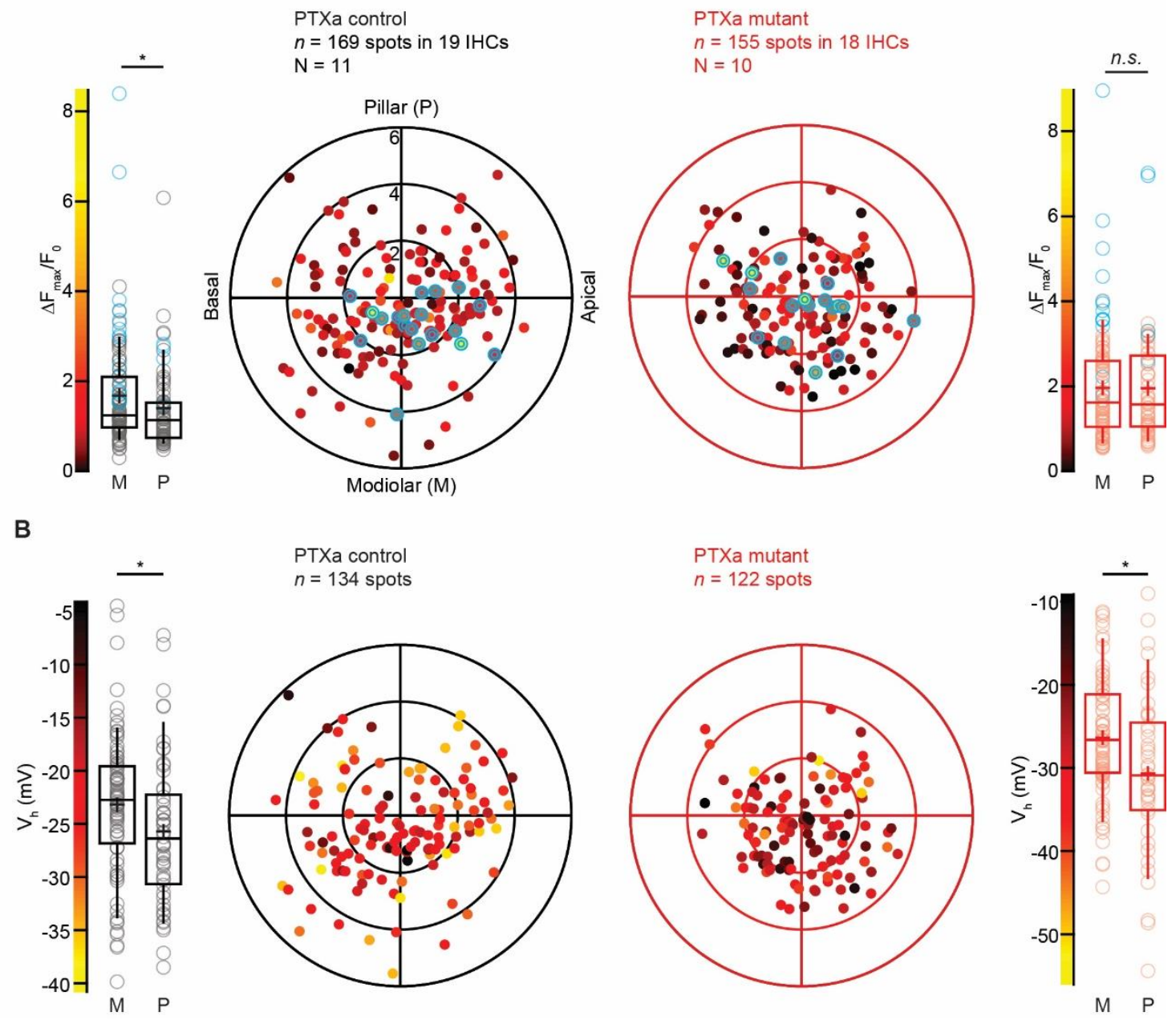

Figure 6: The modiolar-pillar gradient for synaptic $\mathrm{Ca}^{2+}$ influx strength is collapsed upon PTXa expression, but the one for voltage dependence is preserved

(A) The polar charts display intensities of maximal AZ Ca ${ }^{2+}$-influx $\left(\Delta \mathrm{F}_{\max } / \mathrm{F}_{0}\right)$ as a function of $\mathrm{AZ}$ positions in live-imaging experiments. Modiolar and pillar refer to facing to or from the ganglion in the modiolus; apical and basal refer to the tonotopic axis of the organ of Corti, as for (B). Box plots of the $\Delta \mathrm{F}_{\max } / \mathrm{F}_{0}$ and $\Delta \mathrm{F}_{\max } / \mathrm{F}_{0}$-estimates of individual IHCs show that the PTXa control condition exhibit significantly stronger $\mathrm{Ca}^{2+}$ hotspots in the modiolar side as compared to the pillar side (PTXa control: modiolar: $n=104$ AZs; pillar: $n=65 \mathrm{AZs}, p=0.033$, Mann-Whitney-Wilcoxon test), whereas this gradient completely collapse in the PTXa mutant (PTXa mutant: modiolar: $n=98$ AZs; pillar: $n=57$ AZs, $p=0.91$, Mann-WhitneyWilcoxon test). The strongest AZs from each cell are highlighted in blue in both polar charts and box plots. Data were pooled from 19 and 18 IHCs in PTXa control and mutant conditions respectively, box plots show 10, 25, 50, 75 and 90th percentiles with individual data points overlaid; each radial circle is $2 \mu \mathrm{m}$, means are shown as crosses, as for (B). (B) The polar charts display voltages for half-maximal activation $\mathrm{V}_{\mathrm{h}}$ as a function of $\mathrm{AZ}$ positions in live-imaging experiments. Box plots of the voltage for half-maximal activation $\mathrm{V}_{\mathrm{h}}$ and $\mathrm{V}_{\mathrm{h}}$-estimates of individual IHCs show a significant hyperpolarized shift of the fractional activation of the $\mathrm{Ca}_{\mathrm{v}} 1.3$ channels in the pillar side as compared to modiolar side in both control and mutant conditions (PTXa mutant: modiolar: $n=80$ AZs; pillar: $n=42$ AZs, $p=0.013$; PTXa control: modiolar: $n$ $=81 \mathrm{AZs}$; pillar: $n=53 \mathrm{AZs}, p=0.039$; t-test for both conditions). 


\section{Discussion}

The auditory system processes sound pressures ranging over six orders of magnitude. SGNs with different and complementary firing properties teamwork to encode this wide dynamic range of audible sounds. Despite progress in defining mechanisms, the functional diversity of SGNs remains largely enigmatic. Defining where the primary mechanism resides and how it is established and maintained are active fields of research. Here, we investigated whether Gai signaling, known to influence cell-intrinsic planar polarity at the apex of the HCs, also affects the presynaptic heterogeneity at the base of IHCs. Interestingly, disrupting Gai signaling via pertussis toxin expression in IHCs collapsed the modiolar-pillar gradients of ribbon size and maximal synaptic $\mathrm{Ca}^{2+}$ influx, while reorganizing and enlarging the synapses. Indeed, we found larger and more complex $\mathrm{Ca}^{2+}$ channel clusters by immunofluorescence microscopy, which was corroborated by the observation of increased $\mathrm{Ca}^{2+}$ influx at the whole-cell and single synapse levels. Interestingly, this modified $\mathrm{Ca}^{2+}$ influx showed lower efficiency in driving exocytosis. Since deletion of Gai3 and its direct binding partner LGN also disrupted the modiolar-pillar gradient of ribbon size, we propose a contribution of Gai3/LGN signaling in establishing the positiondependent properties of IHC AZs.

\section{A role for Gai signaling in setting up the spatial gradient of AZ properties in IHCs}

Although it is well-established that PTXa specifically ADP-ribosylates and impairs Gai function (Locht et al., 2011), it is difficult to entirely rule out unwanted consequences of PTXa expression in IHCs. Multiple observations argue against PTXa generally impairing IHC maturation of function, however. i) The targeted inactivation of Gai3 or its binding partner LGN collapsed the ribbon synapse size gradient regardless of IHC orientation, suggesting that the similar collapse upon PTXa reflects defective Gai3-LGN signaling and defective cell polarization. ii) The enlarged synaptic calcium influx observed upon PTXa argues against decreased IHC fitness or impaired IHC activity. iii) One marker of IHC maturity is the clustering of large-conductance $\mathrm{Ca}^{2+}$ activated $\mathrm{K}^{+}(\mathrm{BK})$ channels at the IHC neck, which are lacking in immature IHCs (Pyott, 2004; Sendin et $a l ., 2007)$. Although apparently reduced in number, these channels were present in Gai3 mutants (Figure supplement 3A), suggesting that the lack of modiolar-pillar gradient for ribbon size in Gai3 and PTXa mutants does not simply reflect an immature state. Supportive evidence also includes the report of a significant gradient before hearing onset (Liberman and Liberman, 2016). In contrast, PTXa-expressing IHCs showed variable BK signals from IHC to IHC ranging from 
normal clustering to no signal (Figure supplement 3B). Along with the reported absence of BK signals in Gai2; Gai3 combined mutants (Beer-Hammer et al., 2018), Gai signaling could thus be required for IHC maturation independently from its role in diversifying ribbon synapse properties. While the increased whole-cell $\mathrm{Ca}^{2+}$ current per se could be consistent with impaired IHC maturation (e.g. (Sendin et al., 2007), further arguments against a major maturational deficit include: (i) qualitatively comparable amount of extra-synaptic Cav1.3 $\mathrm{Ca}^{2+}$ channels at the confocal level, (ii) well-defined PSDs at the confocal and STED levels, in contrast to the several small PSDs per contact found at earlier stages (Wong et al., 2014), (iii) same ribbon intensity and number, while more and smaller ribbons were found in immature IHCs, and finally (iv) stronger synaptic $\mathrm{Ca}^{2+}$ influx, while weaker $\mathrm{Ca}^{2+}$ influx per $\mathrm{AZ}$ were found before hearing onset (Wong et $a l ., 2014)$. In conclusion, our results strongly suggest that Gai-LGN signaling is directly regulating the modiolar-pillar gradient of synapse properties.

Considering other possible confounding mechanisms, it is worth noting that stunted and disorganized stereocilia affecting hair bundle function and thus sound encoding are unlikely to alter synapses, as we did not find obvious changes in position-dependent morphological synapse properties in Myol $5^{\text {sh } 2}$ mutants. Nonetheless, some aspects of the synaptic phenotype observed in the PTXa mutant IHCs are reminiscent of the USH1C deaf-circler mutant mice, where absence of the harmonin protein induces severe hair bundle defects. There, too, an increased amplitude and hyperpolarized activation of synaptic $\mathrm{Ca}^{2+}$ influx was observed, but no evidence for an immaturity phenotype was found (Ohn et al., 2016). A gain of synaptic function through increased number and more hyperpolarized activation of $\mathrm{Ca}^{2+}$ channels could be a mechanism to increase spontaneous SGN firing in order to compensate for the lack of sound-evoked firing. Hence, the potentiated $\mathrm{Ca}^{2+}$ influx could be secondary to a deficit of mechanotransduction.

\section{Other candidates potentially affecting the spatial arrangement of $\mathrm{AZ}$ calcium influx properties}

Using single cell RNA sequencing, recent studies (Petitpré et al., 2018; Shrestha et al., 2018; Sun et al., 2018) characterized three distinct subpopulations of type I SGNs in the mouse. Interestingly, these transcriptome based subgroups expressed distinct complements of transcription factors but also ion channels, receptors and synaptic proteins. Moreover, as these different subpopulations were shown to differentially target the IHC basolateral periphery, their profiling offered potential post synaptic determinants that could account for heterogeneous firing properties of the SGNs, 
and perhaps also a instructive influence on spatial AZ organization in IHCs. By reconstructing the patch-clamped cells we found that blocking Gai signaling abolishes the modiolar-pillar gradient for the maximal strength of synaptic $\mathrm{Ca}^{2+}$ influx, but preserves the pillar-modiolar gradient for the voltage-dependence of $\mathrm{Ca}^{2+}$ channel activation. This finding might indicate that distinct mechanisms govern the spatial distribution of the number of $\mathrm{Ca}^{2+}$ channels per $\mathrm{AZ}$ and their voltage-dependence of activation. This observation of unchanged spatial gradient of voltagedependence of activation would be consistent with observations in IHCs of Gipc3 KO mice, where the gradient for the strength of synaptic $\mathrm{Ca}^{2+}$ influx was reversed while the pillar-modiolar one for voltage-dependence of activation was maintained (Ohn et al., 2016). Mutation of the Gipc $3^{343 \mathrm{~A}}$ allele disrupts the hair bundle structure and leads to progressive sensorineural hearing loss (Charizopoulou et al., 2011), while Gipc1 was shown to interact with the core PCP protein Vang12, and its disruption leads to maturation defects affecting hair bundle orientation and integrity (Giese et al., 2012). Interestingly, an interaction between Gipc1 and Tyrosine kinase receptor TrkA was described in culture, proposing Gipc1 as a link between TrkA and G protein signaling (Lou et al., 2001). The voltage-dependence of activation can be determined by the precise splice Cav1.3 variant (Bock et al., 2011; Ohn et al., 2016; Tan et al., 2011), the subunit composition (Cui et al., 2007) and interacting proteins present at the AZ (Gregory et al., 2013; Jean et al., 2018; Jung et al., 2015; Ohn et al., 2016).

Several studies in the neuromast HCs of larval zebrafish including modified expression of RIBEYE (Sheets et al., 2011, 2017) and frame shift mutations (Lv et al., 2016), as well as Credeletion of RIBEYE in the mouse cochlea and retina (Becker et al., 2018; Jean et al., 2018; Maxeiner et al., 2016) have shown a relationship between the ribbon and the $\mathrm{Ca}^{2+}$ channels cluster organization, but a direct interaction of RIBEYE and the $\mathrm{Ca}^{2+}$ channel complex has not yet been reported. Aside from RIBEYE, Bassoon has been shown to promote $\mathrm{Ca}^{2+}$-channel tethering at the AZ (Frank et al., 2010) likely via interaction with RIM-binding protein (Davydova et al., 2014). RIM-binding protein was reported to interact with Cav1.3 $\mathrm{Ca}^{2+}$-channels (Hibino et al., 2002) and is necessary to establish a normal $\mathrm{Ca}^{2+}$-channel complement at the IHC AZ (Krinner et al., 2017). Moreover, RIM2 $\alpha$ and $\beta$ have been reported to promote the clustering of these channels at the synapse (Jung et al., 2015), and RIM2 and RIM3 were shown to directly interact with the poreforming subunit of Cav1.3 $\mathrm{Ca}^{2+}$ channel (Picher et al., 2017). It will be of interest to explore possible interactions determining whether the trafficking of these different AZ proteins are governed by the Gai3-LGN complex. Future experiments are required to investigate on the 
individual contributions of the Gai proteins, their interacting partners and receptors as well as other planar polarity protagonists. In addition, emphasis should be put on ultrastructural studies by electron microscopy to further characterize the spatial reorganization of the synapses, as well as single unit recordings to assess the effect on the SGN firing rate diversity.

\section{Materials and methods}

\section{Mice}

The PTXa KI and LGN KO mice have been previously described (Tarchini et al., 2013, 2016). The Gail and Gai3 mutants were obtained from the Jackson Laboratory (JR\#24525), as was Myo15 $5^{\text {sh2 }}$ (JR\#109). All experiments complied with national animal care guidelines and were approved by the University of Göttingen Board for Animal Welfare and the Animal Welfare Office of the State of Lower Saxony and by the Animal Care and Use Committee of The Jackson Laboratory.

\section{Patch-clamp recordings}

The apical 2/3 turns of organs of Corti from P21-26 old mice were freshly dissected in HEPES Hank's solution containing (in $\mathrm{mM}$ ): $5.36 \mathrm{KCl}, 141.7 \mathrm{NaCl}, 10 \mathrm{HEPES}, 0.5 \mathrm{MgSO}_{4}-7 \mathrm{H}_{2} \mathrm{O}, 1$ $\mathrm{MgCl}_{2}-6 \mathrm{H}_{2} \mathrm{O}, 1 \mathrm{mg} / \mathrm{ml} \mathrm{D}$-glucose, and $0.5 \mathrm{mg} / \mathrm{ml} \mathrm{L-glutamine} \mathrm{(pH} \mathrm{7.2,} \mathrm{300} \mathrm{mOsm).} \mathrm{The} \mathrm{native}$ morphologies and positions of the IHCs within the organ of Corti were maintained as much as possible by accessing them from the modiolus. Patch pipettes were made from GB150F-8P or GB150-8P borosilicate glass capillaries for whole cell and perforated patch-clamp recordings, respectively (Science Products, Hofheim, Germany). All experiments were conducted at room temperature $\left(20-25^{\circ} \mathrm{C}\right)$. For whole cell recordings, the patch pipette solution contained (in $\left.\mathrm{mM}\right)$ : 111 L-glutamate, $1 \mathrm{MgCl}_{2}, 1 \mathrm{CaCl}_{2}$, 10 EGTA, 13 TEA-Cl, 20 HEPES, 4 Mg-ATP, 0.3 Na-GTP and 1 L-Glutathione ( $\mathrm{pH} \mathrm{7.3,} \mathrm{290} \mathrm{mOsm).} \mathrm{Perforated} \mathrm{patch-clamp} \mathrm{experiments} \mathrm{were} \mathrm{done} \mathrm{as}$ described previously (Moser and Beutner, 2000). The patch solution contained (in mM): 130 Csgluconate, 10 TEA-Cl, 10 4-AP, $10 \mathrm{HEPES}, 1 \mathrm{MgCl}_{2}$, as well as $300 \mathrm{mg} / \mathrm{ml}$ amphotericin B (pH 7.17, $290 \mathrm{mOsm}$ ). The extracellular solution contained the following (in $\mathrm{mM}$ ): $2.8 \mathrm{KCl}, 102$ $\mathrm{NaCl}, 10 \mathrm{HEPES}, 1 \mathrm{CsCl}_{2}, 1 \mathrm{MgCl}_{2}$, 35 TEA-Cl, $2 \mathrm{mg} / \mathrm{ml}$ D-Glucose and either $5 \mathrm{CaCl}_{2}$ or 1.3 $\mathrm{CaCl}_{2}$ for whole cell and perforated patch-clamp configuration, respectively ( $\mathrm{pH} 7.2, \sim 300$ mOsm). An EPC-10 USB amplifier (HEKA, Lambrecht/Pfalz, Germany) controlled by PatchMaster software (HEKA) was used for the acquisition. IHCs were held at $-87 \mathrm{mV}$ after liquid 
junction potential correction. All voltages were corrected offline for liquid junction potential (-17 $\mathrm{mV}$ ) and voltage-drops across the series resistance $\left(\mathrm{R}_{\mathrm{s}}\right)$. For whole-cell recordings, recordings were discarded when the leak current exceeded $-50 \mathrm{pA}, \mathrm{R}_{\mathrm{s}}$ exceeded $15 \mathrm{M} \Omega$ within 4 min after break-in, or $\mathrm{Ca}^{2+}$-current rundown exceeded $25 \%$. The membrane capacitance changes (DCm) were used to study exocytosis using the Lindau-Neher technique (Lindau and Neher, 1988). For capacitance measurements, traces with the leak current lower than $-30 \mathrm{pA}$ and $\mathrm{R}_{\mathrm{s}}$ lower than 30 $\mathrm{M} \Omega$ were used for further analysis. IHCs were step depolarized from holding potential to $-17 \mathrm{mV}$ for durations of $2 \mathrm{~ms}$ to $50 \mathrm{~ms}$.

\section{Spinning disk microscopy and live confocal calcium imaging}

$\mathrm{Ca}^{2+}$-imaging was performed with a spinning disk confocal scanner (CSU22, Yokogawa, Germany) mounted on an upright microscope (Axio Examiner, Zeiss, Germany) with 63x, 1.0 NA objective (W Plan-Apochromat, Zeiss). The spinning disk was set to $2000 \mathrm{rpm}$ to synchronize with the $100 \mathrm{~Hz}$ acquisition rate of the camera to avoid the uneven illumination. Images were acquired by a scientific CMOS camera (Neo, Andor, Germany), with a pixel size of $103 \mathrm{~nm}$. To visualize the $\mathrm{Ca}^{2+}$-hotspots and the ribbons, the low affinity $\mathrm{Ca}^{2+}$-indicator Fluo-4FF penta- $\mathrm{K}^{+}$salt (0.8 mM, Life Techologies, Germany) and the TAMRA-conjugated CtBP2/RIBEYE-binding dimer peptide (10 $\mu \mathrm{M}$, Biosynthan, Germany) were added to the intracellular solution just before experiment. The $\mathrm{Ca}^{2+}$-indicator Fluo-4FF was excited by the $491 \mathrm{~nm}$ diode-pump solid-state (DPSS) laser (Calypso, Cobolt AB, Solna, Sweden), and the red fluorescence from TAMRA was excited by the $561 \mathrm{~nm}$ DPSS laser (Jive, Cobolt AB). Using a piezo positioner for the objective (Piezosystem, Germany), a scan of the entire cell was performed 4 min after breaking into the cell, taking sections each $0.5 \mu \mathrm{m}$ at an exposure time of $0.5 \mathrm{~s}$ in the red (TAMRA-peptide) channel from the bottom to the top of the cell. In order to study the voltage-dependence of $\mathrm{Ca}^{2+}$-indicator fluorescence increments at the synapses, the confocal scans were acquired every $0.5 \mu \mathrm{m}$ from the bottom-most to the top-most ribbon. $\mathrm{Ca}^{2+}$-currents were evoked by applying a voltage ramp stimulus from -87 to $+63 \mathrm{mV}$ during $150 \mathrm{~ms}(1 \mathrm{mV} / \mathrm{ms})$ in each focal plane. Simultaneously, fluorescence measurements were made in the green channel (Fluo-4FF) with a frame rate of 100 Hz. In order to overcome the limitations of the frame rate and increase the voltage resolution of the fluorescent signal acquired, the voltage ramp protocol was applied twice, once shifted by $5 \mathrm{~ms}$ such that for any given frame during the second ramp the voltage was shifted by $5 \mathrm{mV}$ compared to the first stimulus. The interval between 2 sequential sections was $2 \mathrm{~s}$ to eliminate the effect of calcium dependent inactivation. Alternating planes were acquired to avoid photobleaching 
encountered with the consecutive plane acquisitions.

\section{Immunohistochemistry, confocal and high resolution STED imaging}

The samples were fixed in formaldehyde (4\%, $10 \mathrm{~min}$ to $1 \mathrm{~h}$ on ice depending on the antibodies). Afterwards, the following primary antibodies were used: mouse anti-CtBP2 (1:200, BD Biosciences, Germany 612044), mouse anti-PSD-95 (1:200, Sigma Aldrich, Germany P246100ul), rabbit anti-Cav1.3 (1:100, Alomone Labs, Germany, ACC 005), rabbit anti Vglut3 (1/300, Synaptic Systems, Germany, 135 203), guinea-pig anti Vglut 3 (1/300, Synaptic Systems, Germany, 135 204), rabbit anti- otoferlin (1:100, Cedarlane/Synaptic Systems 178003(SY)), rabbit anti-KCNMA1 (BK) (1:200, Alomone Labs, Germany, APC 021). Secondary antibodies used were Alexa Fluor 488 conjugated anti-rabbit, Alexa Fluor 488 conjugated anti-guinea-pig, Alexa Fluor 568 conjugated anti-mouse, and Alexa Fluor 647 conjugated anti-rabbit (1:200, Invitrogen, Germany, A11008, A11073 A11004, A31573 respectively). For high resolution STED microscopy, STAR580 and STAR635p conjugated anti-mouse and rabbit respectively (1:200, Abberior, Germany, 2-0002-005-1 and 2-0012-007-2 respectively) have been used as secondary antibodies. Images were acquired using a Zeiss LSM800 or an Abberior Instruments Expert Line STED microscope, with excitation lasers at 488, 561, and $633 \mathrm{~nm}$ and STED lasers at $775 \mathrm{~nm}, 1.2$ W, using a 1.4 NA 100x oil immersion objective, either in confocal or in 2D-STED mode. Images were adjusted for brightness and contrast using Image $\mathrm{J}$.

\section{Data analysis}

\section{Live-imaging and IHC-patch-clamp}

The data were analyzed using custom programs in Igor Pro 6.3 (Wavemetrics, Portland, OR, USA). For analysis of IV-curves, the evoked $\mathrm{Ca}^{2+}$-currents were averaged from 5 to $10 \mathrm{~ms}$ after the start of the depolarization. $\Delta \mathrm{F}$ images were created by subtracting the fluorescence intensities inside the cell at resting state $\left(\mathrm{F}_{0}\right.$, average of 10 frames) from the ones at the depolarized state (average of 6 frames during voltage ramp protocol). $\Delta \mathrm{F}$ for each hotspot was calculated as the average of a 9 pixel square placed in the region exhibiting the greatest intensity increase within the fluorescent hotspot. Maximal $\Delta \mathrm{F}\left(\Delta \mathrm{F}_{\max }\right)$ was the average of $5 \Delta \mathrm{F}$ values obtained during the voltage ramp protocol (at the peak of $\mathrm{Ca}^{2+}$ influx). Only $\mathrm{AZs}$ presenting a $\Delta \mathrm{F}_{\max }$ greater than the average of the fluorescence intensity plus 2 standard deviations at rest were considered for further analysis. For analysis of the voltage dependence of synaptic $\mathrm{Ca}^{2+}$ signals, raw traces were fitted 
to the following function

$$
F(V)=F_{0}+\frac{f_{v} \cdot\left(V_{r}-V\right)}{1+e^{\frac{\left(V_{h}-V\right)}{k}}}
$$

where $f_{v}$ is the fluorescence-voltage-relationship $\Delta \mathrm{F} / \Delta \mathrm{V}$ obtained by linear fitting to the $\mathrm{FV}$-curve in the range of 3 to $23 \mathrm{mV}, V_{r}$ the reversal potential of the IV (after LJ potential correction), and $V$ the command voltage, in order to obtain $V_{h}$, the voltage value of half-maximal activation, and $k$, the slope factor or voltage sensitivity. The FV-fitting traces were subsequently divided by a line, which approximated the decline of fluorescence at depolarized voltages which results from the declining driving force despite full activation of the calcium channels. The line was then extended to all the voltages of the corresponding FV trace to estimate the assumed fluorescence intensity of every voltage in the full activation condition, giving the fractional activation curves. The fractional activation curves were then fitted by the Boltzmann function to obtain the parameters: voltages for half activation $\left(\mathrm{V}_{\mathrm{h}}\right)$ and slope-factor $(\mathrm{k})$. Synaptic ribbon fluorescence (visualized with the TAMRA-conjugated CtBP2/RIBEYE-binding dimer peptide) was estimated by measuring the ratio of the strongest fluorescence pixel intensity to that of the pixel nearby (8-9 pixels away inside the cell) $\left(\mathrm{F}_{\text {ribbon }} / \mathrm{F}_{\text {nearby }}\right)$. The spatial extent of the synaptic $\mathrm{Ca}^{2+}$-signals was estimated by fitting of a 2D Gaussian function to the fluorescent hotspot using a genetic fit algorithm (Sanchez del Rio and Pareschi, 2001) to obtain the full width at half maximum in the long and short axis. For each spot, the calculations were made at those confocal sections where the intensity of the spot was strongest.

In order to combine live-imaging data from multiple cells, we reconstructed the morphology of individual IHCs and the positions of their synapses based on the fluorescence of the TAMRA-conjugated RIBEYE-binding peptide and then transformed the Cartesian coordinates into cell-aligned cylindrical coordinates (for more details see(Ohn et al., 2016)). In brief, for each cell we identified the plane of symmetry orthogonal to the tonotopic axis. Then we sectioned the IHC orthogonally to a straight line fitting the pillar edge of the cell in the plane of symmetry. We calculated the center of mass for each section and connected those of the bottommost and of the largest section to define the central axis for our cylindrical coordinate system. We projected the AZ coordinates of multiple cells along their central axis for the polar charts, with the 4 sides annotated as modiolar or pillar (facing toward or away from the ganglion), and apical or basal (toward the cochlear tonotopic apex or base). 


\section{$\underline{\text { Immunostaining }}$}

Confocal and STED immunofluorescence images were analyzed and z-projected with Fiji software and further analyzed using Igor Pro. The spatial extents of the $\mathrm{Ca}^{2+-}$ channel clusters and PSD-95 was estimated in 2D STED images by assessing the full width at half maximum in the long and short axis by fitting a 2D Gaussian function (Sanchez del Rio and Pareschi, 2001). The intensities of the spots were estimated by taking the amplitude of the Gaussian fits at the confocal level. The position dependent intensity of the ribbons was analyzed by a customized algorithm in MATLAB software and used as a plug-in in Imaris. The positions of the ribbons were defined as the centers of mass of CtBP2-immunofluorescent spots. The spots were subjectively selected by thresholding the quality of a 3D Gaussian fitting, including or excluding spots. Immunofluorescence intensities were measured as the sum of the voxel values within a defined region (3 voxels in $\mathrm{X}, \mathrm{Y}$, and 2 in $\mathrm{Z}$ ) with the center of mass of the spot as origin. The cytosolic staining allowed to assign each ribbon to an IHC and to properly place the vectors. After marking the center of each IHC nucleus, a vector passing by this point and defining the central axis of the cylindrical model of the cell was adjusted to the relative orientation of each IHC cell in the XY and $\mathrm{YZ}$ axis. The Cartesian coordinates of the ribbons were transformed to cell-centric cylindrical coordinates in order to adjust differences in cellular orientation relative to the XYZ axes of the microscope. Multiple cells can be plotted by overlaying their central axes with alignment to the center of each nucleus.

\section{Statistical analysis}

The data were analyzed using Matlab (Mathworks), Igor Pro 6 (Wavemetrics), Imaris 7.6.5 (Bitplane) and Python. Averages were expressed as mean \pm standard error of the mean (S.E.M.). For every dataset, the standard deviation (S.D.), number of replicates (n) and animals $(\mathrm{N})$ were indicated. In order to compare two samples, data sets were tested for normal distribution (JarqueBera test) and equality of variances (F-test), followed by two-tailed unpaired Student's t-test, or, when data were not normally distributed and/or variance was unequal between samples, the unpaired two-tailed Mann-Whitney-Wilcoxon test was used. The non-significant difference between samples is reported as n.s., significant differences are reported as $* p<0.05$, ** $p<0.01$, $* * * p<0.001, * * * * p<0.0001$. 


\section{References}

Becker, L., Schnee, M.E., Niwa, M., Sun, W., Maxeiner, S., Talaei, S., Kachar, B., Rutherford, M.A., and Ricci, A.J. (2018). The presynaptic ribbon maintains vesicle populations at the hair cell afferent fiber synapse. ELife 7, e30241.

Beer-Hammer, S., Lee, S.C., Mauriac, S.A., Leiss, V., Groh, I.A.M., Novakovic, A., Piekorz, R.P., Bucher, K., Chen, C., Ni, K., et al. (2018). Gai Proteins are Indispensable for Hearing. Cell. Physiol. Biochem. 47, 1509-1532.

Bergstralh, D.T., Dawney, N.S., and Johnston, D.S. (2017). Spindle orientation: a question of complex positioning. Development 144, 1137-1145.

Bock, G., Gebhart, M., Scharinger, A., Jangsangthong, W., Busquet, P., Poggiani, C., Sartori, S., Mangoni, M.E., Sinnegger-Brauns, M.J., Herzig, S., et al. (2011). Functional Properties of a Newly Identified C-terminal Splice Variant of Cav1.3 L-type Ca2+ Channels. J. Biol. Chem. 286, 42736-42748.

Brandt, A., Striessnig, J., and Moser, T. (2003). CaV1. 3 channels are essential for development and presynaptic activity of cochlear inner hair cells. J. Neurosci. 23, 10832-10840.

Charizopoulou, N., Lelli, A., Schraders, M., Ray, K., Hildebrand, M.S., Ramesh, A., Srisailapathy, C.R.S., Oostrik, J., Admiraal, R.J.C., Neely, H.R., et al. (2011). Gipc3 mutations associated with audiogenic seizures and sensorineural hearing loss in mouse and human. Nat. Commun. 2, 201.

Cui, G., Meyer, A.C., Calin-Jageman, I., Neef, J., Haeseleer, F., Moser, T., and Lee, A. (2007). $\mathrm{Ca} 2+-$ binding proteins tune $\mathrm{Ca} 2+$-feedback to Cav1. 3 channels in mouse auditory hair cells. J. Physiol. 585, 791-803.

Davydova, D., Marini, C., King, C., Klueva, J., Bischof, F., Romorini, S., Montenegro-Venegas, C., Heine, M., Schneider, R., Schröder, M.S., et al. (2014). Bassoon specifically controls presynaptic P/Q-type $\mathrm{Ca}(2+)$ channels via RIM-binding protein. Neuron 82, 181-194.

Dou, H., Vazquez, A.E., Namkung, Y., Chu, H., Cardell, E.L., Nie, L., Parson, S., Shin, H.-S., and Yamoah, E.N. (2004). Null mutation of alpha1D Ca2+ channel gene results in deafness but no vestibular defect in mice. J. Assoc. Res. Otolaryngol. JARO 5, 215-226.

Ezan, J., Lasvaux, L., Gezer, A., Novakovic, A., May-Simera, H., Belotti, E., Lhoumeau, A.-C., Birnbaumer, L., Beer-Hammer, S., Borg, J.-P., et al. (2013). Primary cilium migration depends on G-protein signalling control of subapical cytoskeleton. Nat. Cell Biol. 15, 1107-1115.

Frank, T., Khimich, D., Neef, A., and Moser, T. (2009). Mechanisms contributing to synaptic $\mathrm{Ca} 2+$ signals and their heterogeneity in hair cells. Proc. Natl. Acad. Sci. 106, 4483.

Frank, T., Rutherford, M.A., Strenzke, N., Neef, A., Pangršič, T., Khimich, D., Fejtova, A., Gundelfinger, E.D., Liberman, M.C., Harke, B., et al. (2010). Bassoon and the synaptic ribbon organize $\mathrm{Ca}^{2}+$ channels and vesicles to add release sites and promote refilling. Neuron $68,724-$ 738. 
Giese, A.P., Ezan, J., Wang, L., Lasvaux, L., Lembo, F., Mazzocco, C., Richard, E., Reboul, J., Borg, J.-P., Kelley, M.W., et al. (2012). Gipc1 has a dual role in Vangl2 trafficking and hair bundle integrity in the inner ear. Development 139, 3775-3785.

Goodrich, L.V., and Strutt, D. (2011). Principles of planar polarity in animal development. Development 138, 1877-1892.

Gregory, F.D., Pangršič, T., Calin-Jageman, I.E., Moser, T., and Lee, A. (2013). Harmonin enhances voltage-dependent facilitation of $\mathrm{Ca}_{\mathrm{v}} 1.3$ channels and synchronous exocytosis in mouse inner hair cells: Harmonin regulation of $\mathrm{Ca}_{\mathrm{v}} 1.3$ and exocytosis. J. Physiol. 591, 3253-3269.

Hibino, H., Pironkova, R., Onwumere, O., Vologodskaia, M., Hudspeth, A.J., and Lesage, F. (2002). RIM - binding proteins (RBPs) couple Rab3 - interacting molecules (RIMs) to voltage gated Ca2+ channels. Neuron 34, 411-423.

Jean, P., de la Morena, D.L., Michanski, S., Tobon, L.M.J., Chakrabarti, R., Picher, M.M., Neef, J., Jung, S., Gultas, M., Maxeiner, S., et al. (2018). The synaptic ribbon is critical for sound encoding at high rates and with temporal precision. Elife 7.

Jung, S., Oshima-Takago, T., Chakrabarti, R., Wong, A.B., Jing, Z., Yamanbaeva, G., Picher, M.M., Wojcik, S.M., Göttfert, F., Predoehl, F., et al. (2015). Rab3-interacting molecules $2 \alpha$ and $2 \beta$ promote the abundance of voltage-gated $\mathrm{CaV} 1.3 \mathrm{Ca} 2+$ channels at hair cell active zones. Proc. Natl. Acad. Sci. 112, E3141-E3149.

Kantardzhieva, A., Liberman, M.C., and Sewell, W.F. (2013). Quantitative analysis of ribbons, vesicles, and cisterns at the cat inner hair cell synapse: correlations with spontaneous rate. J. Comp. Neurol. 521, 3260-3271.

Krinner, S., Butola, T., Jung, S., Wichmann, C., and Moser, T. (2017). RIM-binding protein 2 promotes a large number of $\mathrm{CaV} 1.3 \mathrm{Ca} 2+-$ channels and contributes to fast synaptic vesicle replenishment at hair cell active zones. Front. Cell. Neurosci.

Liberman, M. (1982). Single-neuron labeling in the cat auditory nerve. Science 216, 1239-1241.

Liberman, M.C. (1978). Auditory-nerve response from cats raised in a low-noise chamber. J. Acoust. Soc. Am. 63, 442-455.

Liberman, L.D., and Liberman, M.C. (2016). Postnatal maturation of auditory-nerve heterogeneity, as seen in spatial gradients of synapse morphology in the inner hair cell area. Hear. Res. 339, 12-22.

Liberman, L.D., Wang, H., and Liberman, M.C. (2011). Opposing Gradients of Ribbon Size and AMPA Receptor Expression Underlie Sensitivity Differences among Cochlear-Nerve/Hair-Cell Synapses. J. Neurosci. 31, 801-808.

Lindau, M., and Neher, E. (1988). Patch-clamp techniques for time-resolved capacitance measurements in single cells. Pflüg. Arch. Eur. J. Physiol. 411, 137-146.

Locht, C., Coutte, L., and Mielcarek, N. (2011). The ins and outs of pertussis toxin. FEBS J. 278, 4668-4682. 
Lou, X., Yano, H., Lee, F., Chao, M.V., and Farquhar, M.G. (2001). GIPC and GAIP form a complex with TrkA: a putative link between $\mathrm{G}$ protein and receptor tyrosine kinase pathways. Mol. Biol. Cell 12, 615-627.

Lu, X., and Sipe, C.W. (2016). Developmental regulation of planar cell polarity and hair-bundle morphogenesis in auditory hair cells: lessons from human and mouse genetics. Wiley Interdiscip. Rev. Dev. Biol. 5, 85-101.

Lv, C., Stewart, W.J., Akanyeti, O., Frederick, C., Zhu, J., Santos-Sacchi, J., Sheets, L., Liao, J.C., and Zenisek, D. (2016). Synaptic Ribbons Require Ribeye for Electron Density, Proper Synaptic Localization, and Recruitment of Calcium Channels. Cell Rep. 15, 2784-2795.

Mauriac, S.A., Hien, Y.E., Bird, J.E., Carvalho, S.D.-S., Peyroutou, R., Lee, S.C., Moreau, M.M., Blanc, J.-M., Gezer, A., Medina, C., et al. (2017). Defective Gpsm2/Go $\alpha_{i 3}$ signalling disrupts stereocilia development and growth cone actin dynamics in Chudley-McCullough syndrome. Nat. Commun. 8, 14907.

Maxeiner, S., Luo, F., Tan, A., Schmitz, F., and Südhof, T.C. (2016). How to make a synaptic ribbon: RIBEYE deletion abolishes ribbons in retinal synapses and disrupts neurotransmitter release. EMBO J. 35, 1098-1114.

Merchan-Perez, A., and Liberman, M.C. (1996). Ultrastructural differences among afferent synapses on cochlear hair cells: correlations with spontaneous discharge rate. J. Comp. Neurol. $371,208-221$.

Meyer, A.C., Frank, T., Khimich, D., Hoch, G., Riedel, D., Chapochnikov, N.M., Yarin, Y.M., Harke, B., Hell, S.W., Egner, A., et al. (2009). Tuning of synapse number, structure and function in the cochlea. Nat. Neurosci. 12, 444-453.

Moser, T., and Beutner, D. (2000). Kinetics of exocytosis and endocytosis at the cochlear inner hair cell afferent synapse of the mouse. Proc. Natl. Acad. Sci. U. S. A. 97, 883-888.

Neef, J., Urban, N.T., Ohn, T.L., Frank, T., Jean, P., Hell, S.W., Willig, K.I., and Moser, T. (2018). Quantitative optical nanophysiology of $\mathrm{Ca} 2+$ signaling at inner hair cell active zones. Nat. Commun. 9.

Ohlemiller, K.K., Echteler, S.M., and Siegel, J.H. (1991). Factors that influence rate-versusintensity relations in single cochlear nerve fibers of the gerbil. J. Acoust. Soc. Am. 90, 274-287.

Ohn, T.-L., Rutherford, M.A., Jing, Z., Jung, S., Duque-Afonso, C.J., Hoch, G., Picher, M.M., Scharinger, A., Strenzke, N., and Moser, T. (2016). Hair cells use active zones with different voltage dependence of $\mathrm{Ca} 2+$ influx to decompose sounds into complementary neural codes. Proc. Natl. Acad. Sci. 113, 201605737.

Petitpré, C., Wu, H., Sharma, A., Tokarska, A., Fontanet, P., Wang, Y., Helmbacher, F., Yackle, K., Silberberg, G., Hadjab, S., et al. (2018). Neuronal heterogeneity and stereotyped connectivity in the auditory afferent system. Nat. Commun. 9, 3691.

Picher, M.M., Oprişoreanu, A.-M., Jung, S., Michel, K., Schoch, S., and Moser, T. (2017). Rab Interacting Molecules 2 and 3 Directly Interact with the Pore-Forming CaV1.3 Ca2+ Channel Subunit and Promote Its Membrane Expression. Front. Cell. Neurosci. 11. 
Platzer, J., Engel, J., Schrott-Fischer, A., Stephan, K., Bova, S., Chen, H., Zheng, H., and Striessnig, J. (2000). Congenital deafness and sinoatrial node dysfunction in mice lacking class D L-type Ca2+ channels. Cell 102, 89-97.

Probst, F.J., Fridell, R.A., Raphael, Y., Saunders, T.L., Wang, A., Liang, Y., Morell, R.J., Touchman, J.W., Lyons, R.H., Noben-Trauth, K., et al. (1998). Correction of deafness in shaker2 mice by an unconventional myosin in a BAC transgene. Science 280, 1444-1447.

Pyott, S.J. (2004). Extrasynaptic Localization of Inactivating Calcium-Activated Potassium Channels in Mouse Inner Hair Cells. J. Neurosci. 24, 9469-9474.

Ruel, J., Nouvian, R., D’Aldin, C.G., Pujol, R., Eybalin, M., and Puel, J.-L. (2001). Dopamine inhibition of auditory nerve activity in the adult mammalian cochlea. Eur. J. Neurosci. 14, 977986.

Russell, I., and Sellick, P. (1983). Low-frequency characteristics of intracellularly recorded receptor potentials in guinea-pig cochlear hair cells. J. Physiol. 338, 179-206.

Sanchez del Rio, M., and Pareschi, G. (2001). Global optimization and reflectivity data fitting for X-ray multilayer mirrors by means of genetic algorithms. SPIE Proc. 88-96.

Sendin, G., Bulankina, A.V., Riedel, D., and Moser, T. (2007). Maturation of ribbon synapses in hair cells is driven by thyroid hormone. J. Neurosci. Off. J. Soc. Neurosci. 27, 3163-3173.

Sheets, L., Trapani, J.G., Mo, W., Obholzer, N., and Nicolson, T. (2011). Ribeye is required for presynaptic $\mathrm{CaV1.3a}$ channel localization and afferent innervation of sensory hair cells. Development 138, 1309-1319.

Sheets, L., He, X.J., Olt, J., Schreck, M., Petralia, R.S., Wang, Y.-X., Zhang, Q., Beirl, A., Nicolson, T., Marcotti, W., et al. (2017). Enlargement of Ribbons in Zebrafish Hair Cells Increases Calcium Currents But Disrupts Afferent Spontaneous Activity and Timing of Stimulus Onset. J. Neurosci. 37, 6299-6313.

Shrestha, B.R., Chia, C., Wu, L., Kujawa, S.G., Liberman, M.C., and Goodrich, L.V. (2018). Sensory Neuron Diversity in the Inner Ear Is Shaped by Activity. Cell 174, 1229-1246.e17.

Sun, S., Babola, T., Pregernig, G., So, K.S., Nguyen, M., Su, S.-S.M., Palermo, A.T., Bergles, D.E., Burns, J.C., and Müller, U. (2018). Hair Cell Mechanotransduction Regulates Spontaneous Activity and Spiral Ganglion Subtype Specification in the Auditory System. Cell 174, 12471263.e15.

Tan, B.Z., Jiang, F., Tan, M.Y., Yu, D., Huang, H., Shen, Y., and Soong, T.W. (2011). Functional characterization of alternative splicing in the C-terminus of L-type CaV1.3 channels. J. Biol. Chem.

Tarchini, B., Jolicoeur, C., and Cayouette, M. (2013). A Molecular Blueprint at the Apical Surface Establishes Planar Asymmetry in Cochlear Hair Cells. Dev. Cell 27, 88-102.

Tarchini, B., Tadenev, A.L.D., Devanney, N., and Cayouette, M. (2016). A link between planar polarity and staircase-like bundle architecture in hair cells. Development 143, 3926-3932. 
Winter, I.M., Robertson, D., and Yates, G.K. (1990). Diversity of characteristic frequency rateintensity functions in guinea pig auditory nerve fibres. Hear. Res. 45, 191-202.

Wong, A.B., Rutherford, M.A., Gabrielaitis, M., Pangršič, T., Göttfert, F., Frank, T., Michanski, S., Hell, S., Wolf, F., Wichmann, C., et al. (2014). Developmental refinement of hair cell synapses tightens the coupling of Ca2+ influx to exocytosis. EMBO J. 33, 247-264.

Yin, Y., Liberman, L.D., Maison, S.F., and Liberman, M.C. (2014). Olivocochlear innervation maintains the normal modiolar-pillar and habenular-cuticular gradients in cochlear synaptic morphology. J. Assoc. Res. Otolaryngol. JARO 15, 571-583.

Zhang, L., Engler, S., Koepcke, L., Steenken, F., and Köppl, C. (2018). Concurrent gradients of ribbon volume and AMPA-receptor patch volume in cochlear afferent synapses on gerbil inner hair cells. Hear. Res. 364, 81-89. 

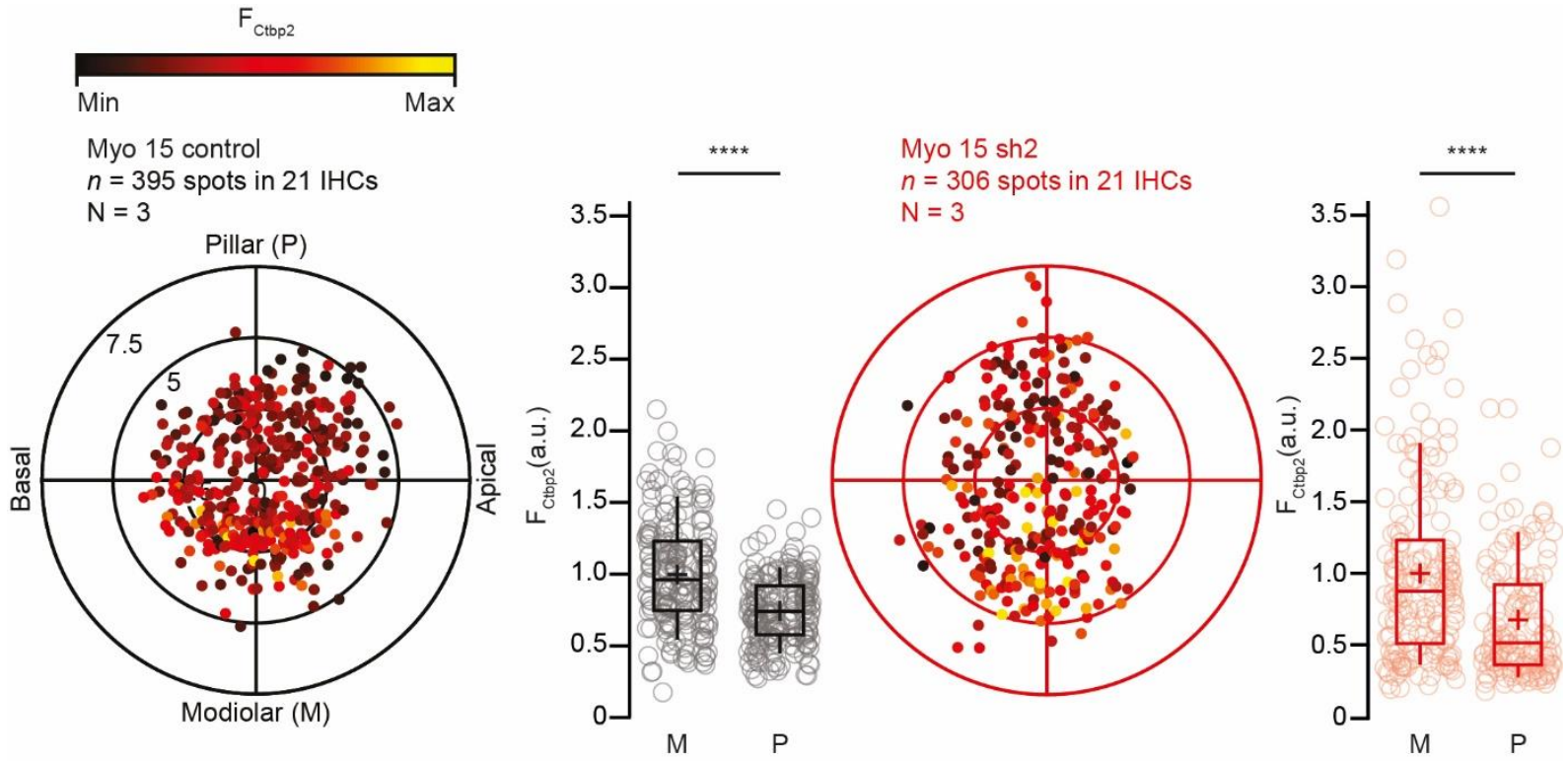

Figure supplement 1: Myo15 sh2 mice preserve their modiolar-pillar gradient for ribbon size

The polar charts display locations and intensities of immunofluorescently labelled CtBP2 marking the ribbons. Modiolar and pillar refer to facing to or from the ganglion in the modiolus, apical and basal refer to the tonotopic axis of the organ of Corti. The fluorescence intensity of each CtBP2 signal point is reflected by its color, with warmer, yellow tones indicating higher intensity, and cooler, darker tones indicating lower intensity. Each radial circle is $2.5 \mu \mathrm{m}$. The IHCs from both Myo 15 control and Myo15 sh2 mutant conditions display a significant gradient of stronger ribbons in the modiolus (M) as compared to the pillar side (P) (Myo 15 control (black): modiolar: $n=203$ AZs vs Pillar: $n=193$ AZs, Myo 15 sh 2 mutant (red): modiolar: $n=173$ AZs vs Pillar: $n=133$ AZs, $p<0.00001$ Mann-Whitney-Wilcoxon test for both conditions). 
A

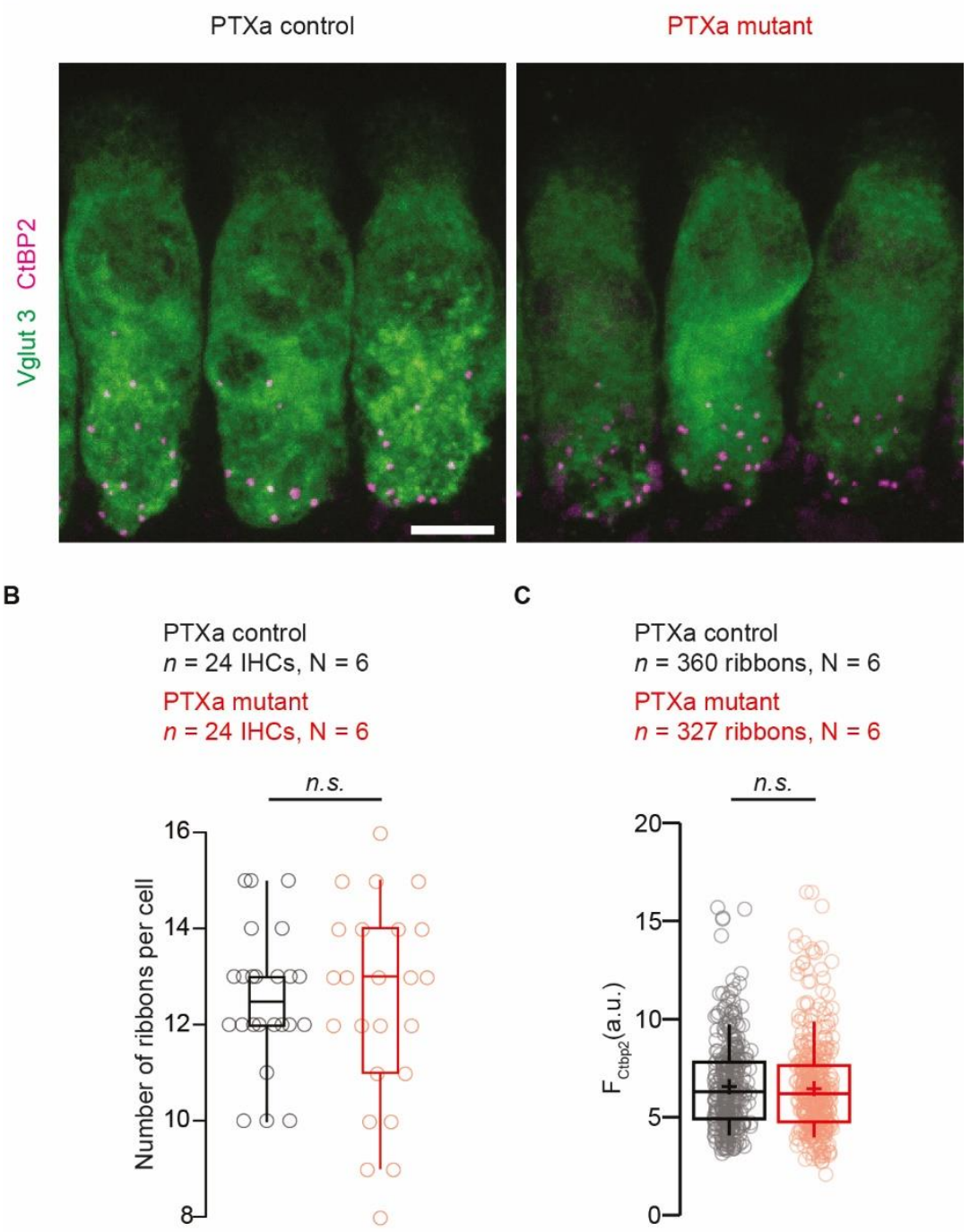

Figure supplement 2: Disruption of Gai signaling does not change ribbon size and number (A) Maximal projection of confocal sections from organs of Corti co-immunolabeled for the IHC marker Vglut3 (green) and the ribbon marker CtBP2 (magenta). Scale bar $=5 \mu \mathrm{m}$. (B) The average number of ribbons per cell is comparable between PTXa control and mutant conditions ( $n=24, \mathrm{~N}=6$ for both conditions, $p=0.81$, t.test). Box plots show 10, 25, 50, 75 and 90th percentiles with individual data points overlaid, means are shown as crosses as for (C). (C) The average intensity of the ribbons is comparable between PTXa control and mutant conditions (PTXa control: $n=327$, PTXa mutant: $n=360 ; \mathrm{N}=9$ in both conditions, $p=0.28$, Mann-Whitney-Wilcoxon test). 
A

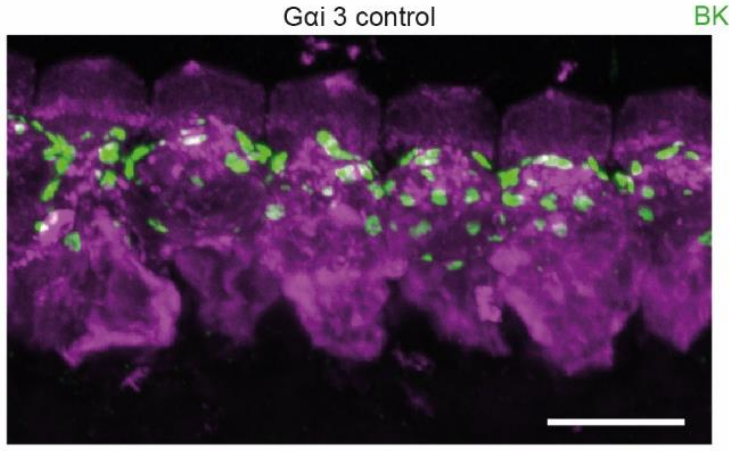

BK Myo7

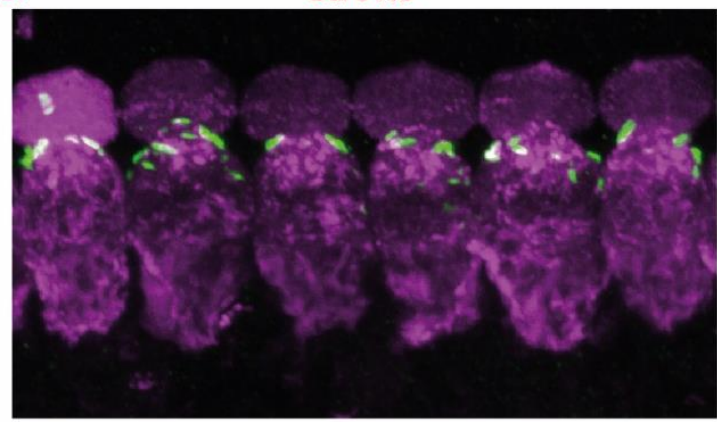

B

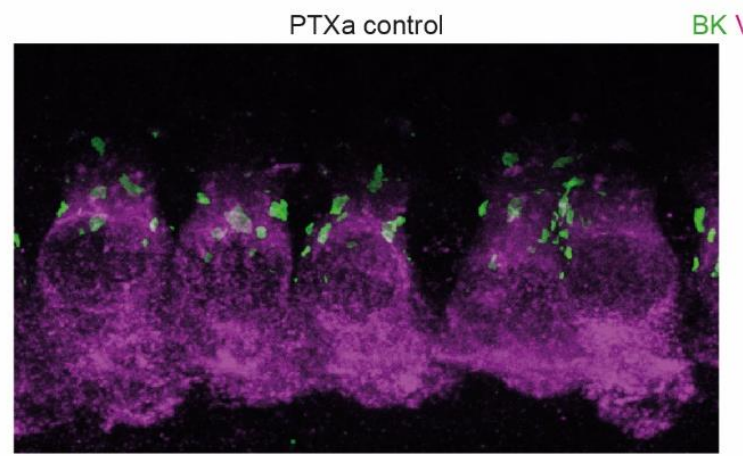

BK Vglut 3

PTXa mutant

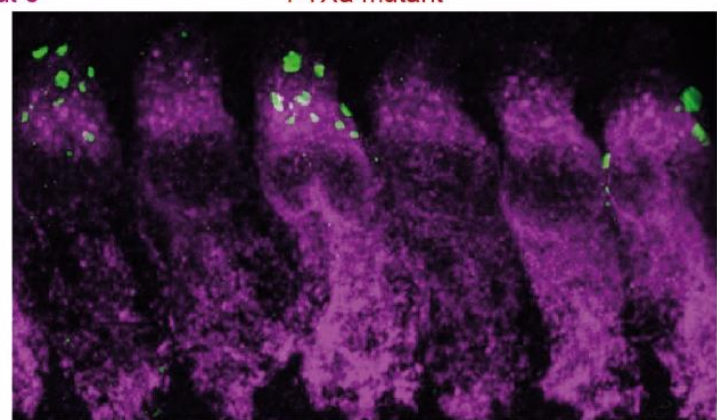

Figure supplement 3: Immunostaining study of the BK channel clustering at Gai3 and PTXa mutant inner hair cells

(A) Maximal projection of confocal sections from organs of Corti co-immunolabeled for the largeconductance $\mathrm{Ca}^{2+}$ activated $\mathrm{K}^{+}(\mathrm{BK})$ channels (green as for $\mathrm{B}, \mathrm{C}$ ) and the $\mathrm{HC}$ marker Myo7 (magenta as for B). The BK positive spots are still present in the Gai $3 \mathrm{KO}$ IHCs, but in smaller amount. Scale bar $=5$ $\mu \mathrm{m}$ as for (B). (B) The BK channels are co-stained with the IHC marker Vglut3, exhibiting a mosaic expression in the PTXa mutant IHCs having normal clustering or no signal. 


\section{Discussion}

In this thesis, I provide further insight into the structure and function of the afferent IHC synapse that mediates the encoding of sound in the cochlea. First, my collaborators and I investigated the role of the hallmark feature of the IHC AZ, the synaptic ribbon. The constitutive and specific deletion of the gene coding for the protein RIBEYE (Maxeiner et al., 2016), the major and only known specific component of the ribbon (Schmitz et al., 2000), resulted in the complete disappearance of the synaptic ribbons in the cochlea as indicated by immunofluorescence and electron microscopy (Becker et al., 2018; Jean et al., 2018). This manipulation resulted in a compensatory mechanism reorganizing each IHC AZ into several small ribbonless AZs juxtaposed to a single enlarged PSD. Despite this compensation, we showed a reduced temporal precision of sound coding and impaired vesicle replenishment of the ribbonless AZ at high rates of stimulation, indicating a conveyor belt function of the synaptic ribbon (Jean et al., 2018).

Then, my work focused on characterizing the cellular mechanisms responsible for the position-dependent differences of $\mathrm{AZs}$ in ribbon size and $\mathrm{Ca}^{2+}$ influx properties, thought to drive the diverse firing behaviors of type I SGNs. First, together with the team of Matthew Kelley, we showed that the transcription factor Pou4f1 is expressed by 30\% of type I SGNs after hearing onset, and that most of them ( $95 \%)$ form a synaptic contact with the modiolar side of the IHC. We proposed that these SGNs represent low SR, high threshold SGNs instructing presynaptic properties, as disruption of postsynaptic Pou4f1 led to more hyperpolarized activation of presynaptic $\mathrm{Ca}^{2+}$ channels by increasing their voltage-sensitivity. Moreover, the modiolar-pillar gradient of $\mathrm{AZ} \mathrm{Ca}^{2+}$ influx strength collapsed, especially when the winning $\mathrm{AZs}$ (AZs with the strongest $\mathrm{Ca}^{2+}$ influx per cell) were not taken into account in the analysis. This suggests a role for postsynaptic Pou4F1 in instructing a higher number of $\mathrm{Ca}^{2+}$ channels and, potentially, more depolarized activation range at modiolar IHC AZs other than the winning AZ.

Next, in collaboration with the laboratory of Basile Tarchini, we explored whether the planar polarity mechanisms establishing hair bundle orientation and cell apical surface asymmetry across tissue could influence the basolateral gradient of IHC AZ properties. We indicated a role for the Gai/LGN complex in setting this modiolar-pillar gradient of $\mathrm{Ca}^{2+}$ channel number per $\mathrm{AZ}$, independent of the developmental kinocilium shift that supposedly dictates hair bundle orientation. Blocking Gai signaling by HC specific expression of the pertussis toxin (PTXa) (Tarchini et al., 2016) increased the sizes of $\mathrm{Ca}^{2+}$ channel clusters and PSDs. Functionally, we 
found an augmented maximal amplitude as well as an hyperpolarized activation of the whole-cell and the synaptic $\mathrm{Ca}^{2+}$ influx. Moreover, we observed a collapse of the modiolar/pillar gradient for maximal synaptic $\mathrm{Ca}^{2+}$ influx. However, in both Pou4F1 and PTXa mutant animals, the pillarmodiolar gradient of the voltage-dependent activation of the synaptic $\mathrm{Ca}^{2+}$ influx was preserved, indicating distinct mechanisms governing strength and voltage-dependent activation of the synaptic $\mathrm{Ca}^{2+}$ influx. Here, I will consider the potential presynaptic, postsynaptic and efferent mechanisms responsible for setting the diverse firing characteristics of the SGNs and, thereby, permitting a wide dynamic range of sound encoding. I will also discuss the requirements for future studies in order to improve the currently still incomplete understanding of these mechanisms.

\subsection{Cellular mechanisms of wide dynamic range of sound encoding}

\subsubsection{Presynaptic candidate mechanisms}

In mammals each presynaptic $\mathrm{AZ}$ is thought to deliver the exclusive sensory input to its postsynaptic afferent SGN (Nadol, 1988). The first evidence for a presynaptic mechanism came from observations in cats that SGNs of different firing characteristics receive input from presynaptic AZs with distinct morphologies (Merchan-Perez and Liberman, 1996). By in vivo extracellular recordings of spontaneous and sound evoked SGN firing rates, followed by back tracing labelling and EM reconstruction of the spatial IHC connectivity of the afferents, this study showed that low-SR fibers connected to modiolar AZs that tend to have larger synaptic ribbons with more synaptic vesicles as compared to pillar AZs. A later EM study, still in the cat, revealed that modiolar ribbons (thought to be presynaptic to low SR SGNs) tend to be longer and thinner and tether more synaptic vesicles than pillar ribbons (associated with high SR SGNs, (Kantardzhieva et al., 2013). However, the restricted number of replicates in these studies leaves uncertainty concerning how generalizable these observations are. Moreover, we do not know to which degree they apply to other animal models.

Recent studies in the mouse cochlea demonstrated that synaptic $\mathrm{Ca}^{2+}$ signals at IHC synapses were highly heterogeneous in terms of amplitude and voltage-dependence of activation (Frank et al., 2009). Moreover by immunofluorescence microscopy and patch-clamp combined with $\mathrm{Ca}^{2+}$ imaging it was shown that the modiolar AZs present bigger ribbons and $\mathrm{Ca}^{2+}$ channel clusters, associated with a stronger maximal $\mathrm{Ca}^{2+}$ influx with, however, more depolarized 
activation when compared to the pillar side AZs (Meyer et al., 2009; Ohn et al., 2016). The heterogeneity of presynaptic $\mathrm{Ca}^{2+}$ signals at IHCs is a seductive candidate mechanism for the firing diversity of the SGNs. Contrary to a neuron, the IHC is considered to be isopotential without apparent compartmentalization. How an IHC manages to differentially distribute $\mathrm{Ca}^{2+}$ channels and other synaptic components to individual AZs is a fascinating and still enigmatic question. The number of $\mathrm{Ca}^{2+}$ channels and their functional properties at each presynaptic $\mathrm{AZ}$ are governed by the expression of specific subunits and splice variants as well as by that of scaffold/interacting proteins tethering $\mathrm{Ca}^{2+}$ channels to the $\mathrm{AZ}$ and/or regulate their function and abundance by direct or indirect interaction. For instance, the auxiliary subunits Cavß2 and $\mathrm{Cav}_{2} \delta_{2}$ were previously shown to be required for normal $\mathrm{Ca}^{2+}$ current amplitude and exocytosis at IHCs (Fell et al., 2016; Neef et al., 2009). Disrupting the function of the distal C-terminal regulatory domain of Cav1.3 did enhance maximal synaptic $\mathrm{Ca}^{2+}$ influx but did not noticeably alter the heterogeneity of voltage-dependent activation of $\mathrm{Ca}^{2+}$ influx or of sound encoding properties of SGNs (Ohn et al., 2016). Work in zebrafish HCs has revealed an interesting molecular interaction between RIBEYE and Cav1.3 channels. Indeed, Ribeye knock-down led to a loss of presynaptic Cav1.3a $\mathrm{Ca}^{2+}$ channel clusters in HCs. By contrast, overexpression of RIBEYE resulted in Cav1.3a channels colocalizing with ectopic aggregates of RIBEYE protein (Sheets et al., 2011). Moreover, HCs with enlarged ribbons exhibited increased global and ribbon localized $\mathrm{Ca}^{2+}$ influx (Sheets et al., 2017). Finally, still in the zebrafish, a recent study introduced frameshift mutations to remove RIBEYE and this induced a mislocalization of $\mathrm{Ca}^{2+}$ channels despite the presence of "ghost ribbons" that were poorly localized to synapses and $\mathrm{Ca}^{2+}$ channels (Lv et al., 2016).

In the mouse retina, loss of ribbons loosened the coupling of $\mathrm{Ca}^{2+}$ channels with the release sites (Maxeiner et al., 2016), while in the cochlear IHCs no such coupling defect was evident (Becker et al., 2018). In this thesis, our work put in evidence a compensatory mechanism leading to the transformation of one ribbon AZ to multiple small ribbonless AZs (Jean et al., 2018, see Figure 8). Such synapse reshaping in IHCs of the constitutive Ribeye KO might explain the modest functional phenotype that included a lack of coupling defect between $\mathrm{Ca}^{2+}$ channels and release sites. We did, however, observe a depolarized shift in the voltage-dependence of $\mathrm{Ca}^{2+}$ channel activation, increasing the proportion of low SR fibers and elevating their threshold. To avoid compensatory mechanisms, future studies should remove the ribbons by cre-inducible disruption of Ribeye after the synaptic ultrastructure becomes mature. Then, a role for the 
synaptic ribbon in setting up the modiolar-pillar arrangement of synaptic $\mathrm{Ca}^{2+}$ influx properties could be investigated.

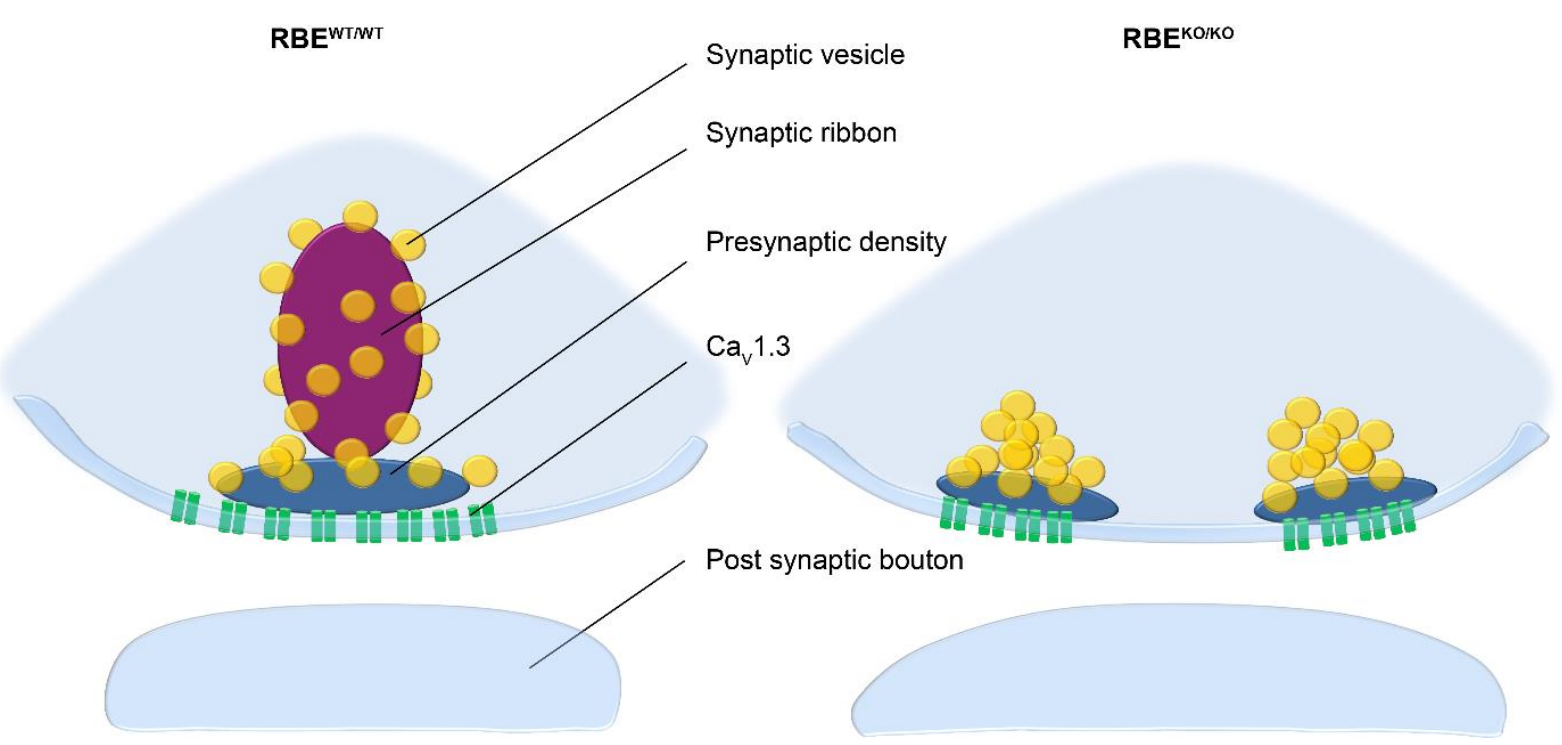

\section{Figure 8: $\mathrm{AZ}$ reorganization in the constitutive Ribeye $\mathrm{KO}$}

Schematic illustration of the IHC synapse in control condition and with specific deletion of RIBEYE protein. After constitutive deletion of the synaptic ribbon, the conventional IHC AZ is reorganized in multiple ribbonless AZs made of a pile of vesicles anchored to a PD, juxtaposed to one enlarged PSD. Not to scale.

Bassoon, RIM and RIM-BP positively regulate the number of $\mathrm{Ca}^{2+}$ channels at the AZ (Frank et al., 2010; Jung et al., 2015; Krinner et al., 2017). Indeed RIM2 $\alpha$ and $\beta$ have been reported to promote the clustering of these channels at the synapse (Jung et al., 2015), and RIM2 and RIM3 were shown to directly interact with the pore-forming subunit of $\mathrm{Cav} 1.3 \mathrm{Ca}^{2+}$ channel (Picher et al., 2017a). This regulation can also be indirect via RBPs (Davydova et al., 2014) which were previously shown to interact with $\mathrm{Cav} 1.3 \mathrm{Ca}^{2+}$ channels (Hibino et al., 2002) and are required to set a normal $\mathrm{Ca}^{2+}$ channel complement at the IHC AZ (Krinner et al., 2017). Another candidate is Harmonin that regulates these channels likely by binding one of its PDZ domain to the ITTL motif of the extreme Cav1.3 C-terminus. Harmonin mutant IHCs exhibited weaker ribbons as well as reduced whole-cell and synaptic $\mathrm{Ca}^{2+}$ influx, which activated at more negative potentials. However, this hyperpolarized shift of $\mathrm{Ca}^{2+}$ channel activation was observed for both modiolar and pillar sides, arguing against a contribution to the spatially organized presynaptic heterogeneity (Ohn et al., 2016). The disruption of another PDZ-protein, Gipc3, reversed the normal modiolar-pillar gradient of maximal $\mathrm{AZ} \mathrm{Ca}^{2+}$ influx, whereas the one for voltagedependent activation was maintained. $\mathrm{Ca}^{2+}$ channel activation was shifted to more hyperpolarized 
potentials and, in parallel, the fraction of SGNs with high-spontaneous firing rate and enhanced sound-onset firing was, potentially as a consequence, increased. How Gipc3 protein might be involved directly or indirectly for establishing this gradient remains to be elucidated. In view of a possible analogy to Gipc1, Gipc3 could adapt motor proteins to the $\mathrm{Ca}^{2+}$ channels, trafficking them preferentially to modiolar AZs. The trafficking mechanisms of the synaptic components to the AZs are still enigmatic. Planar polarity pathways governing hair bundle orientations and apical surface asymmetry in the organ of Corti are promising mechanisms to contribute to the common modiolar location of the strongest synapses, and should be investigated in further detail. Kif3a, shown to be a component of the retinal synaptic ribbon (Muresan et al., 1999) and essential for kinocilium formation in cochlear HCs (Sipe and Lu, 2011), as well as Clarin-1, a tetraspanlike membrane protein essential for hair bundle morphogenesis and AZ organization (Dulon et al., 2018) seem to be promising candidates for establishing presynaptic heterogeneity. In this thesis, we demonstrated a role for Gai proteins and their direct interacting partner LGN, where the disruption of their signaling resulted in $\mathrm{AZ}$ reorganization and loss of spatial gradient for ribbon and synaptic $\mathrm{Ca}^{2+}$ influx strength (Figure 9). More work on Gai proteins remains to be done, focusing on single and multiple KOs for different Gai subunits, interacting partners and receptors, with deeper investigations combining EM study of the synapse ultrastructure, live $\mathrm{Ca}^{2+}$ -imaging experiments at IHC AZs and in vivo single unit recordings of SGNs.

\subsubsection{Postsynaptic candidate mechanisms}

The postsynaptic SGN I boutons could differ in the number and/or properties of their glutamate receptors and their voltage-gated ion channels leading to differences in the postsynaptic response and electrical excitability. For example, a given amount of neurotransmitter release might depolarize the high-AMPAR-content SGNs above threshold, whereas the same amount of glutamate would fail in the low-AMPAR-content SGNs. However, the expected outcome would be a scaling of SR with evoked spike-rates. This, however, does not agree with the observation that SGNs with different SRs have comparable adapted peak rates over a very broad range of SRs (Liberman, 1978). Interestingly, a negative correlation between the sizes of ribbons and AMPA receptor clusters have been found in mice (Liberman et al., 2011) with smaller AMPAR clusters present on the modiolar IHC side (Yin et al., 2014), while the opposite observation was made in the guinea pig (Zhang et al., 2018). 


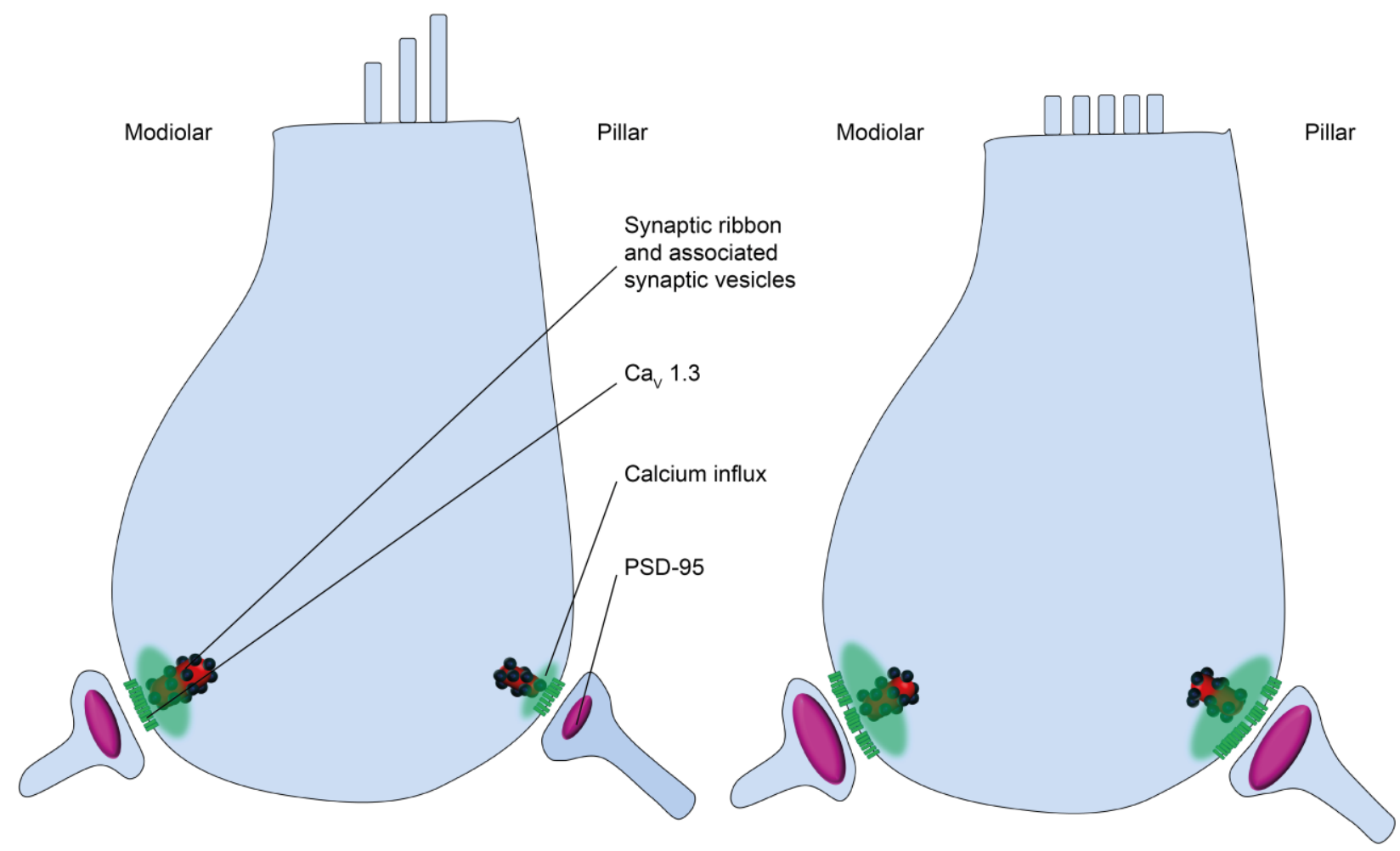

Figure 9: Blockade of Gai signaling enlarges the synapses and collapses the spatial gradient for $\mathrm{AZ}$ strength

Schematic representation of the IHCs and afferent synapses. The HC specific expression of PTXa, blocking Gai signaling, potentiates the synaptic strength resulting in an increased and hyperpolarized $\mathrm{Ca}^{2+}$ influx at the whole-cell and single synapse levels. The modiolar/pillar gradient for AZ strength present in control condition collapses in the presence of PTX; however, the spatial gradient for the voltage activation of the $\mathrm{Ca}^{2+}$ influx remains. Not to scale.

The excitability of SGNs is another possible mechanism leading to heterogeneous response properties. High-SR fibers exhibit thick (0.8-1.2 $\mu \mathrm{m}$ diameter) and mitochondrion-rich peripheral processes, while low-SR SGNs have thinner (0.3-0.8 $\mu \mathrm{m}$ diameter) and mitochondrion-poor processes (Liberman, 1982). The differences in surface to volume ratio could influence the length constant of electrotonic spread along the fiber, and thus affect excitability. However, it is unclear whether a larger fiber diameter associated with lower internal resistance is responsible for these different firing characteristics. Moreover, there are indications for heterogeneity among the SGNs regarding the content of various voltage-gated ion channel controlling resting membrane potential, action potential duration, threshold of activation and latency to the first spike as well as accommodation, varying along the tonotopic axis (Adamson 
et al., 2002; Bakondi et al., 2008; Chen et al., 2011; Liu et al., 2014; Mo and Davis, 1997).

Performing single-cell transcriptomics, two parallel studies (Shrestha et al., 2018; Sun et al., 2018) characterized three type I SGN subtypes in mice, expressing distinct cohorts of ion channels, receptors, synaptic proteins, and adhesion molecules that vary along the tonotopic axis. Interestingly, those three subtypes differentially connect to the IHCs along the modiolar-pillar axis in mice. By assuming analogy, these molecularly defined subtypes were related to functionally defined categories of type I SGN that also exhibit such differential IHC innervation as shown by in vivo single unit recordings of the SGNs in the cat (Liberman, 1982; MerchanPerez and Liberman, 1996). The neurons innervating the pillar side of the IHC express high levels of neurofilament and mitochondrial genes, indicating elevated mitochondrial content and axon diameter as reported in cats. These distinct identities emerged during the first postnatal week in an activity-dependent manner. Interestingly, disrupting glutamate release by inactivating Vglut3 caused the predominant loss of the modiolar targeting SGN subtype, while suppressing mechanotransduction increased the fraction of SGNs co-expressing subtype markers, as found at immature stages. A third study (Petitpré et al., 2018) used a similar approach and also characterized 3 subtypes of type I SGNs expressing different transcripts and differentially targeting the IHC base. Performing whole-cell patch clamp recordings from dissociated SGNs, they showed that pillar side associated SGNs (based on marker) exclusively exhibit a unitary spike accommodation (trigger a single action potential during stimulus: phasic firing), while the modiolar side associated SGNs were additionally displaying multiple spike accommodation (trigger several action potentials during stimulus: tonic firing), difference potentially explained by the differential expression of Kcnc2 (Kv3.2) in this subpopulation. In this thesis, our work investigated more deeply in one of this modiolar subpopulation marker at different stages, the transcription factor Pou4f1. We propose that Pou4f1 defines the low SR, high threshold fate of SGNs by instructing an increased voltage sensitivity and a greater complement of the $\mathrm{Ca}^{2+}$ channels in the associated presynaptic AZ. Other specific markers for modiolar and pillar targeting SGNs have been characterized (Petitpré et al., 2018; Shrestha et al., 2018; Sun et al., 2018), and additional works characterizing the presynaptic ultrastructure, gradient of synapse properties as well as postsynaptic molecular identities and SGN firing properties in their absence will be essential. 


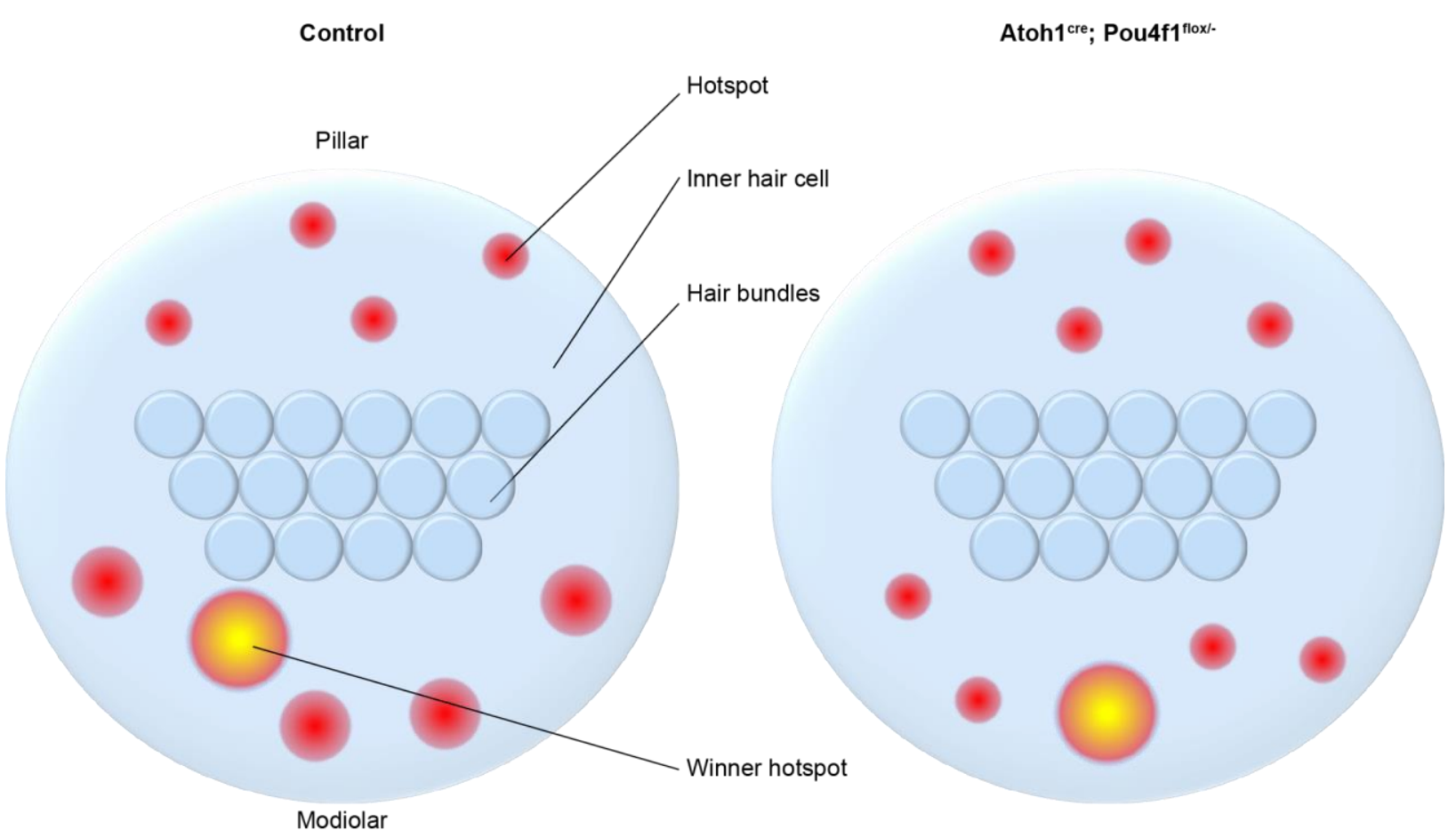

Figure 10: Pou4f1 is not required for the formation of the winning $\mathrm{AZ}$, but contributes to setting the number of $\mathrm{Ca}^{2+}$ channels at the other modiolar $\mathrm{AZs}$

Schematic top view of the IHCs. The $\mathrm{Ca}^{2+}$ hotspots from one IHC are depicted in control and mutant conditions, with the size reflecting their associated $\mathrm{Ca}^{2+}$ influx amplitude. Pou4f1 deletion (Atoh1 $1^{\text {cre; }}$; Pou $4 \mathrm{f}^{\text {flox/-) }}$ ) from the SGNs does not impact the winning AZ (yellow), however the other modiolar AZs are losing their stronger $\mathrm{Ca}^{2+}$ influx as compared to the pillar side AZs. Not to scale.

\subsubsection{Efferent candidate mechanisms}

Originating from the brainstem, medial olivocochlear (MOC) and lateral olivocochlear (LOC) efferent fibers, at adult stages, make synaptic contacts with OHCs and type I SGNs respectively. Right after birth, LOC efferent neurons form inhibitory synaptic contacts with IHCs (Glowatzki and Fuchs, 2000), and then disappear at the onset of hearing around P12 in rodents (Katz et al., 2004). At that stage, LOC efferent fibers are still connected to type I SGNs below the IHCs, upstream of the first heminode, where spike generation is thought to happen. However, the precise role of efferent modulation of SGNs, and putatively of IHCs in mature mice is still enigmatic. It has been shown that both LOC and MOC efferents are positive for cholinergic markers (Maison et al., 2003; Sobkowicz and Emmerling, 1989). For example, all LOC efferents show choline acetyltransferase immunolabelling (Felix and Ehrenberger, 1992). Moreover, dopaminergic receptors have been reported in the cochlear SGNs (Maison et al., 2012), and some LOC efferents appear to express the enzyme tyrosine hydroxylase enabling dopamine synthesis 
(Eybalin et al., 1993). Interestingly, in vivo studies in the guinea pig assessing the LOC modulation onto SGN function led to diverging conclusions. A first study applying acetylcholine and gamma-aminobutyric acid in the cochlea reported an increase and decrease in SR, respectively, proposing an excitatory role of the LOC pathway (Felix and Ehrenberger, 1992). A second study, perfusing dopamine and selective antagonists proposed a tonic inhibitory action (Ruel et al., 2001). Finally, a last study stimulating the LOC pathway and performing selective lesions of central somata or axon tracts, showed both an increase and decrease of the afferent neural responses (Groff and Liberman, 2003). These discrepancies suggest both a stimulating and inhibiting role of the LOC efferent system onto the auditory nerve, by yet to be defined synaptic mechanisms. These observations suggest a complex neurochemistry at those synapses, supported by the co-existence of different neurotransmitter systems in the LOC pathway and their further diversification by peptidergic co-transmitters (Eybalin, 1993; Safieddine and Eybalin, 1992, for review see Ruel et al., 2007).

Previous studies in cats (Liberman, 1980; Liberman et al., 1990) and mice (Ohn et al., 2016; Yin et al., 2014) reported approximately twice the number of synapses on the modiolar side than on the pillar side of the IHCs. Since high-SR fibers outnumber low-SR by 60 versus $40 \%$ (Liberman, 1978), the spatial segregation of SR groups cannot be exclusively based on afferent synapse location onto the IHCs. Moreover, efferent synapses were found to be particularly numerous on SGNs with thin peripheral processes in cats, which are supposed to present the morphological correlate of low-SR/high-threshold SGNs (Liberman, 1980; Liberman et al., 1990; Merchan-Perez and Liberman, 1996). Consequently, it could be hypothesized that tonic inhibition of SGNs contributes to establishing low SR and high thresholds of SGNs. However, lesioning LOC did not alter the fundamental relation between SR and threshold (Liberman, 1990), arguing against tonic inhibition being the sole mechanism for SR differences. Interestingly, the modiolar-pillar gradient of ribbon and AMPAR patch sizes was lost upon lesion of the LOC efferents (Yin et al., 2014). Evaluating the spatial heterogeneity of synaptic $\mathrm{Ca}^{2+}$ influx properties at the IHC without efferent modulation would be of great interest. This would require surgical or genetic disruption of the LOC system (Yin et al., 2014) in combination with subcellular $\mathrm{Ca}^{2+}$ imaging as reported in this thesis. Whether the LOC efferents may have a direct or indirect (e.g. retrograde via the SGNs) effect on the IHCs remains to be investigated in future studies. 


\subsection{Outlook}

The cellular mechanisms underlying the sound encoding over the broad dynamic range of audible sound pressures remain only partially understood. The different findings from the literature agree on some points but also disagree on many. Often they are hard to reconcile due to different techniques, analysis and animal models. A considerable amount of work remains to be done to establish a clear and precise spatial mapping of IHC, afferent and efferent synaptic markers, whose heterogeneity is putatively responsible for the diverse firing responses of the SGNs. As previously suggested, it will be critical that future studies addressing the mature cellular mechanisms of wide dynamic range sound coding in mice are performed only from 3 weeks of age, when a mature ribbon morphology (Sobkowicz et al., 1982; Wong et al., 2014) and spatial connectivity at IHCs (Liberman and Liberman, 2016) are established. Additionally, for a proper analysis of the modiolar-pillar gradient of the synapse properties, the IHCs would better be reconstructed individually as cylindrical models, to account for cell displacements likely related to ex vivo tissue manipulation (see Ohn et al., 2016). Moreover, current data from the literature are mixed from mice, cats and guinea pigs; therefor it will be significant to perform an exhaustive set of experiments in one species rather than assuming analogy. Indeed, once the spatial heterogeneity of synapse properties and associated postsynaptic firing is understood in mice, this animal model would be the best suited for genetic manipulation to investigate various candidate mechanisms, but potential differences between different mouse background and housing must be considered.

It will be essential to perform a complete ultrastructural study in order to assess the spatial gradient for the size and number of the synaptic ribbons, PDs and their associated SVs as well as PSDs along the modiolar/pillar axis. However, these studies would need to make the challenging effort to establish a high number of reconstructed cells from several animals. Additionally, completing comprehensive confocal/STED immunofluorescence microscopy studies analyzing the size and spatial mapping of multiple pre/postsynaptic as well as efferent markers would be valuable. In terms of physiology, STED live $\mathrm{Ca}^{2+}$ imaging under physiological buffering (Neef et al., 2018) will permit to obtain a more precise estimate of the spatial confinement of the $\mathrm{Ca}^{2+}$ influx at modiolar and pillar side $\mathrm{AZs}$ as compared to the confocal $\mathrm{Ca}^{2+}$ imaging performed here. Next, to characterize the distinct spatial connectivity among SGNs exhibiting different spiking behavior and their associated IHC presynapses, single unit recordings of SGNs with subsequent labelling and EM reconstruction of the afferent fibers towards the IHCs should be performed in the mouse as previously done in cats (Merchan-Perez and Liberman, 1996). Furthermore, 
experiments are required to directly study the relationship between presynaptic $\mathrm{Ca}^{2+}$ signal, exocytosis and postsynaptic response. Paired recording of IHCs and postsynaptic boutons (Goutman and Glowatzki, 2007) combined with simultaneous readout of the presynaptic $\mathrm{Ca}^{2+}$ hotspot would allow for establishing a more direct analysis of synaptic heterogeneity. Additionally, it could reveal different $\mathrm{Ca}^{2+}$ cooperativity of exocytosis and mode of release (MVR, UVR) between modiolar and pillar side AZs. Indeed inter-synaptic differences in the fashion of release could produce a broad spectrum of different EPSP amplitude and thus differentially regulate action potential generation in the respective postsynaptic SGN (Singer et al., 2009). In addition, iGluSnFr imaging (Marvin et al., 2013) would allow to simultaneously visualize the synaptic $\mathrm{Ca}^{2+}$ influx and the subsequent glutamate release. 


\section{REFERENCES}

Adamson, C.L., Reid, M.A., Mo, Z., Bowne-English, J., and Davis, R.L. (2002). Firing features and potassium channel content of murine spiral ganglion neurons vary with cochlear location. The Journal of Comparative Neurology 447, 331-350.

Assad, J.A., Shepherd, G.M.., and Corey, D.P. (1991). Tip-link integrity and mechanical transduction in vertebrate hair cells. Neuron 7, 985-994.

Augustine, G.J., Santamaria, F., and Tanaka, K. (2003). Local calcium signaling in neurons. Neuron 40, 331-346.

Bakondi, G., Pór, Á., Kovács, I., Szűcs, G., and Rusznák, Z. (2008). Voltage-gated K+ channel (Kv) subunit expression of the guinea pig spiral ganglion cells studied in a newly developed cochlear free-floating preparation. Brain Research 1210, 148-162.

Barbary, A.E. (1991). Auditory nerve of the normal and jaundiced rat. I. Spontaneous discharge rate and cochlear nerve histology. Hearing Research 54, 75-90.

Becker, L., Schnee, M.E., Niwa, M., Sun, W., Maxeiner, S., Talaei, S., Kachar, B., Rutherford, M.A., and Ricci, A.J. (2018). The presynaptic ribbon maintains vesicle populations at the hair cell afferent fiber synapse. ELife Sciences 7, e30241.

Bernstein, G.M., and Jones, O.T. (2007). Kinetics of internalization and degradation of N-type voltage-gated calcium channels: role of the alpha2/delta subunit. Cell Calcium 41, 27-40.

Beurg, M., Fettiplace, R., Nam, J.-H., and Ricci, A.J. (2009). Localization of inner hair cell mechanotransducer channels using high-speed calcium imaging. Nat. Neurosci. 12, 553-558.

Beurg, M., Michalski, N., Safieddine, S., Bouleau, Y., Schneggenburger, R., Chapman, E.R., Petit, C., and Dulon, D. (2010). Control of exocytosis by synaptotagmins and otoferlin in auditory hair cells. J. Neurosci 30, 13281-13290.

Beutner, D., and Moser, T. (2001). The presynaptic function of mouse cochlear inner hair cells during development of hearing. J Neurosci 21, 4593.

Bichet, D., Cornet, V., Geib, S., Carlier, E., Volsen, S., Hoshi, T., Mori, Y., and De Waard, M. (2000). The I-II loop of the Ca2+ channel [alpha] 1 subunit contains an endoplasmic reticulum retention signal antagonized by the [beta] subunit. Neuron 25, 177-190.

Black, J.L., 3rd (2003). The voltage-gated calcium channel gamma subunits: a review of the literature. J. Bioenerg. Biomembr 35, 649-660.

Bock, G., Gebhart, M., Scharinger, A., Jangsangthong, W., Busquet, P., Poggiani, C., Sartori, S., Mangoni, M.E., Sinnegger-Brauns, M.J., Herzig, S., et al. (2011). Functional Properties of a Newly Identified C-terminal Splice Variant of Cav1.3 L-type Ca2+ Channels. J. Biol. Chem. 286, 4273642748. 
Borst, J.G.G., and Sakmann, B. (1996). Calcium influx and transmitter release in a fast CNS synapse. Nature 383, 431-434.

Brandt, A., Striessnig, J., and Moser, T. (2003). CaV1. 3 channels are essential for development and presynaptic activity of cochlear inner hair cells. J Neurosci 23, 10832-10840.

Brandt, A., Khimich, D., and Moser, T. (2005). Few CaV1. 3 channels regulate the exocytosis of a synaptic vesicle at the hair cell ribbon synapse. J Neurosci 25, 11577.

Brehm, P., and Eckert, R. (1978). Calcium entry leads to inactivation of calcium channel in Paramecium. Science 202, 1203-1206.

Buraei, Z., and Yang, J. (2010). The ß subunit of voltage-gated Ca2+ channels. Physiol. Rev. 90, 1461-1506.

Buran, B.N., Strenzke, N., Neef, A., Gundelfinger, E.D., Moser, T., and Liberman, M.C. (2010). Onset coding is degraded in auditory nerve fibers from mutant mice lacking synaptic ribbons. J. Neurosci. 30, 7587-7597.

Carbone, E., and Lux, H.D. (1984). A low voltage-activated, fully inactivating Ca channel in vertebrate sensory neurones. Nature 310, 501-502.

Catterall, W.A., Perez-Reyes, E., Snutch, T.P., and Striessnig, J. (2005). International Union of Pharmacology. XLVIII. Nomenclature and Structure-Function Relationships of Voltage-Gated Calcium Channels. Pharmacol Rev 57, 411-425.

Cens, T., Rousset, M., Leyris, J.P., Fesquet, P., and Charnet, P. (2006). Voltage- and calciumdependent inactivation in high voltage-gated $\mathrm{Ca}(2+)$ channels. Prog Biophys Mol Biol 90, 104117.

Chan, D.K., and Hudspeth, A.J. (2005). Ca2+ current-driven nonlinear amplification by the mammalian cochlea in vitro. Nat Neurosci 8, 149-155.

Chapochnikov, N.M., Takago, H., Huang, C.-H., Pangršič, T., Khimich, D., Neef, J., Auge, E., Göttfert, F., Hell, S.W., Wichmann, C., et al. (2014). Uniquantal Release through a Dynamic Fusion Pore Is a Candidate Mechanism of Hair Cell Exocytosis. Neuron 17, 1389-1403.

Chen, W.C., Xue, H.Z., Hsu, Y. (Lucy), Liu, Q., Patel, S., and Davis, R.L. (2011). Complex distribution patterns of voltage-gated calcium channel $\alpha$-subunits in the spiral ganglion. Hearing Research 278, 52-68.

Corey, D.P., and Hudspeth, A.J. (1979). Ionic basis of the receptor potential in a vertebrate hair cell. Nature $281,675-677$.

Cui, G., Meyer, A.C., Calin-Jageman, I., Neef, J., Haeseleer, F., Moser, T., and Lee, A. (2007). Ca2+binding proteins tune Ca2+-feedback to Cav1. 3 channels in mouse auditory hair cells. The Journal of Physiology 585, 791-803.

Dafi, O., Berrou, L., Dodier, Y., Raybaud, A., Sauvé, R., and Parent, L. (2004). Negatively charged 
residues in the $\mathrm{N}$-terminal of the AID helix confer slow voltage dependent inactivation gating to CaV1.2. Biophys. J. 87, 3181-3192.

Davies, A., Kadurin, I., Alvarez-Laviada, A., Douglas, L., Nieto-Rostro, M., Bauer, C.S., Pratt, W.S., and Dolphin, A.C. (2010). The $\alpha 2 \delta$ subunits of voltage-gated calcium channels form GPI-anchored proteins, a posttranslational modification essential for function. PNAS 107, 1654-1659.

Davis, R.L., and Crozier, R.A. (2016). The Electrophysiological Signature of Spiral Ganglion Neurons. In The Primary Auditory Neurons of the Mammalian Cochlea, A. Dabdoub, B. Fritzsch, A.N. Popper, and R.R. Fay, eds. (New York, NY: Springer New York), pp. 85-116.

Davydova, D., Marini, C., King, C., Klueva, J., Bischof, F., Romorini, S., Montenegro-Venegas, C., Heine, M., Schneider, R., Schröder, M.S., et al. (2014). Bassoon specifically controls presynaptic P/Q-type $\mathrm{Ca}(2+)$ channels via RIM-binding protein. Neuron 82, 181-194.

DeMaria, C.D., Soong, T.W., Alseikhan, B.A., Alvania, R.S., and Yue, D.T. (2001). Calmodulin bifurcates the local Ca2+ signal that modulates P/Q-type Ca2+ channels. Nature 411, 484-489.

Dick, O., tom Dieck, S., Altrock, W.D., Ammermüller, J., Weiler, R., Garner, C.C., Gundelfinger, E.D., and Brandstätter, J.H. (2003). The Presynaptic Active Zone Protein Bassoon Is Essential for Photoreceptor Ribbon Synapse Formation in the Retina. Neuron 37, 775-786.

tom Dieck, S., Specht, D., Strenzke, N., Hida, Y., Krishnamoorthy, V., Schmidt, K.-F., Inoue, E., Ishizaki, H., Tanaka-Okamoto, M., Miyoshi, J., et al. (2012). Deletion of the presynaptic scaffold CAST reduces active zone size in rod photoreceptors and impairs visual processing. J Neurosci $32,12192-12203$.

Dolphin, A.C. (2013). The $\alpha 2 \delta$ subunits of voltage-gated calcium channels. Biochimica et Biophysica Acta (BBA) - Biomembranes 1828, 1541-1549.

Dou, H., Vazquez, A.E., Namkung, Y., Chu, H., Cardell, E.L., Nie, L., Parson, S., Shin, H.-S., and Yamoah, E.N. (2004). Null mutation of alpha1D Ca2+ channel gene results in deafness but no vestibular defect in mice. J Assoc Res Otolaryngol 5, 215-226.

Dulon, D., Papal, S., Patni, P., Cortese, M., Vincent, P.F.Y., Tertrais, M., Emptoz, A., Tlili, A., Bouleau, Y., Michel, V., et al. (2018). Clarin-1 gene transfer rescues auditory synaptopathy in model of Usher syndrome. J Clin Invest 128, 3382-3401.

Edmonds, B., Reyes, R., Schwaller, B., and Roberts, W.M. (2000). Calretinin modifies presynaptic calcium signaling in frog saccular hair cells. Nat Neurosci 3, 786-790.

Erickson, M.G., Alseikhan, B.A., Peterson, B.Z., and Yue, D.T. (2001). Preassociation of Calmodulin with Voltage-Gated Ca2+ Channels Revealed by FRET in Single Living Cells. Neuron 31, 973-985.

Ertel, E.A., Campbell, K.P., Harpold, M.M., Hofmann, F., Mori, Y., Perez-Reyes, E., Schwartz, A., Snutch, T.P., Tanabe, T., Birnbaumer, L., et al. (2000). Nomenclature of voltage-gated calcium channels. Neuron 25, 533-535.

Eybalin, M. (1993). Neurotransmitters and neuromodulators of the mammalian cochlea. Physiol. 
Rev 73, 309-373.

Eybalin, M., Charachon, G., and Renard, N. (1993). Dopaminergic lateral efferent innervation of the guinea-pig cochlea: Immunoelectron microscopy of catecholamine-synthesizing enzymes and effect of 6-hydroxydopamine. Neuroscience 54, 133-142.

Felix, D., and Ehrenberger, K. (1992). The efferent modulation of mammalian inner hair cell afferents. Hearing Research 64, 1-5.

Fell, B., Eckrich, S., Blum, K., Eckrich, T., Hecker, D., Obermair, G.J., Münkner, S., Flockerzi, V., Schick, B., and Engel, J. (2016). $\alpha 2 \delta 2$ Controls the Function and Trans-Synaptic Coupling of Cav1.3 Channels in Mouse Inner Hair Cells and Is Essential for Normal Hearing. J. Neurosci. 36, 1102411036.

Frank, T., Khimich, D., Neef, A., and Moser, T. (2009). Mechanisms contributing to synaptic Ca2+ signals and their heterogeneity in hair cells. Proc Natl Acad Sci U S A 106, 4483.

Frank, T., Rutherford, M.A., Strenzke, N., Neef, A., Pangršič, T., Khimich, D., Fejtova, A., Gundelfinger, E.D., Liberman, M.C., Harke, B., et al. (2010). Bassoon and the synaptic ribbon organize $\mathrm{Ca}^{2}+$ channels and vesicles to add release sites and promote refilling. Neuron $68,724-$ 738.

Fuchs, P.A., Glowatzki, E., and Moser, T. (2003). The afferent synapse of cochlear hair cells. Current Opinion in Neurobiology 13, 452-458.

Gao, B., Sekido, Y., Maximov, A., Saad, M., Forgacs, E., Latif, F., Wei, M.H., Lerman, M., Lee, J.H., Perez-Reyes, E., et al. (2000). Functional properties of a new voltage-dependent calcium channel alpha(2)delta auxiliary subunit gene (CACNA2D2). J. Biol. Chem. 275, 12237-12242.

Gebhart, M., Juhasz-Vedres, G., Zuccotti, A., Brandt, N., Engel, J., Trockenbacher, A., Kaur, G., Obermair, G.J., Knipper, M., Koschak, A., et al. (2010). Modulation of Cav1.3 Ca2+ channel gating by Rab3 interacting molecule. Mol. Cell. Neurosci. 44, 246-259.

Glauner, K.S., Mannuzzu, L.M., Gandhi, C.S., and Isacoff, E.Y. (1999). Spectroscopic mapping of voltage sensor movement in the Shaker potassium channel. Nature 402, 813-817.

Glowatzki, E., and Fuchs, P.A. (2000). Cholinergic synaptic inhibition of inner hair cells in the neonatal mammalian cochlea. Science 288, 2366.

Goutman, J.D., and Glowatzki, E. (2007). Time course and calcium dependence of transmitter release at a single ribbon synapse. Proc Natl Acad Sci U S A 104, 16341-16346.

Grabner, C.P., and Moser, T. (2018). Individual synaptic vesicles mediate stimulated exocytosis from cochlear inner hair cells. PNAS 115, 12811-12816.

Graydon, C.W., Zhang, J., Oesch, N.W., Sousa, A.A., Leapman, R.D., and Diamond, J.S. (2014). Passive Diffusion as a Mechanism Underlying Ribbon Synapse Vesicle Release and Resupply. J. Neurosci. 34, 8948-8962. 
Green, P.J., Warre, R., Hayes, P.D., McNaughton, N.C., Medhurst, A.D., Pangalos, M., Duckworth, D.M., and Randall, A.D. (2001). Kinetic modification of the alpha(1I) subunit-mediated T-type $\mathrm{Ca}(2+)$ channel by a human neuronal $\mathrm{Ca}(2+)$ channel gamma subunit. J. Physiol. (Lond.) $533,467-$ 478.

Groff, J.A., and Liberman, M.C. (2003). Modulation of cochlear afferent response by the lateral olivocochlear system: activation via electrical stimulation of the inferior colliculus. J. Neurophysiol. 90, 3178-3200.

Hackney, C.M., Mahendrasingam, S., Penn, A., and Fettiplace, R. (2005). The concentrations of calcium buffering proteins in mammalian cochlear hair cells. J Neurosci 25, 7867.

Haeseleer, F., Sokal, I., Verlinde, C.L., Erdjument-Bromage, H., Tempst, P., Pronin, A.N., Benovic, J.L., Fariss, R.N., and Palczewski, K. (2000). Five members of a novel $\mathrm{Ca}(2+)$-binding protein (CABP) subfamily with similarity to calmodulin. J. Biol. Chem 275, 1247-1260.

Haeseleer, F., Imanishi, Y., Maeda, T., Possin, D.E., Maeda, A., Lee, A., Rieke, F., and Palczewski, K. (2004). Essential role of Ca2+-binding protein 4, a Cav1.4 channel regulator, in photoreceptor synaptic function. Nat. Neurosci 7, 1079-1087.

Hagiwara, A., Kitahara, Y., Grabner, C.P., Vogl, C., Abe, M., Kitta, R., Ohta, K., Nakamura, K., Sakimura, K., Moser, T., et al. (2018). Cytomatrix proteins CAST and ELKS regulate retinal photoreceptor development and maintenance. The Journal of Cell Biology 217, 3993-4006.

Han, Y., Babai, N., Kaeser, P., Südhof, T.C., and Schneggenburger, R. (2015). RIM1 and RIM2 redundantly determine $\mathrm{Ca} 2+$ channel density and readily releasable pool size at a large hindbrain synapse. J. Neurophysiol. 113, 255-263.

Hanlon, M.R., Berrow, N.S., Dolphin, A.C., and Wallace, B.A. (1999). Modelling of a voltagedependent $\mathrm{Ca} 2+$ channel $b$ subunit as a basis for understanding its functional properties. Febs Lett 445, 366-370.

Hibino, H., Pironkova, R., Onwumere, O., Vologodskaia, M., Hudspeth, A.J., and Lesage, F. (2002). RIM - binding proteins (RBPs) couple Rab3 - interacting molecules (RIMs) to voltage - gated Ca2+ channels. Neuron 34, 411-423.

Hoppa, M.B., Lana, B., Margas, W., Dolphin, A.C., and Ryan, T.A. (2012). $\alpha 2 \delta$ expression sets presynaptic calcium channel abundance and release probability. Nature 486, 122-125.

Howard, J., and Hudspeth, A.J. (1988). Compliance of the hair bundle associated with gating of mechanoelectrical transduction channels in the bullfrog's saccular hair cell. Neuron 1, 189-199.

Huang, C.-H., and Moser, T. (2018). Ca2+ Regulates the Kinetics of Synaptic Vesicle Fusion at the Afferent Inner Hair Cell Synapse. Front Cell Neurosci 12, 364.

Hudspeth, A.J. (2008). Making an Effort to Listen: Mechanical Amplification in the Ear. Neuron 59, 530-545.

Hull, C., Studholme, K., Yazulla, S., and von Gersdorff, H. (2006). Diurnal changes in exocytosis 
and the number of synaptic ribbons at active zones of an ON-type bipolar cell terminal. J. Neurophysiol 96, 2025-2033.

Jackman, S.L., Choi, S.-Y., Thoreson, W.B., Rabl, K., Bartoletti, T.M., and Kramer, R.H. (2009). Role of the synaptic ribbon in transmitting the cone light response. Nat Neurosci 12, 303.

Jaramillo, F., and Hudspeth, A.J. (1991). Localization of the hair cell's transduction channels at the hair bundle's top by iontophoretic application of a channel blocker. Neuron 7, 409-420.

Jay, S.D., Ellis, S.B., McCue, A.F., Williams, M.E., Vedvick, T.S., Harpold, M.M., and Campbell, K.P. (1990). Primary structure of the gamma subunit of the DHP-sensitive calcium channel from skeletal muscle. Science 248, 490-492.

Jean, P., Lopez de la Morena, D., Michanski, S., Jaime Tobón, L.M., Chakrabarti, R., Picher, M.M., Neef, J., Jung, S., Gültas, M., Maxeiner, S., et al. (2018). The synaptic ribbon is critical for sound encoding at high rates and with temporal precision. Elife 7.

Jing, Z., Rutherford, M.A., Takago, H., Frank, T., Fejtova, A., Khimich, D., Moser, T., and Strenzke, N. (2013). Disruption of the presynaptic cytomatrix protein bassoon degrades ribbon anchorage, multiquantal release, and sound encoding at the hair cell afferent synapse. J Neurosci 33, 44564467.

Johnson, S.L., Marcotti, W., and Kros, C.J. (2005). Increase in efficiency and reduction in Ca2+ dependence of exocytosis during development of mouse inner hair cells. J Physiol 563, 177-191.

Johnson, S.L., Franz, C., Kuhn, S., Furness, D.N., Ruttiger, L., Munkner, S., Rivolta, M.N., Seward, E.P., Herschman, H.R., Engel, J., et al. (2010). Synaptotagmin IV determines the linear Ca2+ dependence of vesicle fusion at auditory ribbon synapses. Nat Neurosci 13, 45-52.

Jones, L.P., Wei, S., and Yue, D.T. (1998). Mechanism of Auxiliary Subunit Modulation of Neuronal a1E Calcium Channels. J Gen Physiol 112, 125-143.

Jung, S., Oshima-Takago, T., Chakrabarti, R., Wong, A.B., Jing, Z., Yamanbaeva, G., Picher, M.M., Wojcik, S.M., Göttfert, F., Predoehl, F., et al. (2015). Rab3-interacting molecules $2 \alpha$ and $2 \beta$ promote the abundance of voltage-gated CaV1.3 Ca2+ channels at hair cell active zones. Proc. Natl. Acad. Sci. U.S.A. 112, E3141-E3149.

Kaeser, P.S., Deng, L., Wang, Y., Dulubova, I., Liu, X., Rizo, J., and Südhof, T.C. (2011). RIM proteins tether $\mathrm{Ca} 2+$ channels to presynaptic active zones via a direct PDZ-domain interaction. Cell 144, 282-295.

Kandel, E.R., Schwartz, J.H., and Jessell, T. (2012). Principles of neural science (New York: McGraw-Hill Medical).

Kantardzhieva, A., Liberman, M.C., and Sewell, W.F. (2013). Quantitative analysis of ribbons, vesicles, and cisterns at the cat inner hair cell synapse: correlations with spontaneous rate. J. Comp. Neurol. 521, 3260-3271.

Katz, E., Elgoyhen, A.B., Gómez-Casati, M.E., Knipper, M., Vetter, D.E., Fuchs, P.A., and Glowatzki, 
E. (2004). Developmental regulation of nicotinic synapses on cochlear inner hair cells. J. Neurosci 24, 7814-7820.

Keen, E.C., and Hudspeth, A.J. (2006). Transfer characteristics of the hair cell's afferent synapse. Proc Natl Acad Sci U S A 103, 5537-5542.

Kennedy, H.J. (2002). Intracellular calcium regulation in inner hair cells from neonatal mice. Cell Calcium 31, 127-136.

Kennedy, H.J., Crawford, A.C., and Fettiplace, R. (2005). Force generation by mammalian hair bundles supports a role in cochlear amplification. Nature $433,880-883$.

Khimich, D., Nouvian, R., Pujol, R., tom Dieck, S., Egner, A., Gundelfinger, E.D., and Moser, T. (2005). Hair cell synaptic ribbons are essential for synchronous auditory signalling. Nature 434, 889-894.

Kiang, N.Y., Rho, J.M., Northrop, C.C., Liberman, M.C., and Ryugo, D.K. (1982). Hair-cell innervation by spiral ganglion cells in adult cats. Science $217,175-177$.

Kintscher, M., Wozny, C., Johenning, F.W., Schmitz, D., and Breustedt, J. (2013). Role of RIM1 $\alpha$ in short- and long-term synaptic plasticity at cerebellar parallel fibres. Nat Commun 4, 2392.

Kiyonaka, S., Wakamori, M., Miki, T., Uriu, Y., Nonaka, M., Bito, H., Beedle, A.M., Mori, E., Hara, Y., De Waard, M., et al. (2007). RIM1 confers sustained activity and neurotransmitter vesicle anchoring to presynaptic Ca2+ channels. Nat. Neurosci. 10, 691-701.

Krinner, S., Butola, T., Jung, S., Wichmann, C., and Moser, T. (2017). RIM-binding protein 2 promotes a large number of CaV1.3 Ca2+-channels and contributes to fast synaptic vesicle replenishment at hair cell active zones. Front Cell Neurosci.

Kuhn, S., Knirsch, M., Rüttiger, L., Kasperek, S., Winter, H., Freichel, M., Flockerzi, V., Knipper, M., and Engel, J. (2009). Ba2+ currents in inner and outer hair cells of mice lacking the voltagedependent Ca2+ channel subunits beta3 or beta4. Channels (Austin) 3, 366-376.

Legan, P.K., Lukashkina, V.A., Goodyear, R.J., Kössl, M., Russell, I.J., and Richardson, G.P. (2000). A Targeted Deletion in $\alpha$-Tectorin Reveals that the Tectorial Membrane Is Required for the Gain and Timing of Cochlear Feedback. Neuron 28, 273-285.

Lenzi, D., and von Gersdorff, H. (2001). Structure suggests function: the case for synaptic ribbons as exocytotic nanomachines. Bioessays $23,831-840$.

Liberman, M. (1982). Single-neuron labeling in the cat auditory nerve. Science 216, 1239-1241.

Liberman, M.C. (1978). Auditory-nerve response from cats raised in a low-noise chamber. J. Acoust. Soc. Am 63, 442-455.

Liberman, M.C. (1980). Morphological differences among radial afferent fibers in the cat cochlea: an electron-microscopic study of serial sections. Hear. Res. 3, 45-63. 
Liberman, M.C. (1990). Effects of chronic cochlear de-efferentation on auditory-nerve response. Hear. Res 49, 209-223.

Liberman, L.D., and Liberman, M.C. (2016). Postnatal maturation of auditory-nerve heterogeneity, as seen in spatial gradients of synapse morphology in the inner hair cell area. Hearing Research 339, 12-22.

Liberman, L.D., Wang, H., and Liberman, M.C. (2011). Opposing Gradients of Ribbon Size and AMPA Receptor Expression Underlie Sensitivity Differences among Cochlear-Nerve/Hair-Cell Synapses. J Neurosci 31, 801-808.

Liberman, M.C., Dodds, L.W., and Pierce, S. (1990). Afferent and efferent innervation of the cat cochlea: quantitative analysis with light and electron microscopy. J. Comp. Neurol 301, 443-460.

Liberman, M.C., Gao, J., He, D.Z.Z., Wu, X., Jia, S., and Zuo, J. (2002). Prestin is required for electromotility of the outer hair cell and for the cochlear amplifier. Nature 419, 300-304.

Liu, H., De Waard, M., Scott, V.E., Gurnett, C.A., Lennon, V.A., and Campbell, K.P. (1996). Identification of three subunits of the high affinity omega-conotoxin MVIIC-sensitive Ca2+ channel. J. Biol. Chem 271, 13804-13810.

Liu, Q., Lee, E., and Davis, R.L. (2014). Heterogeneous intrinsic excitability of murine spiral ganglion neurons is determined by Kv1 and HCN channels. Neuroscience 257, 96-110.

Llinás, R., Sugimori, M., Lin, J.W., and Cherksey, B. (1989). Blocking and isolation of a calcium channel from neurons in mammals and cephalopods utilizing a toxin fraction (FTX) from funnelweb spider poison. Proc. Natl. Acad. Sci. U.S.A 86, 1689-1693.

Logothetis, D.E., Movahedi, S., Satler, C., Lindpaintner, K., and Nadal-Ginard, B. (1992). Incremental reductions of positive charge within the S4 region of a voltage-gated $\mathrm{K}+$ channel result in corresponding decreases in gating charge. Neuron $8,531-540$.

Lohi, H., Kujala, M., Kerkelä, E., Saarialho-Kere, U., Kestilä, M., and Kere, J. (2000). Mapping of five new putative anion transporter genes in human and characterization of SLC26A6, a candidate gene for pancreatic anion exchanger. Genomics 70, 102-112.

Lukacs, G.L. (2016). Proteostasis: Chaperoning for hearing loss. Nature Chemical Biology 12, 388389.

Lumpkin, E.A., and Hudspeth, A.J. (1995). Detection of Ca2+ entry through mechanosensitive channels localizes the site of mechanoelectrical transduction in hair cells. Proceedings of the National Academy of Sciences 92, 10297.

Lv, C., Stewart, W.J., Akanyeti, O., Frederick, C., Zhu, J., Santos-Sacchi, J., Sheets, L., Liao, J.C., and Zenisek, D. (2016). Synaptic Ribbons Require Ribeye for Electron Density, Proper Synaptic Localization, and Recruitment of Calcium Channels. Cell Reports 15, 2784-2795.

Maison, S.F., Adams, J.C., and Liberman, M.C. (2003). Olivocochlear innervation in the mouse: immunocytochemical maps, crossed versus uncrossed contributions, and transmitter 
colocalization. J. Comp. Neurol 455, 406-416.

Maison, S.F., Liu, X.-P., Eatock, R.A., Sibley, D.R., Grandy, D.K., and Liberman, M.C. (2012). Dopaminergic Signaling in the Cochlea: Receptor Expression Patterns and Deletion Phenotypes. J. Neurosci. 32, 344-355.

Marvin, J.S., Borghuis, B.G., Tian, L., Cichon, J., Harnett, M.T., Akerboom, J., Gordus, A., Renninger, S.L., Chen, T.-W., Bargmann, C.I., et al. (2013). An optimized fluorescent probe for visualizing glutamate neurotransmission. Nat Meth 10, 162-170.

Matveev, V., Bertram, R., and Sherman, A. (2009). Ca2+ Current versus Ca2+ Channel Cooperativity of Exocytosis. J Neurosci 29, 12196-12209.

Maxeiner, S., Luo, F., Tan, A., Schmitz, F., and Südhof, T.C. (2016). How to make a synaptic ribbon: RIBEYE deletion abolishes ribbons in retinal synapses and disrupts neurotransmitter release. The EMBO Journal 35, 1098-1114.

Mehta, B., Snellman, J., Chen, S., Li, W., and Zenisek, D. (2013). Synaptic Ribbons Influence the Size and Frequency of Miniature-like Evoked Postsynaptic Currents. Neuron 77, 516-527.

Meir, A., and Dolphin, A.C. (2002). Kinetics and Gbetagamma modulation of Ca(v)2.2 channels with different auxiliary beta subunits. Pflugers Arch. 444, 263-275.

Merchan-Perez, A., and Liberman, M.C. (1996). Ultrastructural differences among afferent synapses on cochlear hair cells: correlations with spontaneous discharge rate. J. Comp. Neurol $371,208-221$.

Meyer, A.C., Frank, T., Khimich, D., Hoch, G., Riedel, D., Chapochnikov, N.M., Yarin, Y.M., Harke, B., Hell, S.W., Egner, A., et al. (2009). Tuning of synapse number, structure and function in the cochlea. Nat Neurosci 12, 444-453.

Müller, U. (2008). Cadherins and mechanotransduction by hair cells. Current Opinion in Cell Biology 20, 557-566.

Muresan, V., Lyass, A., and Schnapp, B.J. (1999). The Kinesin Motor KIF3A Is a Component of the Presynaptic Ribbon in Vertebrate Photoreceptors. J. Neurosci. 19, 1027-1037.

Nadol, J.B. (1988). Comparative anatomy of the cochlea and auditory nerve in mammals. Hearing Research 34, 253-266.

Naraghi, M. (1997). T-jump study of calcium binding kinetics of calcium chelators. Cell Calcium 22, 255-268.

Neef, J., Gehrt, A., Bulankina, A.V., Meyer, A.C., Riedel, D., Gregg, R.G., Strenzke, N., and Moser, T. (2009). The Ca2+ Channel Subunit beta2 Regulates Ca2+ Channel Abundance and Function in Inner Hair Cells and Is Required for Hearing. J Neurosci 29, 10730.

Neef, J., Urban, N.T., Ohn, T.-L., Frank, T., Jean, P., Hell, S.W., Willig, K.I., and Moser, T. (2018). Quantitative optical nanophysiology of $\mathrm{Ca} 2+$ signaling at inner hair cell active zones. Nature 
Communications 9, 290.

Neely, A., Wei, X., Olcese, R., Birnbaumer, L., and Stefani, E. (1993). Potentiation by the beta subunit of the ratio of the ionic current to the charge movement in the cardiac calcium channel. Science 262, 575.

Neher, E. (1998). Vesicle pools and Ca2+ microdomains: new tools for understanding their roles in neurotransmitter release. Neuron 20, 389-399.

Neher, E., and Augustine, G.J. (1992). Calcium gradients and buffers in bovine chromaffin cells. J. Physiol. (Lond.) 450, 273-301.

Nicoll, R.A., Tomita, S., and Bredt, D.S. (2006). Auxiliary Subunits Assist AMPA-Type Glutamate Receptors. Science 311, 1253-1256.

Niidome, T., Kim, M.-S., Friedrich, T., and Mori, Y. (1992). Molecular cloning and characterization of a novel calcium channel from rabbit brain. FEBS Letters 308, 7-13.

Nilius, B., Hess, P., Lansman, J.B., and Tsien, R.W. (1985). A novel type of cardiac calcium channel in ventricular cells. Nature $316,443-446$.

Nouvian, R., Neef, J., Bulankina, A.V., Reisinger, E., Pangršič, T., Frank, T., Sikorra, S., Brose, N., Binz, T., and Moser, T. (2011). Exocytosis at the hair cell ribbon synapse apparently operates without neuronal SNARE proteins. Nat Neurosci 14, 411-413.

Nowycky, M.C., Fox, A.P., and Tsien, R.W. (1985). Three types of neuronal calcium channel with different calcium agonist sensitivity. Nature 316, 440-443.

Ohlemiller, K.K., and Echteler, S.M. (1990). Functional correlates of characteristic frequency in single cochlear nerve fibers of the Mongolian gerbil. J. Comp. Physiol. A 167, 329-338.

Ohn, T.-L., Rutherford, M.A., Jing, Z., Jung, S., Duque-Afonso, C.J., Hoch, G., Picher, M.M., Scharinger, A., Strenzke, N., and Moser, T. (2016). Hair cells use active zones with different voltage dependence of $\mathrm{Ca} 2+$ influx to decompose sounds into complementary neural codes. PNAS 113, 201605737.

Oliver, D., He, D.Z.Z., Klöcker, N., Ludwig, J., Schulte, U., Waldegger, S., Ruppersberg, J.P., Dallos, P., and Fakler, B. (2001). Intracellular Anions as the Voltage Sensor of Prestin, the Outer Hair Cell Motor Protein. Science 292, 2340-2343.

Osten, P., and Sternbach, Y. (2006). Learning from stargazin: the mouse, the phenotype and the unexpected. Current Opinion in Neurobiology 16, 275-280.

Oz, S., Tsemakhovich, V., Christel, C.J., Lee, A., and Dascal, N. (2011). CaBP1 Regulates Voltagedependent Inactivation and Activation of CaV1.2 (L-type) Calcium Channels. J. Biol. Chem. 286, 13945-13953.

Pangrsic, T., Gabrielaitis, M., Michanski, S., Schwaller, B., Wolf, F., Strenzke, N., and Moser, T. (2015). EF-hand protein Ca2+ buffers regulate Ca2+ influx and exocytosis in sensory hair cells. $\mathrm{P}$ 
Natl Acad Sci USA 112, E1028-E1037.

Peterson, B.Z., DeMaria, C.D., Adelman, J.P., and Yue, D.T. (1999). Calmodulin is the Ca2+ sensor for Ca2+-dependent inactivation of L-type calcium channels. Neuron 22, 549-558.

Petitpré, C., Wu, H., Sharma, A., Tokarska, A., Fontanet, P., Wang, Y., Helmbacher, F., Yackle, K., Silberberg, G., Hadjab, S., et al. (2018). Neuronal heterogeneity and stereotyped connectivity in the auditory afferent system. Nature Communications 9, 3691.

Picher, M.M., Oprişoreanu, A.-M., Jung, S., Michel, K., Schoch, S., and Moser, T. (2017a). Rab Interacting Molecules 2 and 3 Directly Interact with the Pore-Forming CaV1.3 Ca2+ Channel Subunit and Promote Its Membrane Expression. Front Cell Neurosci 11.

Picher, M.M., Gehrt, A., Meese, S., Ivanovic, A., Predoehl, F., Jung, S., Schrauwen, I., Dragonetti, A.G., Colombo, R., Camp, G.V., et al. (2017b). Ca2+-binding protein 2 inhibits Ca2+-channel inactivation in mouse inner hair cells. PNAS 114, E1717-E1726.

Pickles, J.O., Comis, S.D., and Osborne, M.P. (1984). Cross-links between stereocilia in the guinea pig organ of Corti, and their possible relation to sensory transduction. Hearing Research 15, 103112.

Pitt, G.S., Zühlke, R.D., Hudmon, A., Schulman, H., Reuter, H., and Tsien, R.W. (2001). Molecular basis of calmodulin tethering and Ca2+-dependent inactivation of L-type Ca2+ channels. J. Biol. Chem. 276, 30794-30802.

Platzer, J., Engel, J., Schrott-Fischer, A., Stephan, K., Bova, S., Chen, H., Zheng, H., and Striessnig, J. (2000). Congenital deafness and sinoatrial node dysfunction in mice lacking class D L-type Ca2+ channels. Cell 102, 89-97.

Pragnell, M., De Waard, M., Mori, Y., Tanabe, T., Snutch, T.P., and Campbell, K.P. (1994). Calcium channel beta-subunit binds to a conserved motif in the I-II cytoplasmic linker of the alpha 1subunit. Nature 368, 67-70.

Qin, N., Olcese, R., Bransby, M., Lin, T., and Birnbaumer, L. (1999). Ca2+-induced inhibition of the cardiac Ca2+ channel depends on calmodulin. Proc. Natl. Acad. Sci. U.S.A. 96, 2435-2438.

Raphael, Y., and Altschuler, R.A. (2003). Structure and innervation of the cochlea. Brain Research Bulletin 60, 397-422.

Regus-Leidig, H., Ott, C., Löhner, M., Atorf, J., Fuchs, M., Sedmak, T., Kremers, J., Fejtová, A., Gundelfinger, E.D., and Brandstätter, J.H. (2013). Identification and Immunocytochemical Characterization of Piccolino, a Novel Piccolo Splice Variant Selectively Expressed at Sensory Ribbon Synapses of the Eye and Ear. PLoS ONE 8, e70373.

Regus-Leidig, H., Fuchs, M., Löhner, M., Leist, S.R., Leal-Ortiz, S., Chiodo, V.A., Hauswirth, W.W., Garner, C.C., and Brandstätter, J.H. (2014). In vivo knockdown of Piccolino disrupts presynaptic ribbon morphology in mouse photoreceptor synapses. Front Cell Neurosci 8, 259.

Reisinger, E., Bresee, C., Neef, J., Nair, R., Reuter, K., Bulankina, A., Nouvian, R., Koch, M., 
Bückers, J., Kastrup, L., et al. (2011). Probing the functional equivalence of otoferlin and synaptotagmin 1 in exocytosis. J. Neurosci. 31, 4886-4895.

Rieke, F., Lee, A., and Haeseleer, F. (2008). Characterization of Ca2+-binding protein 5 knockout mouse retina. Invest. Ophthalmol. Vis. Sci. 49, 5126-5135.

Roberts, W.M. (1993). Spatial calcium buffering in saccular hair cells. Nature 363, 74-76.

Roberts, W.M. (1994). Localization of calcium signals by a mobile calcium buffer in frog saccular hair cells. J. Neurosci 14, 3246-3262.

Roberts, W.M., Jacobs, R.A., and Hudspeth, A.J. (1990). Colocalization of ion channels involved in frequency selectivity and synaptic transmission at presynaptic active zones of hair cells. J Neurosci 10, 3664-3684.

Robertson, D., and Paki, B. (2002). Role of L-Type Ca2+ Channels in Transmitter Release From Mammalian Inner Hair Cells. II. Single-Neuron Activity. Journal of Neurophysiology 87, 27342740.

Ruel, J., Nouvian, R., D’Aldin, C.G., Pujol, R., Eybalin, M., and Puel, J.-L. (2001). Dopamine inhibition of auditory nerve activity in the adult mammalian cochlea. European Journal of Neuroscience 14, 977-986.

Ruel, J., Wang, J., Rebillard, G., Eybalin, M., Lloyd, R., Pujol, R., and Puel, J.-L. (2007). Physiology, pharmacology and plasticity at the inner hair cell synaptic complex. Hear. Res. 227, 19-27.

Ruel, J., Emery, S., Nouvian, R., Bersot, T., Amilhon, B., Van Rybroek, J.M., Rebillard, G., Lenoir, M., Eybalin, M., Delprat, B., et al. (2008). Impairment of SLC17A8 Encoding Vesicular Glutamate Transporter-3, VGLUT3, Underlies Nonsyndromic Deafness DFNA25 and Inner Hair Cell Dysfunction in Null Mice. Am J Hum Genet 83, 278-292.

Rutherford, M.A., and Moser, T. (2016). The Ribbon Synapse Between Type I Spiral Ganglion Neurons and Inner Hair Cells. In The Primary Auditory Neurons of the Mammalian Cochlea, A. Dabdoub, B. Fritzsch, A.N. Popper, and R.R. Fay, eds. (Springer New York), pp. 117-156.

Rutherford, M.A., Chapochnikov, N.M., and Moser, T. (2012). Spike Encoding of Neurotransmitter Release Timing by Spiral Ganglion Neurons of the Cochlea. J. Neurosci. 32, 4773-4789.

Safieddine, S., and Eybalin, M. (1992). Co-expression of NMDA and AMPA/kainate receptor mRNAs in cochlear neurones. Neuroreport 3, 1145-1148.

Safieddine, S., and Wenthold, R.J. (1999). SNARE complex at the ribbon synapses of cochlear hair cells: analysis of synaptic vesicle- and synaptic membrane-associated proteins. European Journal of Neuroscience 11, 803-812.

Schmitz, F., Königstorfer, A., and Südhof, T.C. (2000). RIBEYE, a component of synaptic ribbons: a protein's journey through evolution provides insight into synaptic ribbon function. Neuron 28 , 857-872. 
Schrauwen, I., Helfmann, S., Inagaki, A., Predoehl, F., Tabatabaiefar, M.A., Picher, M.M., Sommen, M., Seco, C.Z., Oostrik, J., Kremer, H., et al. (2012). A Mutation in CABP2, Expressed in Cochlear Hair Cells, Causes Autosomal-Recessive Hearing Impairment. Am J Hum Genet 91, 636645.

Seal, R.P., Akil, O., Yi, E., Weber, C.M., Grant, L., Yoo, J., Clause, A., Kandler, K., Noebels, J.L., Glowatzki, E., et al. (2008). Sensorineural Deafness and Seizures in Mice Lacking Vesicular Glutamate Transporter 3. Neuron 57, 263-275.

Sewell, W.F. (1984). The relation between the endocochlear potential and spontaneous activity in auditory nerve fibres of the cat. The Journal of Physiology 347, 685-696.

Sheets, L., Trapani, J.G., Mo, W., Obholzer, N., and Nicolson, T. (2011). Ribeye is required for presynaptic CaV1.3a channel localization and afferent innervation of sensory hair cells. Development 138, 1309-1319.

Sheets, L., He, X.J., Olt, J., Schreck, M., Petralia, R.S., Wang, Y.-X., Zhang, Q., Beirl, A., Nicolson, T., Marcotti, W., et al. (2017). Enlargement of Ribbons in Zebrafish Hair Cells Increases Calcium Currents But Disrupts Afferent Spontaneous Activity and Timing of Stimulus Onset. J. Neurosci. 37, 6299-6313.

Shen, Y., Yu, D., Hiel, H., Liao, P., Yue, D.T., Fuchs, P.A., and Soong, T.W. (2006). Alternative splicing of the $\mathrm{Ca}(\mathrm{v}) 1.3$ channel IQ domain, a molecular switch for Ca2+-dependent inactivation within auditory hair cells. J. Neurosci 26, 10690-10699.

Shrestha, B.R., Chia, C., Wu, L., Kujawa, S.G., Liberman, M.C., and Goodrich, L.V. (2018). Sensory Neuron Diversity in the Inner Ear Is Shaped by Activity. Cell 174, 1229-1246.e17.

Siemens, J., Lillo, C., Dumont, R.A., Reynolds, A., Williams, D.S., Gillespie, P.G., and Müller, U. (2004). Cadherin 23 is a component of the tip link in hair-cell stereocilia. Nature 428, 950-955.

Singer, J.H., Glowatzki, E., Moser, T., Strowbridge, B.W., Bhandawat, V., and Sampath, A.P. (2009). Functional properties of synaptic transmission in primary sense organs. J. Neurosci. 29, 12802-12806.

Singh, A., Gebhart, M., Fritsch, R., Sinnegger-Brauns, M.J., Poggiani, C., Hoda, J.-C., Engel, J., Romanin, C., Striessnig, J., and Koschak, A. (2008). Modulation of Voltage- and Ca2+-dependent Gating of CaV1.3 L-type Calcium Channels by Alternative Splicing of a C-terminal Regulatory Domain. J. Biol. Chem. 283, 20733-20744.

Sipe, C.W., and Lu, X. (2011). Kif3a regulates planar polarization of auditory hair cells through both ciliary and non-ciliary mechanisms. Development 138, 3441-3449.

Sobkowicz, H.M., and Emmerling, M.R. (1989). Development of acetylcholinesterase-positive neuronal pathways in the cochlea of the mouse. J. Neurocytol. 18, 209-224.

Sobkowicz, H.M., Rose, J.E., Scott, G.E., and Slapnick, S.M. (1982). Ribbon synapses in the developing intact and cultured organ of Corti in the mouse. J. Neurosci. 2, 942-957. 
Söllner, C., Rauch, G.-J., Siemens, J., Geisler, R., Schuster, S.C., Tübingen 2000 Screen Consortium, the, Müller, U., and Nicolson, T. (2004). Mutations in cadherin 23 affect tip links in zebrafish sensory hair cells. Nature 428, 955-959.

Spoendlin, H. (1969). Innervation patterns in the organ of corti of the cat. Acta Otolaryngol. 67, 239-254.

Spoendlin, H. (1972). Innervation densities of the cochlea. Acta Otolaryngol. 73, 235-248.

Sterling, P., and Matthews, G. (2005). Structure and function of ribbon synapses. Trends in Neurosciences 28, 20-29.

Stotz, S.C., and Zamponi, G.W. (2001). Identification of Inactivation Determinants in the Domain IIS6 Region of High Voltage-activated Calcium Channels. J. Biol. Chem. 276, 33001-33010.

Sun, S., Babola, T., Pregernig, G., So, K.S., Nguyen, M., Su, S.-S.M., Palermo, A.T., Bergles, D.E., Burns, J.C., and Müller, U. (2018). Hair Cell Mechanotransduction Regulates Spontaneous Activity and Spiral Ganglion Subtype Specification in the Auditory System. Cell 174, 12471263.e15.

Taberner, A.M., and Liberman, M.C. (2005). Response Properties of Single Auditory Nerve Fibers in the Mouse. J Neurophysiol 93, 557-569.

Tadross, M.R., Ben Johny, M., and Yue, D.T. (2010). Molecular endpoints of Ca2+/calmodulinand voltage-dependent inactivation of Ca(v)1.3 channels. J. Gen. Physiol 135, 197-215.

Tan, B.Z., Jiang, F., Tan, M.Y., Yu, D., Huang, H., Shen, Y., and Soong, T.W. (2011). Functional characterization of alternative splicing in the C-terminus of L-type CaV1.3 channels. Journal of Biological Chemistry.

Tarchini, B., Tadenev, A.L.D., Devanney, N., and Cayouette, M. (2016). A link between planar polarity and staircase-like bundle architecture in hair cells. Development 143, 3926-3932.

Tsien, R.W., Lipscombe, D., Madison, D., Bley, K., and Fox, A. (1995). Reflections on Ca(2+)channel diversity, 1988-1994. Trends Neurosci 18, 52-54.

Williams, M.E., Marubio, L.M., Deal, C.R., Hans, M., Brust, P.F., Philipson, L.H., Miller, R.J., Johnson, E.C., Harpold, M.M., and Ellis, S.B. (1994). Structure and functional characterization of neuronal alpha $1 \mathrm{E}$ calcium channel subtypes. J. Biol. Chem 269, 22347-22357.

Winter, I.M., Robertson, D., and Yates, G.K. (1990). Diversity of characteristic frequency rateintensity functions in guinea pig auditory nerve fibres. Hearing Research 45, 191-202.

Witcher, D.R., De Waard, M., Sakamoto, J., Franzini-Armstrong, C., Pragnell, M., Kahl, S.D., and Campbell, K.P. (1993). Subunit identification and reconstitution of the N-type Ca2+ channel complex purified from brain. Science $261,486-489$.

Wong, A.B., Rutherford, M.A., Gabrielaitis, M., Pangršič, T., Göttfert, F., Frank, T., Michanski, S., Hell, S., Wolf, F., Wichmann, C., et al. (2014). Developmental refinement of hair cell synapses 
tightens the coupling of Ca2+ influx to exocytosis. EMBO J 33, 247-264.

Yamada, W.M., and Zucker, R.S. (1992). Time course of transmitter release calculated from simulations of a calcium diffusion model. Biophysical Journal 61, 671-682.

Yang, P.S., Alseikhan, B.A., Hiel, H., Grant, L., Mori, M.X., Yang, W., Fuchs, P.A., and Yue, D.T. (2006). Switching of Ca2+-dependent inactivation of $\mathrm{Ca}(\mathrm{v}) 1.3$ channels by calcium binding proteins of auditory hair cells. J. Neurosci 26, 10677-10689.

Yin, Y., Liberman, L.D., Maison, S.F., and Liberman, M.C. (2014). Olivocochlear innervation maintains the normal modiolar-pillar and habenular-cuticular gradients in cochlear synaptic morphology. J. Assoc. Res. Otolaryngol. 15, 571-583.

Zampini, V., Johnson, S.L., Franz, C., Lawrence, N.D., Münkner, S., Engel, J., Knipper, M., Magistretti, J., Masetto, S., and Marcotti, W. (2010). Elementary properties of CaV1.3 Ca(2+) channels expressed in mouse cochlear inner hair cells. J Physiol 588, 187-199.

Zanazzi, G., and Matthews, G. (2009). The molecular architecture of ribbon presynaptic terminals. Mol. Neurobiol 39, 130-148.

Zeitz, C., Kloeckener-Gruissem, B., Forster, U., Kohl, S., Magyar, I., Wissinger, B., Mátyás, G., Borruat, F.-X., Schorderet, D.F., Zrenner, E., et al. (2006). Mutations in CABP4, the gene encoding the $\mathrm{Ca} 2+$-binding protein 4 , cause autosomal recessive night blindness. Am. J. Hum. Genet 79, 657-667.

Zenisek, D., Steyer, J.A., and Almers, W. (2000). Transport, capture and exocytosis of single synaptic vesicles at active zones. Nature $406,849-854$.

Zenisek, D., Davila, V., Wan, L., and Almers, W. (2003). Imaging calcium entry sites and ribbon structures in two presynaptic cells. J. Neurosci 23, 2538-2548.

Zenisek, D., Horst, N.K., Merrifield, C., Sterling, P., and Matthews, G. (2004). Visualizing synaptic ribbons in the living cell. J. Neurosci 24, 9752-9759.

Zhang, L., Engler, S., Koepcke, L., Steenken, F., and Köppl, C. (2018). Concurrent gradients of ribbon volume and AMPA-receptor patch volume in cochlear afferent synapses on gerbil inner hair cells. Hearing Research 364, 81-89.

Zheng, J., Shen, W., He, D.Z.., Long, K.B., Madison, L.D., and Dallos, P. (2000). Prestin is the motor protein of cochlear outer hair cells. Nature 405, 149-155.

Zhou, H., Kim, S.-A., Kirk, E.A., Tippens, A.L., Sun, H., Haeseleer, F., and Lee, A. (2004). Ca2+Binding Protein-1 Facilitates and Forms a Postsynaptic Complex with Cav1.2 (L-Type) Ca2+ Channels. J. Neurosci. 24, 4698-4708.

Zühlke, R.D., Pitt, G.S., Deisseroth, K., Tsien, R.W., and Reuter, H. (1999). Calmodulin supports both inactivation and facilitation of L-type calcium channels. Nature 399, 159-162. 
I would like to thank first Tobias Moser for accepting me in his laboratory and his great supervision. He provided me all the help and resources I needed these past four years and gave me the chance to be involved in various and high quality projects. I am also appreciative for his considerable support in building my post-doctoral scientific carrier. I thank Thomas Dresbach and Andreas Neef for their suggestions, help and supervision of the progress of the projects.

I am very grateful to all my collaborators through my different projects within and aside my thesis. I thank my lab partners involved in the great team work for the Ribeye project leading to my first peer-reviewed publication and made it a great experience. I am also thankful to Matthew Kelley and Hanna Sherrill for the Pou4F1 project, and Basile Tarchini for his great collaboration on the Gai project.

I would also like to thank Jakob Neef for always being present when I needed help and advice, Carlos Duque Afonso who introduced me to the setup, analysis and technique, and Tzu-Lun Ohn for his pioneer work. Many thanks to Susann Muller and Patricia Räke-Kügler for their help with every administrative procedures, to Gerhard Hoch for developing analysis tool, and Christiane Senger-Freitag and Sandra Gerke for their daily assistance in the lab and considerable genotyping work.

Last but not least, I thank the CNMPB and SFB project A2 for their financial support. 